
Fibrillar structures in mixed systems

Jinfeng Peng 


\section{Thesis committee}

\section{Promotor}

Prof. Dr E. van der Linden

Professor of Physics and Physical Chemistry of Foods

Wageningen University

\section{Co-promotors}

Dr P. Venema

Assistant Professor, Physics and Physical Chemistry of Foods

Wageningen University

Dr K.P. Velikov

Assistant Professor, Soft Condensed Matter

Utrecht University, The Netherlands

\section{Other members}

Prof. Dr J.P.M. van Duynhoven, Wageningen University

Prof. Dr J. van der Gucht, Wageningen University

Prof. Dr A.J. van der Goot, Wageningen University

Dr R.H. Tromp, Utrecht University, The Netherlands

This research was conducted under the auspices of the Graduate School VLAG (Advanced studies in Food Technology, Agrobiotechnology, Nutrition and Health Sciences). 


\title{
Fibrillar structures in mixed systems
}

\author{
Jinfeng Peng
}

\section{Thesis}

submitted in fulfilment of the requirements for the degree of doctor at Wageningen University

by the authority of the Rector Magnificus

$$
\text { Prof. Dr A.P.J. Mol }
$$

in the presence of the

Thesis Committee appointed by the Academic Board to be defended in public on Tuesday, $30^{\text {th }}$ August 2016 at 4 p.m. in the Aula. 
Jinfeng Peng

Fibrillar structures in mixed systems,

292 pages.

PhD thesis, Wageningen University, Wageningen, NL (2016)

With references, with summary in English

ISBN 978-94-6257-826-5

DOI 10.18174/383192 
To my parents 



\section{Table of contents}

Chapter 2 Rheology and microstructure of dispersions of whey proteins and cellulose microfibrils

Chapter 3 Rheology and microstructure of dispersions of protein fibrils and cellulose microfibrils

Chapter 4 Duplex gels from whey protein isolate and cellulose microfibrils

Chapter 5 Directed growth of protein fibrils in the presence of cellulose microfibrils

Chapter 6 Protein fibrils induce emulsion stabilization

Chapter 7 Stability of colloidal dispersions in the presence of protein fibrils

Chapter 8 Tuning emulsion stability using protein-cellulose bi-fibrillar networks

Chapter 9 General discussion

Summary

Acknowledgements

About the author

List of Publications 



\section{Chapter 1}

\section{General introduction}


Chapter 1 


\subsection{Introduction}

Foods often contain fibrillar structures, for example, in the form of polysaccharides like cellulose, xanthan and carrageenan, or in the form of proteins like collagen. Under appropriate conditions, proteins, such as whey proteins, ${ }^{1}$ egg white proteins, ${ }^{2,3}$ soy proteins, ${ }^{4}$ and pea proteins ${ }^{5}$ also have the ability to form fibrillar structures.

The main functionalities of fibrillar structures are thickening, gelling, and stabilizing. ${ }^{6}$ The key parameters to these functionalities of the fibrils are length distribution, aspect ratio (length divided by diameter) and flexibility. These parameters play a key role in the interactions between fibrils, and between fibrils and other structures. ${ }^{6}$

In this thesis, we focus on two types of fibrillar structures: cellulose microfibrils from bacterial cellulose and protein based fibrils from whey protein isolate (WPI). For successful application of fibrillar structures in foods, it is essential to understand their behaviour in more complex systems containing other components. We therefore investigate the behaviour of systems containing spherical and fibrillar structures.

\subsubsection{Whey proteins and fibrils}

As a by-product of cheese-making and casein manufacturing, whey proteins have received considerable attention in food industry due to its nutritional value and functional properties. $^{7}$ Whey protein isolate (WPI) is a mixture of proteins consisting of mainly $\beta$ lactoglobulin ( 60\%, $\beta$-lg), $\alpha$-lactalbumin and bovine serum albumin. ${ }^{8}$ The behaviour of WPI has been found to be mainly governed by $\beta$-lg. ${ }^{9}$ Various protein structures can be formed under different conditions, ranging from linear to spherical aggregates. The structures formed and their properties are strongly dependent on the protein concentration, heating temperature, $\mathrm{pH}$, ionic strength and the presence of other components etc.. This further leads to different functionalities of the proteins in foods. ${ }^{10-12}$ Upon heating the proteins at low ionic strength at neutral acidity $(\mathrm{pH} 7)$, where the $\mathrm{pH}$ is away from the iso-electric point ( $\mathrm{pl}$ ) of 5.3, fine stranded aggregates are formed due to the strong electrostatic repulsion between the proteins. ${ }^{13}$ Above the so-called gelling concentration of the protein, fine-stranded gels can be obtained. ${ }^{14}$ The gel structure can be tuned from fine-stranded to particulate by varying the ionic strength (i.e. by the 
addition of salt). ${ }^{15-17}$ Numerous studies have been performed on understanding the heatinduced denaturation and gelation of WPI at neutral $\mathrm{pH}$, and their properties and functionalities under different conditions. ${ }^{9,11,13-29}$

When WPI is heated at $\mathrm{pH} 2$ at low ionic strength for several hours, rod-like fibrils are formed (Figure 1). Under this condition, $\beta$-lg is found to be the only protein involved in fibril formation. ${ }^{30}$ The process of fibril formation consists of two steps; the protein first hydrolyses to peptides and afterwards, a part of the peptides assemble into fibrils. ${ }^{31}$ The resulted fibrils are semi-flexible and polydispersed in their length. ${ }^{32}$ Their length is in the order of micrometers, and their thickness around $4 \mathrm{~nm} \cdot{ }^{33-35}$ It has also been found that the thickness and persistence length of the fibrils are dependent on the number of strands composing the fibrils, and the number of strands varies under different conditions. ${ }^{36-38}$ The fibril properties depend on conditions, like protein concentration, heating time and temperature, $\mathrm{pH}$ and salt concentration. ${ }^{30,39-41}$

The physical properties of the fibrils and their gels have been studied extensively. ${ }^{34,42-48}$ Upon increasing fibril concentration, the fibril solution transits from the dilute regime, via the semi-dilute regime into the nematic regimes $(\geq 0.4 \mathrm{wt} \%){ }^{6,32,48,49}$ It was found that fibrils can increase the viscosity of a WPI solution, ${ }^{42}$ thereby acting as a thickening agent. Both cold-set ${ }^{50}$ and heat-induced fibril gels can be formed. ${ }^{17,26,34,41,43,44,46,51-54}$ The application of fibrils in micro-encapsulation has also been reported. ${ }^{55,56}$ In addition, the fibrils are found to be capable of flocculating emulsion by depletion interactions. ${ }^{57}$ Despite their multi-functional properties, only limited research has been performed to investigate the behaviour of fibrils in more complex systems. 


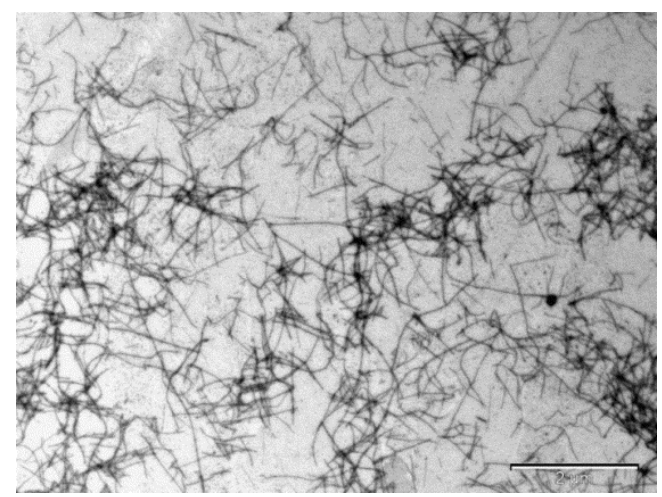

Figure 1 A TEM image of WPI fibrils (4 wt\% WPI) heated at $80^{\circ} \mathrm{C}$ for 22 hours at $\mathrm{pH} 2$, the scale bar corresponds to $2 \mu \mathrm{m}$.

\subsubsection{Bacterial cellulose $(B C)$ and $B C$ microfibrils}

As one of the most abundant biopolymers in nature, cellulose has been a subject to a large number of studies and their applications have been identified in numerous fields, as is reviewed previously. ${ }^{58,59}$ Cellulose is composed of a linear chain of glucose molecules and has a flat ribbon-like structure. Cellulose can be obtained from different sources, such as wood, plants and bacteria. ${ }^{58}$ Various treatments such as chemical surface modification ${ }^{60}$ and addition of an adsorbing polymer ${ }^{61}$ have been explored to obtain better waterdispersible dispersions; as a consequence, modified molecular structures of cellulose were developed. ${ }^{58,59}$ Carboxymethyl cellulose (CMC) and hydroxypropyl methylcellulose (HPMC) are common cellulose derivatives used in foods. ${ }^{62}$

Microfibrillated cellulose is made from cellulose fibers using high-pressure homogenization and has been shown to have wide applications in foods. ${ }^{63-67}$ The microfibrils are present in the form of microfibrillar aggregates and form an entangled network with a gel-like behaviour. ${ }^{68,69}$ Microfibrillated cellulose from plants or wood is often not pure, due to the presence of lignin, pectin, hemicellulose etc.

In contrast to plant or wood derived cellulose, bacterial cellulose (BC), derived from a bacterial source, is a pure form of cellulose and regarded as a model system. ${ }^{70} \mathrm{BC}$ is produced mainly by Gluconacetobacter strains, in the form of ribbon-like microfibrils. ${ }^{71}$ The microfibrils have a width of approximately $60 \mathrm{~nm}$, a thickness about $9 \mathrm{~nm}$ and length 
up to several micrometers. ${ }^{72}$ Due to the strong attraction between the microfibrils, they are present in the form of microfibrillar bundles that agglomerate into a space-spanning network, leading to a gel-like behaviour. The surface charge of $\mathrm{BC}$ resulting from the hydroxyl groups is low and the streaming potential is reported to be around $-7.5 \mathrm{mV}{ }^{73}$ which is too low to stabilize the microfibrils by electrostatic repulsion. The unique nanosized fibrillar structures endow BC with remarkable physical and mechanical properties in comparison to plant cellulose and offer broad potential applications in fields of food, cosmetics, biomedical, optics and paper-making etc. ${ }^{74-79}$ The popular dessert in the Phillipines, Nata de Coco, ${ }^{80}$ contains mainly BC and water. Functionalities of BC in foods range from thickening, gelling, emulsifying, and stabilizing to water binding agents. ${ }^{77}$ ${ }^{81-90}$ Moreover, BC offers the health benefits of a dietary fiber ${ }^{91}$ and can be used as a fat substitute. ${ }^{89,90}$ Despite its suitability as a functional ingredient in foods, the research on its interactions with other components, like proteins or emulsion droplets is rather limited. ${ }^{83,}$ ${ }^{84,86}$ The majority of the research on BC has been focused on its formation mechanism, production and characterization of the pellicles. ${ }^{58}$

Recently, BC microfibril dispersions (Figure 2) have been prepared by extracting BC from the commercially available source 'Nata de Coco' through a high-energy mechanical deagglomeration process. ${ }^{70}$ Due to the strong inter-fibrillar attraction, the microfibrils readily re-agglomerate and thus form a heterogeneous mixture, containing bundles of microfibrils, flocs and voids. The gelation threshold of the BC microfibrils based on a theoretical estimate is $0.1 \mathrm{wt}^{70}{ }^{70}$ Adding sodium carboxymethyl cellulose (CMC) in BC microfibril dispersions, and subsequently applying the high energy de-agglomeration process was reported to result in a more homogeneous dispersion, presumably due to the adsorption of CMC on the BC microfibril surface. ${ }^{92,93}$ 


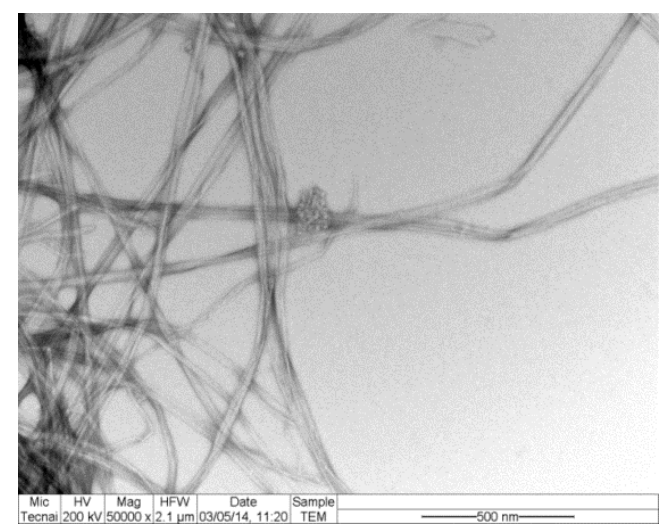

Figure 2 A TEM image of BC microfibrils $(0.25 \mathrm{wt} \%)$ treated by high-energy mechanical deagglomeration. The scale bar corresponds to $500 \mathrm{~nm}$.

\subsubsection{Proteins and BC microfibrils}

It is of interest to study the interactions between proteins and BC microfibrils under different conditions (e.g. protein aggregation or fibril formation) because of the potential applications of BC to food systems. Since BC is also a polysaccharide, therefore, we first introduce the area of protein and polysaccharide interactions. In general, proteins and polysaccharides are biopolymers commonly present in foods. They serve as the main structuring agents and both contribute to the stability, structure and texture of the food products. Their well-known functionalities include thickening, gelling, emulsifying, and stabilizing properties. ${ }^{94-99}$ For example, mixtures of proteins and polysaccharides are often used in emulsions to confer long-term stability. ${ }^{95-97,100,101}$ When mixing proteins and polysaccharides, complicated behaviour can occur depending on concentrations, $\mathrm{pH}$, ionic strength and temperature. ${ }^{102-104}$ The main phenomena that can occur are thermodynamic incompatibility, co-solubility and complexation, leading to one-phase or two-phase systems. ${ }^{102,105,106}$ Thermodynamic incompatibility is a general phenomenon in biopolymer mixtures, where most protein and polysaccharide mixtures tend to phase separate. ${ }^{102,106 \text {, }}$ 107

Gelation of protein and polysaccharide mixtures is an important subject that has been received considerable attention in literature. ${ }^{99,106,108-113}$ Depending on the conditions used, their gels vary in structural and rheological properties. In general, the gel structures can be 
classified into connected, interpenetrating and phase separated gels. ${ }^{98}$ The interpenetrating gels consist of two (or more) networks that are spanning the entire system. Apart from the extensive studies on different mixed aqueous solutions of proteins and polysaccharides, research on interactions between proteins and polysaccharides has been reviewed by a number of authors. ${ }^{97-99,102-108,114-119}$ However, limited work has been performed on interactions between proteins and $\mathrm{BC}$ microfibrils, and on protein fibrils and $\mathrm{BC}$ microfibrils. Due to the relevance of these systems to possible novel applications, these interactions form part of the current thesis.

\subsubsection{Dispersions and emulsions containing fibrillar structures}

Dispersions of spherical droplets or particles are present in many products, i.e. emulsions, foams and polystyrene latex dispersions. Their applications include foods, cosmetics and paints. ${ }^{120}$ Their stability has been of interest for a long time due to its importance to fundamental understanding and industrial applications. To confer long-term stability to dispersions, polymers like proteins and/or polysaccharides are often added. Mixing of spherical droplets or particles with polymers often give rise to a rich phase behaviour. For example, flocculation of dispersions upon the addition of polymer can be often observed, ${ }^{121}$ which can be caused by either bridging flocculation or depletion flocculation. Bridging flocculation takes place when the polymer adsorbs to the droplets or particles, as a result of their opposite charge. ${ }^{122,123}$ Bridging typically occurs when the amount of polymer is not sufficient to fully cover the surface of the colloids. On the other hand, the addition of non-adsorbing polymers to emulsions or dispersions can cause depletion flocculation. $^{124,125}$

The addition of polymers to dispersions confers long-term stability due to various stabilization mechanisms. For example, electrostatic and steric stabilization are wellknown mechanisms in colloidal dispersions. ${ }^{126,127}$ Stabilization by a viscosity increase and/or gelation of the continuous phase, as well as the formation of a network by the colloids themselves has also been reported. ${ }^{95,128,129}$ Depletion stabilization, initially proposed by Feigin and Napper ${ }^{130,131}$ was attributed to a kinetic barrier between the colloids and the polymer, when they are in close proximity. The mechanism was later argued to be thermodynamic origin by several other researchers. ${ }^{132-140}$ Although the 
discussion has been put forward by many other authors, the exact mechanism still remains a subject of debate. ${ }^{141-152}$

Due to their large excluded volume, rod-like particles are weight effective depletion agents. ${ }^{153}$ They are known to induce rich phase behaviour when mixed with spherical particles and droplets, and these systems have been studied extensively, both theoretically and experimentally. ${ }^{120,154-156}$ However, the case of rod-like protein fibrils and/or cellulose microfibrils in combination with emulsions or dispersions has received rather limited attention. ${ }^{57,83,84}$

\subsection{Aim and outline of the thesis}

This thesis focuses on mixtures of fibrillar and spherical structures. The fibrillar structures that are studied are BC microfibrils and protein fibrils made from WPI. The spherical structures that are used are polystyrene latex particles, whey protein particles and emulsion droplets. The aim is to investigate how the fibrillar structures interact with one another, and how the fibrillar structures interact with the spherical structures in the mixed systems. By understanding these interactions, one can control the stability, microstructure and rheological properties of systems containing both spherical and fibrillar structurres and thereby target for specific applications.

The outline of the thesis is schematically represented in the form of a ternary phase diagram (cf. Figure 3) where the three components are the bacterial cellulose microfibrils, the whey protein isolate and the spherical particles. Each point in the triangle corresponds to a particular mixture of the three components studied. In this thesis, the stability, structural, and rheological properties of the mixtures as indicated are studied. 


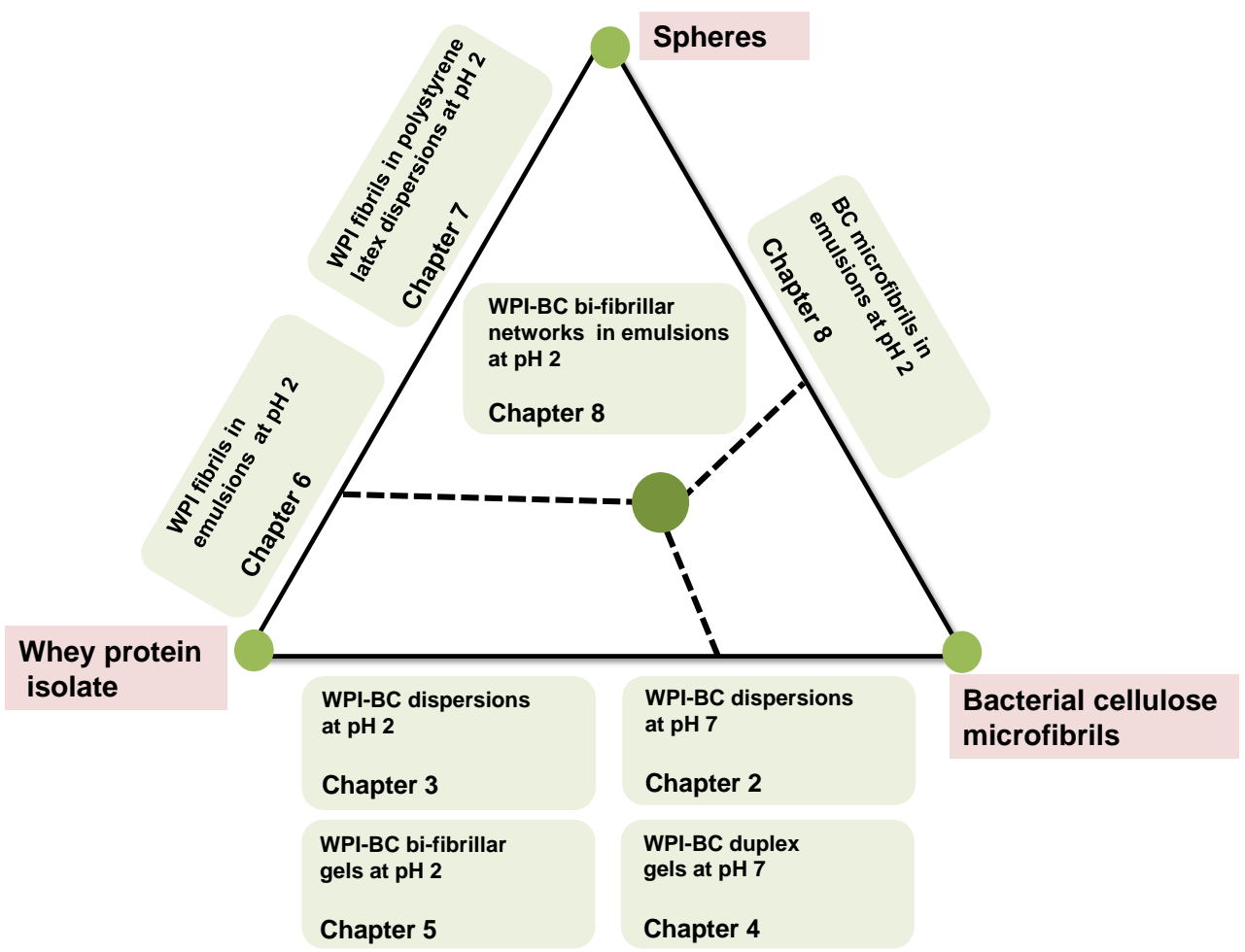

Figure 3 Schematic representation of the outline of the studies in the thesis

In Chapter 2, we investigated the mixed dispersions containing bacterial cellulose microfibrils and whey protein isolate at neutral $\mathrm{pH}(\mathrm{pH} 7)$, in which WPI carries negative charges. The concentration ratios between WPI and BC microfibrils were varied. The mixtures were further heated to investigate their interactions during heating, in which WPI undergoes denaturation and aggregation. The stability, rheological and microstructural properties of both the unheated and heated mixtures are characterized to investigate the specific interactions between WPI and BC microfibrils at neutral $\mathrm{pH}$.

Chapter 3 investigated the WPI fibril formation in the presence of $\mathrm{BC}$ microfibrils at $\mathrm{pH} 2$ upon prolonged heating. The aim is to investigate the effect of $B C$ microfibrils on the protein fibril formation of WPI. WPI fibrils formed in the presence of BC microfibrils were compared to the WPI fibrils formed separately. Both concentrations of WPI and BC 
microfibrils were varied in the mixtures. The mixtures were characterized by means of their rheological and microstructural properties.

Chapter 4 investigated the heat-induced mixed gels prepared from WPI and BC microfibrils at $\mathrm{pH}$ 7. Visco-elastic properties, microstructure, mechanical properties and water holding capacity of the mixed gels were determined. The aim is to investigate the effect of $B C$ microfibrils on the gelation of WPI at $\mathrm{pH} 7$ under different $\mathrm{BC}$ microfibril concentrations and $\mathrm{NaCl}$ concentrations.

The aim of Chapter $\mathbf{5}$ is to study a mixed gel composed of two fibrillar gels, i.e. a bi-fibrillar gel. A mixture of WPI and $\mathrm{BC}$ microfibrils was heated at $\mathrm{pH} 2$ for 10 hours at $80^{\circ} \mathrm{C}$, during which the WPI fibrils were formed. A range of $B C$ microfibril concentrations were added to the WPI solution to investigate the effect of $B C$ microfibril concentration on the formation of WPI fibrils. The properties of the mixed gels were characterized in terms of their rheological, microstructural and mechanical properties and were compared to the WPI fibrillar gels formed separately.

Chapter 6 investigated the stability of a WPI-stabilized o/w emulsion in the presence of WPI fibrils at $\mathrm{pH} 2$. Here both emulsion droplets and protein fibrils are positively charged. The stability of the emulsions containing WPI fibrils were studied using rheology, diffusing wave spectroscopy and confocal laser scanning microscopy. The effect of the volume fraction of oil droplets, fibril length distribution and temperature on the emulsion stability was also studied. The aim is to study emulsion stability over a wide range of WPI fibril concentrations.

The stability of monodispersed polystyrene latex dispersions containing WPI fibrils at $\mathrm{pH} 2$ was investigated in Chapter 7. Here the latex particles and proteins fibrils are oppositely charged. This is in contrast to the study in Chapter 6 where the oil droplets and WPI fibrils carry the same charge. The size and the zeta potential of the latex particles were varied. The stability of the latex dispersions was studied using microscopy and diffusing wave spectroscopy. The influence of temperature on dispersion stability was also studied.

In Chapter 8, the stability of a WPI-stabilized o/w emulsion containing a bi-fibrillar networks consisting of both WPI fibrils and $\mathrm{BC}$ microfibrils at $\mathrm{pH} 2$ was studied. The effect of pure $B C$ microfibrils on the emulsion stability was studied and compared to the emulsions in the presence of bi-fibrillar network. The effect of temperature, $\mathrm{BC}$ microfibril 
Chapter 1

concentration and salt concentration on the emulsion stability containing bi-fibrillar network was also studied. The emulsion stability was characterized using rheology and microscopy.

A general discussion of the results presented in this thesis is given in Chapter 9. Also recommendations for future work and concluding remarks are given. 


\subsection{References}

1. C. Akkermans, A. J. van der Goot, P. Venema, E. van der Linden and R. M. Boom, Food Hydrocolloids, 2008, 22, 1315-1325.

2. L. N. Arnaudov and R. de Vries, Biophysical Journal, 2005, 88, 515-526.

3. C. Veerman, G. de Schiffart, L. M. C. Sagis and E. van der Linden, International Journal of Biological Macromolecules, 2003, 33, 121-127.

4. C. Akkermans, A. J. Van der Goot, P. Venema, H. Gruppen, J. M. Vereijken, E. Van der Linden and R. M. Boom, Journal of Agricultural and Food Chemistry, 2007, 55, 9877-9882.

5. C. D. Munialo, A. H. Martin, E. van der Linden and H. H. J. de Jongh, Journal of Agricultural and Food Chemistry, 2014, 62, 2418-2427.

6. A. Kroes-Nijboer, P. Venema and E. van der Linden, Food \& function, 2012, 3, 221-227.

7. G. W. Smithers, International dairy journal, 2008, 18, 695-704.

8. C. V. Morr and E. Y. W. Ha, Critical Reviews in Food Science and Nutrition, 1993, 33, 431-476.

9. D. Mulvihill and J. Kinsella, Food technology (USA), 1987.

10. J. De Wit, Journal of Dairy Science, 1998, 81, 597-608.

11. T. Nicolai, M. Britten and C. Schmitt, Food Hydrocolloids, 2011, 25, 1945-1962.

12. T. Nicolai and D. Durand, Current Opinion in Colloid \& Interface Science, 2013, 18, 249-256.

13. A. Clark, G. Kavanagh and S. Ross-Murphy, Food Hydrocolloids, 2001, 15, 383-400.

14. P. B. Fernandes, Food Hydrocolloids, 1994, 8, 277-285.

15. W. Chantrapornchai and D. J. McClements, Food Hydrocolloids, 2002, 16, 467-476.

16. E. A. Foegeding, E. L. Bowland and C. C. Hardin, Food Hydrocolloids, 1995, 9, 237-249.

17. M. Langton and A.-M. Hermansson, Food Hydrocolloids, 1992, 5, 523-539.

18. J. M. Aguilera, Food technology, 1995, 49, 83-89.

19. S. Barbut, Food Research International, 1995, 28, 437-443.

20. J. I. Boye, I. Alli, A. A. Ismail, B. F. Gibbs and Y. Konishi, International dairy journal, 1995, 5, 337353.

21. A. Brodkorb, T. Croguennec, S. Bouhallab and J. J. Kehoe, in Advanced Dairy Chemistry, Springer, 2016, pp. 155-178.

22. D. Durand, J. C. Gimel and T. Nicolai, Physica A: Statistical Mechanics and its Applications, 2002, 304, 253-265.

23. E. A. Foegeding and J. P. Davis, Food Hydrocolloids, 2011, 25, 1853-1864.

24. E. A. Foegeding, J. P. Davis, D. Doucet and M. K. McGuffey, Trends in Food Science \& Technology, 2002, 13, 151-159.

25. S. Ikeda, E. A. Foegeding and T. Hagiwara, Langmuir, 1999, 15, 8584-8589.

26. S. Ikeda and V. J. Morris, Biomacromolecules, 2002, 3, 382-389.

27. L. L. Lowe, E. A. Foegeding and C. R. Daubert, Food Hydrocolloids, 2003, 17, 515-522. 
28. D. M. Mulvihill and M. C. F. p. d. Donovan, Irish Journal of Food Science and Technology, 1987, 11, 43-75.

29. M. Verheul and S. P. Roefs, Journal of Agricultural and Food Chemistry, 1998, 46, 4909-4916.

30. S. G. Bolder, A. J. Vasbinder, L. M. C. Sagis and E. van der Linden, International dairy journal, 2007, 17, 846-853.

31. C. Akkermans, P. Venema, A. J. van der Goot, H. Gruppen, E. J. Bakx, R. M. Boom and E. van der Linden, Biomacromolecules, 2008, 9, 1474-1479.

32. L. M. C. Sagis, C. Veerman and E. van der Linden, Langmuir, 2004, 20, 924-927.

33. L. N. Arnaudov, R. de Vries, H. Ippel and C. P. M. van Mierlo, Biomacromolecules, 2003, 4, 16141622.

34. G. M. Kavanagh, A. H. Clark and S. B. Ross-Murphy, International Journal of Biological Macromolecules, 2000, 28, 41-50.

35. W. S. Gosal, A. H. Clark, P. D. A. Pudney and S. B. Ross-Murphy, Langmuir, 2002, 18, 7174-7181.

36. J. Adamcik, J.-M. Jung, J. Flakowski, P. De Los Rios, G. Dietler and R. Mezzenga, 2010, 5, 423428.

37. C. Lara, J. Adamcik, S. Jordens and R. Mezzenga, Biomacromolecules, 2011, 12, 1868-1875.

38. S. Bolisetty, J. Adamcik and R. Mezzenga, Soft Matter, 2011, 7, 493-499.

39. S. Loveday, X. Wang, M. Rao, S. Anema and H. Singh, Journal of Agricultural and Food Chemistry, 2011, 59, 8467-8474.

40. S. M. Loveday, J. Su, M. A. Rao, S. G. Anema and H. Singh, International dairy journal, 2012, 26, 133-140.

41. S. Loveday, X. Wang, M. Rao, S. Anema, L. Creamer and H. Singh, International dairy journal, 2010, 20, 571-579.

42. C. Akkermans, A. J. Van der Goot, P. Venema, E. Van der Linden and R. M. Boom, International dairy journal, 2008, 18, 1034-1042.

43. P. Aymard, T. Nicolai, D. Durand and A. Clark, Macromolecules, 1999, 32, 2542-2552.

44. S. G. Bolder, H. Hendrickx, L. M. C. Sagis and E. van der Linden, Journal of Agricultural and Food Chemistry, 2006, 54, 4229-4234.

45. S. Bolisetty, L. Harnau, J.-m. Jung and R. Mezzenga, Biomacromolecules, 2012, 13, 3241-3252.

46. W. S. Gosal, A. H. Clark and S. B. Ross-Murphy, Biomacromolecules, 2004, 5, 2408-2419.

47. A. Kroes-Nijboer, H. Sawalha, P. Venema, A. Bot, E. Flöter, R. den Adel, W. G. Bouwman and E. van der Linden, Faraday Discussions, 2012, 158, 125-138.

48. R. Mezzenga, J.-M. Jung and J. Adamcik, Langmuir, 2010, 26, 10401-10405.

49. J.-M. Jung and R. Mezzenga, Langmuir, 2010, 26, 504-514.

50. C. Veerman, L. M. C. Sagis and E. van der Linden, Macromolecular Bioscience, 2003, 3, 243-247.

51. W. S. Gosal, A. H. Clark and S. B. Ross-Murphy, Biomacromolecules, 2004, 5, 2420-2429.

52. G. M. Kavanagh, A. H. Clark and S. B. Ross-Murphy, Langmuir, 2000, 16, 9584-9594. 
53. S. M. Loveday, M. A. Rao, L. K. Creamer and H. Singh, Journal of Food Science, 2009, 74, R47R55.

54. L. M. C. Sagis, C. Veerman, R. Ganzevles, M. Ramaekers, S. G. Bolder and E. van der Linden, Food Hydrocolloids, 2002, 16, 207-213.

55. K. N. P. Humblet-Hua, G. Scheltens, E. van der Linden and L. M. C. Sagis, Food Hydrocolloids, 2011, 25, 569-576.

56. N. P. K. Humblet-Hua, E. van der Linden and L. M. C. Sagis, Journal of Agricultural and Food Chemistry, 2012, 60, 9502-9511.

57. T. B. J. Blijdenstein, C. Veerman and E. van der Linden, Langmuir, 2004, 20, 4881-4884.

58. R. J. Moon, A. Martini, J. Nairn, J. Simonsen and J. Youngblood, Chemical Society Reviews, 2011, 40, 3941-3994.

59. A. Dufresne, Materials Today, 2013, 16, 220-227.

60. T. Saito, Y. Nishiyama, J.-L. Putaux, M. Vignon and A. Isogai, Biomacromolecules, 2006, 7, 16871691.

61. A. Sorvari, T. Saarinen, S. Haavisto, J. Salmela, M. Vuoriluoto and J. Seppälä, Carbohydrate Polymers, 2014, 106, 283-292.

62. J. Murray, G. Phillips and P. Williams, Handbook of hydrocolloids, 2009, 710-723.

63. F. W. Herrick, R. L. Casebier, J. K. Hamilton and K. R. Sandberg, Microfibrillated cellulose: morphology and accessibility, 1983.

64. M. P. Lowys, J. Desbrières and M. Rinaudo, Food Hydrocolloids, 2001, 15, 25-32.

65. A. F. Turbak, F. W. Snyder and K. R. Sandberg, Microfibrillated cellulose, a new cellulose product: properties, uses, and commercial potential, 1983.

66. I. Siró and D. Plackett, Cellulose, 2010, 17, 459-494.

67. H. P. S. Abdul Khalil, Y. Davoudpour, M. N. Islam, A. Mustapha, K. Sudesh, R. Dungani and M. Jawaid, Carbohydrate Polymers, 2014, 99, 649-665.

68. G. Agoda-Tandjawa, S. Durand, S. Berot, C. Blassel, C. Gaillard, C. Garnier and J. L. Doublier, Carbohydrate Polymers, 2010, 80, 677-686.

69. M. Pääkkö, M. Ankerfors, H. Kosonen, A. Nykänen, S. Ahola, M. Österberg, J. Ruokolainen, J. Laine, P. T. Larsson, O. Ikkala and T. Lindström, Biomacromolecules, 2007, 8, 1934-1941.

70. A. Kuijk, R. Koppert, P. Versluis, G. van Dalen, C. Remijn, J. Hazekamp, J. Nijsse and K. P. Velikov, Langmuir, 2013, 29, 14356-14360.

71. R. E. Cannon and S. M. Anderson, Critical Reviews in Microbiology, 1991, 17, 435-447.

72. U. Geyer, T. Heinze, A. Stein, D. Klemm, S. Marsch, D. Schumann and H. P. Schmauder, International Journal of Biological Macromolecules, 1994, 16, 343-347.

73. K.-Y. Lee, F. Quero, J. J. Blaker, C. A. S. Hill, S. J. Eichhorn and A. Bismarck, Cellulose, 2011, 18, 595-605.

74. M. Iguchi, S. Yamanaka and A. Budhiono, Journal of Materials Science, 35, 261-270. 
75. R. Jonas and L. F. Farah, Polymer Degradation and Stability, 1998, 59, 101-106.

76. B. V. Mohite and S. V. Patil, Biotechnology and Applied Biochemistry, 2014, 61, 101-110.

77. Z. Shi, Y. Zhang, G. O. Phillips and G. Yang, Food Hydrocolloids, 2014, 35, 539-545.

78. A. Svensson, E. Nicklasson, T. Harrah, B. Panilaitis, D. L. Kaplan, M. Brittberg and P. Gatenholm, Biomaterials, 2005, 26, 419-431.

79. N. Shah, M. UI-Islam, W. A. Khattak and J. K. Park, Carbohydrate Polymers, 2013, 98, 1585-1598.

80. M. Phisalaphong and N. Chiaoprakobkij, Bacterial Cellulose: A Sophisticated Multifunctional Material, 2012, 9, 143-156.

81. A. Okiyama, M. Motoki and S. Yamanaka, Food Hydrocolloids, 1992, 6, 479-487.

82. A. Okiyama, M. Motoki and S. Yamanaka, Food Hydrocolloids, 1993, 6, 503-511.

83. H. Ougiya, K. Watanabe, Y. Morinaga and F. Yoshinaga, Bioscience, Biotechnology, and Biochemistry, 1997, 61, 1541-1545.

84. P. Paximada, A. A. Koutinas, E. Scholten and I. G. Mandala, Food Hydrocolloids, 2016, 54, 245254.

85. A. Okiyama, M. Motoki and S. Yamanaka, Food Hydrocolloids, 1993, 6, 493-501.

86. P. Paximada, E. Tsouko, N. Kopsahelis, A. A. Koutinas and I. Mandala, Food Hydrocolloids, 2016, 53, 225-232.

87. S.-Y. Yu and K.-W. Lin, Journal of Food Science, 2014, 79, C1117-C1122.

88. H. Zhu, S. Jia, H. Yang, W. Tang, Y. Jia and Z. Tan, Food Science and Biotechnology, 2010, 19, 1479-1484.

89. S.-B. Lin, L.-C. Chen and H.-H. Chen, Journal of Food Process Engineering, 2011, 34, 1363-1379.

90. K. W. Lin and H. Y. Lin, Journal of Food Science, 2004, 69, SNQ107-SNQ111.

91. S. Cho and N. Almeida, Dietary fiber and health, CRC Press, 2012.

92. S. J. Veen, A. Kuijk, P. Versluis, H. Husken and K. P. Velikov, Langmuir, 2014, 30, 13362-13368.

93. S. J. Veen, P. Versluis, A. Kuijk and K. P. Velikov, Soft Matter, 2015, 11, 8907-8912.

94. E. Dickinson, Trends in Food Science \& Technology, 1998, 9, 347-354.

95. E. Dickinson, Food Hydrocolloids, 2003, 17, 25-39.

96. E. Dickinson, Food Hydrocolloids, 2009, 23, 1473-1482.

97. E. Dickinson and V. B. Galazka, Gums and stabilisers for the food industry, 1992, 6, 351-362.

98. V. Morris, Gums and stabilisers for the food industry, 1986, 3, e99.

99. D. V. Zasypkin, E. E. Braudo and V. B. Tolstoguzov, Food Hydrocolloids, 1997, 11, 159-170.

100. E. Dickinson and S. R. Euston, Stability of food emulsions containing both protein and polysaccharide, Royal Society of Chemistry: Cambridge, UK, 1991.

101. M. Evans, I. Ratcliffe and P. A. Williams, Current Opinion in Colloid \& Interface Science, 2013, 18, 272-282.

102. V. Tolstoguzov, Food Hydrocolloids, 1991, 4, 429-468. 
103. S. Turgeon, M. Beaulieu, C. Schmitt and C. Sanchez, Current Opinion in Colloid \& Interface Science, 2003, 8, 401-414.

104. C. G. de Kruif, F. Weinbreck and R. de Vries, Current Opinion in Colloid \& Interface Science, 2004, 9, 340-349.

105. C. G. De Kruif and R. Tuinier, Food Hydrocolloids, 2001, 15, 555-563.

106. J.-L. Doublier, C. Garnier, D. Renard and C. Sanchez, Current Opinion in Colloid \& Interface Science, 2000, 5, 202-214.

107. V. Y. Grinberg and V. Tolstoguzov, Food Hydrocolloids, 1997, 11, 145-158.

108. S. K. Samant, R. S. Singhal, P. R. Kulkarni and D. V. Rege, International Journal of Food Science \& Technology, 1993, 28, 547-562.

109. V. Tolstoguzov, Functional properties of food macromolecules, 1998, 252.

110. V. B. Tolstoguzov, FOOD SCIENCE AND TECHNOLOGY-NEW YORK-MARCEL DEKKER-, 1997, 171198.

111. C. Schmitt, C. Sanchez, S. Desobry-Banon and J. Hardy, Critical Reviews in Food Science and Nutrition, 1998, 38, 689-753.

112. H. Firoozmand and D. Rousseau, Food Hydrocolloids, 2015, 50, 84-93.

113. S. Banerjee and S. Bhattacharya, Critical Reviews in Food Science and Nutrition, 2012, 52, 334346.

114. K. K. T. Goh, A. Sarkar and H. Singh, in Milk Proteins, 2008, pp. 347-376.

115. D. J. McClements, Biotechnology Advances, 2006, 24, 621-625.

116. V. I. Polyakov, V. Y. Grinberg and V. B. Tolstoguzov, Food Hydrocolloids, 1997, 11, 171-180.

117. A. P. Imeson, D. A. Ledward and J. R. Mitchell, Journal of the Science of Food and Agriculture, 1977, 28, 661-668.

118. V. Tolstoguzov, D. E. B. Harry Walter and A. S. Paul, in International Review of Cytology, Academic Press, 1999, vol. Volume 192, pp. 3-31.

119. A. A. Perez, C. R. Carrara, C. C. Sánchez, J. M. Rodríguez Patino and L. G. Santiago, Food Chemistry, 2009, 116, 104-113.

120. H. N. W. Lekkerkerker and R. Tuinier, Colloids and the depletion interaction, Springer, Dordrecht ; New York, 2011.

121. D. H. Napper, Polymeric stabilization of colloidal dispersions, Academic Pr, 1983.

122. E. Pelssers, M. C. Stuart and G. Fleer, Colloids and Surfaces, 1989, 38, 15-25.

123. E. Dickinson and L. Eriksson, Advances in Colloid and Interface Science, 1991, 34, 1-29.

124. S. Asakura and F. Oosawa, The Journal of Chemical Physics, 1954, 22, 1255-1256.

125. S. Asakura and F. Oosawa, Journal of Polymer Science, 1958, 33, 183-192.

126. D. H. Napper, Journal of Colloid and Interface Science, 1977, 58, 390-407.

127. B. Vincent, Advances in Colloid and Interface Science, 1974, 4, 193-277.

128. E. Dickinson, SPECIAL PUBLICATION-ROYAL SOCIETY OF CHEMISTRY, 1993, 113, 77-77. 
129. A. Parker, P. A. Gunning, K. Ng and M. M. Robins, Food Hydrocolloids, 1995, 9, 333-342.

130. R. I. Feigin and D. H. Napper, Journal of Colloid and Interface Science, 1980, 74, 567-571.

131. R. I. Feigin and D. H. Napper, Journal of Colloid and Interface Science, 1980, 75, 525-541.

132. A. P. Gast and L. Leibler, Macromolecules, 1986, 19, 686-691.

133. A. P. Gast and L. Leibler, The Journal of Physical Chemistry, 1985, 89, 3947-3949.

134. G. Fleer and J. Scheutjens, Croatica chemica acta, 1987, 60, 477-494.

135. G. J. Fleer, J. H. M. H. Scheutjens and B. Vincent, in Polymer Adsorption and Dispersion Stability, American Chemical Society, 1984, vol. 240, pp. 245-263.

136. J. M. H. M. Scheutjens and G. J. Fleer, Advances in Colloid and Interface Science, 1982, 16, 361380 .

137. B. Vincent, J. Edwards, S. Emmett and A. Jones, Colloids and Surfaces, 1986, 18, $261-281$.

138. J. Clarke and B. Vincent, Journal of Colloid and Interface Science, 1981, 82, 208-216.

139. J. Clarke and B. Vincent, Journal of the Chemical Society, Faraday Transactions 1: Physical Chemistry in Condensed Phases, 1981, 77, 1831-1843.

140. B. Vincent, P. F. Luckham and F. A. Waite, Journal of Colloid and Interface Science, 1980, 73, 508-521.

141. J. Groenewold and W. K. Kegel, The Journal of Physical Chemistry B, 2001, 105, 11702-11709.

142. N. Dutta and D. Green, Langmuir, 2008, 24, 5260-5269.

143. J. Liu, W. Y. Shih, R. Kikuchi and I. A. Aksay, Journal of Colloid and Interface Science, 1991, 142, 369-377.

144. J. Y. Walz and A. Sharma, Journal of Colloid and Interface Science, 1994, 168, 485-496.

145. A. L. Ogden and J. A. Lewis, Langmuir, 1996, 12, 3413-3424.

146. S. Kim, K. Hyun, J. Y. Moon, C. Clasen and K. H. Ahn, Langmuir, 2015, 31, 1892-1900.

147. M. Yasrebi, W. Y. Shih and I. A. Aksay, Journal of Colloid and Interface Science, 1991, 142, 357368 .

148. A. Sharma, S. N. Tan and J. Y. Walz, Journal of Colloid and Interface Science, 1997, 190, $392-407$.

149. Y. Mao, M. E. Cates and H. N. W. Lekkerkerker, Physica A: Statistical Mechanics and its Applications, 1995, 222, 10-24.

150. X. Zhang, M. R. Servos and J. Liu, Journal of the American Chemical Society, 2012, 134, 99109913.

151. Y. Mao, M. E. Cates and H. N. W. Lekkerkerker, Physical Review Letters, 1995, 75, 4548-4551.

152. Y.-L. Chen and K. S. Schweizer, The Journal of Chemical Physics, 2002, 117, 1351-1362.

153. A. P. Philipse, Langmuir, 1996, 12, 1127-1133.

154. M. Adams, Z. Dogic, S. L. Keller and S. Fraden, Nature, 1998, 393, 349-352.

155. K.-h. Lin, J. C. Crocker, A. C. Zeri and A. G. Yodh, Physical Review Letters, 2001, 87, 088301.

156. G. Vliegenthart and H. Lekkerkerker, The Journal of Chemical Physics, 1999, 111, 4153-4157. 


\section{Chapter 2}

Rheology and microstructure of

dispersions of whey proteins and cellulose microfibrils

Submitted as:

Jinfeng Peng, Vincenzo Calabrese, Sandra J. Veen, Peter Versluis, Krassimir P. Velikov, Paul Venema, Erik van der Linden. Rheology and microstructure of dispersions of whey proteins and cellulose microfibrils. 


\begin{abstract}
The rheological and microstructural properties of dispersions containing mixtures of whey protein isolate (WPI) and bacterial cellulose (BC) microfibrils were studied before and after heating at $\mathrm{pH}$ 7. Results showed that mixtures of WPI and $B C$ microfibrils form stable dispersions at $\mathrm{BC}$ microfibril concentration of $0.2 \mathrm{wt} \%$ and higher. The viscosity of the dispersions was mainly controlled by $\mathrm{BC}$ microfibrils, in which two shear-thinning regions and a viscosity plateau were observed. Heat treatment did not significantly influence the rheological behaviour of the dispersions, apart from the observation that the viscosity plateau became less pronounced at a WPI concentration of $9 \mathrm{wt} \%$. The WPI fine-stranded structures that formed upon heating were not affected by the presence of $\mathrm{BC}$ microfibrils, and vice versa, the morphology of $\mathrm{BC}$ microfibrils did not show distinct changes upon heating in the presence of WPI. Heating the WPI and BC microfibrils both together and separately prior to measurement gave identical flow curves and microstructure of the mixtures, indicating that the WPI and BC microfibrils did not show specific interactions when mixing and when heating is subsequently applied. The WPI proteins denatured and aggregated in the mixtures in the same way as when heated alone.
\end{abstract}




\subsection{Introduction}

Proteins and polysaccharides are main structuring agents in foods, which together determine final properties like stability and texture of many types of foods. ${ }^{1}$ When mixing protein and polysaccharide solutions, often phase separation is observed (at high enough concentrations)..$^{2-5}$ In order to understand this phase separation, the interactions and phase behaviour of protein and polysaccharide under different conditions have been studied extensively, as is clear from articles on specific systems ${ }^{6-17}$ and from review articles. $^{2,4,5,18-27}$

One type of polysaccharides is cellulose. Cellulose is one of the most abundant biopolymers in nature. ${ }^{28}$ Cellulose dispersions obtained from a high-pressure homogenization process (i.e. mechanical disintegration) are referred to as micro-fibrillated cellulose (MFC) and have been demonstrated to have wide application potential in foods. ${ }^{29-31}$ The micro-fibrillated cellulose forms a highly entangled network consisting of micro-fibrillar aggregates and have a gel-like behaviour due to their strong interfibrillar attractive forces. ${ }^{32}$ Different polymers have been added to these cellulose dispersions to modify their rheological properties and/or improve their stability against sedimentation. ${ }^{31}$ 33-35 So far research on micro-fibrillated cellulose has been mainly focused on wood and plant-derived cellulose. Recently, bacterial cellulose (BC) has received increasing attention as a food functional ingredient due to its multi-functionality (e.g. thickening, gelling and stabilizing). ${ }^{36} \mathrm{BC}$ can be used as a model system for plant-derived cellulose due to its high purity, i.e. without the presence of lignin, pectin or hemicellulose. ${ }^{37}$ Recently, the microfibrillated cellulose from bacterial cellulose (BC) extracted from Nata de Coco has been prepared using high-energy mechanical de-agglomeration process. ${ }^{38,}{ }^{39}$ Rheological properties and the microstructure of the resulting dispersions have been characterized. The $B C$ is present in the dispersion as micro-fibrillar bundles due to strong hydrogen bonding. ${ }^{38}$ Veen et al. found that high-energy mechanical de-agglomeration of $\mathrm{BC}$ microfibrils in the presence of sodium-carboxymethyl cellulose (CMC) resulted in a better dispersibility of the $\mathrm{BC}$ microfibrils. This was attributed to the adsorption of $\mathrm{CMC}$ on the surface of BC microfibrils, thereby hindering the formation of BC micro-fibrillar bundles. ${ }^{40}$ The addition of $\mathrm{CMC}$ leads to a change of the microstructure and rheological properties of the $\mathrm{BC}$ microfibril dispersions. ${ }^{41}$ 
In this study, the interactions between whey proteins and $\mathrm{BC}$ microfibrils at $\mathrm{pH} 7$ are investigated. Whey proteins are of crucial importance to the food industry due to their nutritional and functional properties. ${ }^{42}$ The physical properties of whey proteins under different conditions have been well documented. ${ }^{43-48}$ Here we investigate whether the presence of whey proteins (in this case in the form of a specific mixture referred to as whey protein isolate, in short, WPI) modifies the rheology and microstructure of $B C$ microfibril dispersions and, reversely, whether the presence of $B C$ microfibrils affects the whey protein denaturation and aggregation upon heating.

\subsection{Materials and Methods}

\subsubsection{Solution of whey protein isolate (WPI)}

WPI powder was obtained from Davisco (Bipro, Davisco, lot \# JE 198 -1-420, USA). To prepare a 25 wt \% WPI stock solution, the powder was dissolved and stirred in MilliQ water at $4{ }^{\circ} \mathrm{C}$ for 2 days for complete hydration. The $\mathrm{pH}$ of the WPI solution was adjusted to 7 using a $3 \mathrm{M} \mathrm{NaOH}$ solution. Subsequently, the solution was filtered through a filter (Hydrophilic PES $0.45 \mu \mathrm{m}$, Millipore Millex-HP) to remove undissolved protein. To determine the protein concentration of the solution, we used an UV spectrophotometer (Cary 50 Bio, Varian) at a wavelength of $280 \mathrm{~nm}$ and a calibration curve determined from WPI-solutions at known concentrations. The stock solution was stored at $4{ }^{\circ} \mathrm{C}$ for further sample preparation.

\subsubsection{Dispersion of $\mathrm{BC}$ microfibrils}

The dispersion of $\mathrm{BC}$ microfibrils was prepared following the same procedure described elsewhere. ${ }^{38,40}$ In short, BC cubes from commercially available Nata de Coco dessert ( Kara Santan Pertama, Bogor 16964, Indonesia) was cut using a hand blender (Braun 4185545) and washed 8 times using MilliQ water. Each washing step consisted of rinsing the cellulose by filtration over a vacuum filter (Whatman Schleicher and Schuell 113, wetstrengthened circles, $185 \mathrm{~mm}$ in diameter) and redispersing the residue in $1.5 \mathrm{~L}$ of MilliQ water with the hand blender. Subsequently, the dispersion was passed once through a high pressure homogeniser (Microfluidizer ${ }^{T M}$ (MF), Microfluidics - M 110S) with a Zchamber of $87 \mu \mathrm{m}$ at a pressure of 1200 bar $\left(1.2 \times 10^{5} \mathrm{kPa}\right)$ to obtain a more 
homogeneous dispersion. The concentration of the obtained dispersion was determined by drying approximately $20 \mathrm{~g}$ of dispersion in a vacuum oven at $40 \mathrm{mbar}$ and $40{ }^{\circ} \mathrm{C}$ for 3 days. The final concentration of the stock dispersion is $1 \mathrm{wt} \%$.

\subsubsection{Mixtures of WPI and BC microfibrils}

The above stock solution of WPI and stock dispersion of BC microfibrils were used to prepare the WPI-BC microfibril mixtures. BC microfibrils were transferred using a pipette with a cut-off tip. Sample preparation was performed using MilliQ water. The $\mathrm{pH}$ of all mixtures was adjusted to $\mathrm{pH} 7$ after mixing using a $3 \mathrm{M} \mathrm{NaOH}$ solution. Three types of WPI-BC microfibril mixtures were prepared at $\mathrm{pH}$ 7. For clarity, the concentration of WPI and $\mathrm{BC}$ microfibrils both in weight percentage (wt\%) are indicated as [WPI, BC]. All concentrations refer to the final concentrations in the mixtures.

\subsubsection{Native WPI-BC microfibril mixtures}

In these mixtures, the WPI solution was mixed with BC microfibril dispersion directly prior to further analysis. The mixtures prepared were (in wt\%): $[\mathrm{WPI}, \mathrm{BC}]=[0,0.1],[0,0.2],[0$, $0.3],[3,0.1],[6,0.1],[9,0.1],[9,0.2],[9,0.3],[9,0]$.

\subsubsection{Heated WPI-BC microfibril mixtures}

To investigate the effect of heating on the mixed WPI-BC microfibril mixtures, the mixtures were heated after mixing in a heating plate at $80^{\circ} \mathrm{C}$ for $30 \mathrm{~min}$ at $\mathrm{pH}$ 7. After heating, the mixtures were cooled down to room temperature and stored at $4{ }^{\circ} \mathrm{C}$ before further analysis. The mixtures prepared were (in wt \%): $[\mathrm{WPI}, \mathrm{BC}]=[0,0.1],[3,0.1],[6$, $0.1],[9,0.1],[9,0.2],[9,0.3],[3,0],[6,0],[9,0]$.

\subsubsection{Influence of BC microfibrils on WPI denaturation and aggregation}

The BC microfibrils were mixed into a WPI solution both before and after heating the WPI solution. WPI undergoes denaturation and further aggregation upon heating at $80{ }^{\circ} \mathrm{C}$ for $30 \mathrm{~min}$ at $\mathrm{pH}$ 7. The aim of the different sample preparations is thus to investigate the influence of $B C$ microfibrils on the denaturation and aggregation of WPI during heating, vice versa, the effect of WPI denaturation and aggregation on the rheology of the WPI-BC microfibril system and microstructure of BC microfibrils. 


\section{(1) Mixing $B C$ microfibrils before heating (BH) the WPI solution}

BC microfibrils were added directly to WPI solution and mixtures were stirred for 30 min to ensure homogeneous mixing. The mixtures were then heated at $80^{\circ} \mathrm{C}$ for $30 \mathrm{~min}$ at $\mathrm{pH}$ 7. The concentrations of WPI and BC microfibrils in these mixtures were (in wt \%): [WPI, $B C]=[6,0.2],[6,0.6],[9,0.2],[9,0.6]$. Directly after heating, these mixtures were diluted two-fold using MilliQ water to allow for a correct comparison with the dispersions as obtained from (2) below. As a result, the corresponding final concentrations (wt\%) in these mixtures are: $[\mathrm{WPI}, \mathrm{BC}]=[3,0.1],[3,0.3],[4.5,0.1],[4.5,0.3]$.

\section{(2) Mixing BC microfibrils after heating (AH) the WPI solution}

The WPI solutions and BC microfibril dispersions were first heated separately at $80{ }^{\circ} \mathrm{C}$ for $30 \mathrm{~min}$ at $\mathrm{pH}$ 7. The concentration of WPI solutions being heated are 6 and $9 \mathrm{wt} \%$. The concentrations of BC microfibril dispersions being heated are 0.2 and 0.6 wt\%. After heating, the WPI solutions and BC microfibril dispersions were mixed together and stirred for $30 \mathrm{~min}$ for homogeneous mixing prior to further analysis. The final concentrations in the WPI-BC microfibril mixtures are (in wt \%): $[\mathrm{WPI}, \mathrm{BC}]=[3,0.1],[3,0.3],[4.5,0.1],[4.5$, 0.3].

All mixtures prepared above are in liquid state and samples are stirred for $30 \mathrm{~min}$ at room temperature prior to measurements.

\subsubsection{Macroscopic stability}

The stability of WPI-BC microfibril mixtures was monitored by taking images at time 0,1 and 7 days after preparation. Samples were stored at $4^{\circ} \mathrm{C}$.

\subsubsection{Viscosity}

The viscosity was measured using a stress-controlled rheometer (MCR 302, Anton Paar) equipped with a sandblasted concentric cylinder geometry (CC17/TI/S-SN38492) to minimize wall slip. To minimize the alignment of BC microfibrils before rheological characterisation, the samples were transferred into the cup using a pipette with a cut-off tip. The samples were pre-sheared at a frequency of $1 \mathrm{~Hz}$ and strain of $0.1 \%$ for $5 \mathrm{~min}$. 
Rheology and microstructure of dispersions of whey proteins and cellulose microfibrils

Subsequently, the viscosity was monitored by increasing the shear rate from 0.1 to 500 $1 / \mathrm{s}$ in $2 \mathrm{~min}$, followed by a decrease in shear rate from 500 to $0.11 / \mathrm{s}$ in $2 \mathrm{~min}$.

\subsubsection{Size distribution of WPI aggregates}

The size distribution of WPI aggregates formed after heating was measured in order to investigate the effect of BC microfibrils on WPI aggregation. Samples were first centrifuged (Hermle, Labortechnik GmbH Z 306, Germany) at a speed of 3350 RCF for 30 min at $20{ }^{\circ} \mathrm{C}$ prior to measurement. Afterwards, the WPI aggregates in the supernatant were collected for analysis and the size distribution of the protein aggregates was determined using dynamic light scattering (Zetasizer Nano, Malvern Instruments). The measurements were performed in duplicate using a cuvette $(10 \times 10 \times 45 \mathrm{~cm}$ SARSTED) with a sample volume of $1.25 \mathrm{ml}$. The obtained size distribution histograms were plotted in volume percentage as a function of particle diameter $(\mathrm{nm})$.

\subsubsection{Differential scanning calorimetry (DSC)}

A $50 \mathrm{mg}$ sample was placed in a sealed stainless steel pan and equilibrated at $20{ }^{\circ} \mathrm{C}$ (Diamond series DSC, Perkin Elmer, Pyris, USA). After equilibration at $20^{\circ} \mathrm{C}$ for $2 \mathrm{~min}$, the sample was heated from $20^{\circ} \mathrm{C}$ to $100^{\circ} \mathrm{C}$ at a heating rate of $10^{\circ} \mathrm{C} / \mathrm{min}$, held at $100{ }^{\circ} \mathrm{C}$ for $1 \mathrm{~min}$, and cooled from $100^{\circ} \mathrm{C}$ to $20^{\circ} \mathrm{C}$ at a heating rate of $10^{\circ} \mathrm{C} / \mathrm{min}$. After equilibrating at $20^{\circ} \mathrm{C}$ for $2 \mathrm{~min}$, the sample was again heated from $20^{\circ} \mathrm{C}$ to $100^{\circ} \mathrm{C}$ at a heating rate of $10^{\circ} \mathrm{C} / \mathrm{min}$. The measurements were performed in duplicate. Data analysis was performed using the Pyris software (Perkin Elmer).

\subsubsection{Confocal laser scanning microscopy (CLSM)}

CLSM images were taken on a Zeiss LSM 510 Meta microscope (Zeiss, Germany), equipped with an Axiovert $200 \mathrm{M}$ inverted microscope (Wetzlar, Germany). About $5 \mu \mathrm{l}$ of $0.05 \%$ Calcofluor White and $5 \mu \mathrm{l}$ of $0,002 \%$ Rhodamine B were added to stain the BC microfibrils and WPI in a $1 \mathrm{ml}$ sample, respectively. After mixing, about $20 \mu \mathrm{l}$ of sample was placed in a chamber prepared from a microscopic glass slide and a cover slip. Rhodamine B was excited at a wavelength of $543 \mathrm{~nm}$ and Calcofluor white was excited at $405 \mathrm{~nm}$. The emission wavelength of Rhodamine B and Calcofluor White are $560 \mathrm{~nm}$ and $420 \mathrm{~nm}$, 
respectively. Images were taken using an oil immersion objective (EC Plan-Neoufluar $40 \times$ /1.30 Oil Dic).

\subsubsection{Transmission electron microscopy (TEM)}

TEM images were taken using a transmission electron microscope (Tecnai 20 system from FEI). Samples were diluted ten-fold using MilliQ water prior to imaging. A drop of sample was placed on a carbon copper TEM grid. The grid was pre-treated by glow discharge to make the surface hydrophilic prior to sample preparation. Excess solvent was dried by a filter paper after $15 \mathrm{~s}$. The sample was then negatively stained by adding a drop of $2 \%$ phosphortungsten acid onto the grid. The excess water was dried by another filter paper and the sample was then left air dried before imaging.

\subsubsection{Scanning electron microscopy (SEM)}

A droplet of the sample was placed on nuclepore polycarbonate membrane $(1 \mathrm{~mm}$ holes, Costar) which was placed on a filter paper to absorb the liquid through the membrane. The samples were left on the top side and air-dried. Afterwards, the membrane were glued on sample holders by carbon adhesive tabs (EMS, Washington, USA) and sputter coated with $10 \mathrm{~nm}$ Iridium (Leica EM SCD 500, Vienna, Austria). Images were taken using a high-resolution scanning electron microscope (Magellan 400, FEI, Eindhoven, the Netherlands).

Cryo-SEM was performed by placing a small droplet of the sample on a hollow copper rivet and rapidly frozen in liquid ethane. The rivets were placed in a cryo-sample holder in liquid nitrogen and afterwards transferred to the cryo-preparation system (MED 020/ VCT 100, Leica, Vienna, Austria) onto the sample stage. Afterwards, the samples were fractured, freeze dried for $10 \mathrm{~min}$ and then sputter coated with a layer of $10 \mathrm{~nm}$ tungsten at the same environmental conditions. The samples were cryo-shielded and transferred into the field emission scanning microscope (Magellan 400, FEl, Eindhoven, The Netherlands) onto the sample stage for imaging. 


\subsection{Results and discussion}

\subsubsection{Characterization of $B C$ microfibrils}

The microstructure of $B C$ microfibrils prepared from Nata de Coco by high energy mechanical de-agglomeration was characterized using TEM (Figure 1) and SEM (Figure 2).

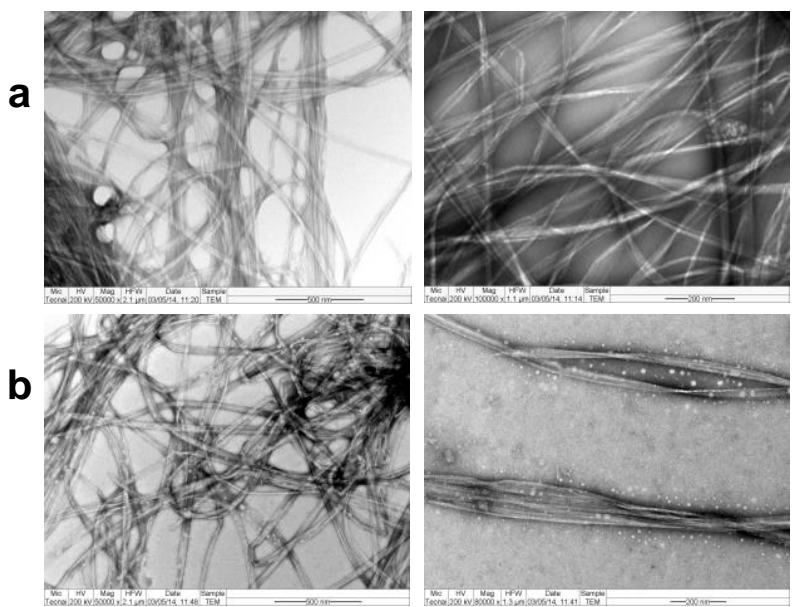

Figure 1 TEM images of 0.25 wt\% BC microfibrils before (a) and after (b) being heated at $80^{\circ} \mathrm{C}$ for $30 \mathrm{~min}$. Sample was diluted ten-fold prior to imaging. Scale bar corresponds to $500 \mathrm{~nm}$ (left) and $200 \mathrm{~nm}$ (right).

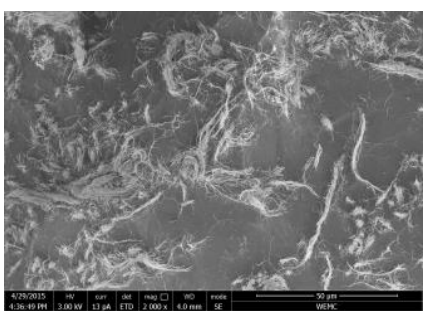

a

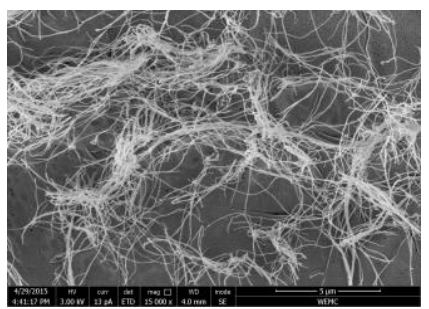

b

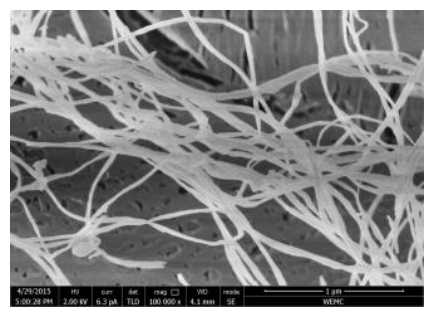

C

Figure 2 SEM images of $0.2 \mathrm{wt} \% \mathrm{BC}$ microfibrils after being heated for $30 \mathrm{~min}$ at $80^{\circ} \mathrm{C}$. Sample was diluted two-fold prior to imaging. Scale bar corresponds to $50 \mu \mathrm{m}$ (a), $5 \mu \mathrm{m}$ (b), and $1 \mu \mathrm{m}$ (c).

The TEM images (Figure 1) show the ribbon-shape structure of the BC microfibrils. The ribbon-shaped $\mathrm{BC}$ microfibrils are present in the form of bundles composed of elementary 
fibrils as induced by hydrogen bonding. Previous work reported a diameter of the elementary fibrils of about $3 \mathrm{~nm} .{ }^{38,49}$ After being heated at $80^{\circ} \mathrm{C}$ for $30 \mathrm{~min}$ at $\mathrm{pH} 7$, the microstructure of the BC microfibrils (Figure 1b), as obtained from TEM images, did not show any difference in comparison to the $B C$ microfibrils before heating (Figure 1a). The SEM images of $\mathrm{BC}$ microfibrils after being heated for $30 \mathrm{~min}$ at $80^{\circ} \mathrm{C}$ (Figure 2) showed a similar structure to the unheated $\mathrm{BC}$ microfibrils as reported in literature, ${ }^{38}$ indicating that heating did not influence the microstructure of $B C$ microfibrils. The images show the heterogeneity of the flexible and micrometer long BC microfibrils which tend to form as bundles. It has been reported that the attraction between the $\mathrm{BC}$ microfibrils are too strong to be de-agglomerated completely by high-energy mechanical de-agglomeration, and as a result, the $\mathrm{BC}$ microfibril dispersion remains heterogeneous. ${ }^{38} \mathrm{~A}$ detailed characterization of $\mathrm{BC}$ microfibrils prepared using the same method can be found elsewhere. $^{38}$

\subsubsection{Macroscopic stability}

Macroscopic stability of the WPI-BC microfibril mixtures at $4{ }^{\circ} \mathrm{C}$ was monitored over time by taking images at time 0,1 day and 7 days. Figure 3 shows the native WPI-BC microfibril dispersions, Figure 4 shows the WPI-BC microfibril dispersions after being heated at $80^{\circ} \mathrm{C}$ for 30 min and Figure 5 shows the mixtures prepared by mixing $\mathrm{BC}$ microfibrils both before $(\mathrm{BH})$ heating and after heating $(\mathrm{AH})$ the WPI solution. 


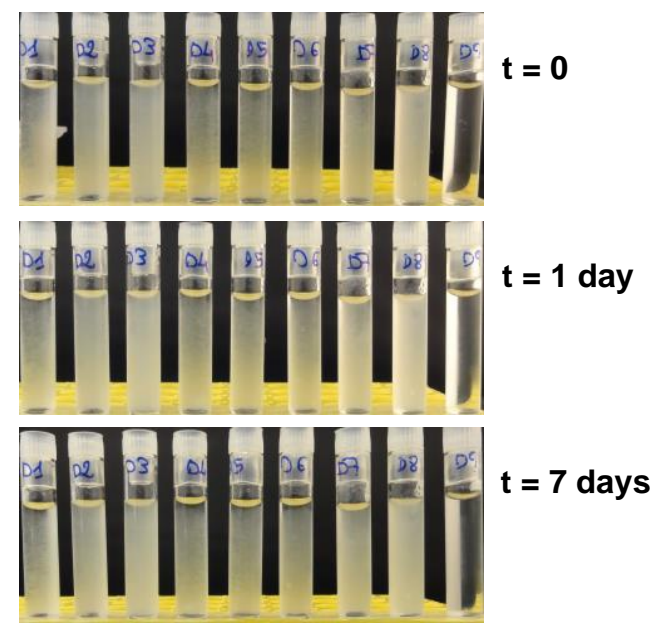

Figure 3 Images of native WPI-BC microfibril mixtures at 0,1 day and 7 days after preparation. The concentrations (in wt\%) are: $[\mathrm{WPI}, \mathrm{BC}]=[0,0.1],[0,0.2],[0,0.3],[3,0.1],[6,0.1],[9,0.1],[9,0.2]$, $[9,0.3],[9,0]$ corresponding to tubes from left to right.

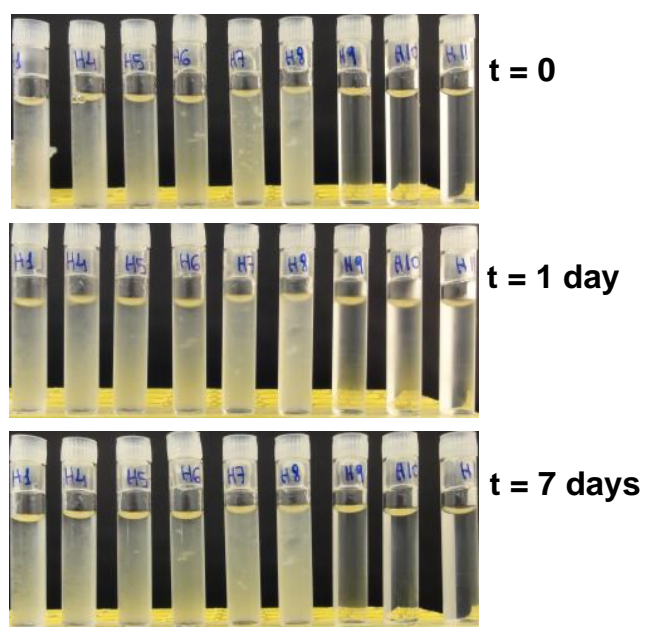

Figure 4 Images of WPI-BC microfibril mixtures after being heated at time 0,1 day and 7 days after preparation. The concentrations (in wt\%) are: $[\mathrm{WPI}, \mathrm{BC}]=[0,0.1],[3,0.1],[6,0.1],[9,0.1],[9,0.2]$, $[9,0.3],[3,0],[6,0],[9,0]$ corresponding to tubes from left to right. 


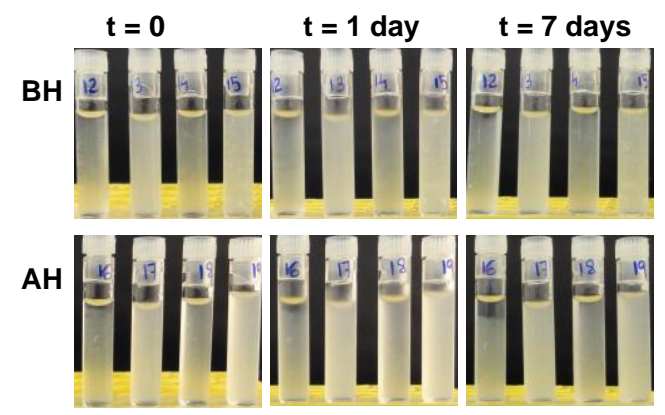

Figure 5 Images of WPI-BC microfibril mixtures prepared by mixing BC microfibrils both before heating $(\mathrm{BH})$ and after heating $(\mathrm{AH})$ the WPI solution at time 0,1 day and 7 days after sample preparation. Concentrations (in wt\%) are: $[\mathrm{WPI}, \mathrm{BC}]=[3,0.1],[3,0.3],[4.5,0.1],[4.5,0.3](\mathrm{BH})$ and $[W P I, B C]=[3,0.1],[3,0.3],[4.5,0.1],[4.5,0.3](A H)$ from left to right.

In the native (i.e. unheated) mixtures (Figure 3) containing $0.1 \mathrm{wt} \% \mathrm{BC}$ microfibrils, some sedimentation of the BC microfibrils was observed after 7 days. Adding WPI up to $9 \mathrm{wt} \%$ into the mixtures did not show any difference from the dispersion containing only $B C$ microfibrils. When BC microfibril concentration is $0.2 \mathrm{wt} \%$ and above, samples remained stable macroscopically for at least 7 days after sample preparation. Adding WPI to the dispersions leads to the same macroscopic stability as those of BC microfibril dispersions without WPI. This can be attributed to the space-filling network formed by $B C$ microfibrils. ${ }^{38}$ Previous research has shown that the network of BC microfibrils with increasing $B C$ microfibril concentration evolves from forming a collapsed gel to a selfsupporting gel. ${ }^{38}$ The macroscopic behavior indicates that WPI and BC microfibrils form stable mixtures under the conditions studied.

Upon heating the samples for $30 \mathrm{~min}$ at $80^{\circ} \mathrm{C}$, the macroscopic stability of the dispersions (Figure 4) is comparable to that of the native WPI-BC microfibril dispersions. Interestingly, a few large flocs were observed in the WPI-BC microfibril dispersions containing $9 \mathrm{wt} \%$ WPI, whereas this was not observed in pure BC microfibril dispersions. This suggests that the large flocs consist of WPI, although no further analysis was performed on these flocs. The macroscopic stability of the WPI-BC microfibril mixtures after heating suggests that WPI and BC microfibrils form stable mixtures upon heating.

To investigate whether BC microfibrils and WPI denaturation and aggregation mutually influence one another during heating, we compared heated WPI-BC microfibril dispersions 
with WPI-BC microfibril dispersions that were obtained by mixing heated WPI dispersions with heated BC microfibril dispersions. We refer to these two types of dispersions as WPI$B C$ microfibril mixtures prepared by mixing $B C$ microfibrils before heating $(\mathrm{BH})$ and after heating $(\mathrm{AH})$ the WPI solution. As shown in Figure 5, in samples containing $3 \mathrm{wt} \% \mathrm{WPI}$ and 0.1 wt\% BC microfibrils, phase separation was observed. The AH mixture of [WPI, BC] = [3, 0.1] showed more phase separation than the $\mathrm{BH}$ mixture. This phase separation became less for $[\mathrm{WPI}, \mathrm{BC}]=[4.5,0.1]$, i.e. higher WPI concentration, possibly due to the viscosity increase of the continuous phase by the higher protein concentration. In both $\mathrm{BH}$ and $\mathrm{AH}$ dispersions containing $0.2 \mathrm{wt} \% \mathrm{BC}$ microfibrils, all samples remained stable. Apparently, 0.2 wt\% BC microfibrils or higher prevents phase separation, concomitant with the stability of dispersions of BC microfibrils alone that contain more than $0.2 \mathrm{wt} \% \mathrm{BC}$ microfibrils (Figure 3). We conclude that BC microfibrils and WPI denaturation and aggregation do not macroscopically influence one another during heating.

\subsubsection{DSC}

To investigate whether the presence of BC microfibrils affects the WPI denaturation upon heating, DSC thermogram was recorded (Figure 6).

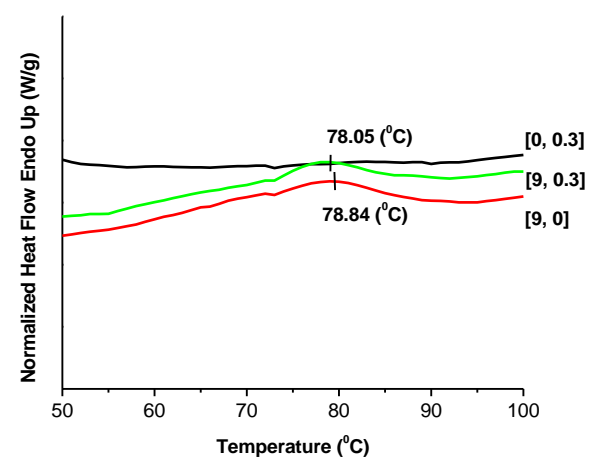

Figure 6 DSC thermograms of WPI-BC microfibril dispersions with $[\mathrm{WPI}, \mathrm{BC}]=[0,0.3],[9,0.3],[9,0]$. Concentrations (wt\%) are indicated below the curve.

The pure BC microfibril dispersion does not show a peak upon heating (cf. Figure 6). The pure WPI solution give an endothermic peak upon heating, which refers to the 
denaturation of the WPI. ${ }^{47}$ The peak denaturation temperature is around $79^{\circ} \mathrm{C}$, which is in line with literature. ${ }^{44,50}$ Adding 0.3 wt\% BC microfibrils into $9 \mathrm{wt} \% \mathrm{WPI}$, an endothermic peak with a peak denaturation temperature of $78{ }^{\circ} \mathrm{C}$ was also observed. This suggests that the WPI denaturation temperature is not affected by the presence of $B C$ microfibrils. This serves as an indication of the absence of specific interactions between WPI and BC during heating. ${ }^{51}$

\subsubsection{Rheological properties}

The viscosity of the WPI-BC microfibril dispersions is measured by first increasing the shear rate from 0.1 to 500 1/s, then decreasing the shear rate from 500 to 0.1 1/s. It is known that both sample preparation and the shear history of the sample affect the rheological properties of the $\mathrm{BC}$ microfibril dispersions. ${ }^{41}$ To minimize this influence, a time sweep was performed prior to the viscosity measurement.

\subsubsection{Native WPI-BC microfibril dispersions}
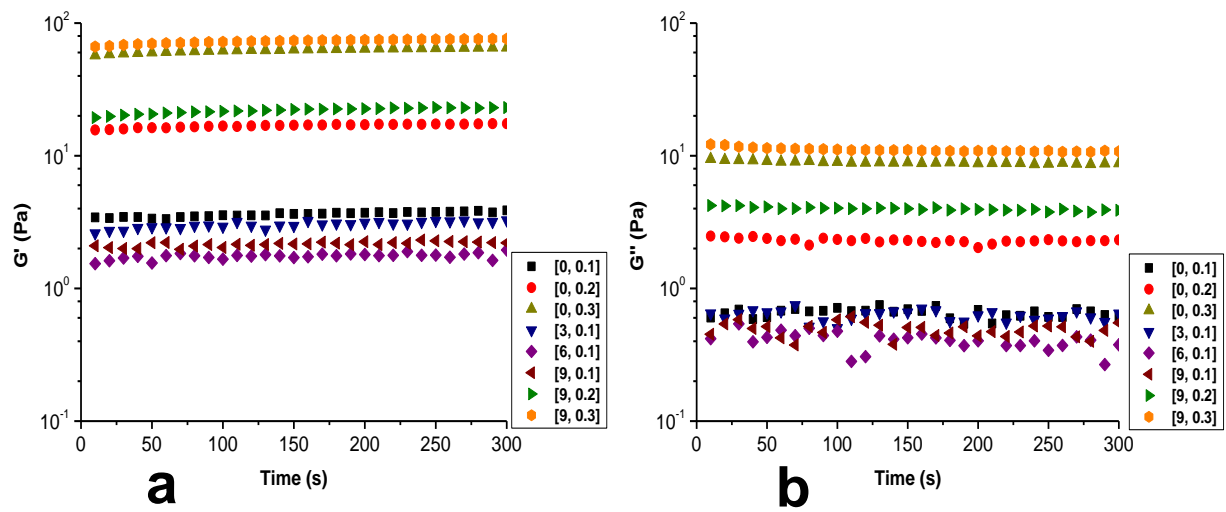

Figure 7 Storage modulus G' (a) and loss modulus G" (b) of native (unheated) WPI-BC microfibril dispersions as a function of time at a frequency of $1 \mathrm{~Hz}$ and strain of $0.1 \%$. Concentrations of WPI $(w t \%)$ and $B C(w t \%)$ are given by $[\mathrm{WPI}, \mathrm{BC}]=[0,0.1],[0,0.2],[0,0.3],[3,0.1],[6,0.1],[9,0.1],[9$, $0.2],[9,0.3]$. 

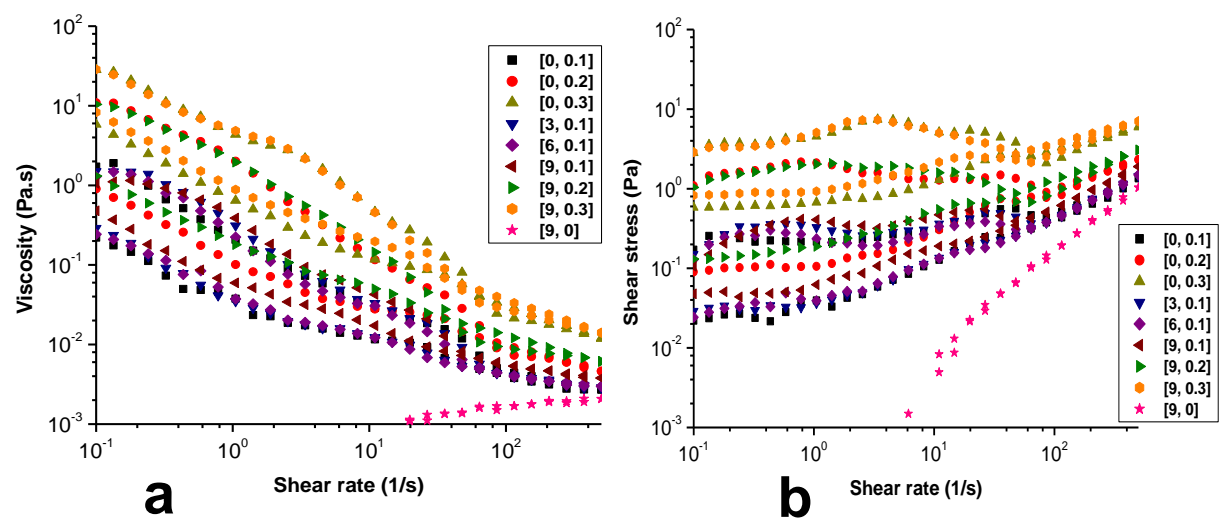

Figure 8 Viscosity (a) and shear stress (b) of native (unheated) WPI-BC microfibril dispersions as a function of shear rate. Shear rate was reversed after increasing from 0.1 to $5001 / \mathrm{s}$. Concentrations of WPI ( wt\%) and BC microfibrils (wt\%) are given by $[\mathrm{WPI}, \mathrm{BC}]=[0,0.1],[0,0.2]$, $[0,0.3],[3,0.1],[6,0.1],[9,0.1],[9,0.2],[9,0.3],[9,0]$.

During the pre-shear period, the $\mathrm{G}^{\prime}$ and $\mathrm{G}^{\prime \prime}$ of pure $\mathrm{BC}$ microfibril dispersions changed slowly over time (Figure 7). The $G^{\prime}$ gradually increased and G" gradually decreased as a function of time. The extent of increase of $G^{\prime}$ and decrease of $G^{\prime \prime}$ is enhanced for increasing BC microfibril concentration from 0.1 to $0.3 \mathrm{wt} \%$. In the mixtures of WPI-BC microfibrils, the change of $G^{\prime}$ and $G^{\prime \prime}$ over time are comparable to those of the according pure $B C$ microfibril dispersions. The values of $G^{\prime}$ and $G^{\prime \prime}$ in the mixtures are close to the values in the pure BC microfibril dispersions, suggesting that the $G^{\prime}$ and $G^{\prime \prime}$ are mainly controlled by the presence of BC microfibrils. The $\mathrm{G}^{\prime}$ and $\mathrm{G}^{\prime \prime}$ of pure WPI solutions (3-9 $w t \%)$ were too low to be measured. The change of $G^{\prime}$ and $G^{\prime \prime}$ over time has also been reported in the mixtures of $\mathrm{BC}$ and $\mathrm{CMC}^{41}$ and microfibrillated cellulose from plant cellulose. $^{33,52,53}$ The reason of this change was proposed to reside in the slow reformation of contacts between the microfibrils after the disturbance by the loading of the measurement cup. Indeed, one should take the history of the sample and time into consideration for comparison. ${ }^{41}$

The viscosity of pure BC microfibril dispersions shows strong shear thinning behaviour and thixotropic behaviour (Figure 8a), in agreement with literature. ${ }^{38,41}$ Interestingly, a viscosity plateau was observed in between two shear thinning regions, which was also 
reported previously. ${ }^{54}$ This can also be concluded from the slope of the shear stress versus shear rate curve as shown in Figure $8 \mathrm{~b}$. The viscosity of the dispersions increases with increase in BC concentrations. The typical viscosity plateau and its dependency on concentration have also been reported for microfibrillated cellulose from plant cellulose. ${ }^{31,}$ $32,52,53,55-58$ The reason for the viscosity plateau was given in terms of a change of the network structure during shearing. ${ }^{52,56}$ At the shear rate where the viscosity plateau starts to occur, a significant increase in floc size and its distribution was observed, as well as fibril-free voids. Above a sufficiently high shear rate, these flocs are broken into smaller sizes and the system becomes shear thinning again. ${ }^{52,56}$ The inhomogeneously distributed flocs with different sizes have been attributed to a shear banding phenomenon resulting in different flow regimes within one sample. ${ }^{33,38,55}$ The above findings have also been reported in hydrolysed and TEMPO-oxidized microfibrillated/nanofibrillated cellulose suspensions. ${ }^{57}$ The above is also in accordance with the results in Figure 8 . The increase of viscosity with increase in $\mathrm{BC}$ microfibril concentration is in agreement with literature, and is attributed to more crosslinks per unit volume, i.e. a denser network. ${ }^{38,41}$

In the WPI-BC microfibril mixtures, the viscosity of the mixtures (Figure 8a) is comparable to the corresponding $B C$ microfibril dispersions for equal $B C$ microfibril concentration. The shear stress versus shear rate curves of the mixtures also followed the same trend as that of the pure $\mathrm{BC}$ microfibril dispersions (Figure $8 \mathrm{~b}$ ). This indicates that the rheological behaviour of the samples is mainly controlled by the BC microfibrils and almost not affected by the presence of WPI.

A joint inspection of viscosity over shear rate increase and followed decrease convey socalled hysteresis for all samples containing BC, i.e. the value of viscosity is different from the initial value when the shear is reversed. This is similar to that reported for microfibrillated cellulose of plant based origin. ${ }^{53}$ The viscosity plateau occurring for decreasing shear rate is less pronounced than that occurring for increasing shear rate (Figure 8). It was apparent that the viscosity of the mixtures is dominated by the presence of $\mathrm{BC}$ microfibrils in the mixtures (Figure $8 \mathrm{a}$ ). Similar hysteresis findings have also been reported for a microfibrillated cellulose/polymer system. ${ }^{33}$ 


\subsubsection{Heated WPI-BC microfibril dispersions}
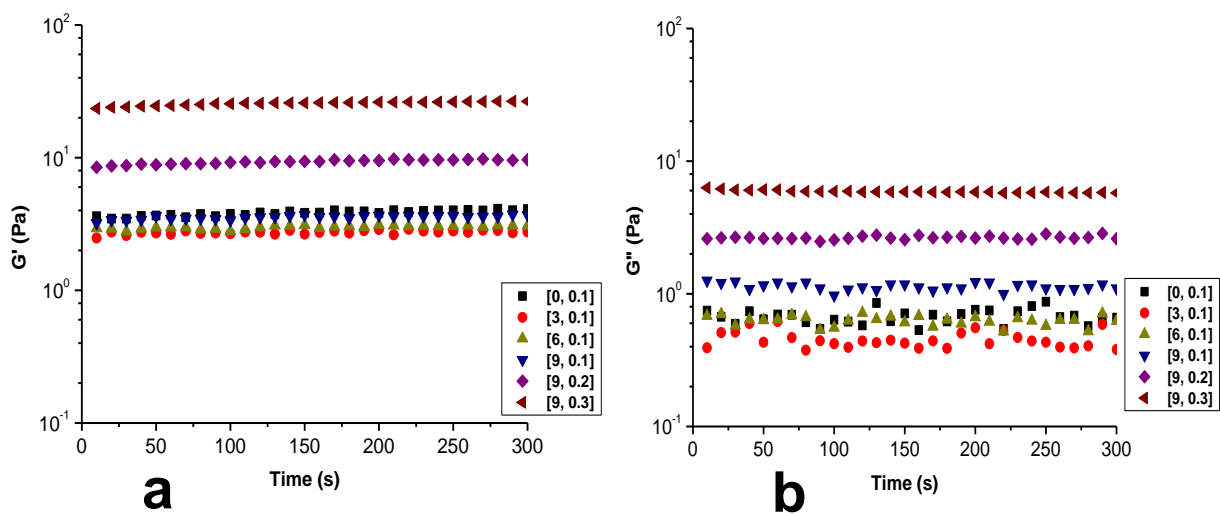

Figure 9 Storage modulus G' (a) and loss modulus G' (b) of heated WPI-BC microfibril dispersions as a function of time at a frequency of $1 \mathrm{~Hz}$ and strain of $0.1 \%$. Concentrations of WPI ( wt\%) and $B C$ microfibrils $(w t \%)$ are $[W P I, B C]=[0,0.1],[3,0.1],[6,0.1],[9,0.1],[9,0.2],[9,0.3]$.
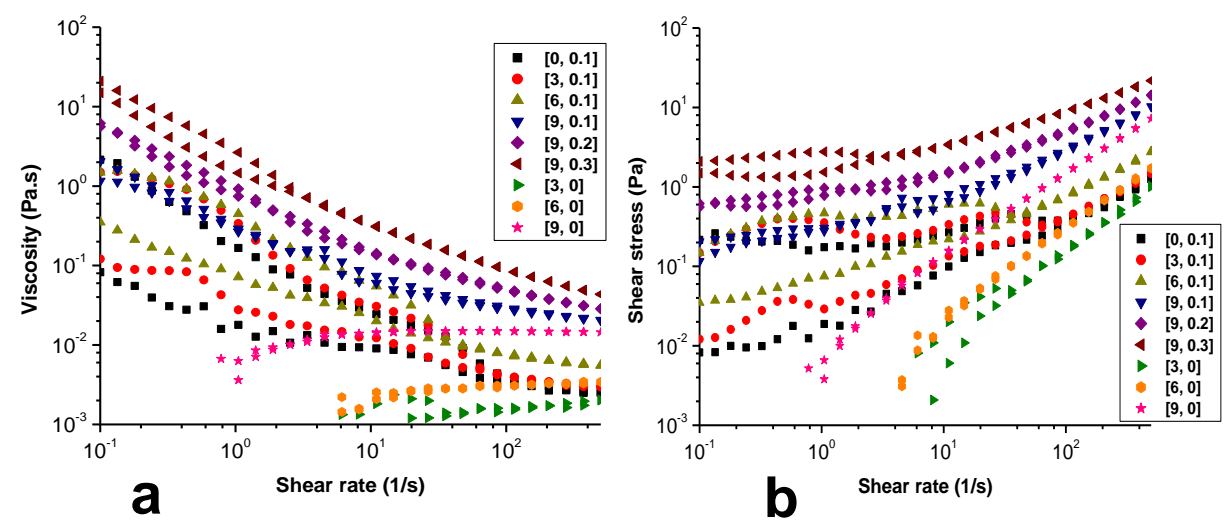

Figure 10 Viscosity (a) and shear stress (b) of heated WPI-BC microfibril dispersions as a function of shear rate. Shear rate was reversed after increasing from 0.1 to $5001 / \mathrm{s}$. Concentrations of WPI $(w t \%)$ and BC microfibrils (wt\%) are $[\mathrm{WPI}, \mathrm{BC}]=[0,0.1],[3,0.1],[6,0.1],[9,0.1],[9,0.2],[9,0.3]$, $[3,0],[6,0],[9,0]$. 
After heating, the $G^{\prime}$ and $G^{\prime \prime}$ of the 0.1 wt\% pure BC microfibril dispersions (Figure 9) remained equal to that before heating. The change of $G^{\prime}$ and $G^{\prime \prime}$ over time and its increase with increasing $\mathrm{BC}$ microfibril concentration was comparable to that of the native mixtures (Figure 7). The $G^{\prime}$ and $G^{\prime \prime}$ of the WPI-BC microfibril mixtures containing 0.1 wt\% BC microfibrils was similar to that of the $0.1 \mathrm{wt} \%$ pure BC microfibril dispersion after heating. In the case of mixtures containing 0.2 and $0.3 \mathrm{wt} \% \mathrm{BC}$ microfibrils, the $\mathrm{G}^{\prime}$ and $\mathrm{G}^{\prime \prime}$ slightly decreased after heating in comparison to native mixtures in Figure 7.

The viscosity of the samples after heating, including the pure BC microfibrils (Figure 10) showed strong shear thinning behaviour, and had values similar to those of the native samples (Figure 8a). This indicates that the BC microfibril network and BC microfibrils themselves are stable upon heating, in line with literature. ${ }^{59-61}$ The temperature independency has also been found in microfibrillated cellulose from plant-based origin. ${ }^{31}$ 32, 55 The hysteresis, i.e. lower viscosity when shear rate is reversed, is still observed in pure $0.1 \mathrm{wt} \% \mathrm{BC}$ microfibril dispersion and dispersions containing both 0.1 wt\% BC and WPI from 3 to 6 wt\%. However, in mixtures containing 9 wt\% WPI, the hysteresis is absent in comparison to the native mixtures where hysteresis is observed. Heating the mixtures at $\mathrm{pH} 7$ for $30 \mathrm{~min}$ at $80^{\circ} \mathrm{C}$ does not influence significantly the viscosity of the mixtures, i.e. $\mathrm{BC}$ microfibrils are the dominant factor for the viscosity of the mixtures after heating.

\subsubsection{Mixing $B C$ microfibrils before $(\mathrm{BH})$ and after heating $(\mathrm{AH})$ the WPI solution}

It is known that heating WPI alone at $\mathrm{pH} 7$ at $80^{\circ} \mathrm{C}$ for 30 min results in the denaturation and aggregation of the WPI. ${ }^{43,50}$ To investigate whether the presence of $\mathrm{BC}$ microfibrils affects the denaturation and aggregation of WPI upon heating, the viscosity of the mixtures prepared by both mixing BC microfibrils before and after heating the WPI solution was measured. The $G^{\prime}$ and $G^{\prime \prime}$ over time, viscosity and shear stress as a function of shear rate are shown in Figures 11-12. 

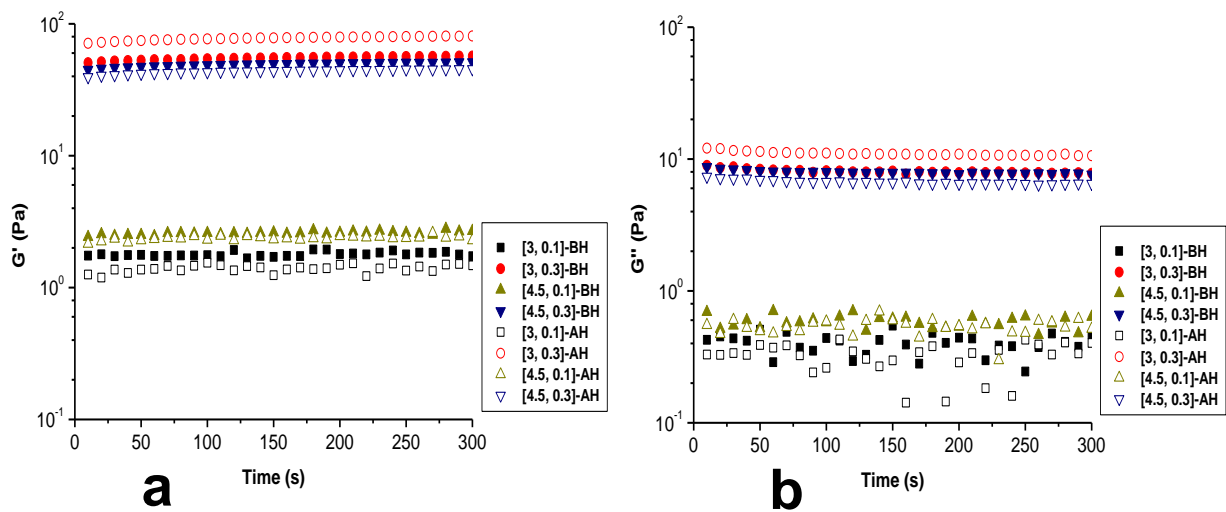

Figure 11 Storage modulus G' (a) and loss modulus G' (b) over time of WPI-BC dispersions prepared by mixing $\mathrm{BC}$ micrfibrils before $(\mathrm{BH})$ and after heating $(\mathrm{AH})$ the WPI solution at a frequency of $1 \mathrm{~Hz}$ and strain of $0.1 \%$. Concentrations of WPI ( wt\%) and BC microfibrils (wt\%) are $[W P I, B C]=[3,0.1],[3,0.3],[4.5,0.1],[4.5,0.3]$. Filled symbols refer to $B H$ sampples and open symbols refer to $\mathrm{AH}$ samples.
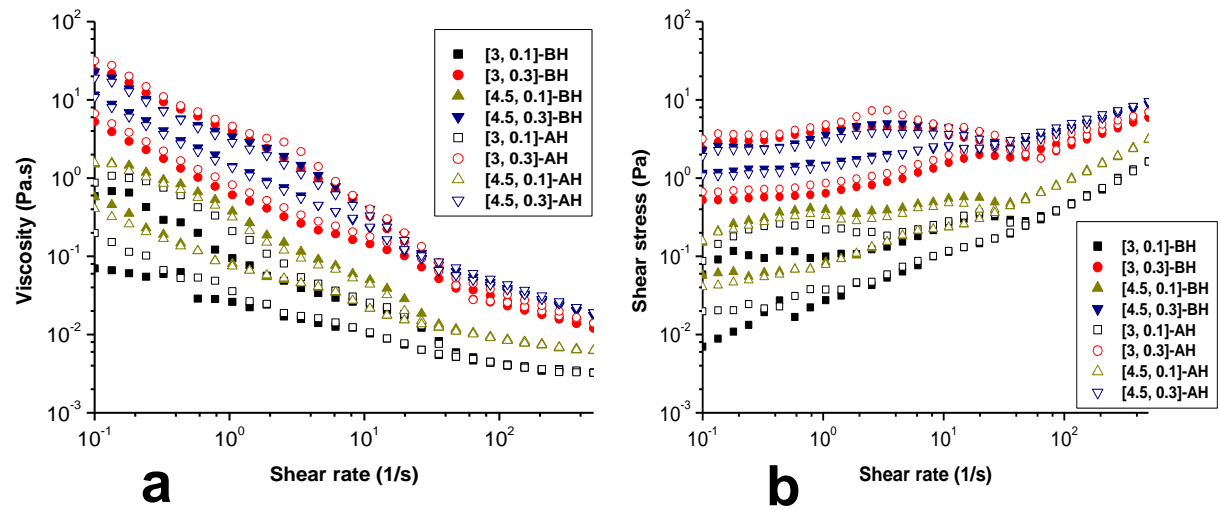

Figure 12 Viscosity (a) and shear stress (b) as a function of shear rate of WPI-BC dispersions prepared by mixing $B C$ microfibrils before $(\mathrm{BH})$ and after heating $(\mathrm{AH})$ the WPI solution. Shear rate was reversed after increasing from 0.1 to 500 1/s. Concentrations of WPI (wt\%) and BC microfibrils (wt\%) are given by $[\mathrm{WPI}, \mathrm{BC}]=[3,0.1],[3,0.3],[4.5,0.1],[4.5,0.3]$. Filled symbols refer to $\mathrm{BH}$ samples and open symbols refer to AH samples. 
As can be seen in Figure 11, the $\mathrm{G}^{\prime}$ and $\mathrm{G}^{\prime \prime}$ over time of all mixtures showed a similar trend to the native and heated mixtures (cf. Figure 7 and 9). The $\mathrm{G}^{\prime}$ and $\mathrm{G}^{\prime \prime}$ of $\mathrm{BH}$ mixtures did not show a clear difference from AH mixtures. The viscosity (cf. Figure 12a) and shear stress (cf. Figure 12b) as a function of shear rate showed the same trends for both $\mathrm{BH}$ and AH samples. Again, BC microfibrils turn out to be the dominant factor for viscosity of the heated mixtures. In other words, the presence of BC microfibrils apparently did not significantly influence the denaturation (and subsequent aggregation) of WPI during heating. The former is in agreement with our DSC results. It was noted that the hysteresis of the flow curves after reversing the shear are still present in those mixtures.

\subsubsection{Size distribution of WPI aggregates}

The size distribution of the WPI aggregates formed upon heating in the mixtures was measured to investigate whether the presence of $\mathrm{BC}$ microfibrils influences the aggregate size of the WPI. The size of the WPI aggregates in BH mixtures were compared to those of the $\mathrm{AH}$ mixtures. In order to avoid interference of the BC microfibrils in the measurements, the mixtures were centrifuged and the WPI aggregates in the supernatant were taken for analysis.

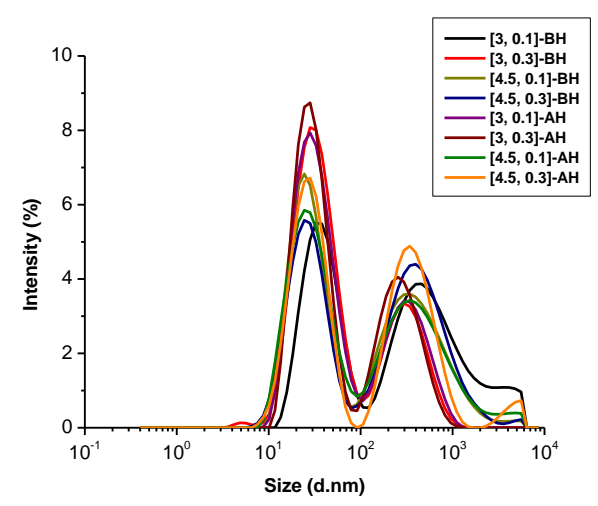

Figure 13 The size distribution of WPI aggregates after centrifugation of WPI-BC microfibril dispersions prepared by mixing $\mathrm{BC}$ micrfibrils before $(\mathrm{BH})$ and after heating $(\mathrm{AH})$ the WPI solution. Concentrations of WPI (wt\%) and BC microfibrils (wt\%) are given by $[\mathrm{WPI}, \mathrm{BC}]=[3,0.1],[3,0.3]$, $[4.5,0.1],[4.5,0.3]$. 
The size distribution of WPI aggregates in mixtures of both $\mathrm{BH}$ and $\mathrm{AH}$ were similar, with two peaks observed (Figure 13). The average WPI aggregate sizes formed in the presence of $B C$ microfibrils $(\mathrm{BH})$ was comparable to the WPI aggregate sizes formed alone $(A H)$. Although the intensity varies between the samples, no clear difference can be concluded. This implies that the denaturation and aggregation of WPI is not affected by the presence of $B C$ microfibrils, which is in agreement with the DSC and viscosity results. It is noted that the aggregate sizes we measured are the WPI aggregates from the supernatant after centrifugation.

\subsubsection{Confocal laser scanning microscopy (CLSM)}

In native BC dispersions, the microfibrils are visible using CSLM in the form of flocs of microfibrils (Figure 14) in accordance with literature. ${ }^{38}$ The native WPI dispersion shows a homogeneous structure, since the molecules are too small to be visualized under this magnification. In the native WPI-BC microfibril mixtures, it was observed that both WPI and $\mathrm{BC}$ microfibrils independently present in the overlay images. The microstructures of $B C$ microfibrils shown in the mixtures are identical with those in the pure $B C$ microfibril dispersion, and are independent of WPI concentration. The WPI remained homogeneous for all $\mathrm{BC}$ microfibril concentrations. After heating at $80^{\circ} \mathrm{C}$ for $30 \mathrm{~min}$, the microstructure of all samples remained the same as that of the native mixtures. WPI forms aggregates upon heating, however, because the size of the aggregates are too small to be visualized, a homogeneous WPI system was observed. The BC microfibrils remained heterogeneous in the mixtures, consisting of flocs and voids. The adsorption of WPI on the surface of BC microfibrils cannot be confirmed from the CLSM images. The WPI network and BC microfibril network seemed to be independently present in one system, and no aggregation could be observed. 

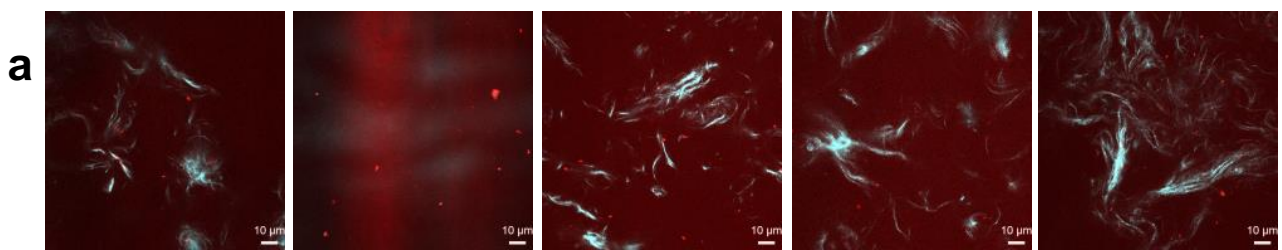

b
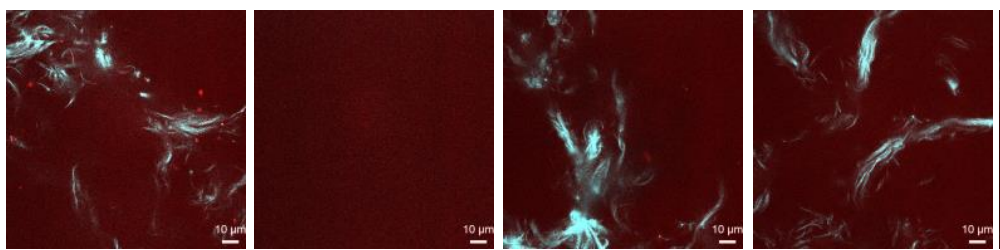

$[0,0.1]$

$[9,0]$

$[3,0.1]$

$[9,0.1]$

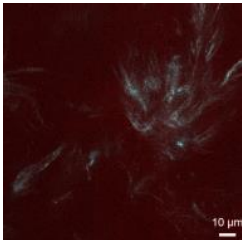

$[9,0.3]$

Figure 14 CLSM images of native (a) and heated (b) WPI-BC microfibril dispersions. Concentrations of WPI ( wt\%) and BC microfibrils (wt\%) are given by $[\mathrm{WPI}, \mathrm{BC}]=[0,0.1],[9,0],[3,0.1],[9,0.1],[9$, 0.3]. WPI is indicated in red and BC is indicated in turquoise. Scale bar $=10 \mu \mathrm{m}$.

Comparing the CLSM images of the $\mathrm{BH}$ mixtures to $\mathrm{AH}$ mixtures, as shown in Figure 15, no clear difference, for any of the concentration ratios used can be observed. Both the WPI network and the $B C$ microfibril network are present. This suggests that the presence of $B C$ microfibrils do not influence the WPI denaturation and aggregation and, vice versa, that the WPI does not influence the BC microfibril network. It is concluded that no specific interactions between WPI and BC microfibrils exist that change their respective behaviour in the mixture as compared to on its own. 


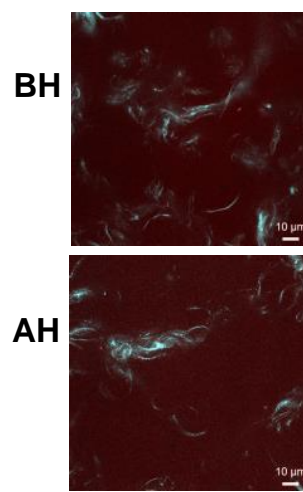

$[3,0.1]$
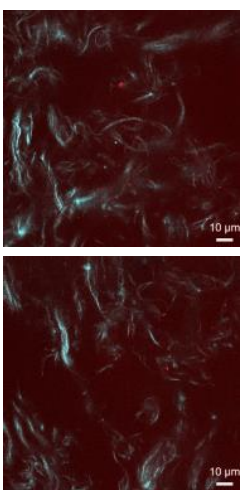

$[3,0.3]$
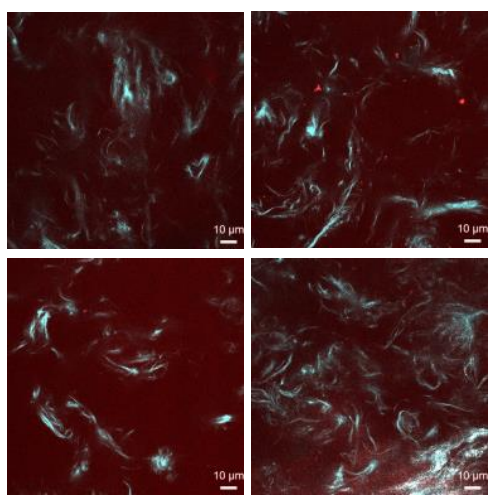

$[4.5,0.1]$

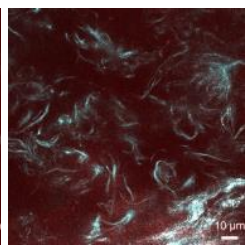

$[4.5,0.3]$

Figure 15 CLSM images of WPI-BC dispersions prepared by mixing BC micrfibrils before (BH) and after heating (AH) the WPI solution. Concentrations of WPI (wt\%) and BC microfibrils (wt\%) are given by $[\mathrm{WPI}, \mathrm{BC}]=[3,0.1],[3,0.3],[4.5,0.1],[4.5,0.3]$. WPI is indicated in red and $\mathrm{BC}$ is indicated in turquoise. Scale bar $=10 \mu \mathrm{m}$.

\subsubsection{Scanning electron microscopy (SEM)}

SEM images are taken to investigate the morphology of WPI aggregates and BC microfibrils, results are shown in Figures 16-18. 


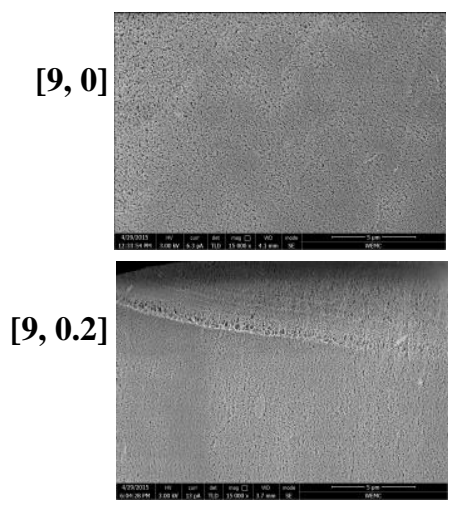

a
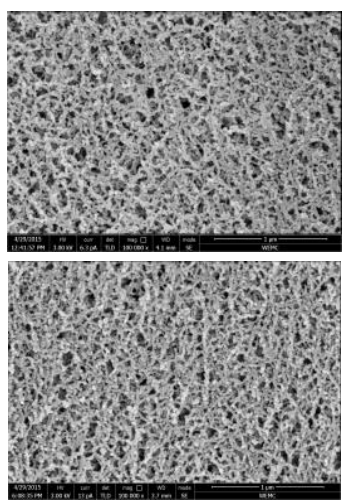

b

Figure 16 Cryo-SEM images of heated WPI-BC microfibril dispersions. Concentrations of WPI ( wt\%) and $B C$ microfibrils (wt\%) are given by $[\mathrm{WPI}, B C]=[9,0]$ and $[9,0.2]$. Scale bars $=5 \mu \mathrm{m}(\mathrm{a})$ and $1 \mu \mathrm{m}$ (b).

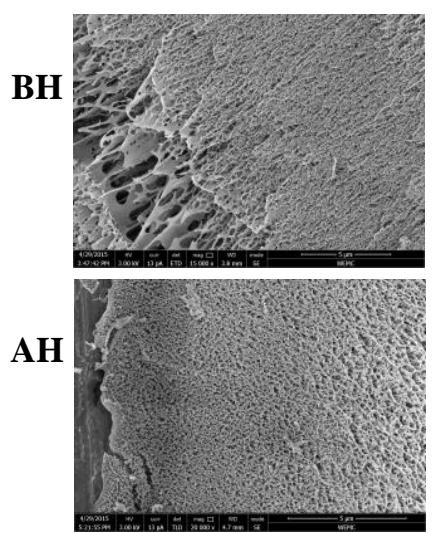

a

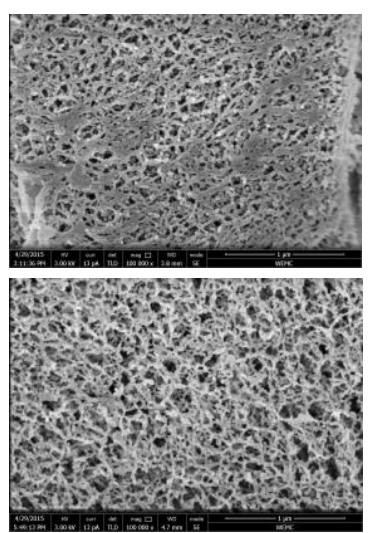

b

Figure 17 Cryo-SEM images of mixtures prepared by mixing BC micrfibrils before (BH) and after heating (AH) the WPI solution. Concentrations of WPI (wt\%) and BC microfibrils (wt\%) are given by $[W P I, B C]=[4.5,0.2]$. Scale bars $=5 \mu \mathrm{m}(\mathrm{a})$ and $1 \mu \mathrm{m}(\mathrm{b})$. 


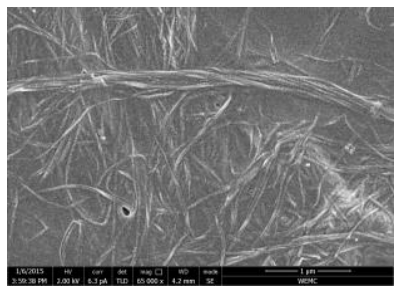

BH

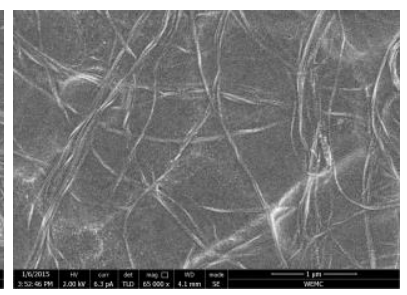

AH

Figure 18 SEM images of mixtures prepared by mixing $\mathrm{BC}$ micrfibrils before $(\mathrm{BH})$ and after heating (AH) the WPI solution. Concentrations of WPI (wt\%) and BC microfibrils (wt\%) are given by $[\mathrm{WPI}, \mathrm{BC}]=[4.5,0.15]$. Samples were air-dried on filter paper prior to imaging. Scale bar $=1 \mu \mathrm{m}$.

In Figure 16, the cryo-SEM images of $9 \mathrm{wt} \%$ pure WPI solution and mixture containing $9 \mathrm{wt} \%$ WPI and 0.2 wt\% BC microfibrils show an identical WPI network, i.e. a homogeneous WPI network consisting of WPI fine strands. This indicates that the presence of BC microfibrils did not influence the WPI aggregates formation during heating. These results are in agreement with our results of DSC, viscosity and CLSM. The WPI network of the BH and AH mixtures also did not show significant difference on the morphology of the WPI network composed of fine strands, as shown in Figure 17. In the image at the left hand side of Figure 17, a slight alignment of the protein structure may be discerned, possibly due to the heating in the presence of the $B C$ microfibrils. However, this may be only an effect locally near the BC microfibrils. The other characteristics of the WPI network structure like fine strands seem to be not affected. The overall results confirm that the WPI aggregation was not drastically affected by the presence of $B C$ microfibrils. When the mixtures were airdried prior to imaging (Figure 18) the WPI network and BC microfibril network are homogeneously and independently present in the same system. No clear difference on WPI network and $\mathrm{BC}$ microfibril network can be observed between $\mathrm{BH}$ and $\mathrm{AH}$ mixtures.

In conclusion, we have shown that mixing WPI and BC microfibrils together and subsequently heating these dispersions occur as if WPI and BC microfibrils would be present separately. DSC result showed no influence of BC microfibrils on the denaturation temperature of WPI. The viscosity of the mixtures is dominated by the presence of $\mathrm{BC}$ microfibrils. The sample showed two shear-thinning regions separated by a viscosity plateau, likely due to the flocculation of BC microfibrils. When samples are subjected to heating at $80{ }^{\circ} \mathrm{C}$ for $30 \mathrm{~min}$, the resulted mixtures remained macroscopically stable, but 
some flocs possibly formed by WPI was observed. The viscosity of the heated mixtures was comparable to that of the native mixtures and was dominated by $\mathrm{BC}$ microfibrils. The viscosity plateau was less pronounced in mixtures containing $9 \mathrm{wt} \%$ WPI after heating as compared to before heating. Mixing $B C$ micrfibrils either before $(B H)$ or after heating $(A H)$ the WPI solution prior to measurement did not show distinct results on viscosity of the mixtures. These results, together with our results on DSC, CSLM and SEM, indicate that the WPI aggregation is not affected by the presence of BC microfibrils and, vice versa, that the $\mathrm{BC}$ microfibril network structure is not influenced by the WPI aggregation.

Analogously to our results of WPI and BC microfibrils, when adding low-methoxyl pectin (LM-pectin) to microfibrillated plant cellulose, it was also found that viscoelastic and microstrutural properties of microfibrillated cellulose suspensions were not significantly modified by the presence of LM-pectin. ${ }^{33}$ We observed no influence of addition of WPI, nor its heating and subsequent aggregation, on the rheology and microstructure of $\mathrm{BC}$ microfibrils., similarly to what was observed for polymers like guar gum and xylan that did not have a significant effect on the flocculation in cellulose fibril systems. ${ }^{35}$

\subsection{Conclusion}

In mixed dispersions of WPI and BC microfibrils, the WPI aggregation and network structure are not affected by the presence of BC microfibrils and, vice versa, the BC microfibril network structure is not influenced by the WPI aggregation. The WPI and BC mixed dispersions, both before and after heating, behave as if the WPI and BC microfibrils do not experience the presence of one another.

\subsection{Acknowledgement}

This work is supported by NanoNextNL, a micro and nanotechnology consortium of the Government of the Netherlands and 130 partners. We thank Tiny Franssen-Verheijen for SEM image analysis. 


\subsection{References}

1. E. Dickinson, Food Hydrocolloids, 2003, 17, 25-39.

2. V. Y. Grinberg and V. B. Tolstoguzov, Food Hydrocolloids, 1997, 11, 145-158.

3. S. Turgeon, M. Beaulieu, C. Schmitt and C. Sanchez, Current Opinion in Colloid \& Interface Science, 2003, 8, 401-414.

4. J.-L. Doublier, C. Garnier, D. Renard and C. Sanchez, Current Opinion in Colloid \& Interface Science, 2000, 5, 202-214.

5. V. Tolstoguzov, Food Hydrocolloids, 1991, 4, 429-468.

6. A. A. Perez, C. R. Carrara, C. C. Sánchez, J. M. Rodríguez Patino and L. G. Santiago, Food Chemistry, 2009, 116, 104-113.

7. A. Benichou, A. Aserin, R. Lutz and N. Garti, Food Hydrocolloids, 2007, 21, 379-391.

8. C. M. Bryant and D. J. McClements, Food Hydrocolloids, 2000, 14, 383-390.

9. J.-Y. Chun, G.-P. Hong, S. Surassmo, J. Weiss, S.-G. Min and M.-J. Choi, Polymer, 2014, 55, 43794384.

10. K. G. de Kruif and R. Tuinier, International Journal of Food Science \& Technology, 1999, 34, 487492.

11. Y. Hemar, M. Tamehana, P. A. Munro and H. Singh, Food Hydrocolloids, 2001, 15, 565-574.

12. R. Lutz, A. Aserin, Y. Portnoy, M. Gottlieb and N. Garti, Colloids and Surfaces B: Biointerfaces, 2009, 69, 43-50.

13. N. Neirynck, P. Van der Meeren, M. Lukaszewicz-Lausecker, J. Cocquyt, D. Verbeken and K. Dewettinck, Colloids and Surfaces A: Physicochemical and Engineering Aspects, 2007, 298, 99107.

14. C. Tavares and J. A. Lopes Da Silva, International dairy journal, 2003, 13, 699-706.

15. R. Tuinier, J. K. G. Dhont and C. G. De Kruif, Langmuir, 2000, 16, 1497-1507.

16. F. Weinbreck, H. Nieuwenhuijse, G. W. Robijn and C. G. De Kruif, Journal of Agricultural and Food Chemistry, 2004, 52, 3550-3555.

17. F. Weinbrreck, R. H. Tromp and C. G. de Kruif, Biomacromolecules, 2004, 5, 1437-1445.

18. C. G. De Kruif and R. Tuinier, Food Hydrocolloids, 2001, 15, 555-563.

19. C. G. de Kruif, F. Weinbreck and R. de Vries, Current Opinion in Colloid \& Interface Science, 2004, 9, 340-349.

20. S. K. Samant, R. S. Singhal, P. R. Kulkarni and D. V. Rege, International Journal of Food Science \& Technology, 1993, 28, 547-562.

21. V. Tolstoguzov, Functional properties of food macromolecules, 1998, 252.

22. V. Tolstoguzov, D. E. B. Harry Walter and A. S. Paul, in International Review of Cytology, Academic Press, 1999, vol. Volume 192, pp. 3-31.

23. H. Firoozmand and D. Rousseau, Food Hydrocolloids, 2015, 50, 84-93. 
24. K. K. T. Goh, A. Sarkar and H. Singh, in Milk Proteins, 2008, pp. 347-376.

25. D. J. McClements, Biotechnology Advances, 2006, 24, 621-625.

26. S. L. Turgeon, C. Schmitt and C. Sanchez, Current Opinion in Colloid \& Interface Science, 2007, 12, 166-178.

27. C. Schmitt and S. L. Turgeon, Advances in Colloid and Interface Science, 2011, 167, 63-70.

28. P. Béguin and J.-P. Aubert, FEMS Microbiology Reviews, 1994, 13, 25-58.

29. F. W. Herrick, R. L. Casebier, J. K. Hamilton and K. R. Sandberg, Microfibrillated cellulose: morphology and accessibility, 1983.

30. A. F. Turbak, F. W. Snyder and K. R. Sandberg, Microfibrillated cellulose, a new cellulose product: properties, uses, and commercial potential, 1983.

31. M. P. Lowys, J. Desbrières and M. Rinaudo, Food Hydrocolloids, 2001, 15, 25-32.

32. M. Pääkkö, M. Ankerfors, H. Kosonen, A. Nykänen, S. Ahola, M. Österberg, J. Ruokolainen, J. Laine, P. T. Larsson, O. Ikkala and T. Lindström, Biomacromolecules, 2007, 8, 1934-1941.

33. G. Agoda-Tandjawa, S. Durand, C. Gaillard, C. Garnier and J. L. Doublier, Carbohydrate Polymers, 2012, 87, 1045-1057.

34. A. Karppinen, A.-H. Vesterinen, T. Saarinen, P. Pietikäinen and J. Seppälä, Cellulose, 2011, 18, 1381-1390.

35. D. Tatsumi, H. Kourogi, B. Chen and T. Matsumoto, Colloids and Surfaces A: Physicochemical and Engineering Aspects, 2008, 316, 151-158.

36. Z. Shi, Y. Zhang, G. O. Phillips and G. Yang, Food Hydrocolloids, 2014, 35, 539-545.

37. R. Jonas and L. F. Farah, Polymer Degradation and Stability, 1998, 59, 101-106.

38. A. Kuijk, R. Koppert, P. Versluis, G. van Dalen, C. Remijn, J. Hazekamp, J. Nijsse and K. P. Velikov, Langmuir, 2013, 29, 14356-14360.

39. D. Lin, R. Li, P. Lopez-Sanchez and Z. Li, Food Hydrocolloids, 2015, 44, 435-442.

40. S. J. Veen, A. Kuijk, P. Versluis, H. Husken and K. P. Velikov, Langmuir, 2014, 30, 13362-13368.

41. S. J. Veen, P. Versluis, A. Kuijk and K. P. Velikov, Soft Matter, 2015, 11, 8907-8912.

42. J. De Wit, Journal of Dairy Science, 1998, 81, 597-608.

43. J. N. De Wit, Journal of Dairy Science, 1990, 73, 3602-3612.

44. J. N. deWit and G. Klarenbeek, Journal of Dairy Science, 1984, 67, 2701-2710.

45. D. M. Mulvihill and M. C. F. p. d. Donovan, Irish Journal of Food Science and Technology, 1987, 11, 43-75.

46. A. Brodkorb, T. Croguennec, S. Bouhallab and J. J. Kehoe, in Advanced Dairy Chemistry, Springer, 2016, pp. 155-178.

47. J. I. Boye, I. Alli, A. A. Ismail, B. F. Gibbs and Y. Konishi, International dairy journal, 1995, 5, 337353.

48. A. Clark, G. Kavanagh and S. Ross-Murphy, Food Hydrocolloids, 2001, 15, 383-400.

49. R. Kose, I. Mitani, W. Kasai and T. Kondo, Biomacromolecules, 2011, 12, 716-720. 
50. C. M. Bryant and D. J. McClements, Trends in Food Science \& Technology, 1998, 9, 143-151.

51. M. M. Ould Eleya and S. L. Turgeon, Food Hydrocolloids, 2000, 14, 29-40.

52. E. Saarikoski, T. Saarinen, J. Salmela and J. Seppälä, Cellulose, 2012, 19, 647-659.

53. M. lotti, $\varnothing$. W. Gregersen, S. Moe and M. Lenes, Journal of Polymers and the Environment, 2010, 19, 137-145.

54. A. Okiyama, M. Motoki and S. Yamanaka, Food Hydrocolloids, 1993, 6, 493-501.

55. G. Agoda-Tandjawa, S. Durand, S. Berot, C. Blassel, C. Gaillard, C. Garnier and J. L. Doublier, Carbohydrate Polymers, 2010, 80, 677-686.

56. A. Karppinen, T. Saarinen, J. Salmela, A. Laukkanen, M. Nuopponen and J. Seppälä, Cellulose, 2012, 19, 1807-1819.

57. O. Nechyporchuk, M. N. Belgacem and F. Pignon, Carbohydrate Polymers, 2014, 112, 432-439.

58. T. Saarinen, M. Lille and J. Seppälä, Annu Trans Nord Rheol Soc, 2009, 17, 121-128.

59. Y. Chung and Y. Shyu, International Journal of Food Science \& Technology, 1999, 34, 23-26.

60. H. Ougiya, K. Watanabe, Y. Morinaga and F. Yoshinaga, Bioscience, Biotechnology, and Biochemistry, 1997, 61, 1541-1545.

61. A. Okiyama, M. Motoki and S. Yamanaka, Food Hydrocolloids, 1993, 6, 503-511. 
Chapter 2 


\section{Chapter 3}

Rheology and microstructure of dispersions of protein fibrils and cellulose microfibrils

Submitted as:

Jinfeng Peng, Vincenzo Calabrese, Sandra J. Veen, Peter Versluis, Krassimir P. Velikov, Paul Venema, Erik van der Linden. Rheology and microstructure of dispersions of protein fibrils and cellulose microfibrils. 


\begin{abstract}
Mixtures of whey protein isolate (WPI) and bacterial cellulose (BC) microfibrils after prolonged heating at $80^{\circ} \mathrm{C}$ at $\mathrm{pH} 2$ are investigated. Their macroscopic stability, rheology and microstructure are compared to the unheated (native) mixtures. Results showed that mixtures of WPI and BC microfibrils form stable dispersions when the BC microfibril concentration is $0.2 \mathrm{wt} \%$ and above. The flow curves of the mixtures are comparable to those of the pure $\mathrm{BC}$ microfibril dispersions at the same $\mathrm{BC}$ microfibril concentration. The flow curves show two shear-tinning regions and a plateau region. The mixtures are thixotropic showing hysteresis upon the reversal of the shear flow. After prolonged heating, WPI fibrils are formed in the mixtures and these mixtures show the same stability as the native mixtures. The viscosity of the mixtures is slightly lower compared to the native mixtures, however, the viscosity plateau and the hysteresis are not observed in the mixtures after heating. Mixing BC microfibrils into the WPI solution either before or after heating did not lead to a significant difference in viscosity and microstructure of the mixtures, apart from the observation that the WPI fibrils become shorter when heated in the presence of $B C$ microfibrils.
\end{abstract}




\subsection{Introduction}

The ability to form fibrils is a generic feature of proteins. ${ }^{1,2}$ This has received considerable attention in many research fields ranging from food, ${ }^{3-5}$ biomedical, ${ }^{6-8}$ and material sciences. ${ }^{9-11}$ In foods, fibrils are interesting components due to their multi-functionality including thickening, ${ }^{3}$ gelling, ${ }^{12-14}$ and stabilizing effect. ${ }^{15,16}$ One type of protein that can be used to build fibrils is whey protein. These whey proteins are one of the most studied proteins for food applications, due to their nutritional and functional properties. ${ }^{2}$ When whey proteins are heated at $80^{\circ} \mathrm{C}$ at $\mathrm{pH} 2$ for several hours, the proteins first hydrolyse into peptides and then only part of the peptides will self-assemble into fibrils. ${ }^{17}$ The amount of fibrils and their length distribution are influenced by many factors such as heating time ${ }^{18}$ temperature, ${ }^{19}$ protein concentration, ${ }^{20}$ and even stirring during heating. ${ }^{21}$

Such fibrils built from proteins share a number of structural characteristics with the socalled amyloid fibrils. These amyloid fibrils are associated with a number of the neurodegenerative diseases. ${ }^{6-8,22}$ Therefore, the potential of fibrils, that are built from food proteins, to be used as a functional ingredient in foods has been a major safety issue. ${ }^{23}$ Many studies have been carried out with regards to safety of these fibrils in foods. ${ }^{23-25}$ Bateman et al. ${ }^{26,27}$ studied the digestion of $\beta$-lactoglobulin ( $\beta$-lg) fibrils in vitro and found that the fibrils were digested completely by pepsin within 2 minutes. Shimanovich et al. ${ }^{28}$ found that the protein fibrils are nontoxic to human cells. Recently, Lasse et al. ${ }^{29}$ evaluated the protease resistance and toxicity of amyloid like fibrils from several proteins including whey and did not find any major toxicity of the fibrils towards human cell lines. However, the properties of these fibrils may change, when used as additives in foods due to the complexity of the composition and processing conditions of foods. In addition, it may be that the fibrils are not the damaging species but more the peptides that build the fibrils and/or the peptides that are not built into the fibrils. Therefore, more research still needs to be carried out with regards to safety for use in foods. ${ }^{23}$ In addition, it was stated that these safety studies cannot readily be generalized for fibrils from different sources. ${ }^{23}$

Bacterial cellulose (BC) is a microbial polysaccharide produced from many species of bacteria, among which the Acetobacter xylinus is a widely used model strain in research and industry. ${ }^{30,31}$ It has been demonstrated to be an ideal candidate as a functional 
material in many different fields. ${ }^{30,32-34}$ In foods, it has been shown to be a functional ingredient due to its thickening, gelling, stabilizing, water binding ability etc. ${ }^{35-40}$ In addition, it can be used as a dietary fiber ${ }^{41}$ or a fat-substitute. ${ }^{42,43} \mathrm{BC}$ has a high purity in comparison to plant cellulose and is already used as a food ingredient. For example, BC is the main component of 'Nata de Coco' which is used in low-calorie desserts, salads and foods with high fiber content. It is a gelatinous and chewy material produced in the form of thick sheets of pure cellulose. ${ }^{44}$ The use of $B C$ in foods have been reviewed by Shi et al.. ${ }^{31}$ Despite its multi-functionality, the research of the behaviour of $B C$ in foods has been limited. Studies on the interaction of BC with other ingredients, such as, protein, lipids and oil droplets under different conditions are rather limited.

In this chapter the rheology and microstructure of WPI protein fibrils and BC microfibrils at $\mathrm{pH} 2$ was investigated using a similar approach as used in Chapter 2. The WPI proteins and fibrils are positively charged at $\mathrm{pH} 2$, which is in contrast to $\mathrm{pH} 7$. The concentrations of WPI protein used are also much lower than that of $\mathrm{pH}$ 7. This is because already at very low fibril concentrations a nematic phase arises. ${ }^{45-47}$ The BC microfibrils used are prepared from the commercially available 'Nata de Coco' through high-energy de-agglomeration. A detailed characterization of BC microfibrils obtained from Nata de Coco can be found elsewhere. ${ }^{48}$ On a structural level the $\mathrm{BC}$ consists of bundles of microfibrils, flocs and voids, forming a heterogeneous dispersion. The aim of the study is to investigate how the presence of $B C$ microfibrils influences the microstructure and rheology of the WPI fibrils formed upon prolonged heating and vice versa, i.e. how the protein based fibrillisation and presence of fibrils influences the $\mathrm{BC}$ microstructure and rheology. Recently it was reported that the addition of sodium carboxymethyl cellulose (CMC) to $B C$ dispersions before the de-agglomeration process resulted in a more homogeneous dispersions after de-agglomeration. ${ }^{49,50}$ In our study, WPI is mixed with the BC dispersion after the deagglomeration process. 


\subsection{Materials and methods}

\subsubsection{Whey protein isolate (WPI) solution}

WPI was purchased from Davisco (Bipro, lot \# JE 198 -1-420, USA). The powder was dissolved in an $\mathrm{HCl}$ solution of $\mathrm{pH} 2$ to prepare a WPI stock solution of $15 \mathrm{wt} \%$. The solution was left at $4{ }^{\circ} \mathrm{C}$ with stirring overnight for complete hydration. Afterwards, the $\mathrm{pH}$ of the WPI stock solution was adjusted to $\mathrm{pH} 2$ using a $6 \mathrm{M} \mathrm{HCl}$ solution, followed by a filtration step using a syringe filter (Hydrophilic PES $0.45 \mu \mathrm{m}$, Millipore Millex-HP). Subsequently, the final concentration of the WPI in the solution was characterized using UV spectrophotometer (Cary 50 Bio, Varian) and a calibration curve of known WPI concentration at a wavelength of $278 \mathrm{~nm}$. The stock solution was kept at $4^{\circ} \mathrm{C}$ for further use.

\subsubsection{Bacterial cellulose $(\mathrm{BC})$ microfibril dispersion}

The preparation procedure for the dispersion of $\mathrm{BC}$ microfibrils was the same as described in Chapter 2 on the basis of previous literature. ${ }^{48,49}$ The BC microfibrils were extracted from the BC cubes in the Nata de Coco syrup (Kara Santan Pertama, Bogor 16964, Indonesia) by 8 washing steps. Each step consisted of rinsing the cellulose by filtration over a vacuum filter (Whatman Schleicher and Schuell 113, wet-strengthened circles, 185 $\mathrm{mm}$ in diameter) and redispersing the residue in $1.5 \mathrm{~L}$ of deionized water with the hand blender. For a more homogeneous dispersion, the dispersion was treated by passing once through a high pressure homogeniser (Microfluidizer ${ }^{\mathrm{TM}}$ (MF), Microfluidics - M 110S) with a Z-chamber of $87 \mu \mathrm{m}$ at a pressure of $1.2 \times 10^{5} \mathrm{kPa}$. The concentration of the obtained dispersions was determined by drying approximately $20 \mathrm{~g}$ of dispersion in a vacuum oven. The concentration of the stock BC microfibril dispersion is $1 \mathrm{wt} \%$. The dispersion was stored at $4^{\circ} \mathrm{C}$ before sample preparation.

\subsubsection{WPI-BC microfibril mixtures}

Mixtures containing WPI and BC microfibrils were prepared using the above WPI stock solution and $\mathrm{BC}$ microfibril dispersion at $\mathrm{pH} 2$. $\mathrm{An} \mathrm{HCl}$ solution of $\mathrm{pH} 2$ was used for sample preparation. The transfer of $\mathrm{BC}$ microfibrils is performed by using a pipette with a cut-off tip. After mixing, the $\mathrm{pH}$ of all mixtures was adjusted to $\mathrm{pH} 2$ using a $6 \mathrm{M} \mathrm{HCl}$ solution. For 
ease of discussion, if not further specified, the concentrations of WPI and BC microfibrils in the mixtures are defined as [WPI, BC] with both in weight percentages (wt\%). On the basis of different pre-treatments, three types of WPI-BC microfibril mixtures were prepared:

\subsubsection{WPI-BC microfibril mixtures before heating (native mixtures)}

The mixtures were prepared by direct mixing of WPI solution and BC microfibril dispersions at $\mathrm{pH}$ 2. The final concentrations of WPI and BC microfibrils in these mixtures are: $[\mathrm{WPI}, \mathrm{BC}]=[0,0.1],[0,0.2],[0,0.3],[2,0.1],[3,0.1],[4,0.1],[4,0.2],[4,0.3],[4,0]$.

\subsubsection{WPI-BC mixtures after heating (heated mixtures)}

The WPI solution and BC microfibril dispersion were mixed directly at different concentration ratios and stirred at $\mathrm{pH} 2$. The mixtures were then heated in a heating plate at $80^{\circ} \mathrm{C}$ for 22 hours at $\mathrm{pH} 2$ with mild stirring. After heating, the mixtures were cooled in ice water and stored at $4{ }^{\circ} \mathrm{C}$ for further measurements. The final concentrations (wt\%) of $\mathrm{WPI}$ and $\mathrm{BC}$ microfibrils in these mixtures are: $[\mathrm{WPI}, \mathrm{BC}]=[0,0.1],[2,0.1],[3,0.1],[4,0.1]$, $[4,0.2],[4,0.3],[2,0],[3,0],[4,0]$.

\subsubsection{The effect of BC microfibrils on WPI fibril formation}

Upon heating at $\mathrm{pH} 2$ at $80{ }^{\circ} \mathrm{C}$ for 22 hours, the WPI fibrils are formed in WPI solution. ${ }^{51}$ The formation process consists of first protein hydrolysis and then the assembly of hydrolyzed peptides into fibrils. ${ }^{17}$ In order to investigate whether the fibril formation process affect the rheology and microstructure of $B C$ microfibrils and vice versa, whether the presence of $B C$ microfibrils affect the rheology and microstructure of the formed WPI fibrils during heating, $B C$ microfibrils were mixed into WPI solution both before and after WPI fibril formation.

\section{(1) Mixing BC microfibrils before heating (BH) the WPI solution}

The WPI and BC microfibrils were directly mixed together and stirred prior to the heating treatment. Afterwards, the mixtures are heated at $80^{\circ} \mathrm{C}$ for 22 hours at $\mathrm{pH} 2$ with mild stirring. The WPI and BC microfibril concentrations in the mixtures are: $[\mathrm{WPI}, \mathrm{BC}]=[2,0.2]$, $[2,0.6],[4,0.2],[4,0.6]$. After heating, the mixtures were cooled down in ice water. Subsequently, the mixtures were diluted two-fold using an $\mathrm{HCl}$ solution of $\mathrm{pH} 2$ and stored 
at $4{ }^{\circ} \mathrm{C}$ for further analysis. The corresponding final concentrations of WPI and $\mathrm{BC}$ microfibrils after dilution are: $[\mathrm{WPI}, \mathrm{BC}]=[1,0.1],[1,0.3],[2,0.1],[2,0.3]$.

\section{(2) Mixing $B C$ microfibrils after heating (AH) the WPI solution}

Prior to mixing, the WPI solution and BC microfibril dispersion were heated separately at $80^{\circ} \mathrm{C}$ for 22 hours at $\mathrm{pH} 2$ with mild stirring. The concentrations of WPI solutions heated were 2 and 4 wt\%. The concentrations of BC microfibril dispersions heated were 0.2 and 0.6 wt\%. After being heated, the samples were cooled down in ice water. Subsequently, the WPI and BC microfibrils were mixed together and stirred. The corresponding final concentrations $(w t \%)$ after mixing are: $[\mathrm{WPI}, \mathrm{BC}]=[1,0.1],[1,0.3],[2,0.1],[2,0.3]$.

Prior to measurements, all mixtures were stirred for $30 \mathrm{~min}$ at room temperature. Samples are all in liquid state both before and after heating.

\subsubsection{Visual observation}

Images were taken at time 0,1 and 7 days after sample preparation to macroscopically monitor the stability of the samples over time at $4{ }^{\circ} \mathrm{C}$.

\subsubsection{Viscosity}

A stress-controlled rheometer (MCR 302, Anton Paar) with sandblasted concentric cylinder geometry (CC17/TI/S-SN38492) was used for measuring the viscosity of the samples. The use of a sandblasted geometry is to minimize the effect of wall slip. The transfer of samples to the rheometer was done using a pipette with a cut-off tip to minimise BC microfibril alignment. The viscosity was measured by first applying a preshear at a frequency of $1 \mathrm{~Hz}$ and strain of $0.1 \%$ for $5 \mathrm{~min}$. Subsequently, the viscosity was measured by increasing the shear rate from 0.1 to $5001 / \mathrm{s}$ in $2 \mathrm{~min}$ and then decreasing from 500 to $0.11 / \mathrm{s}$ in $2 \mathrm{~min}$.

\subsubsection{Confocal laser scanning microscopy (CLSM)}

Calcofluor White and Rhodamine B were used to stain the BC microfibrils and WPI, respectively. In $1 \mathrm{ml}$ sample, $5 \mu \mathrm{l}$ of $0.05 \%$ Calcofluor white and $5 \mu \mathrm{l}$ of 0,002\% Rhodamine $\mathrm{B}$ were added prior to imaging. About $20 \mu \mathrm{l}$ sample was transferred to a chamber built 
from a microscopic glass slide and a cover slip. The images were taken on a Zeiss LSM 510 Meta microscope (Zeiss, Germany) equipped with an Axiovert 200M inverted microscope (Wetzlar, Germany). An oil immersion objective (EC Plan-Neoufluar $40 \times / 1.30$ Oil DIC) was used for imaging. The excitation and emission wavelength used for Rhodamine B are 543 $\mathrm{nm}$ and $560 \mathrm{~nm}$. The excitation and emission wavelength used for Calcofluor white are $405 \mathrm{~nm}$ and $420 \mathrm{~nm}$.

For WPI-BC microfibril mixtures prepared by mixing BC microfibrils before and after heating the WPI solution, the samples are stained by Congo red. Congo red is able to stain both WPI fibrils and BC microfibrils. To do this, a small drop of Congo red (1 wt\%) was added to $1 \mathrm{ml}$ of the sample. The excitation wavelength and emission wavelength are 514 $\mathrm{nm}$ and $670 \mathrm{~nm}$. Images were taking on a microscope ( LSM 5 EXCITER, Carl Zeiss) equipped with an Axiovert $200 \mathrm{M}$ inverted microscope (Thornwood, NY) using the oil immersion objective (Plan-Apochromat, 100x/1.46 oil, Zeiss, Germany).

\subsubsection{Transmission electron microscopy (TEM)}

The samples of WPI-BC microfibril mixtures were diluted 20 -fold using an $\mathrm{HCl}$ solution of $\mathrm{pH} 2$ before imaging. Glow discharge was applied on the carbon copper TEM grid to make the surface hydrophilic. A drop of sample was put a TEM grid and then was dried by a filter paper after 15s. A drop of $2 \%$ phosphortungsten acid was added on the grid to stain the sample. The excess water was removed by another filter paper, followed by air drying before imaging. TEM images were taken on a transmission electron microscope (Tecnai 20 system from $\mathrm{FEI})$.

TEM images of the only WPI fibrils after centrifugation from WPI-BC microfibril mixtures were also imaged using a transmission electron microscope (JEOL JEM-1011, Tokyo, Japan). To do this, the mixtures were first centrifuged at 3350 RCF for $30 \mathrm{~min}$ to spin down the BC microfibrils. The WPI fibrils in the supernatant were then taken for imaging. The TEM preparation is similar to the procedure described above, only in which the WPI fibrils in the supernatant was stained by adding a drop of $2 \%$ uranyl acetate onto the grid. 


\subsubsection{Scanning electron microscopy (SEM)}

A droplet of the sample was put on nuclepore polycarbonate membrane ( $1 \mathrm{~mm}$ holes, Costar) A filter paper was placed under the membrane to absorb the liquid through the membrane. The samples were then air dried. Subsequently, the membrane were glued on sample holders by carbon adhesive tabs (EMS, Washington, USA) and sputter coated with $10 \mathrm{~nm}$ Iridium (Leica EM SCD 500, Vienna, Austria). A high resolution scanning electron microscope (Magellan 400, FEI, Eindhoven, the Netherlands) was used for imaging.

To perform Cryo-SEM, a droplet of the sample was placed on a hollow copper rivet and rapidly frozen in liquid ethane. The rivets were put in a cryo-sample holder in liquid nitrogen and subsequently transferred to the cryo-preparation system (MED 020/ VCT 100, Leica, Vienna, Austria) onto the sample stage. The samples were then fractured, freeze dried for $10 \mathrm{~min}$ and sputter coated with a layer of $10 \mathrm{~nm}$ tungsten at the same conditions. Imaging was performed after cryo-shielding and transferring the sample onto the sample stage of the field emission scanning microscope (Magellan 400, FEl, Eindhoven, The Netherlands).

\subsection{Results and discussion}

\subsubsection{Visual observation}

The macroscopic stability of the pure BC microfibril dispersions, pure WPI solutions and WPI-BC microfibril mixtures was monitored by taking images over a time period of 7 days. In the case of native samples (cf. Figure 1), the pure 0.1 wt\% BC microfibril dispersion showed some limited sedimentation after 7 days. When the concentration of $B C$ microfibrils was at and above $0.2 \mathrm{wt} \%$, the $\mathrm{BC}$ microfibril dispersions remained stable against sedimentation for 7 days. Mixing WPI up to 3 wt\% with BC microfibril dispersion showed a similar sedimentation as the pure 0.1 wt\% BC microfibril dispersion. The sedimentation rate became slower when 4 wt\% WPI is added to the $0.1 \mathrm{wt} \% \mathrm{BC}$ microfibril dispersion. In WPI-BC microfibril mixtures containing BC microfibril concentration at and above 0.2 wt\%, the mixtures remained stable, i.e. similar to those of the pure $B C$ microfibril dispersions at the same concentration. The sedimentation of $B C$ microfibril dispersions at low concentrations is in line with an earlier study, ${ }^{48}$ where it was found that $\mathrm{BC}$ microfibrils forms a collapsed gel at low concentrations. Above a certain BC microfibril 
concentration, no sedimentation could be observed. It can be concluded that the mixtures of WPI and BC microfibrils form a stable dispersion when BC concentration is larger than $0.2 \mathrm{wt} \%$ at $\mathrm{pH} 2$.

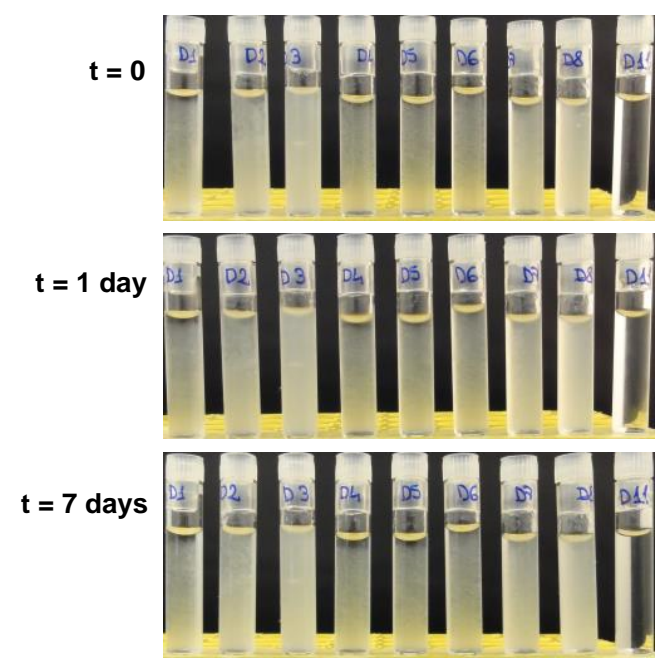

Figure 1 Images of native WPI-BC microfibril mixtures at time 0,1 day and 7 days after preparation at $4^{\circ} \mathrm{C}$. The concentrations (in wt\%) are: $[\mathrm{WPI}, \mathrm{BC}]=[0,0.1],[0,0.2],[0,0.3],[2,0.1],[3,0.1],[4$, $0.1],[4,0.2],[4,0.3],[4,0]$ corresponding to the tubes from left to right.

After being heated at $80^{\circ} \mathrm{C}$ for 22 hours at $\mathrm{pH} 2$ (Figure 2), the stability of the pure $\mathrm{BC}$ microfibril dispersions, pure WPI solutions and the mixtures of WPI-BC microfibrils was observed to be similar to the native samples in Figure 1. In samples containing 0.1 wt\% BC microfibrils, sedimentation could be observed, while at and above $0.2 \mathrm{wt} \%$ BC microfibrils, the samples remained stable for 7 days. From this, it can be concluded that the prolonged heating treatment did not influence the macroscopic stability of the samples. 


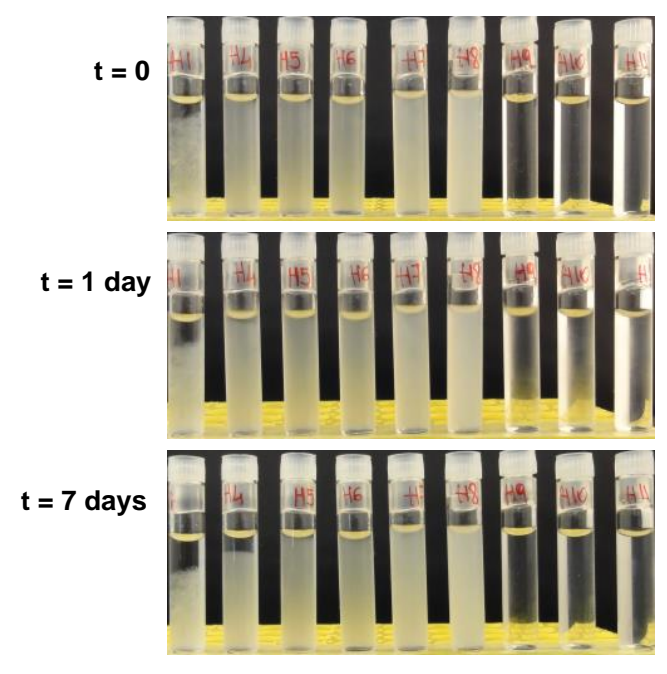

Figure 2 Images of WPI-BC microfibril mixtures after heating at time 0,1 day and 7 days after preparation at $4{ }^{\circ} \mathrm{C}$. The concentrations (in wt\%) are: $[\mathrm{WPI}, \mathrm{BC}]=[0,0.1],[2,0.1],[3,0.1],[4,0.1]$, $[4,0.2],[4,0.3],[2,0],[3,0],[4,0]$ corresponding to the tubes from left to right.

Upon heating at $80{ }^{\circ} \mathrm{C}$ at $\mathrm{pH} 2$ for 22 hours, protein fibrils are formed in the pure WPI solutions. ${ }^{18}$ The formation process is initiated by protein hydrolysis into peptides, followed by the assembly of peptides into fibrils. ${ }^{17}$ To investigate whether the presence of $\mathrm{BC}$ microfibrils affect the rheology and microstructure of the WPI fibrils, and vice versa, the $B C$ microfibrils are mixed both before heating $(\mathrm{BH})$ and after heating $(\mathrm{AH})$ the WPI solution. In Figure 3, it is observed that the stability of the WPI-BC microfibril mixtures did not change significantly when BC microfibrils are mixed with WPI either before or after heating the WPI solution. In mixtures containing 0.1 wt\% BC microfibril, sedimentation of $\mathrm{BC}$ microfibrils was observed, while in mixtures containing $0.3 \mathrm{wt} \% \mathrm{BC}$, no sedimentation could be observed. The concentration of WPI did not show influence on the macroscopic stability of the mixtures. It can be concluded that the WPI and BC form stable mixtures at 0.3 wt\% BC either mixing BC microfibrils before or after heating the WPI solution. 


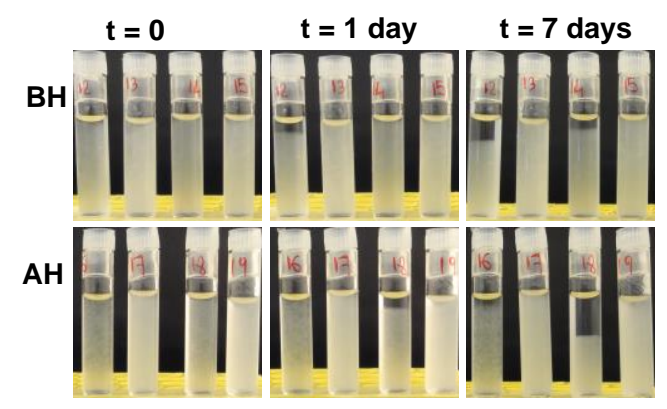

Figure 3 Images of WPI-BC microfibril mixtures mixing before heating (BH) and after heating (AH) the WPI solution at time 0,1 day and 7 days after preparation at $4{ }^{\circ} \mathrm{C}$. The concentrations (in wt\%) are: $[\mathrm{WPI}, \mathrm{BC}]=[1,0.1],[1,0.3],[2,0.1],[2,0.3](\mathrm{BH})$ and $[1,0.1],[1,0.3],[2,0.1],[2,0.3](\mathrm{AH})$ corresponding to the tubes from left to right.

\subsubsection{Rheological properties}

\subsubsection{Native WPI-BC microfibril mixtures}

The elastic modulus $G^{\prime}$ and loss modulus $G^{\prime \prime}$ were measured as a function of time for the native samples (Figure 4). The $\mathrm{G}^{\prime}$ and $\mathrm{G}^{\prime \prime}$ of the pure BC microfibril dispersions are close to those of the WPI-BC microfibril mixtures at the same BC microfibril concentrations. The $\mathrm{G}^{\prime}$ is increased and $G^{\prime \prime}$ is decreased initially, which is a typical characteristic of BC microfibril dispersions, caused by reformation of the microfibrils bundles after the loading of the geometry. ${ }^{50}$ The $\mathrm{G}^{\prime}$ and $\mathrm{G}^{\prime \prime}$ of the native mixtures are mainly determined by the presence of BC microfibrils.

The viscosity of the pure BC microfibril dispersions (Figure 5a) showed a strong shear thinning and thixotropic behaviour, in line with previous studies ${ }^{48,50,52}$ and with the results in Chapter 2. The viscosity increases with increasing BC microfibril concentration which was attributed to the formation of a denser network formed by the entanglements of the microfibrils. ${ }^{48}$ Interestingly, a viscosity plateau (between shear rates of 1 and 10 1/s) is observed between two shear thinning regions and was also reported previously. ${ }^{53}$ This variation is exemplified in the behaviour of the shear stress (Figure $5 b$ ) and is in agreement with the results from Kuijk et al. ${ }^{48}$ This phenomenon is similar to the flow behaviour of microfibrillated cellulose dispersions originating from plants and wood, which was shown to be caused by flocculation of the cellulose microfibrils under shear. ${ }^{54-59}$ 
Similar flow curves were found in mixtures of $B C$ microfibrils and carboxymethyl cellulose $(\mathrm{CMC})$, in which the underlying mechanism was proposed to be the structural heterogeneities of the cellulose microfibrils. ${ }^{52}$

Upon the reversal of shear rate, the viscosity of the pure BC microfibril dispersions is lower compared to the viscosity when the shear rate was increased. This hysteresis was also found at $\mathrm{pH} 7$ in Chapter 2 and also reported for micro-fibrillated wood cellulose. ${ }^{54}$

Upon mixing WPI into BC microfibril dispersions, the viscosity and shear stress behaved almost identical to those of the pure $\mathrm{BC}$ microfibril dispersions at the same $\mathrm{BC}$ microfibril concentrations. In addition, the hysteresis effect also existed in the mixtures. The viscosity of pure WPI is low in comparison to pure BC microfibril dispersions and pure WPI solution does not exhibit hysteresis upon reversed shear rate. Therefore, it can be concluded that the viscosity of the mixtures is dominated by the $\mathrm{BC}$ microfibrils.
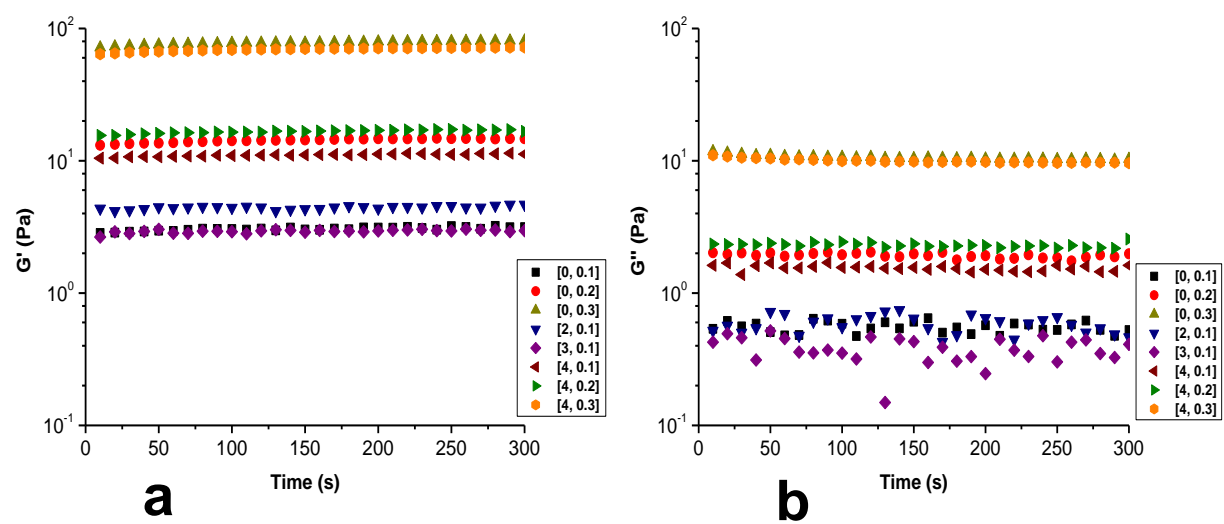

Figure 4 Storage modulus G' (a) and loss modulus G" (b) of native samples as a function of time. The concentrations (in wt\%) are: $[\mathrm{WPI}, \mathrm{BC}]=[0,0.1],[0,0.2],[0,0.3],[2,0.1],[3,0.1],[4,0.1],[4$, $0.2]$, [4, 0.3]. 

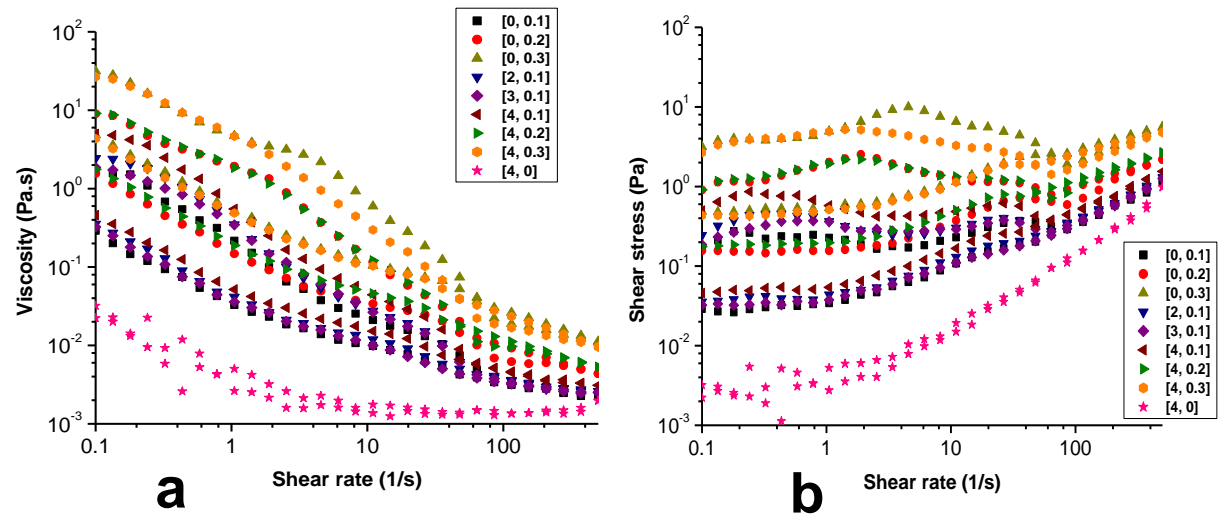

Figure 5 Viscosity (a) and shear stress (b) of native samples as a function of shear rate. The shear rate is first increased from 0.1 to $5001 / \mathrm{s}$, and then decreased from 500 to $0.1 \mathrm{1} / \mathrm{s}$. The concentrations (in wt\%) are: $[\mathrm{WPI}, \mathrm{BC}]=[0,0.1],[0,0.2],[0,0.3],[2,0.1],[3,0.1],[4,0.1],[4,0.2]$, $[4,0.3],[4,0]$.

\subsubsection{WPI-BC mixtures after heating}

After heating at $80^{\circ} \mathrm{C}$ for 22 hours, the elastic modulus $\mathrm{G}^{\prime}$ and loss modules $\mathrm{G}^{\prime \prime}$ of the pure 0.1 wt\% BC microfibril dispersion was slightly lower compared to the native BC microfibril dispersions (Figure 4). This indicates that the $\mathrm{G}^{\prime}$ and $\mathrm{G}^{\prime \prime}$ of the $\mathrm{BC}$ microfibril dispersions was not substantially modified by prolonged heating. The pure WPI solutions form protein fibrils after heating ${ }^{18}$ and $G^{\prime}$ and $G^{\prime \prime}$ of the 2 to 4 wt\% samples are close to each other. In the WPI-BC microfibril mixtures, the $G^{\prime}$ and $G^{\prime \prime}$ were also slightly lower than that of the native samples, implying that the heating did not significantly influence the $G^{\prime}$ and $G^{\prime \prime}$ values of the samples. The change of $G^{\prime}$ and $G^{\prime \prime}$ over time was similar to the native samples, indicating that the presence of $B C$ microfibrils dominants the rheological properties of the mixtures. 

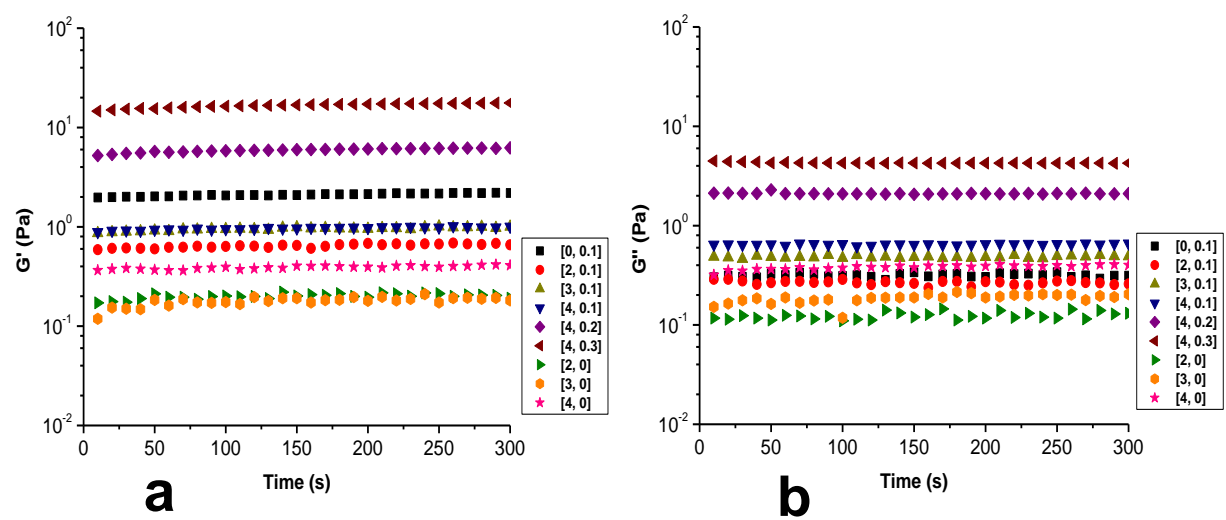

Figure 6 Storage modulus G' (a) and loss modulus G' (b) as a function of time of WPI-BC microfibril mixtures after heating together at $80^{\circ} \mathrm{C}$ for 22 hours. The concentrations (in wt\%) are: [WPI, BC] = $[0,0.1],[2,0.1],[3,0.1],[4,0.1],[4,0.2],[4,0.3],[2,0],[3,0],[4,0]$.

The viscosity of the pure BC microfibril (0.1 wt\%) dispersion after heating showed the same behavior as the native $B C$ microfibril dispersion, i.e. strong shear thinning behavior is observed and also the viscosity plateau exists between the two shear thinning regions. The hysteresis, i.e. lower viscosity upon shear flow reversal is also present. This implies that the prolonged heating at $\mathrm{pH} 2$ did not significantly modify the rheological behavior of the BC microfibril dispersions. The pure WPI solutions after heating showed also strong shear thinning behavior and viscosity is increased with increasing WPI concentration, which is in line with the literature. ${ }^{3}$ The viscosity of the WPI-BC microfibril mixtures after heating showed strong shear thinning, however, the viscosity plateau was not observed in these mixtures (cf. Figure 7). Upon a shear flow reversal, the viscosity was the same as the initial value when the shear rate was increased, i.e. no hysteresis was observed. The viscosity of the mixtures after heating is slightly lower than that of the native mixtures and is mainly controlled by the presence of the BC microfibrils. However, the presence of WPI after heating (i.e. fibril formation) changed the flow behavior of the BC microfibril dispersions, as the viscosity plateau and hysteresis are specific features of pure $\mathrm{BC}$ microfibril dispersions. 

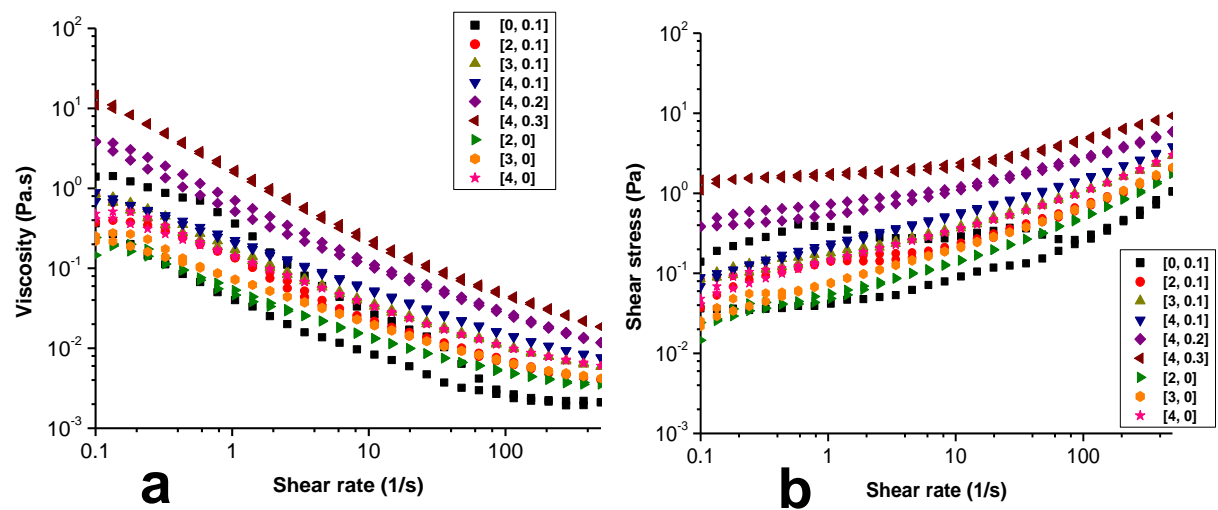

Figure 7 Viscosity (a) and shear stress (b) as a function of shear rate of WPI-BC mixtures after heating at $80^{\circ} \mathrm{C}$ for 22 hours. The shear rate is first increased from 0.1 to $5001 /$, and then decreased from 500 to 0.1 1/s. The concentrations (in wt\%) are: $[\mathrm{WPI}, \mathrm{BC}]=[0,0.1],[2,0.1],[3$, $0.1],[4,0.1],[4,0.2],[4,0.3],[2,0],[3,0],[4,0]$.

\subsubsection{Mixing $B C$ microfibrils before $(B H)$ and after heating $(A H)$ WPI solution}

Comparing the mixtures prepared by mixing $B C$ microfibrils before and after heating the WPI solution, the elastic modulus $G^{\prime}$ and loss modulus $G^{\prime \prime}$ (Figure 8) did not show much difference. The mixtures containing the same $\mathrm{BC}$ microfibril concentrations showed almost the same $G^{\prime}$ and $G^{\prime \prime}$, regardless of the WPI concentration. The rheological properties are dominated by the $\mathrm{BC}$ microfibrils. Mixing $\mathrm{BC}$ microfibril dispersion before or after heating the WPI solutions gave identical results.

The viscosity and shear stress of the mixtures prepared by mixing BC microfibrils both before and after heating the WPI solution are compared (cf. Figure 9). For mixtures containing the same concentration of $\mathrm{BC}$ microfibrils, the values of viscosity and shear stress as a function of shear rate were comparable to each other, mixing BC microfibrils either before or after heating the WPI solution. However, we did observe the viscosity plateau and hysteresis present in those mixtures, which is in contrast to our heated mixtures as shown in Figure 7. Although they are all heated, it is noted that samples in Figure 9 were diluted after heating for rheological measurements. The samples that were mixed before heating were diluted two-fold after heating to have the same final 
concentrations as the samples that were mixed after heating. It is concluded that the mixing $B C$ dispersions before or after heating the WPI solution did not show a significant difference.
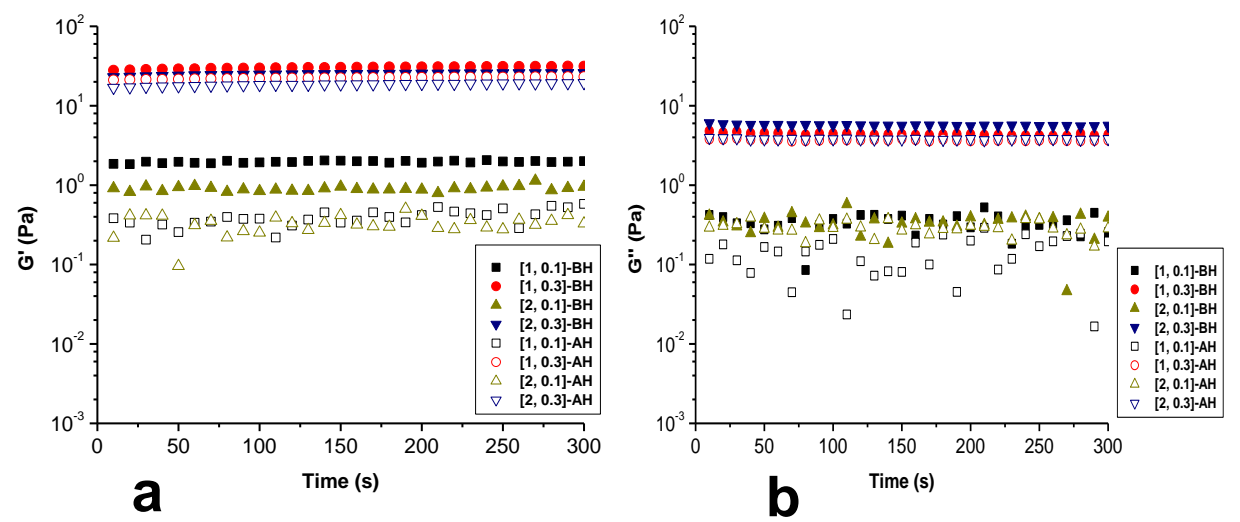

Figure 8 Storage modulus G' (a) and loss modulus G" (b) as a function of time of WPI-BC microfibril dispersions prepared by mixing $\mathrm{BC}$ microfibrils before heating ( $\mathrm{BH}$, filled symbols) and after heating (AH, open symbols) WPI solution. The concentrations (in wt\%) are: $[\mathrm{WPI}, \mathrm{BC}]=[1,0.1],[1$, $0.3],[2,0.1],[2,0.3]$. 

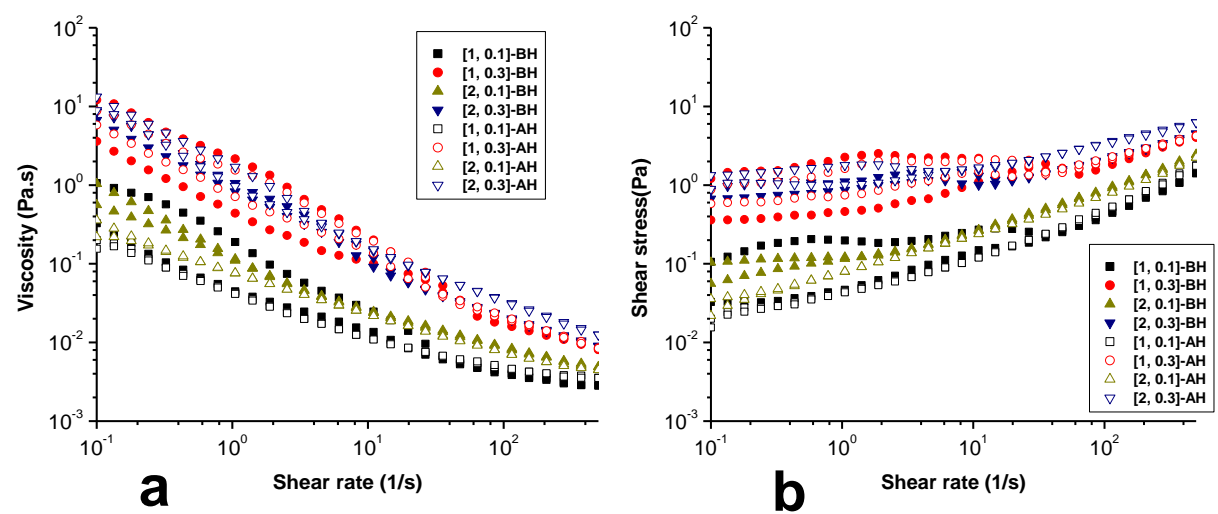

Figure 9 Viscosity (a) and shear stress (b) as a function of shear rate of WPI-BC microfibril dispersions prepared by mixing $\mathrm{BC}$ microfibrils before heating ( $\mathrm{BH}$, filled symbols) and after heating (AH, open symbols) the WPI solution. The shear rate is first increased from 0.1 to 5001 /, and then decreased from 500 to $0.11 / \mathrm{s}$. The concentrations (in wt\%) are: [WPI, BC] = [1, 0.1], [1, $0.3],[2,0.1],[2,0.3]$.

\subsubsection{Microstructure}

The microstructure of the samples was characterized using TEM, CLSM and SEM to investigate whether the presence of $B C$ microfibrils influence the WPI fibril structure formed during heating and vice versa, whether the WPI fibril formation process affects the microstructure of the $\mathrm{BC}$ microfibrils.

\subsubsection{Transmission electron microscopy (TEM)}

Figure 10 shows the TEM images of the mixtures without centrifugation and Figure 11 shows the TEM images of only WPI fibrils after centrifugation.

In the pure WPI solution, long and thin protein fibrils are formed during heating (cf. Figure 10). In the mixtures where BC microfibrils are mixed with the WPI solution before heating, shorter fibrils are formed in comparison to the pure WPI solution. The concentration of WPI in both samples is (4 wt\%). In the mixtures where BC microfibrils are mixed after heating the WPI solution, one can observe long fibrils in those samples, regardless of the $\mathrm{BC}$ microfibril concentrations. The WPI fibrils formed in those mixtures are comparable to 
the pure WPI solution, as both of the samples was heated alone at the same WPI concentration for fibril formation. It can be concluded that the presence of BC microfibrils influenced the fibril formation, i.e. on the average shorter fibrils are formed, although the length distribution was not quantified. This observation was confirmed by the TEM images of the WPI fibrils obtained after centrifugation of the mixtures (cf. Figure 11). For the four concentration ratios (Figure 11), it is observed that mixing $B C$ microfibrils before heating $(\mathrm{BH})$ the WPI solution generated shorter fibrils than that mixing after heating $(\mathrm{AH})$ the WPI solution. In the $\mathrm{BH}$ samples for both $\mathrm{BC}$ microfibril concentrations of $0.1 \%$ and $0.3 \%$, it was observed that mixtures with $1 \mathrm{wt} \%$ WPI (initial $2 \mathrm{wt} \%$ for fibril formation) resulted in shorter fibrils than for the samples with 2 wt\% WPI (initial 4 wt\% for fibril formation). However, in AH samples, where BC microfibrils are added after WPI fibril formation, this is not observed. No clear difference of the fibril length between mixtures containing 1 wt\% and 2 wt\% WPI can be concluded in the AH samples. In addition, in both BH and $\mathrm{AH}$ samples, It was observed that the fibrils become shorter when the BC microfibril concentration increases (cf. Figure 11). This may be because the fibrillisation process is influenced by the presence of $\mathrm{BC}$ and/or that the longer fibrils are being sedimented together with the $\mathrm{BC}$ during centrifugation. 


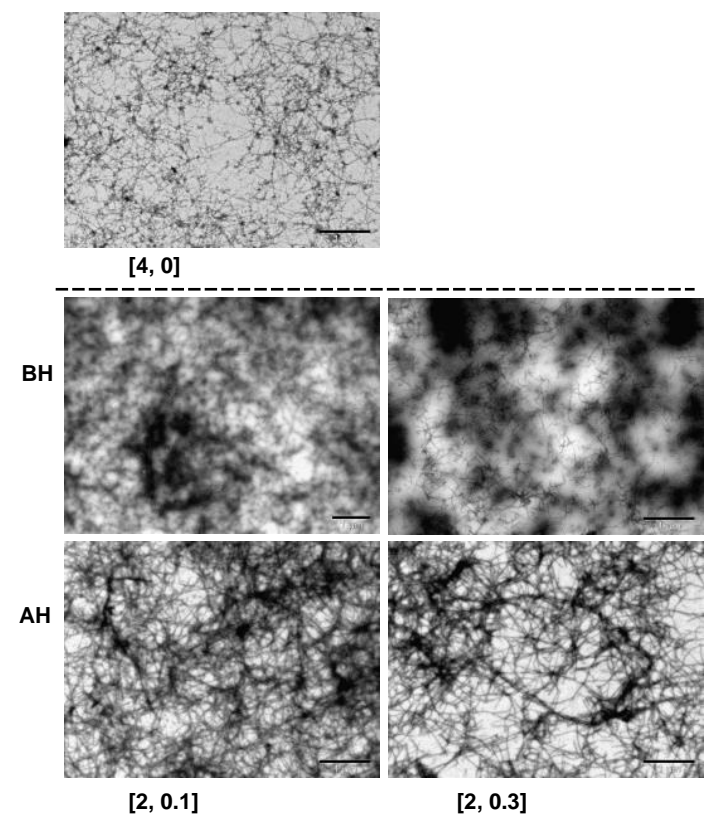

Figure 10 TEM images of mixtures prepared by mixing $B C$ microfibrils before heating (BH) and after heating (AH) WPI solution (without centrifugation). Top line includes images of WPI being heated alone. The concentrations (in wt\%) are: $[\mathrm{WPI}, \mathrm{BC}]=[4,0]$ and $[2,0.1],[2,0.3]$ for mixtures. The images show the WPI fibrils formed upon heating. Scale bar corresponds to $1 \mu \mathrm{m}$.

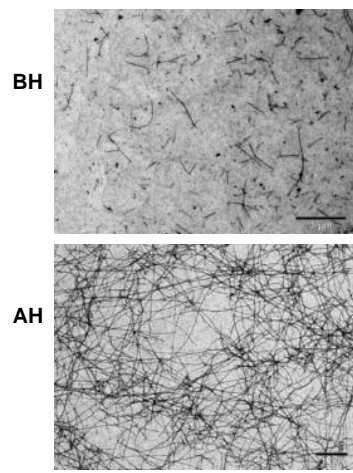

$[1,0.1]$
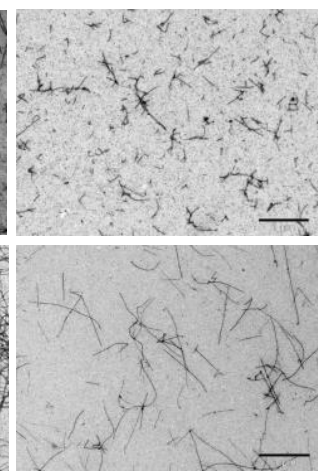

$[1,0.3]$

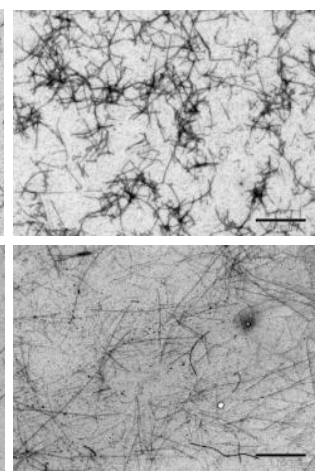

$[2,0.1]$

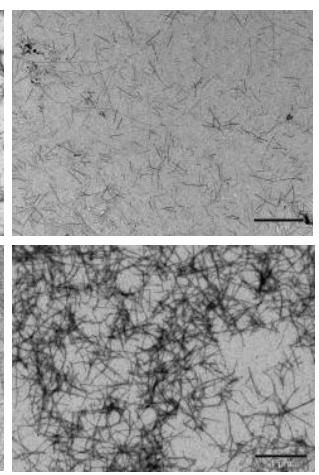

$[2,0.3]$

Figure 11 TEM images of only WPI fibrils after centrifugation of mixtures prepared by mixing BC microfibrils before heating (BH) and after heating (AH) WPI solution. Scale bar corresponds to 1 $\mu \mathrm{m}$. The concentrations (in wt\%) are: [WPI, BC] = [1, 0.1], [1, 0.3], [2, 0.1], [2, 0.3]. 


\subsubsection{Confocal laser scanning microscopy (CLSM)}

The flocs of BC microfibrils are clearly visible in the CLSM images in Figure 12 and show a variation in size. In the WPI-BC microfibril mixtures, the WPI particles are too small to be visible. The structures of the BC microfibrils as observed by CSLM did not show significant differences as a function of the WPI concentration. In the samples after heating in Figure 13 , the WPI fibrils are homogeneously distributed. The BC microfibril structures are similar as observed in the native samples. In the mixtures prepared by mixing BC microfibrils before and after heating the WPI solution, Congo red binds to both WPI fibrils and BC microfibrils. WPI fibrils are homogeneously distributed, whereas the BC microfibrils are shown in flocs. No significant difference on the microstructure of the $\mathrm{BH}$ and $\mathrm{AH}$ samples can be observed.

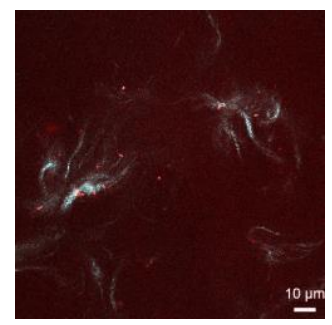

$[0,0.1]$

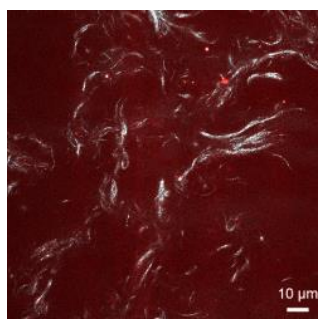

$[0,0.3]$

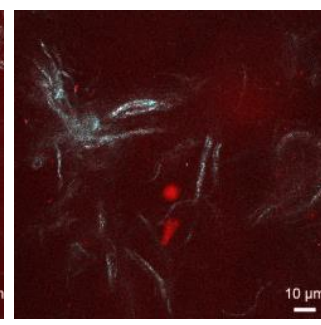

$[2,0.1]$

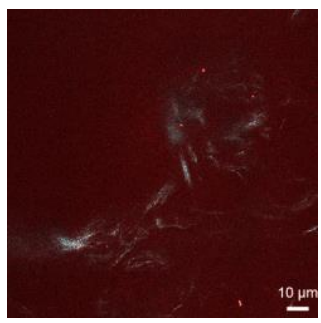

$[4,0.1]$

Figure 12 CLSM images of native pure BC microfibril dispersions and WPI-BC microfibril mixtures. WPI is indicated as red and $B C$ microfibrils are indicated as turquoise. Scale bar $=10 \mu \mathrm{m}$. The concentrations (in wt\%) are: $[\mathrm{WPI}, \mathrm{BC}]=[0,0.1],[0,0.3],[2,0.1],[4,0.1]$. 


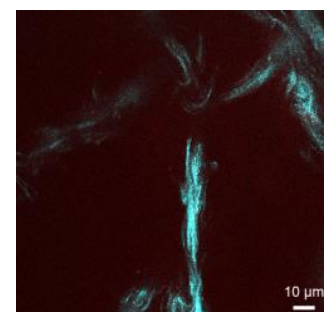

$[0,0.1]$

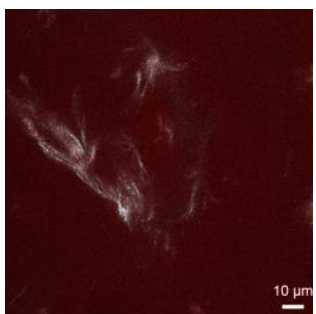

$[2,0.1]$

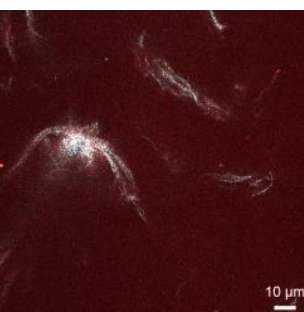

$[4,0.1]$

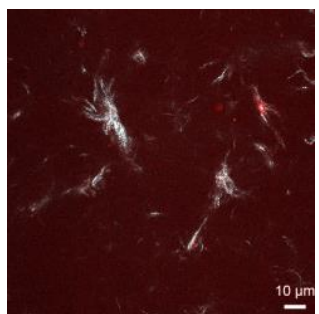

$[4,0.3]$

Figure 13 CLSM images of heated pure BC microfibrils dispersion and WPI-BC microfibrils mixtures. WPI is indicated as red and BC microfibrils are indicated as turquoise. Scale bar $=\mathbf{1 0} \mu \mathrm{m}$. The concentrations (in wt\%) are: $[\mathrm{WPI}, \mathrm{BC}]=[0,0.1],[2,0.1],[4,0.1],[4,0.3]$. 


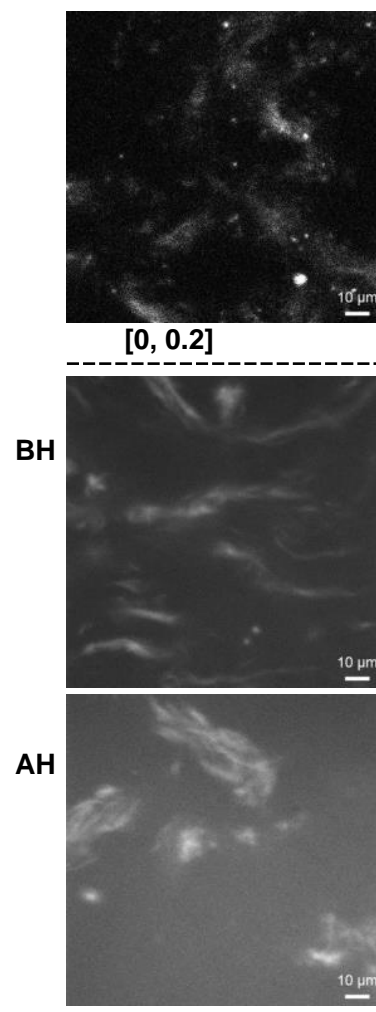

$[2,0.1]$

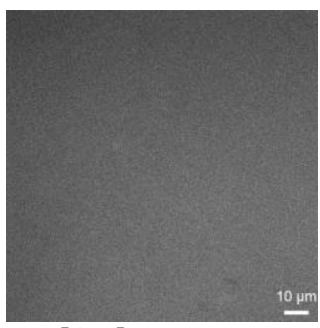

$[4,0]$
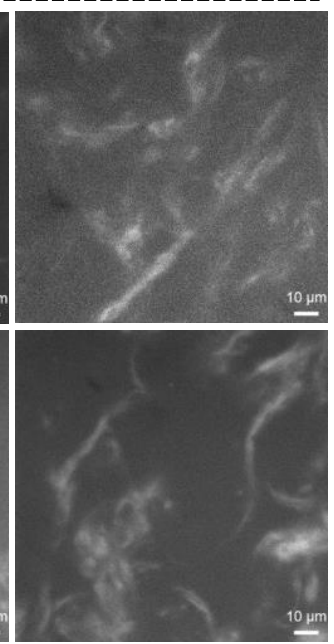

$[2,0.3]$

Figure 14 CLSM images of mixtures prepared by mixing $B C$ microfibrils before heating (BH) and after heating (AH) the WPI solution. Top line includes images of WPI and BC microfibrils being heated alone. The concentrations (in wt\%) are: $[\mathrm{WPI}, \mathrm{BC}]=[0,0.2],[4,0]$ and $[2,0.1],[2,0.3]$ for mixtures. The samples are stained by Congo red.

\subsubsection{Scanning electron microscopy (SEM)}

The pure $B C$ microfibril dispersion after prolonged heating shows $B C$ microfibrils present in the form of bundles (cf. Figure 15). A similar image was found for unheated BC in the study of Kuijk et al. ${ }^{48}$ It can be concluded that no significant microstructure change of the $\mathrm{BC}$ microfibrils occurred upon prolonged heating at $\mathrm{pH}$ 2. The SEM image of the WPI (only) after heating is shown in Figure 16, in which it shows the characteristic long and linear fibril structure. Comparing the WPI-BC microfibril mixtures mixing before and after heating, i.e. $\mathrm{BH}$ versus $\mathrm{AH}$, samples prepared by air drying on filter paper (cf. Figure 16b) 
do not reveal differences. However, the cryo-SEM images (cf. Figure 16 a) do show a difference where it seems that the $\mathrm{AH}$ sample looks more like that of an ordinary protein based fibrillar gel (cf. Figure 16a of protein fibrils alone), while the $\mathrm{BH}$ seems to contain small strands "sticking out" of the main fibrillar structures. The latter presumably consist of $\mathrm{BC}$ microfibrils together with protein.

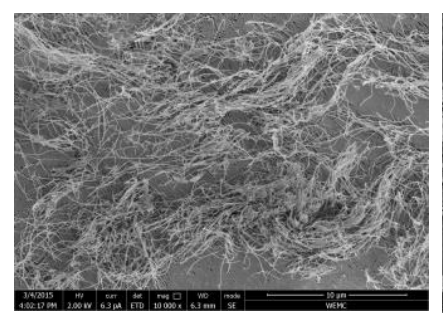

a

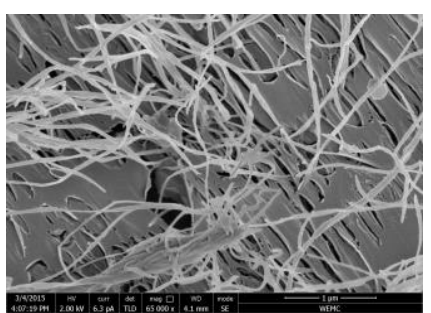

b

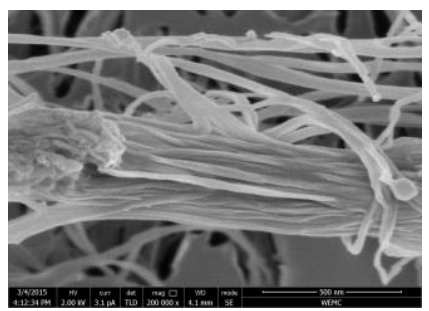

C

Figure 15 SEM images of $0.2 \mathrm{wt} \% \mathrm{BC}$ microfibrils after being heated for 22 hours at $80^{\circ} \mathrm{C}$ at $\mathrm{pH} 2$. Scale bar corresponds to $10 \mu \mathrm{m}$ (a), $1 \mu \mathrm{m}$ (b) and $500 \mathrm{~nm}$ (c). 


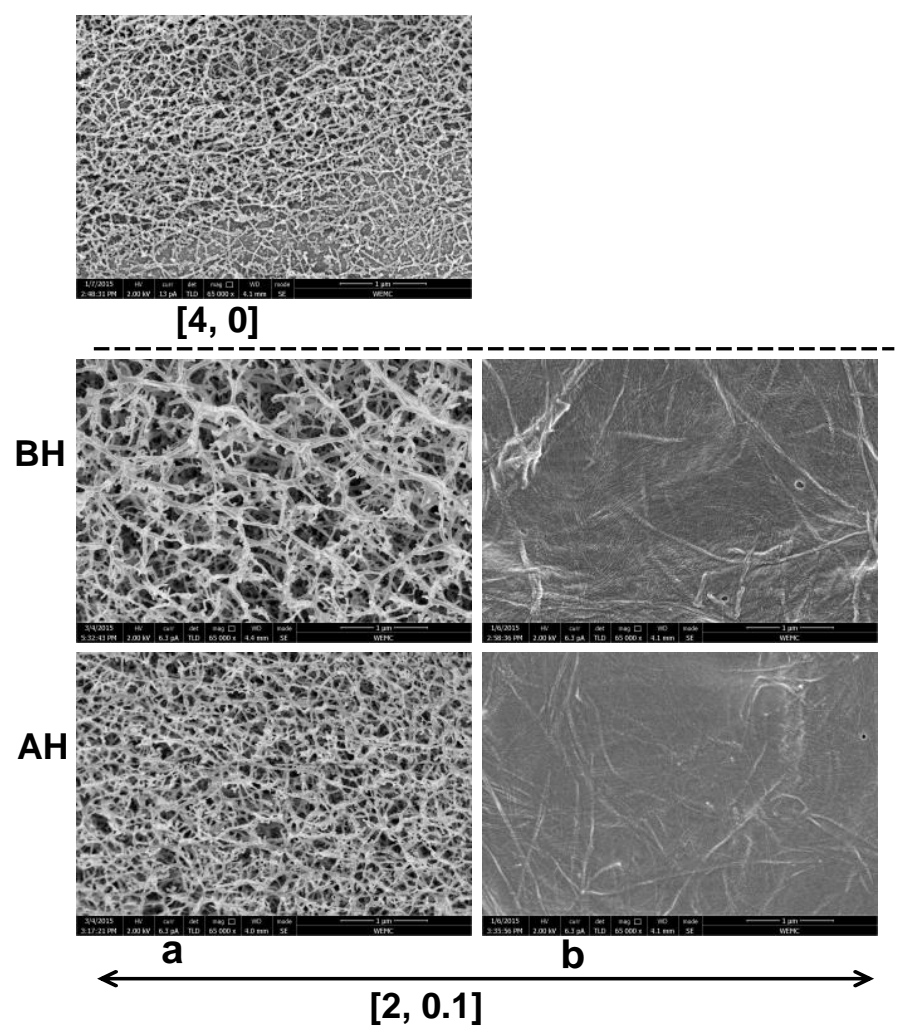

Figure 16 Cryo-SEM (a) and samples treated by air-dry SEM (b) images of mixtures prepared by mixing BC microfibrils before heating and after heating. Top line shows the SEM image of 4 wt\% WPI fibrils after being heated alone. Scale bar corresponds to $1 \mu \mathrm{m}$. The concentration of the mixture is: $[\mathrm{WPI}, \mathrm{BC}]=[2,0.1]$.

In conclusion, the images from TEM, CLSM and SEM do not reveal significant differences in the structure of the WPI fibrils formed alone or in the presence of BC microfibrils. In addition, no difference can be concluded on BC microfibril structure after prolonged heating in the presence of WPI versus BC microfibrils alone. The samples overall showed two independently formed networks consisting of WPI fibrils and BC microfibrils homogeneously present together.

Although the microstructures did not reveal significant difference on the BC microfibril structure after prolonged heating and in the presence of WPI, it is still possible that the length scale of the images might be too small to give distinct results. This is because the 
dispersion of $\mathrm{BC}$ microfibrils is heterogeneous consisting of microfibril bundles, flocs and voids. Therefore, one cannot exclude the possibility that the change on its structure after prolonged heating and in the presence of WPI might be present at smaller or larger length scales than the ones studied. For example, the floc sizes or bundle sizes of the microfibrils might be decreased macroscopically, while microscopically, one might observe the same structure. We like to note that, in contrast to the native mixtures, the viscosity plateau and hysteresis are absent in the flow curves of the mixtures after heating. It is possible that the floc sizes of $B C$ are decreased below a certain value, making them too small to influence the flow curve. As discussed by de Kort et al. ${ }^{52}$, floc sizes of BC microfibrils become smaller by adding CMC. However in their case the reduction in floc size was possibly not large enough to influence the flow curve.

\subsection{Conclusion}

Our results showed that WPI and $\mathrm{BC}$ mcirofibrils form stable dispersions at $\mathrm{pH} 2$, when the concentration of $B C$ is $0.2 \mathrm{wt} \%$ microfibrils and above. The viscosity of the samples is dominated by the presence of $\mathrm{BC}$ microfibrils. The flow curves exhibit two shear-thinning regions and a viscosity plateau. Hysteresis is also observed upon reversing the shear rate. After prolonged heating, the mixtures show the same macroscopic stability as the native ones. The pure $B C$ microfibrils show the same viscosity behaviour as the native pure $B C$ microfibril dispersion. However, in the presence of WPI, the viscosity plateau and the hysteresis are absent in the flow curves of the mixtures. The value of the viscosity is, however, still controlled mainly by the presence of BC microfibrils. The WPI fibrils formed in the presence of $B C$ microfibrils are shorter than the WPI fibrils formed alone. BC microfibrils themselves did not show significant microstructural differences after prolonged heating, and in the presence of WPI. The overall picture of the mixtures that emerges is two independently formed networks composed of WPI fibrils and BC microfibrils that are on a macrocopic scale homogeneous.

\subsection{Acknowledgement}

This work is supported by NanoNextNL, a micro and nanotechnology consortium of the Government of the Netherlands and 130 partners. We thank Tiny Franssen-Verheijen for SEM analysis and Harry Baptist for TEM analysis. 


\subsection{References}

1. C. M. Dobson, Nature, 2003, 426, 884-890.

2. G. W. Smithers, International dairy journal, 2008, 18, 695-704.

3. C. Akkermans, A. J. Van der Goot, P. Venema, E. Van der Linden and R. M. Boom, International dairy journal, 2008, 18, 1034-1042.

4. A. Kroes-Nijboer, P. Venema and E. van der Linden, Food \& function, 2012, 3, 221-227.

5. F. G. Pearce, S. H. Mackintosh and J. A. Gerrard, Journal of Agricultural and Food Chemistry, 2007, 55, 318-322.

6. P. T. Lansbury and H. A. Lashuel, 2006, 443, 774-779.

7. J. D. Sipe and A. S. Cohen, Journal of Structural Biology, 2000, 130, 88-98.

8. V. N. Uversky and A. L. Fink, Biochimica et Biophysica Acta (BBA) - Proteins and Proteomics, 2004, 1698, 131-153.

9. E. Gazit, Febs Journal, 2007, 274, 317-322.

10. Y. D. Livney, Current Opinion in Colloid \& Interface Science, 2010, 15, 73-83.

11. S. Zhang, Nature biotechnology, 2003, 21, 1171-1178.

12. S. G. Bolder, H. Hendrickx, L. Sagis and E. Van der Linden, Applied rheology, 2006, 16, 258-264.

13. S. Bolisetty, L. Harnau, J.-m. Jung and R. Mezzenga, Biomacromolecules, 2012, 13, 3241-3252.

14. S. M. Loveday, M. A. Rao, L. K. Creamer and H. Singh, Journal of Food Science, 2009, 74, R47R55.

15. J. Peng, A. Kroes-Nijboer, P. Venema and E. van der Linden, Soft Matter, 2016, 12, 3514-3526.

16. J. Peng, J. R. Simon, P. Venema and E. van der Linden, Langmuir, 2016, 32, 2164-2174.

17. C. Akkermans, P. Venema, A. J. van der Goot, H. Gruppen, E. J. Bakx, R. M. Boom and E. van der Linden, Biomacromolecules, 2008, 9, 1474-1479.

18. S. G. Bolder, A. J. Vasbinder, L. M. C. Sagis and E. van der Linden, International dairy journal, 2007, 17, 846-853.

19. S. M. Loveday, X. L. Wang, M. A. Rao, S. G. Anema and H. Singh, Food Hydrocolloids, 2012, 27, 242-249.

20. C. Akkermans, A. J. van der Goot, P. Venema, E. van der Linden and R. M. Boom, Food Hydrocolloids, 2008, 22, 1315-1325.

21. S. G. Bolder, L. M. C. Sagis, P. Venema and E. van der Linden, Journal of Agricultural and Food Chemistry, 2007, 55, 5661-5669.

22. C. M. Dobson, Philosophical Transactions of the Royal Society of London Series B-Biological Sciences, 2001, 356, 133-145.

23. J. K. Raynes, J. A. Carver, S. L. Gras and J. A. Gerrard, Trends in Food Science \& Technology, 2014, 37, 42-50.

24. M. Greger, International Journal of Food Safety, Nutrition and Public Health, 2008, 1, 103-115. 
25. S. Moayedzadeh and A. Madadlou, Trends in Food Science \& Technology, 2015, 45, 50-59.

26. L. Bateman, A. Ye and H. Singh, Journal of Agricultural and Food Chemistry, 2010, 58, 98009808.

27. L. Bateman, A. Ye and H. Singh, Journal of Agricultural and Food Chemistry, 2011, 59, 96059611.

28. U. Shimanovich, I. Efimov, T. O. Mason, P. Flagmeier, A. K. Buell, A. Gedanken, S. Linse, K. S. Åkerfeldt, C. M. Dobson and D. A. Weitz, ACS nano, 2015, 9, 43-51.

29. M. Lassé, D. Ulluwishewa, J. Healy, D. Thompson, A. Miller, N. Roy, K. Chitcholtan and J. A. Gerrard, Food Chemistry, 2016, 192, 491-498.

30. R. Jonas and L. F. Farah, Polymer Degradation and Stability, 1998, 59, 101-106.

31. Z. Shi, Y. Zhang, G. O. Phillips and G. Yang, Food Hydrocolloids, 2014, 35, 539-545.

32. A. Svensson, E. Nicklasson, T. Harrah, B. Panilaitis, D. L. Kaplan, M. Brittberg and P. Gatenholm, Biomaterials, 2005, 26, 419-431.

33. M. Iguchi, S. Yamanaka and A. Budhiono, Journal of Materials Science, 35, 261-270.

34. B. V. Mohite and S. V. Patil, Biotechnology and Applied Biochemistry, 2014, 61, 101-110.

35. A. Okiyama, M. Motoki and S. Yamanaka, Food Hydrocolloids, 1993, 6, 503-511.

36. H. Ougiya, K. Watanabe, Y. Morinaga and F. Yoshinaga, Bioscience, Biotechnology, and Biochemistry, 1997, 61, 1541-1545.

37. P. Paximada, A. A. Koutinas, E. Scholten and I. G. Mandala, Food Hydrocolloids, 2016, 54, 245254.

38. P. Paximada, E. Tsouko, N. Kopsahelis, A. A. Koutinas and I. Mandala, Food Hydrocolloids, 2016, 53, 225-232.

39. S.-Y. Yu and K.-W. Lin, Journal of Food Science, 2014, 79, C1117-C1122.

40. H. Zhu, S. Jia, H. Yang, W. Tang, Y. Jia and Z. Tan, Food Science and Biotechnology, 2010, 19, 1479-1484.

41. A. Okiyama, M. Motoki and S. Yamanaka, Food Hydrocolloids, 1992, 6, 479-487.

42. K. W. Lin and H. Y. Lin, Journal of Food Science, 2004, 69, SNQ107-SNQ111.

43. S.-B. Lin, L.-C. Chen and H.-H. Chen, Journal of Food Process Engineering, 2011, 34, 1363-1379.

44. M. Phisalaphong and N. Chiaoprakobkij, Bacterial Cellulose: A Sophisticated Multifunctional Material, 2012, 9, 143-156.

45. R. Mezzenga, J.-M. Jung and J. Adamcik, Langmuir, 2010, 26, 10401-10405.

46. J.-M. Jung and R. Mezzenga, Langmuir, 2009, 26, 504-514.

47. L. M. C. Sagis, C. Veerman and E. van der Linden, Langmuir, 2004, 20, 924-927.

48. A. Kuijk, R. Koppert, P. Versluis, G. van Dalen, C. Remijn, J. Hazekamp, J. Nijsse and K. P. Velikov, Langmuir, 2013, 29, 14356-14360.

49. S. J. Veen, A. Kuijk, P. Versluis, H. Husken and K. P. Velikov, Langmuir, 2014, 30, 13362-13368.

50. S. J. Veen, P. Versluis, A. Kuijk and K. P. Velikov, Soft Matter, 2015, 11, 8907-8912. 
Rheology and microstructure of dispersions of protein fibrils and cellulose microfibrils

51. S. G. Bolder, H. Hendrickx, L. M. C. Sagis and E. van der Linden, Journal of Agricultural and Food Chemistry, 2006, 54, 4229-4234.

52. D. de Kort, S. J. Veen, H. van As, D. Bonn, K. P. Velikov and J. van Duynhoven, Soft Matter, 2016, 12, 4739-4744.

53. A. Okiyama, M. Motoki and S. Yamanaka, Food Hydrocolloids, 1993, 6, 493-501.

54. M. lotti, $\varnothing$. W. Gregersen, S. Moe and M. Lenes, Journal of Polymers and the Environment, 2010, 19, 137-145.

55. A. Karppinen, T. Saarinen, J. Salmela, A. Laukkanen, M. Nuopponen and J. Seppälä, Cellulose, 2012, 19, 1807-1819.

56. F. Martoïa, C. Perge, P. J. Dumont, L. Orgéas, M.-A. Fardin, S. Manneville and M. N. Belgacem, Soft Matter, 2015, 11, 4742-4755.

57. O. Nechyporchuk, M. N. Belgacem and F. Pignon, Carbohydrate Polymers, 2014, 112, 432-439.

58. E. Saarikoski, T. Saarinen, J. Salmela and J. Seppälä, Cellulose, 2012, 19, 647-659.

59. T. Saarinen, M. Lille and J. Seppälä, Annu Trans Nord Rheol Soc, 2009, 17, 121-128. 
Chapter 3 


\section{Chapter 4}

Duplex gels from whey protein isolate and cellulose microfibrils

Submitted as:

Jinfeng Peng, Vincenzo Calabrese, William Nicholas Ainis, Ruben Scager, Krassimir P. Velikov, Paul Venema, Erik van der Linden. Duplex gels from whey protein isolate and cellulose microfibrils. 


\begin{abstract}
We investigated the heat-induced gelation of whey protein isolate (WPI) in the presence of bacterial cellulose (BC) microfibrils at $\mathrm{pH} 7$ at different concentrations of $\mathrm{NaCl}$. The small and large deformation rheological properties were characterized. The microstructure of the gels was studied using confocal laser scanning microscopy and scanning electron microscopy. The water holding capacity of the WPI-BC microfibril gels was also determined. Our results showed that WPI and BC microfibrils form a homogeneous dispersion at $\mathrm{pH}$ 7. Upon heating, the WPI gel was formed independently in the presence of the $\mathrm{BC}$ microfibril gel, resulting in the formation of a duplex gel. The gel structure and gelation dynamics of WPI was not influenced by the presence of $B C$ microfibrils. However, the presence of $B C$ microfibrils did increase the storage modulus of the WPI gel, with an increase being negligible when the strength of the WPI gel is above a certain value. With an increase of $\mathrm{NaCl}$ concentration, the WPI gel structure changes from fine-stranded to a particulate gel, while the BC microfibril gel structure remains unchanged. No macroscopic phase separation could be observed in the WPI-BC microfibril gels. Our results showed that the rheological properties and water holding capacity of the WPI-BC microfibril duplex gels are mainly dominated by the WPI and that BC microfibrils can be added to tune the original properties of the WPI gel.
\end{abstract}




\subsection{Introduction}

Proteins and polysaccharides are common structuring components in foods. They are normally present together due to their functionalities as gelling agents, thickening agents and stabilizers in foods. These functionalities are important in defining the structure, physio-chemical and mechanical properties of food products. The functionality of these two components is largely dependent on their interactions under different conditions such as concentration, $\mathrm{pH}$, temperature and ionic strength. ${ }^{1}$ When mixing a protein with a polysaccharide, they might be co-soluble, incompatible or form a complex. Incompatibility is often observed leading to macroscopic phase separation. ${ }^{1-3}$ Interactions between proteins and polysaccharides have been studied and reviewed extensively in literature. ${ }^{1-7}$

WPI is a common food ingredient and has received much interest in literature due to its various well-known functional and nutritional properties. ${ }^{8}$ In particular, gelation, an important functional property of whey proteins, has received extensive attention due to its ability to form different gel structures ranging from fine-stranded to particulate gels when appropriate conditions are applied. ${ }^{9}$ By incorporating polysaccharides, the gelation of WPI can be influenced, allowing for tuning the final structure and properties of the gel. This also leads to a wide range of opportunities for designing novel structures and various functionalities for specific applications. On the other hand, challenges like the high cost of WPI is a major concern in food industry. ${ }^{10}$ Many polysaccharides have been added to WPI to influence the functionality and microstructure. Examples of polysaccharides that were

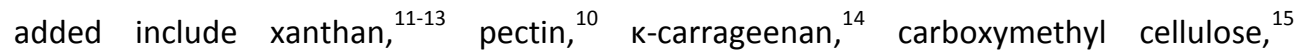
galactomannan, ${ }^{16}$ and konjac glucomannan. ${ }^{17,18}$

As one of the most abundant biopolymers in nature, cellulose microfibrils have gained much attention as a key source for sustainable and natural materials. ${ }^{19}$ In particular, microfibrils from bacterial cellulose (BC), has received increased attention in food fields due to its multi-functional potential uses, such as a thickening, gelling, emulsifying, stabilizing, texturizer and water-binding agent. ${ }^{20-26} \mathrm{BC}$ already finds its presence in food, such as the well-known dessert in the Philippines, named Nata de Coco, ${ }^{27}$ which has gained increasing popularity worldwide. ${ }^{20} \mathrm{BC}$ is a dietary fibre and therefore is interesting to be used in products from a health point of view, and it can be used as a structuring agent. ${ }^{28-30}$ The BC microfibrils are of high purity compared to that of plant cellulose and 
contain only hydroxyl groups as functional groups. ${ }^{31}$ The microfibrils in $\mathrm{BC}$ have a ribbonlike structure, having a width in the order of nanometers and a length in the order of micrometers. The microfibrils tend to agglomerate into space-filling networks in water, leading to a gel-like structure. Recently, colloidal dispersions of BC microfibrils were prepared from commercial nata de coco through an high-energy deagglomeration process. $^{32}$ Moreover, addition of carboxymethyl cellulose (CMC) in the BC microfibril dispersions during high-energy de-agglomeration treatment was reported to be able to form a more homogeneous dispersion, presumably due to the adsorption of $\mathrm{CMC}$ on the surface of BC microfibrils. ${ }^{33,34}$

Despite numerous studies in literature ${ }^{31,35,36}$ and its broad potential applications in foods, ${ }^{37}$ there is only limited research focusing on the interaction between WPI and BC microfibrils under different conditions. In this study, we investigated the heat-induced biopolymer gels prepared from WPI and $\mathrm{BC}$ microfibrils at $\mathrm{pH} 7$, at different $\mathrm{NaCl}$ concentrations. The rheological properties of the resulting gels were studied under small and large deformation, and the microstructure and water holding capacity were characterized.

\subsection{Materials and Methods}

\subsubsection{Preparation of whey protein isolate (WPI) solution}

The WPI stock solution is prepared following the same procedure as described in Chapter 2. A 25 wt\% WPI stock solution was prepared by dissolving the WPI (Bipro, Davisco, lot \# JE 198 -1-420, USA) powder in MilliQ water. The solution was stirred at $4{ }^{\circ} \mathrm{C}$ for 2 days to ensure complete hydration. The $\mathrm{pH}$ of the WPI stock solution was adjusted to $\mathrm{pH} 7 \mathrm{using}$ a $3 \mathrm{M} \mathrm{NaOH}$ solution. To remove undissolved protein, the solution was filtered using a filter (Hydrophilic PES $0.45 \mu \mathrm{m}$, Millipore Millex-HP). An UV spectrophotometer (Cary 50 Bio, Varian) was used to determine the protein concentration of the solution at a wavelength of $280 \mathrm{~nm}$, and a calibration curve determined from WPI solutions at known concentrations. The stock solution was stored at $4{ }^{\circ} \mathrm{C}$ in the fridge for further use. 


\subsubsection{Preparation of bacterial cellulose (BC) microfibril dispersion}

The dispersion of BC microfibrils was kindly provided by Unilever R\&D Vlaardingen, the Netherlands. The obtained dispersion was prepared in the same way as used in chapter 2 . Details of the preparation procedure were described previously. ${ }^{32,33}$ The concentration of the stock dispersion used for sample preparation is $1 \mathrm{wt} \%$.

\subsubsection{Preparation of WPI-BC microfibril gels}

The 25 wt\% WPI stock solution and 1 wt\% BC microfibril dispersion were used to prepare the mixtures. The mixtures were prepared by adding BC microfibrils to the WPI solution using a plastic pipette with a cut-off tip. All mixtures had the same final WPI concentration of $13 \mathrm{wt} \%$ and the $\mathrm{BC}$ concentrations varied from $0,0.05,0.1,0.2$, and 0.3 to $0.4 \mathrm{wt} \%$. The $\mathrm{pH}$ of the mixtures was adjusted to $\mathrm{pH} 7$ using a $3 \mathrm{M} \mathrm{NaOH}$ solution. The mixtures were stirred for $30 \mathrm{~min}$ to ensure a homogeneous mixing. Subsequently, the mixtures were heated in a water bath (Haake Phoenix II C25P) for gel preparation. The heating procedure consisted of first increasing temperature from 20 to $80{ }^{\circ} \mathrm{C}$ within 30 min (heating rate of $2{ }^{\circ} \mathrm{C} / \mathrm{min}$ ), holding at $80{ }^{\circ} \mathrm{C}$ for $30 \mathrm{~min}$ and then cooling from 80 to 20 ${ }^{\circ} \mathrm{C}$ within $30 \mathrm{~min}$. The gels were stored at room temperature $\left(20^{\circ} \mathrm{C}\right)$ for further analysis.

Mixtures containing 13 wt\% WPI and $0,0.1$ or 0.3 wt\% BC microfibrils, were chosen to study the effect of $\mathrm{NaCl}$ on the gels. The $\mathrm{NaCl}$ solution was added after mixing WPI and $\mathrm{BC}$ microfibrils together. The final $\mathrm{NaCl}$ concentrations in the mixtures were 20, 50, 100, 200 and $250 \mathrm{mM}$, adjusted by using a stock $\mathrm{NaCl}$ solution of $3 \mathrm{M}$. All samples were mildly stirred for at least 30 minutes to provide homogeneous samples. The procedure for gel preparation is the same as described above.

\subsubsection{Differential Scanning Calorimetry (DSC)}

Prior to the measurement, about $50 \mathrm{mg}$ sample was weighted in a sealed stainless steel pan. Subsequently, the samples placed in the pan were transferred into the equipment (Diamond series DSC, Perkin Elmer, Pyris, USA). Measurements started with an equilibration at $20^{\circ} \mathrm{C}$ for $2 \mathrm{~min}$. The samples were then heated from $20^{\circ} \mathrm{C}$ to $100{ }^{\circ} \mathrm{C}$ at a heating rate of $10^{\circ} \mathrm{C} / \mathrm{min}$, held at $100{ }^{\circ} \mathrm{C}$ for $1 \mathrm{~min}$, and cooled from $100^{\circ} \mathrm{C}$ to $20^{\circ} \mathrm{C}$ at a cooling rate of $10^{\circ} \mathrm{C} / \mathrm{min}$. A second heating step from $20^{\circ} \mathrm{C}$ to $100^{\circ} \mathrm{C}$ at a heating rate of 
$10^{\circ} \mathrm{C} / \mathrm{min}$ was applied after an equilibration at $20^{\circ} \mathrm{C}$ for $2 \mathrm{~min}$. Measurements were performed in duplicate. We used the software of Pyris (Perkin Elmer) for data analysis.

\subsubsection{Small deformation rheology}

Small deformation rheological properties of the WPI-BC microfibril mixtures during gel formation were monitored on a stress-controlled rheometer (MCR 302, Anton Paar) using a sandblasted concentric cylinder geometry (CC17/TI/S-SN38492). Sample transfer to the rheometer was performed using a plastic pipette with a cut-of tip to minimize the $B C$ microfibril alignment. A solvent trap was used and the sample was covered with a thin layer of paraffin oil to prevent evaporation. Storage modulus $G^{\prime}$ and loss modulus $G^{\prime \prime}$ were measured at a frequency of $1 \mathrm{~Hz}$ and a strain of $0.1 \%$ during gel formation by increasing temperature from 20 to $80^{\circ} \mathrm{C}$ at a heating rate of $2^{\circ} \mathrm{C} / \mathrm{min}$, holding temperature at $80^{\circ} \mathrm{C}$ for $30 \mathrm{~min}$, cooling from 80 to $20^{\circ} \mathrm{C}$ at a cooling rate of $2^{\circ} \mathrm{C} / \mathrm{min}$ and then holding the temperature at $20^{\circ} \mathrm{C}$ for $30 \mathrm{~min}$. Subsequently, a frequency sweep was carried out at a strain of $0.1 \%$ and the frequency being increased from 0.01 to $100 \mathrm{~Hz}$ within $30 \mathrm{~min}$ at 20 ${ }^{\circ} \mathrm{C}$. In addition, a strain sweep at a frequency of $1 \mathrm{~Hz}$ was performed by increasing the strain from 0.01 to $1000 \%$ within $30 \mathrm{~min}$ at $20^{\circ} \mathrm{C}$ in order to determine the linear viscoelastic region. The ratio of $G^{\prime \prime} / G^{\prime}$ is indicated as tangent $\delta$. Samples containing $0 w t \%$ WPI and 0.3 wt\% BC, 13 wt\% WPI and 0.3 wt\% BC, 13 wt\% WPI and 0.4 wt\% BC were measured in triplicate.

\subsubsection{Scanning Electron Microscopy (SEM)}

Gels for SEM analysis were prepared in $20 \mathrm{ml}$ syringes (BD plastipak, Spain) following the same heating procedure as described above. Each of the WPI-BC mirofibril gels was cut into 5 pieces of $1 \mathrm{~cm}$ in diameter and $1.3 \mathrm{~cm}$ in height and then, these gel pieces were placed in $2.5 \%(\mathrm{v} / \mathrm{v})$ glutaraldehyde solution for $8 \mathrm{hrs}$ in order to crosslink the proteins. After crosslinking, the excess glutaraldehyde was removed by placing the gel pieces in MilliQ water while stirring overnight. The water was replaced gradually by acetone in 5 steps: starting from 10, 30, 50, and, 70 to $100 \%$ acetone. During each step, the samples were stirred for 1 hour. The samples were then left in $100 \%$ acetone. Subsequently, the sample pieces were dried by critical point drying (CPD 300, Leica, Vienna, Austria). After drying, the samples were fractured and attached to a SEM sample holder using Carbon 
Conductive Cement (Leit-C, Neubauer Chemicalien, Germany). In order to remove all the solvent from the adhesive, the samples were stored overnight under vacuum. After sputter coating with a $10 \mathrm{~nm}$ thick layer of iridium (SCD 500, Leica, Vienna, Austria) the fractured surfaces were analyzed at $2 \mathrm{kV}$ in a field emission scanning electron microscope (Magellan 400, FEI, Eindhoven, the Netherlands).

\subsubsection{Confocal laser scanning microscopy (CLSM)}

$5 \mu \mathrm{l}$ of $0.2 \%$ Rhodamine $\mathrm{B}$ and $10 \mu \mathrm{l}$ of $0.05 \%$ Calcofluor White were added to stain the WPI and $B C$, respectively, in a total sample volume of $1 \mathrm{ml}$. After adding the dye, the sample was mixed for $10 \mathrm{~s}$ on a vortex. $125 \mu \mathrm{l}$ of the sample was transferred into CLSM cuvettes (Gene Frame $125 \mu \mathrm{l}$, Thermo Scientific), after which were sealed with a cover glass (Menzel-glaser, Thermo Scientific, Germany). For gel formation, the samples were heated in a water bath (Haake Phoenix II C25P) with the temperature increasing from 20 to $80^{\circ} \mathrm{C}$ within $30 \mathrm{~min}$, holding at $80^{\circ} \mathrm{C}$ for $30 \mathrm{~min}$ and decreasing from 80 to $20^{\circ} \mathrm{C}$ within $30 \mathrm{~min}$. The samples were kept at room temperature for one day prior to image analysis. CLSM images were taken using a Zeiss LSM 510 META microscope (Zeiss, Germany) equipped with an Axiovert 200M inverted microscope. The oil immersion objective (PlanApochromat $63 x / 1.4$ oil DIC) was used. The excitation and emission wavelength of Rhodamine B are $543 \mathrm{~nm}$ and $560 \mathrm{~nm}$. The excitation and emission wavelength of Calcofluor White are $405 \mathrm{~nm}$ and $420 \mathrm{~nm}$.

\subsubsection{Large deformation rheology}

Gels were prepared in pre-lubricated (paraffin oil) $20 \mathrm{ml}$ syringes (BD plastipak, Spain) and cut into cylinders of $20 \mathrm{~mm}$ in height and $20 \mathrm{~mm}$ in diameter using a wire cutter. To measure their fracture properties, an uniaxial compression test was performed using a Texture Analyser (TA-XT plus, Stable Micro Systems Itd., Godalming U.K) mounted with a $50 \mathrm{~kg}$ load cell and a cylindrical plastic probe of $75 \mathrm{~mm}$ in diameter. Paraffin oil was applied on both the top and bottom of the gel to prevent friction during compression. The gels were compressed to $80 \%$ of their initial height between two parallel plates at a constant deformation rate of $1 \mathrm{~mm} / \mathrm{s}$ and a post-test speed of $10 \mathrm{~mm} / \mathrm{s}$. Each sample was measured four times at room temperature. We calculated the true stress, true strain and Young's modulus as described elsewhere. ${ }^{38}$ 


\subsubsection{Water holding capacity (WHC)}

For the WHC measurement of the gels, we used a method described previously. ${ }^{38} \mathrm{~A}$ microcentrifuge filtration unit containing an inner spin tube and a $2 \mathrm{ml}$ Eppendorf tube (Axygen Biosciences, Inc., Union City, USA) was used. The gels were cut into cylinders of 10 $\mathrm{mm}$ in height and $4.8 \mathrm{~mm}$ in diameter using a cork borer and placed carefully at the bottom of the spin tube. Two pieces of filter paper (Macherey-Nagel MN 85/70 BF, $100 \mathrm{~mm}$, Germany) with a diameter of $5 \mathrm{~mm}$ were placed on the spin tube to reduce grid size and avoid the leakage of the gels in the supernatant. Samples were centrifuged at $3350 \mathrm{~g}$ for $15 \mathrm{~min}$ at $20^{\circ} \mathrm{C}$. The measurements were performed in duplicate. The water removed from the gel was collected at the bottom of the Eppendorf tube. The WHC of the gels is calculated as the remaining water (\%) in the gel after centrifugation according to:

$$
W H C=\frac{W_{t}-\left(W_{g}+W_{f p}\right)}{W_{t}} * 100(\%)
$$

Where $W H C$ is the water holding capacity (\%), $W_{t}$ is the amount of water present in the sample (g), $W_{g}$ is the removed water from the sample at a given centrifugal force $(\mathrm{g}) . W_{f p}$ is the water entrapped in the filter papers.

\subsection{Results and discussion}

\subsubsection{Macroscopic images}

The images of the WPI-BC microfibril mixtures both before and after gel formation (Figure 1) showed that the samples are homogeneously mixed and form a stable single-phase system, while the addition of $B C$ microfibrils increased the turbidity of the mixture. Previous studies reported that dispersion of BC microfibrils prepared by high-energy mechanical de-agglomeration showed a highly heterogeneous network consisting of fibril bundles, flocs, and voids (from ten to hundreds of micrometers) induced by their attractive interactions. ${ }^{32}$ Increasing the $\mathrm{BC}$ microfibril concentration leads to an increase in size of the flocs and decrease of the size of voids, however, the size of the bundles remained constant. ${ }^{32}$ All samples were stable on a macroscopic scale as probed by the images (Figure 1). 


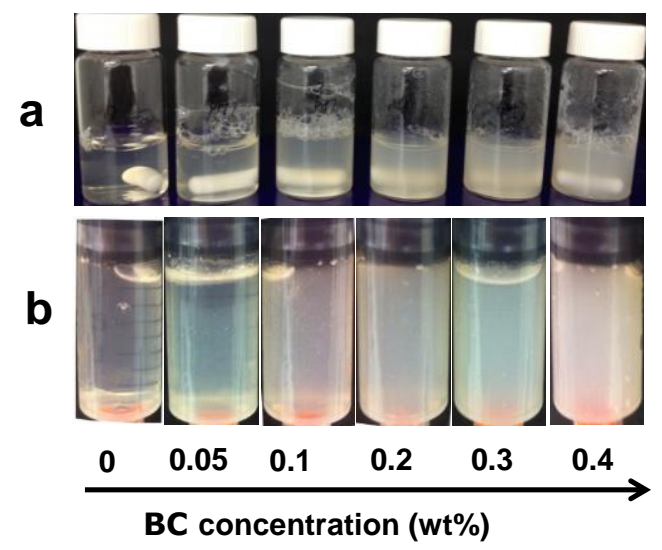

Figure 1 Images of WPI-BC microfibril mixtures (a) and their corresponding heat-induced gels (b) at $\mathrm{pH} 7$, prepared from $13 \mathrm{wt} \% \mathrm{WPI}$ and $\mathrm{BC}$ microfibrils with concentrations of $0,0.05,0.1,0.2,0.3$ or $0.4 \mathrm{wt} \%$. The concentration of BC microfibrils (wt\%) is indicated below each image.

\subsubsection{Differential Scanning Calorimetry (DSC)}

DSC was used to determine the denaturation temperature and the corresponding enthalpy change of the WPI-BC microfibril mixtures in comparison to that of the pure WPI.
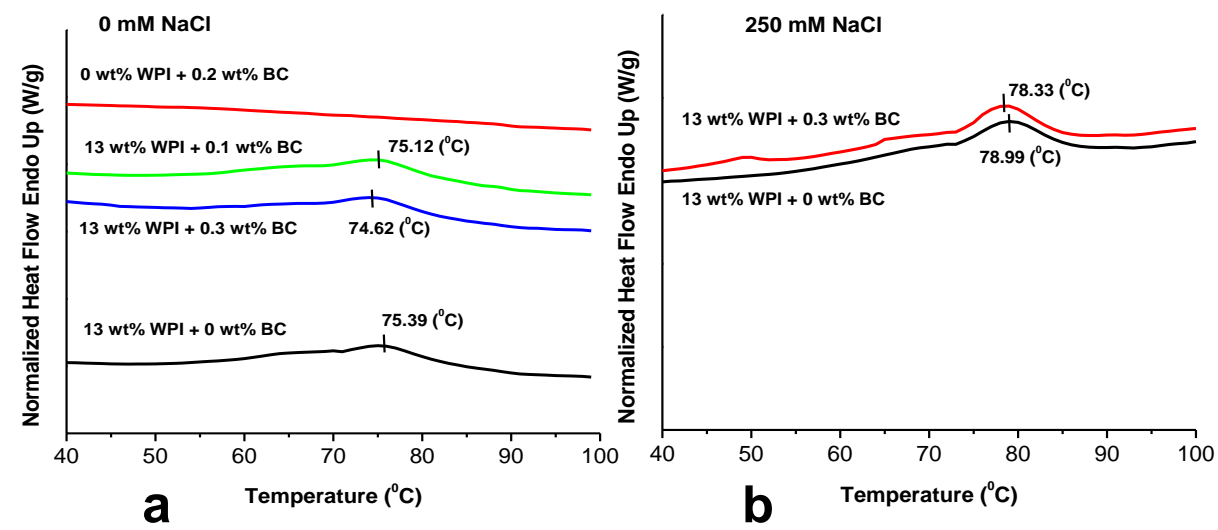

Figure 2 DSC thermograms for pure WPI, pure BC microfibrils and the mixed WPI-BC microfibrils at $\mathrm{NaCl}$ concentrations of $\mathrm{O}(\mathrm{a})$ and $250 \mathrm{mM}$ (b). The concentration of the samples is indicated in the figures. 
For pure BC microfibrils, no distinct endothermic peaks were observed at $\mathrm{pH} 7$ (Figure 2a), confirming that $\mathrm{BC}$ is stable upon heating up to $100^{\circ} \mathrm{C} .{ }^{39}$ For pure WPI, the endothermic peak around $75^{\circ} \mathrm{C}$ is in accordance with the denaturation temperature of WPI. ${ }^{40}$ In the mixture of WPI and BC microfibrils at concentrations of 0.1 and $0.3 \mathrm{wt} \%$, the endothermic peak is similar to that of the pure WPI sample. The denaturation temperature was also not influenced by the presence of $\mathrm{BC}$ microfibrils. This is further shown in samples with $\mathrm{NaCl}$ concentration of $250 \mathrm{mM}$ (Figure 2b), where the mixture showed a similar endotherm peak to that of pure WPI sample. The increase in denaturation temperature of WPI at 250 $\mathrm{mM} \mathrm{NaCl}$ is in agreement with literature. ${ }^{41}$ As $B C$ microfibrils do not show any endothermic peak during heating, and the denaturation temperature of the WPI-BC microfibril mixtures is independent of $B C$ microfibril concentration, no specific interactions exist between WPI and BC microfibrils. ${ }^{42}$

\subsubsection{Rheological properties}

The results of the rheological experiments are shown below. 

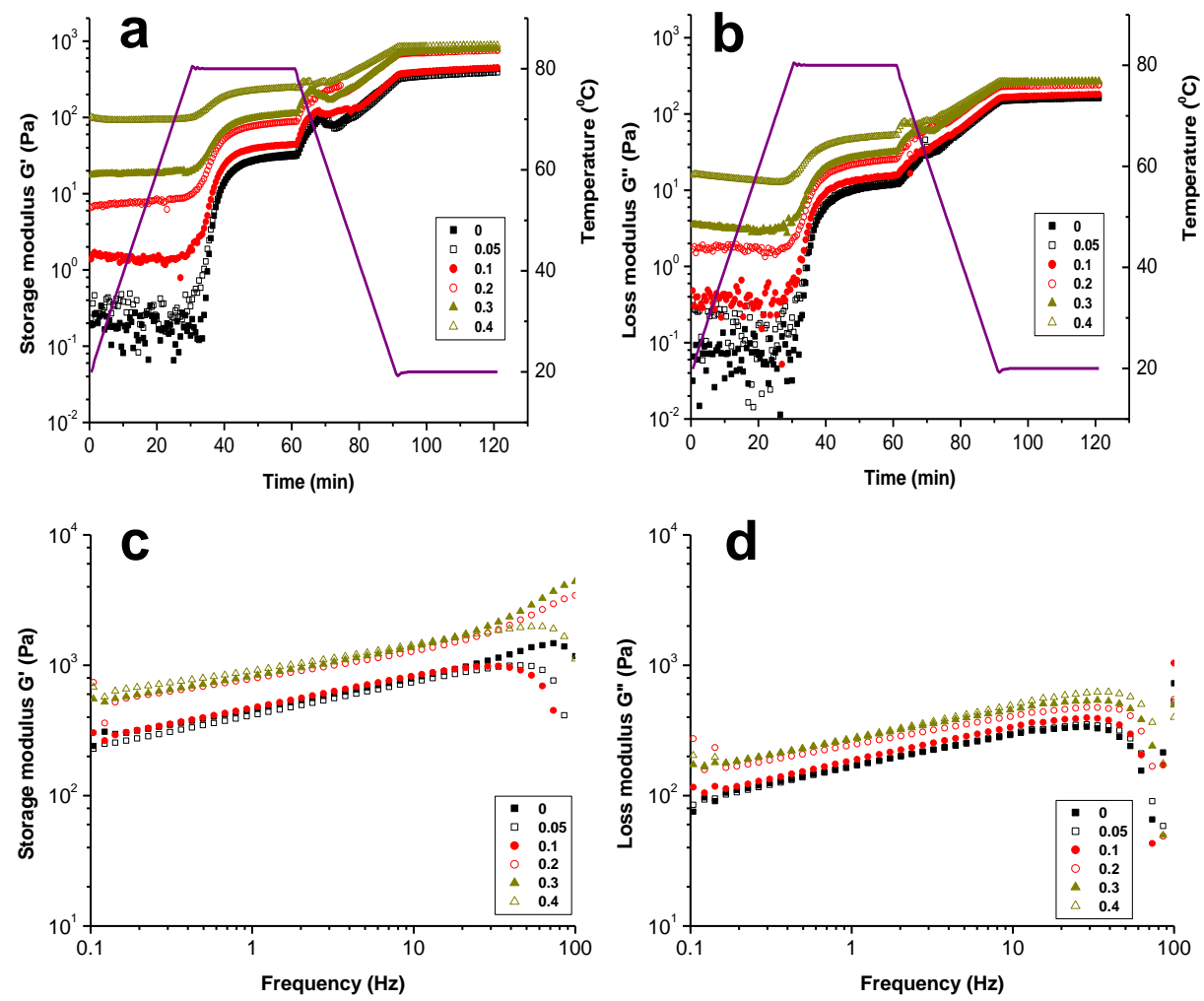

Figure 3 Rheological properties of WPI-BC microfibril gels containing a constant $13 \mathrm{wt} \% \mathrm{WPI}$ and $\mathrm{BC}$ microfibril concentrations at $0,0.05,0.1,0.2,0.3$, and $0.4 \mathrm{wt} \%$. The development of storage modulus G' and the loss modulus G" as a function of time during gel formation are shown in (a) and (b). The temperature profile is indicated by the purple line. Figures (c) and (d) show the storage modulus G' and loss modulus G' as a function of frequency at the strain of $0.1 \%$. 


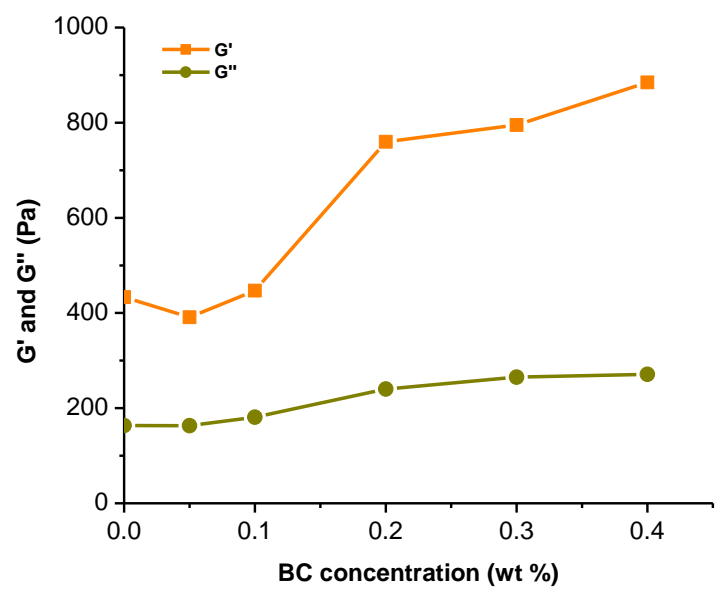

Figure 4 The storage modulus G' and loss modulus G' as a function of BC microfibril concentration. The values of G' and G' are taken from the last point of the measurement during holding the gels at $20^{\circ} \mathrm{C}$ for $30 \mathrm{~min}$.

Figure 3 summarises the findings on the rheological properties of the WPI-BC microfibril gels. The storage modulus $\mathrm{G}^{\prime}$ and loss modulus G" of WPI-BC microfibril gels as a function of $\mathrm{BC}$ microfibril concentration (Figure $3 \mathrm{a}$ and $3 \mathrm{~b}$ ) showed a comparable trend to that for the pure WPI. Both $\mathrm{G}^{\prime}$ and $\mathrm{G}^{\prime \prime}$ of the samples increased upon heating at $80^{\circ} \mathrm{C}$ and reached a plateau with time. Upon cooling from 80 to $20^{\circ} \mathrm{C}$, the $\mathrm{G}^{\prime}$ and $\mathrm{G}^{\prime \prime}$ of gels with $\mathrm{BC}$ microfibrils showed an abrupt decrease, followed by a further increase of the $G^{\prime}$ and $G^{\prime \prime}$ upon further cooling. The abrupt decrease in $\mathrm{G}^{\prime}$ and $\mathrm{G}^{\prime \prime}$ is possibly caused by the creep of the paraffin oil between the gel surface and rheometer cup. ${ }^{43}$ When holding at $20{ }^{\circ} \mathrm{C}$, the $\mathrm{G}^{\prime}$ and $\mathrm{G}^{\prime \prime}$ of all samples remained constant. The $\mathrm{G}^{\prime \prime}$ development of all samples showed a similar trend to that of the $G^{\prime}$ development (Figure 3b). Plotting the $G^{\prime}$ and $G^{\prime \prime}$ value after cooling the gels as a function of BC microfibril concentration in WPI gels (Figure 4), we observed a gradual increase of $G^{\prime}$, reaching approximately 10 times higher $(0.4$ wt\% BC) than the WPI sample without BC microfibrils. This implies that the addition of $B C$ microfibrils to WPI resulted in higher firmness of the gel. In contrast to $\mathrm{G}^{\prime}$, the increase of $G^{\prime \prime}$ with increasing BC microfibril concentration is only a small effect above $0.1 \mathrm{wt} \% \mathrm{BC}$ microfibril. The $G^{\prime}$ and $G^{\prime \prime}$ of all WPI gels with BC microfibrils showed the same linear dependency on frequency as that of the pure WPI gel (Figure $3 c$ and $3 d$ ), giving the same 
slope of the curve. This suggests that the WPI gel dominates the rheological properties in the WPI-BC microfibril gels.

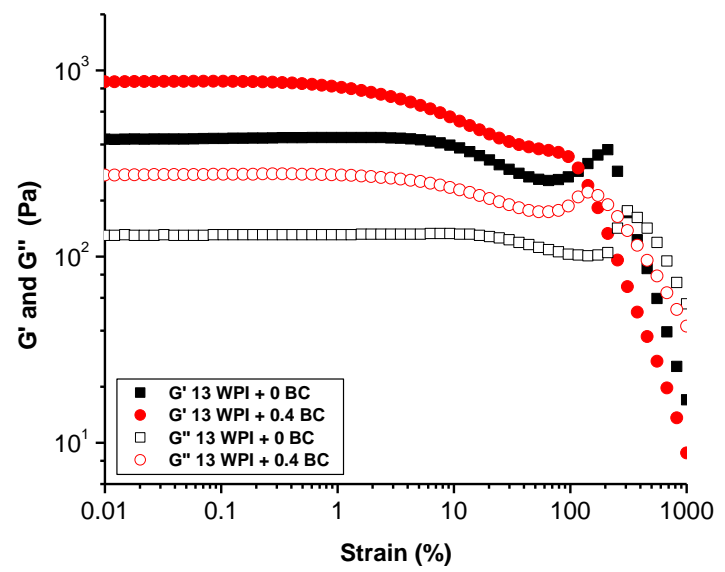

Figure 5 The storage modulus G' and loss modulus G" as a function of strain at frequency of $1 \mathbf{~ H z}$ at $20^{\circ} \mathrm{C}$ for a $13 \mathrm{wt} \%$ pure WPI gel and $13 \mathrm{wt} \%$ WPI gel containing $0.4 \mathrm{wt} \% \mathrm{BC}$ microfibrils.

The pure WPI gels show a linear viscoelastic region (LVR) up to a strain of $10 \%$ (Figure 5). With addition of $0.4 \mathrm{wt} \% \mathrm{BC}$, the LVR of the gels decreased to approximately $1 \%$ strain, suggesting gel breakdown at lower strain. This indicates that the gels become more brittle when $\mathrm{BC}$ microfibrils are added to the WPI gel.

At low ionic strength, WPI forms a fine-stranded gel structure upon heating. Increasing the ionic strength in the WPI solutions screens the intermolecular repulsion, resulting in the formation of a particulate gel structure. ${ }^{44}, 45$ To investigate the effect of $\mathrm{NaCl}$ concentration on heat-induced WPI-BC microfibril gelation, 20, 100 and $250 \mathrm{mM} \mathrm{NaCl}$ were added to the WPI-BC microfibril mixtures prior to heating. The rheological properties are shown below in Figure 6 and Figure 7. 

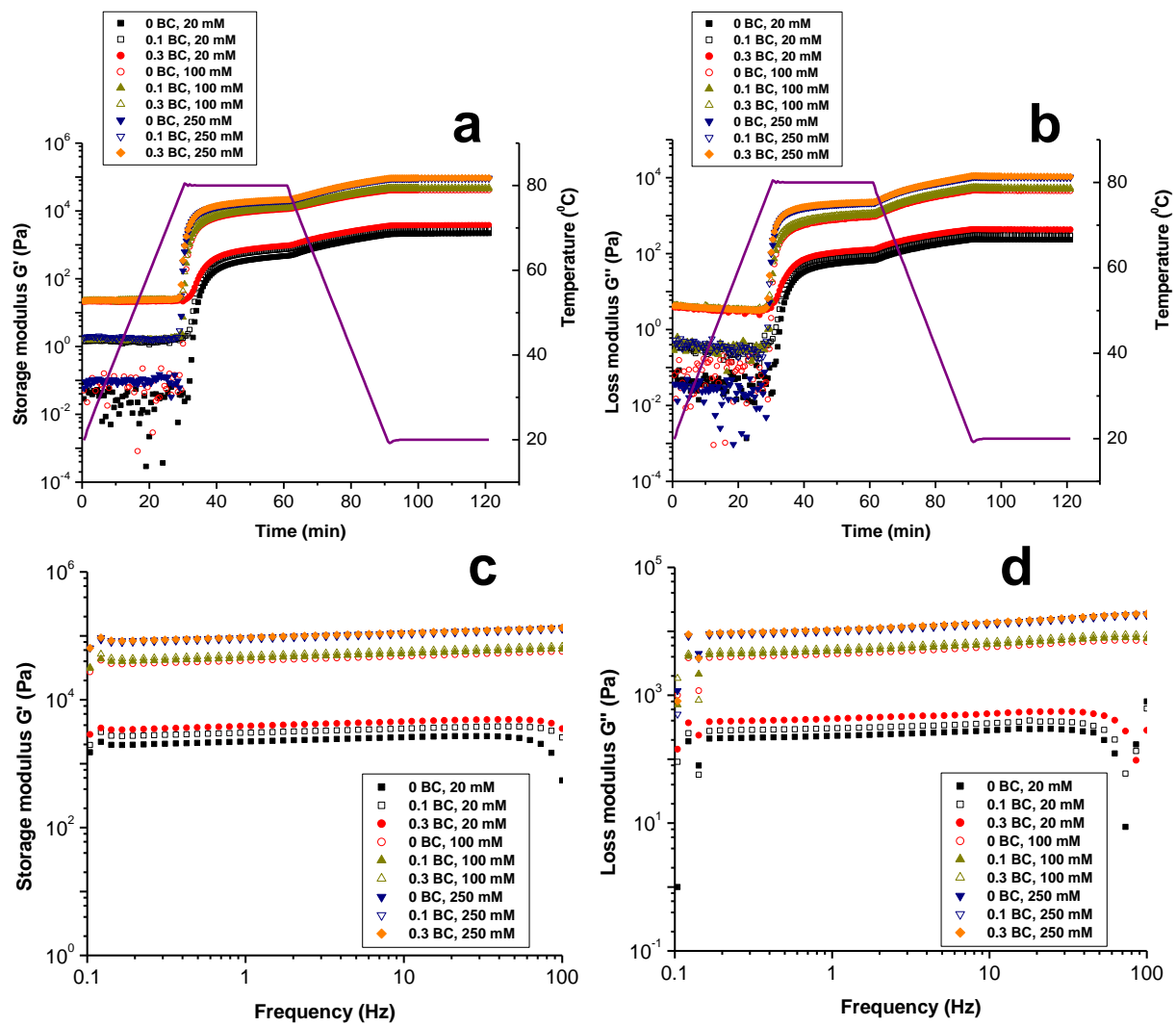

Figure 6 Rheological properties of 13 wt\% WPI gel containing $0,0.1$ and 0.3 wt\% BC microfibrils with $\mathrm{NaCl}$ concentrations of 20, 100, and $250 \mathrm{mM}$. The storage modulus $\mathrm{G}^{\prime}(\mathrm{a})$ and loss modulus $\mathrm{G}^{\prime \prime}$ (b) during gel formation are plotted as a function of time at a strain of $0.1 \%$ and frequency of $1 \mathrm{~Hz}$. The purple line represents the temperature profile. Figures (c) and (d) show the storage modulus $G^{\prime}(c)$ and loss modulus G' (d) of the gels as a function of frequency at strain of $0.1 \%$ at $20^{\circ} \mathrm{C}$. 


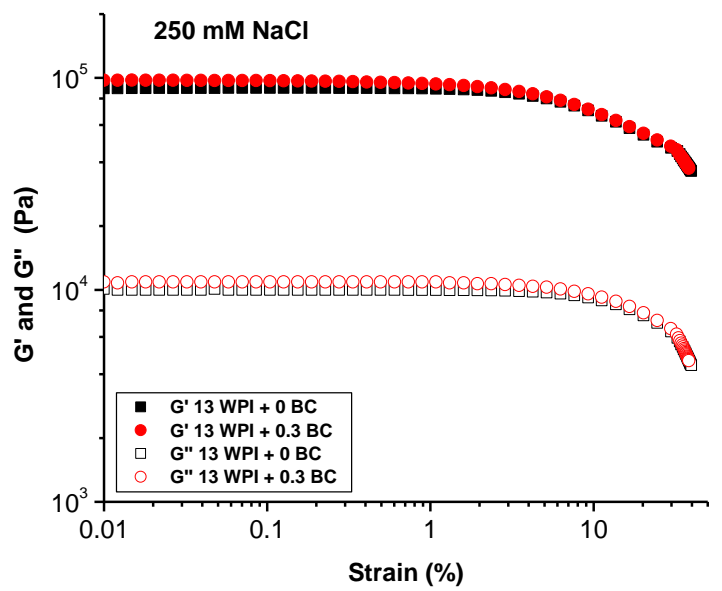

Figure 7 The storage modulus G' and loss modulus G" as a function of strain at a frequency of $\mathbf{1 ~ H z}$ at $20^{\circ} \mathrm{C}$ for pure $13 \mathrm{wt} \% \mathrm{WPI}$ gel and $13 \mathrm{wt} \%$ WPI gel containing $0.3 \mathrm{wt} \% \mathrm{BC}$ microfibrils. The $\mathrm{NaCl}$ concentration of both samples was $250 \mathrm{mM}$.
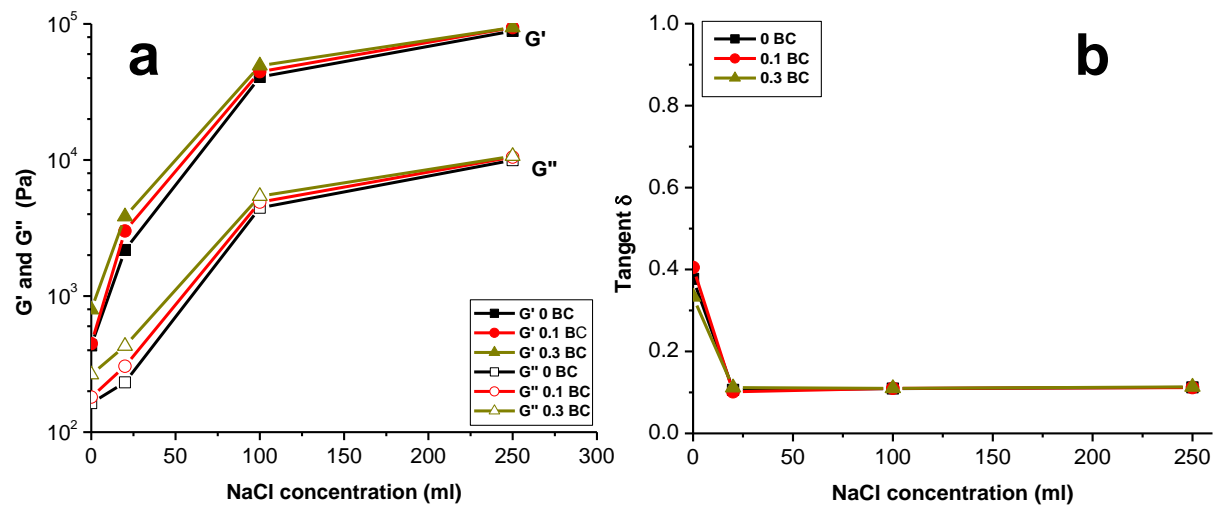

Figure 8 Storage modulus G' (filled symbols) and loss modulus G" (open symbols) (a) and Tangent $\delta$ (b) as a function of $\mathrm{NaCl}$ concentration for 13 wt\% WPI gels containing BC microfibril concentration of $0,0.1$, and $0.3 \mathrm{wt} \%$. The values of $\mathrm{G}^{\prime}$ and $\mathrm{G}^{\prime}$ ' were taken from the last point of holding samples at $20^{\circ} \mathrm{C}$ for $30 \mathrm{~min}$ after gel formation. Lines are used to guide the eye. 
The G' (Figure 6a) and G" (Figure 6b) as a function of time for WPI-BC microfibril gels with $20 \mathrm{mM} \mathrm{NaCl}$ are comparable to that of pure WPI with $20 \mathrm{mM} \mathrm{NaCl}$. The $\mathrm{G}^{\prime}$ and $\mathrm{G}^{\prime \prime}$ showed a sharp increase when temperature reaches $80^{\circ} \mathrm{C}$, followed by further increase upon cooling and holding temperature at $20^{\circ} \mathrm{C}$. Increasing $\mathrm{NaCl}$ to $100 \mathrm{mM}$, we observed a sharper increase in $\mathrm{G}^{\prime}$ and $\mathrm{G}^{\prime \prime}$ upon temperature increasing to $80^{\circ} \mathrm{C}$ supposedly due to the accelerated aggregation caused by the screened intermolecular repulsion by $\mathrm{NaCl}$. Upon further increase in $\mathrm{NaCl}$ concentration to $250 \mathrm{mM}$, the increase of $G^{\prime}$ and $G^{\prime \prime}$ was comparable to the samples with $100 \mathrm{mM} \mathrm{NaCl}$. Plotting the $\mathrm{G}^{\prime}$ and $\mathrm{G}^{\prime \prime}$ values as a function of $\mathrm{NaCl}$ concentration as shown in Figure 8a, one can observe that the increase of $\mathrm{G}^{\prime}$ and $\mathrm{G}^{\prime \prime}$ is mainly dominated by $\mathrm{NaCl}$ concentration, the concentration of $\mathrm{BC}$ microfibrils showed only a small effect on $\mathrm{G}^{\prime}$ and $\mathrm{G}^{\prime \prime}$ at low $\mathrm{NaCl}$ concentration. In addition, the tangent $\delta$ (Figure $8 \mathrm{~b}$ ) of the WPI-BC microfibril gels showed a comparable trend and value to that of pure WPI gels upon increasing $\mathrm{NaCl}$ concentration, suggesting that the WPI gel structure in the WPI-BC microfibril gel is structured in the same way as the pure WPI gel. ${ }^{46}$ Interestingly, tangent $\delta$ decreased upon addition of $\mathrm{NaCl}$ concentration, but remained constant up to $\mathrm{NaCl}$ concentration of $250 \mathrm{mM}$. The $\mathrm{G}^{\prime}$ and $\mathrm{G}^{\prime \prime}$ of all WPI-BC microfibril gels were independent of frequency as shown in Figure $6 c$ and $6 d$, The strain sweep in Figure 7 showed that the linear visco-elastic regime of the WPI gel with 0.4 wt\% BC microfibrils is the same as the pure WPI gel, at $\mathrm{NaCl}$ concentration of $250 \mathrm{mM}$. 

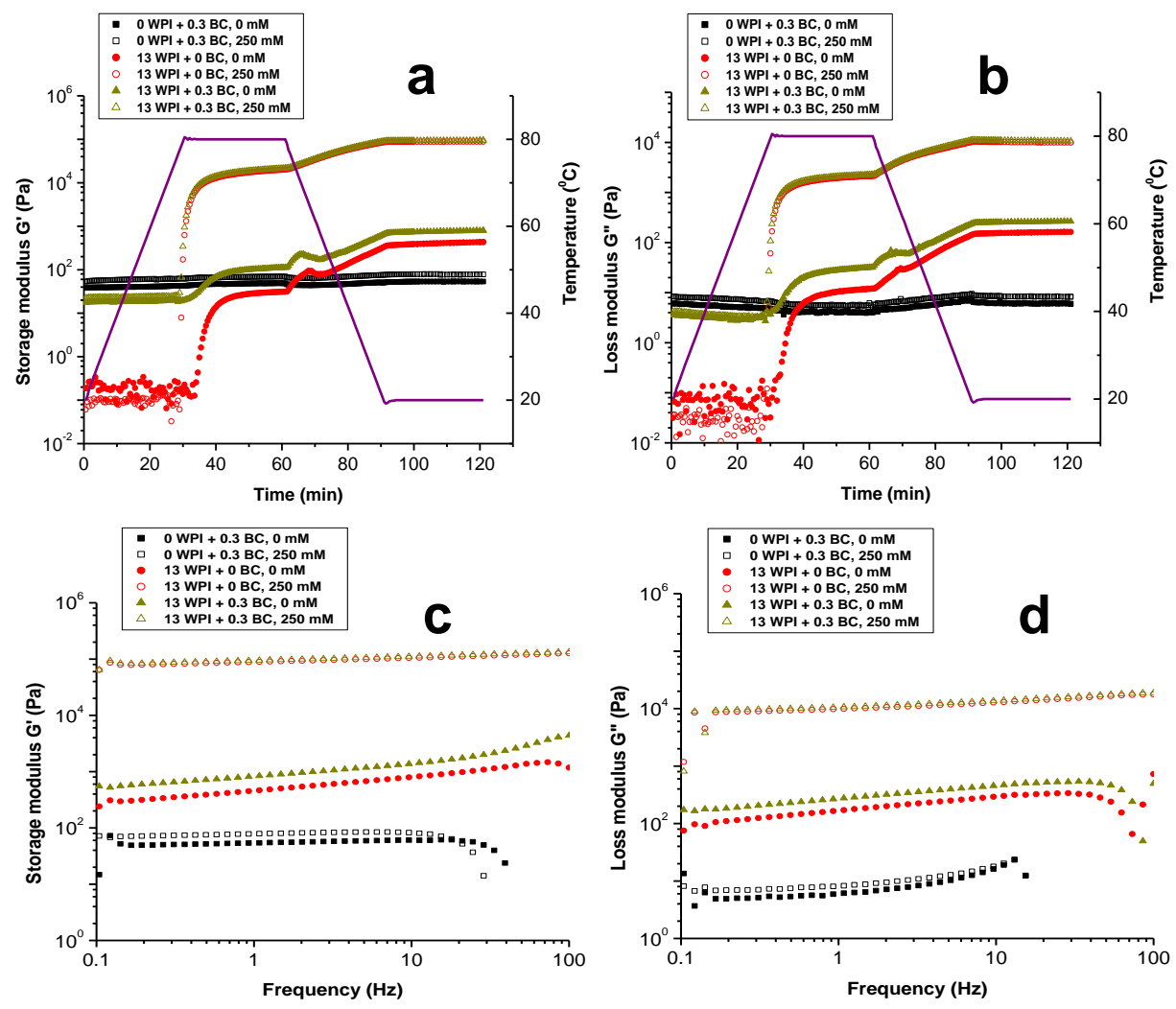

Figure 9 Comparison of gel rheological properties of 13 wt\% pure WPI, 0.3 wt\% pure BC microfibrils, and 13 wt\% WPI containing $0.3 \mathrm{wt} \% \mathrm{BC}$ microfibrils, at both 0 and $250 \mathrm{mM} \mathrm{NaCl}$. The development of storage modulus G' and loss modulus G" during gel formation of the samples as a function of time at a strain of $0.1 \%$ and frequency of $1 \mathrm{~Hz}$ is shown in figures (a) and (b). The purple line represents the temperature profile. Figures (c) and (d) show storage modulus $G^{\prime}$ (c) and loss modulus G" (d) of the gels as a function of frequency at a strain of $0.1 \%$ at $20^{\circ} \mathrm{C}$.

In Figure 9, the gel storage modulus $G^{\prime}$ and loss modulus $G^{\prime \prime}$ of pure WPI, pure BC microfibrils, and the WPI-BC microfibril mixtures at both 0 and $250 \mathrm{mM} \mathrm{NaCl}$ are presented. The $\mathrm{G}^{\prime}$ and $\mathrm{G}^{\prime \prime}$ of $0.3 \mathrm{wt} \%$ pure $\mathrm{BC}$ microfibril dispersion remained constant upon heating and cooling, both at 0 and $250 \mathrm{mM}$. This again suggests that $B C$ is stable against heating both at 0 and $250 \mathrm{mM} \mathrm{NaCl}$, an observation in line with the DSC data and literature. $^{22,23}$ The pure 13 wt\% WPI showed slower increase of G' and G" at $0 \mathrm{mM} \mathrm{NaCl}$ 
than that of the sample at $250 \mathrm{mM} \mathrm{NaCl}$, when the temperature reaches $80^{\circ} \mathrm{C}$. The $\mathrm{G}^{\prime}$ and $\mathrm{G}^{\prime \prime}$ of the gel were also much higher for $250 \mathrm{mM} \mathrm{NaCl}$ than for $0 \mathrm{mM} \mathrm{NaCl}$. In the mixture containing 13 wt\% WPI and 0.3 wt\% BC microfibrils, the development of G' and G' at both 0 and $250 \mathrm{mM}$ was similar to that of pure $13 \mathrm{wt} \%$ WPI gel, implying that the WPI gel is the dominant one and the addition of BC microfibrils has a negligible effect. However, the value of the $G^{\prime}$ and $G^{\prime \prime}$ in the mixture is higher than that of the pure WPI gel at $0 \mathrm{mM} \mathrm{NaCl}$, indicating a synergistic effect upon addition of $\mathrm{BC}$ microfibrils. At $250 \mathrm{mM} \mathrm{NaCl}$, the values of the $\mathrm{G}^{\prime}$ and $\mathrm{G}^{\prime \prime}$ of the mixture is close to that of the pure WPI gel, suggesting that WPI gel dominates the rheological properties of the mixed gel and the synergistic effect caused by $\mathrm{BC}$ microfibrils was too small in comparison to the strength of the WPI gel. In addition, the $G^{\prime}$ and $G^{\prime \prime}$ of $B C$ microfibrils were independent of frequency at both $0 \mathrm{mM}$ and 250 $\mathrm{mM} \mathrm{NaCl}$. The $\mathrm{G}^{\prime}$ and $\mathrm{G}^{\prime \prime}$ of the WPI-BC microfibril gels at both 0 and $250 \mathrm{mM} \mathrm{NaCl}$ showed similar dependency on frequency as the pure WPI gels at the same WPI concentration, indicating that WPI gel is the dominant one in the mixture.

With increase of the BC microfibril concentration, a self-supporting network was formed. The minimal gel concentration was found to be $0.01 \mathrm{wt} \%$ at $1 \mathrm{~cm}$ sample height. ${ }^{32}$ The formation of a gel by BC microfibrils was also confirmed by rheology. ${ }^{32}$ However, BC microfibrils are non-gelling agents upon heating. Therefore, WPI is the only gelling agent during heating. Our results showed that the presence of $B C$ microfibrils did not influence the WPI gelation upon heating. However, adding high enough BC microfibril concentration leads to a firmer WPI gel (without added salt). This increase is not observed when $\mathrm{NaCl}$ is added. Instead, in that case the WPI-BC microfibril gels show the same rheological properties as the pure WPI gels. This is probably related to the fact that the increase of gel firmness resulted from $\mathrm{NaCl}$ is much larger than the increase of gel firmness resulted from BC microfibrils addition up to 0.4 wt\%. Consequently, the change of $G^{\prime}$ and $G^{\prime \prime}$ of the gels are dominated by $\mathrm{NaCl}$ concentration, resulting in stronger WPI gels. Adding $\mathrm{NaCl}$ alters the gel structure from fine-stranded to particulate gels and the firmness of the WPI gel increases, with bigger aggregate present. Our results on firmer gels upon salt increase are in agreement with the literature. ${ }^{12}$

In conclusion, we found that high enough BC micrfibril concentration leads to increase in the firmness (synergistic effect) of the WPI gels. The reasons can be two-fold. On one hand, the amount of the solvent entrapped in the BC phase could be increased, thus, leading to 
the concentration of the protein phase, and increase of the gel firmness. ${ }^{47}$ On the other hand the $\mathrm{BC}$ network itself could lead to an increase in firmness by itself, and this could then add to the overall gel strength.

\subsubsection{Confocal laser scanning microscopy (CLSM)}

To further investigate the WPI-BC microfibril gels at different $\mathrm{BC}$ microfibrils and $\mathrm{NaCl}$ concentrations, we studied the microstructure of the gels using CLSM. 


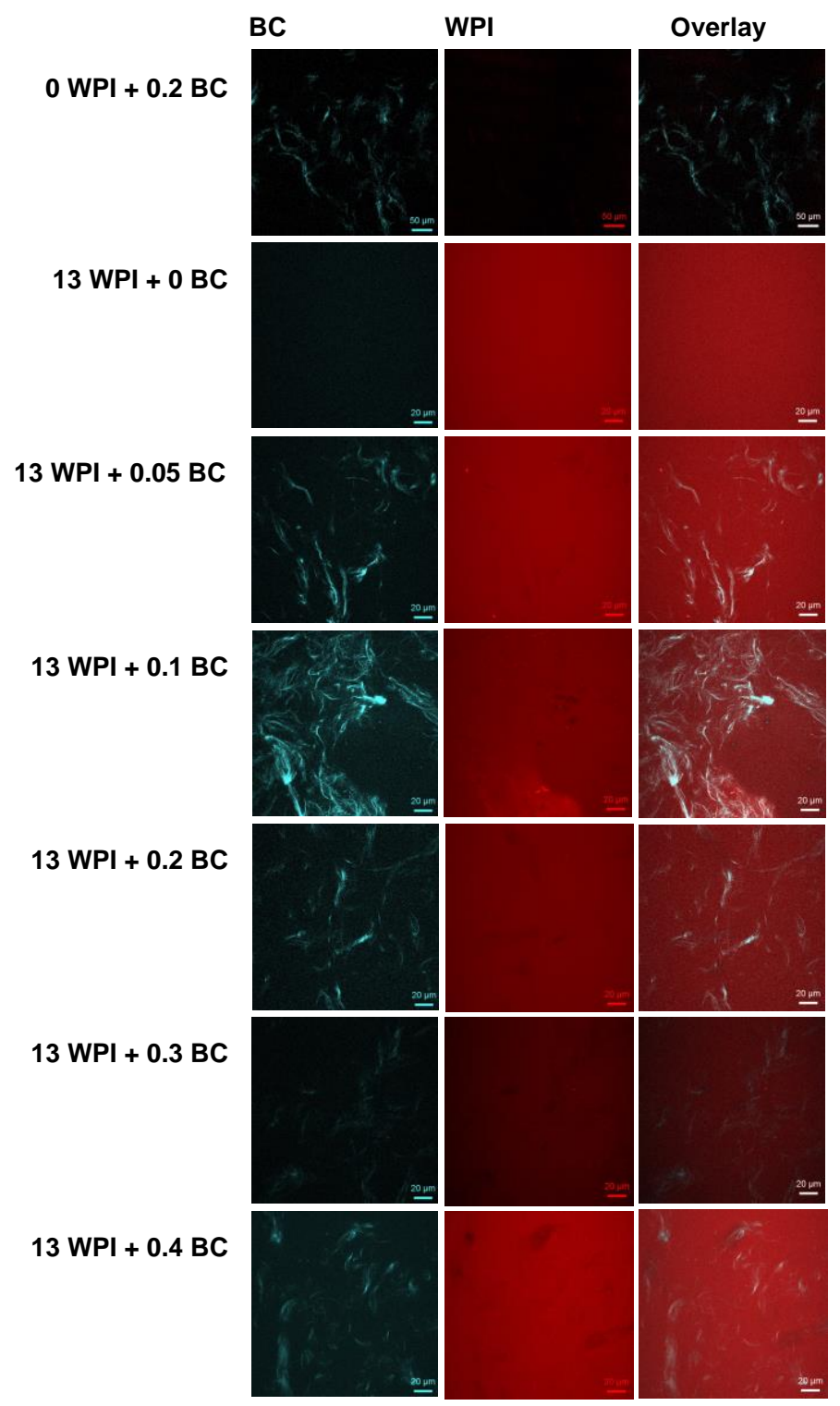

Figure 10 CLSM images of WPI-BC microfibril gels containing 13 wt\% WPI and BC microfibril concentrations of $0,0.05,0.1,0.2,0.3$ and $0.4 \mathrm{wt} \%$. The concentrations (wt\%) are indicated next to the images. For each sample, images from the BC microfibril channel, WPI channel and the overlay image were shown. WPI is shown in red and BC microfibril in turquoise. The scale bar corresponds to $20 \mu \mathrm{m}$. 


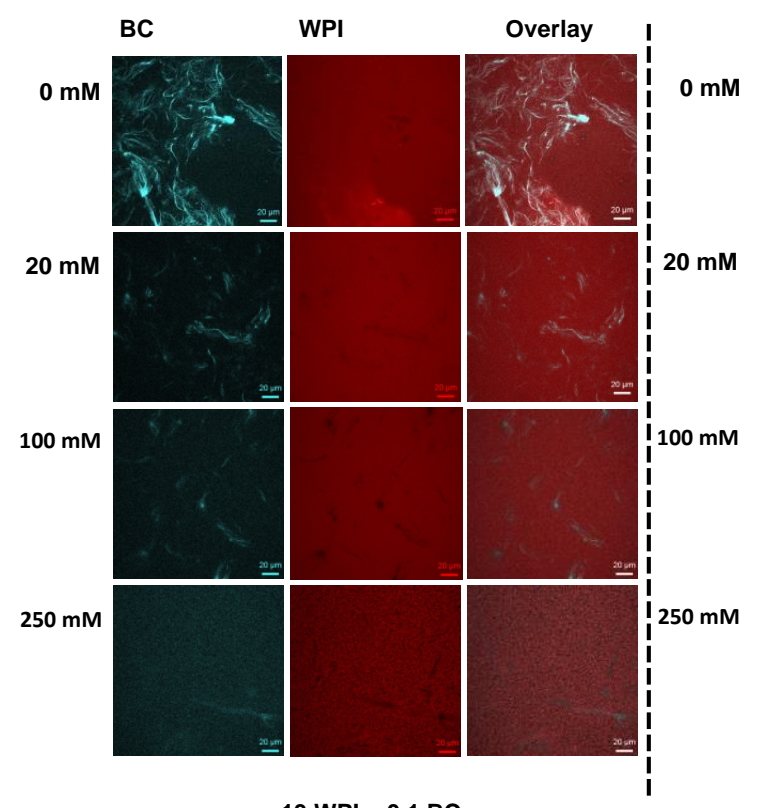

$13 \mathrm{WPI}+0.1 \mathrm{BC}$
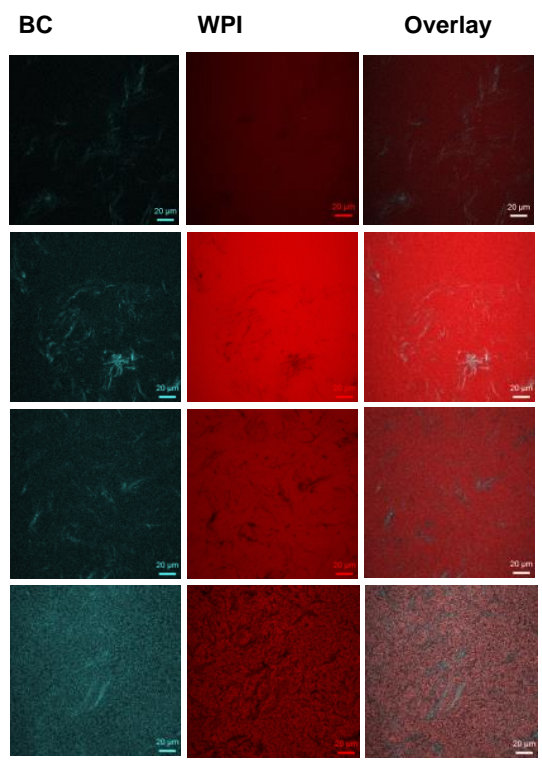

$13 \mathrm{WPI}+0.3 \mathrm{BC}$

Figure 11 CLSM images of WPI-BC microfibril gels with $\mathrm{NaCl}$ of $0,20,100$ and $250 \mathrm{mM}$. The $\mathrm{NaCl}$ concentrations $(\mathrm{mM})$ are indicated on the left side of the images. The WPI and BC concentrations (wt\%) are indicated below the images. Images from the BC microfibril channel, WPI channel and the overlay image were shown. The BC microfibrils are shown in turquoise and the WPI is shown in red. The scale bar corresponds to $20 \mu \mathrm{m}$. The black dashed line in the middle of the figure separates the samples containing 13 wt\% WPI and 0.1 wt\% BC microfibrils from the samples containing 13 wt\% WPI and 0.3 wt\% BC microfibrils. 


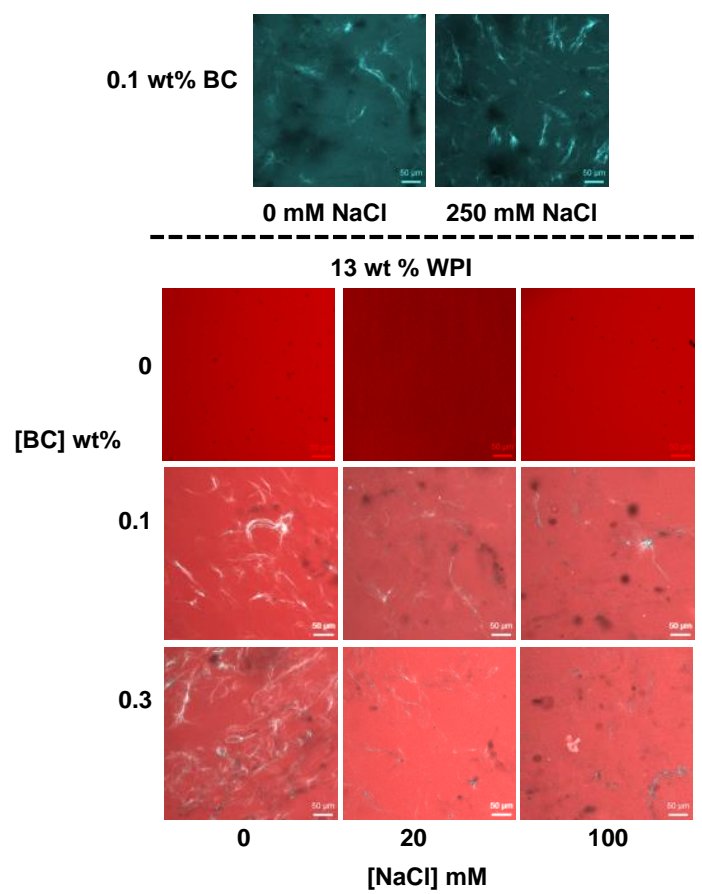

Figure 12 CLSM overlay images of 13 wt\% WPI gels mixed with $0,0.1$ and $0.3 w t \%$ BC microfibrils. The $\mathrm{NaCl}$ concentrations in the samples $(0,20$ and $100 \mathrm{mM})$ are indicated below the images. The $\mathrm{BC}$ concentration (wt\%) are indicated on the left side of the images. The scale bar corresponds to $50 \mu \mathrm{m}$. The images shown above the black dashed line are from $0.1 \mathrm{wt} \%$ pure BC microfibril at concentrations of 0 and $250 \mathrm{mM} \mathrm{NaCl}$.

In Figure 10, we observed a homogeneous WPI network in pure 13 wt\% WPI gel. In WPI gels containing BC microfibrils, we also observed a homogeneous network for the WPI, independent of the concentration of BC microfibrils. In addition, we observed clusters of $B C$ microfibrils distributed over the system. No macroscopic phase separation could be observed for the any of the BC microfibril concentrations. The distribution of these clusters of $B C$ microfibrils and corresponding voids (ranging from tens to thousands of micrometers) varies depending on concentration of $B C$ microfibrils, which has been reported previously. ${ }^{32}$ In the CLSM overlay images where both WPI and BC microfibril are shown, we observed that WPI forms a homogeneous gel network (on the length scale probed by CLSM), even when the BC microfibrils are present. This suggests that the presence of $B C$ microfibrils does not influence the WPI gel network structure on the length 
scale of micrometer or larger. Similar results have also been reported in WPI gels with other protein $^{48}$ or polysaccharide ${ }^{10}$ systems.

When adding $\mathrm{NaCl}$, we observed that the WPI-BC duplex gels become more opaque. We observed (Figure 11) that increasing the concentration of $\mathrm{NaCl}$ left the distribution of $\mathrm{BC}$ microfibrils in the gels unaffected, while the WPI gels become coarser. The coarseness increased with increasing $\mathrm{NaCl}$ concentration. The amount of $\mathrm{BC}$ microfibrils $(0.1 \mathrm{wt} \%$ or $0.3 \mathrm{wt} \%)$ did not influence the structure of the duplex gels significantly. Previous studies have shown that adding $\mathrm{NaCl}$ has a significant influence on the gelation of WPI, where the gel network becomes coarser by changing from a fine-stranded to a particulate gel. ${ }^{49,50} \mathrm{BC}$ microfibril gel structure was found to be unaffected by $\mathrm{NaCl}$ concentration, as observed in the CLSM images (Figure 12). Adding different concentrations of $\mathrm{NaCl}$ to the WPI-BC microfibril duplex gels showed that only the structure of the WPI gels was influenced, to an extent that is similar to that of the pure WPI gels.

\subsubsection{Scanning electron microscopy (SEM)}

SEM was used to determine the morphology of the WPI-BC microfibril duplex gels at different $\mathrm{BC}$ microfibril and $\mathrm{NaCl}$ concentrations. 


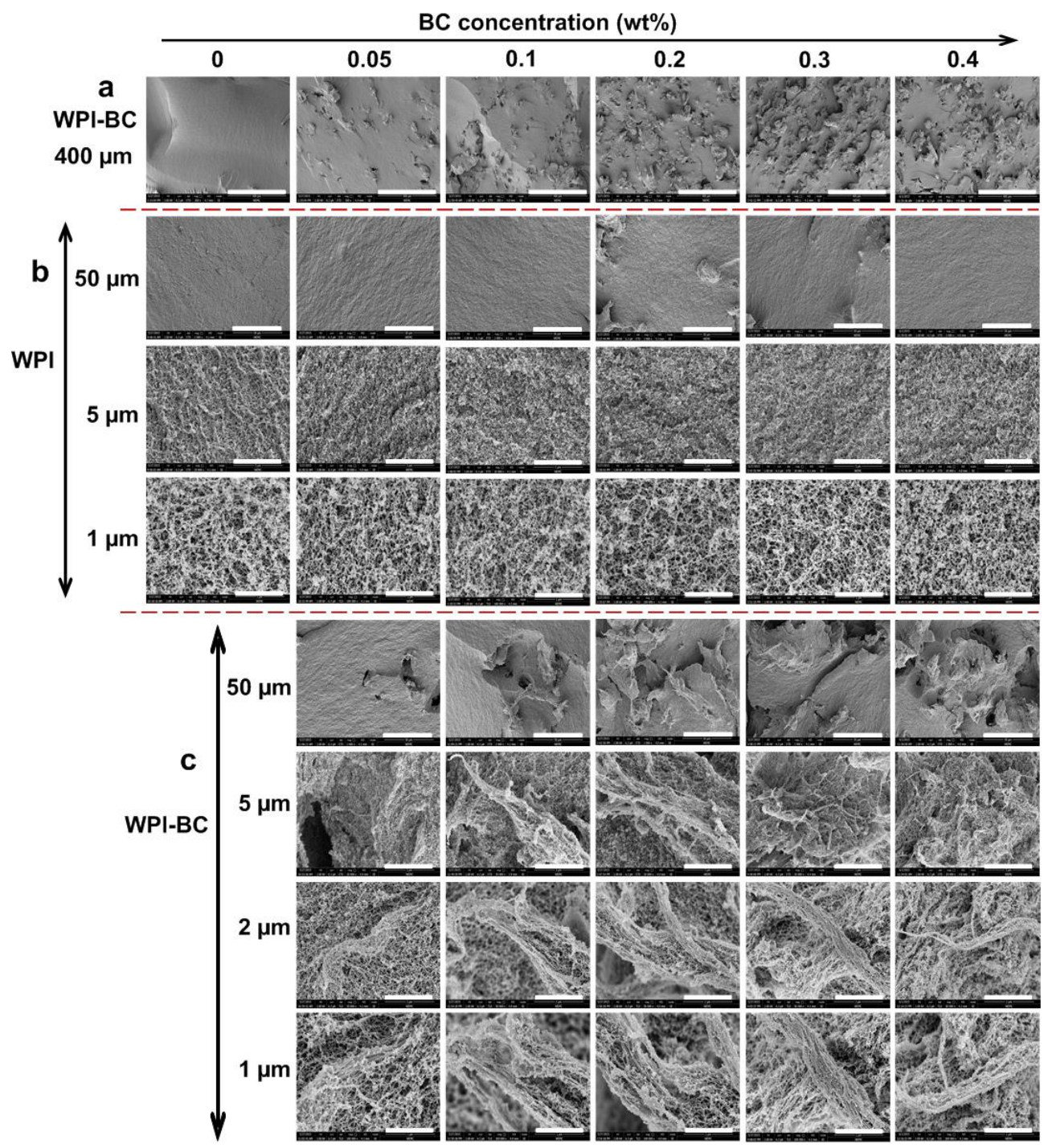

Figure 13 SEM images of 13 wt\% WPI containing $0,0.05,0.1,0.2,0.3$ and 0.4 wt\% BC microfibrils. The overall structure of the gels at low magnification is shown in (a). An area where mainly WPI is present is shown in (b). An area where both WPI and BC microfibrils are present is shown in (c). Scale bars correspond to $400 \mu \mathrm{m}, 50 \mu \mathrm{m}, 5 \mu \mathrm{m}, 2 \mu \mathrm{m}$ and $1 \mu \mathrm{m}$.

The pure WPI gel showed a smooth and homogeneous structure, as shown in Figure 13a. Upon addition of BC microfibrils, we observed a homogeneous WPI gel with BC 
microfibrils distributing uniformly in the WPI gel. Increasing the BC microfibril concentration from $0.05 \mathrm{wt} \%$ to $0.4 \mathrm{wt} \%$ in WPI gels, the heterogeneity of the gel also increased. $^{32}$ To have a closer look at the morphology of the WPI gel with different concentrations of BC microfibrils, we imaged the sample area where mainly WPI is present, as shown in Figure $13 \mathrm{~b}$. The WPI gel structure formed in samples containing BC microfibrils were similar to the structure of a pure WPI gel, independent of BC microfibril concentration, suggesting that the presence of $B C$ microfibrils did not significantly influence the WPI gel structure. When the area where both WPI and BC microfibrils are present was imaged, we observed BC microfibril bundles and the dimensions of the bundles vary. In addition, we also observed the growth of WPI protein structures along the BC microfibril bundles, presumably either covering the surface of the BC microfibril bundles or being close to the $\mathrm{BC}$ microfibril bundles. It is not clear whether this is due to specific adsorption of WPI on the BC microfibril surface or simply due to the fact that the WPI proteins and according structures are close to the BC bundles. To check whether WPI actually adsorbed on BC microfibril surface, we centrifuged the WPI-BC microfibril dispersions and determined the WPI concentration in the supernatant (results not shown). We found that the value was comparable to that of the initial WPI concentration in the dispersion, suggesting no specific adsorption of WPI on BC, although we cannot exclude limited adsorption of WPI on the surface of the BC microfibrils by this one type of experiment. 


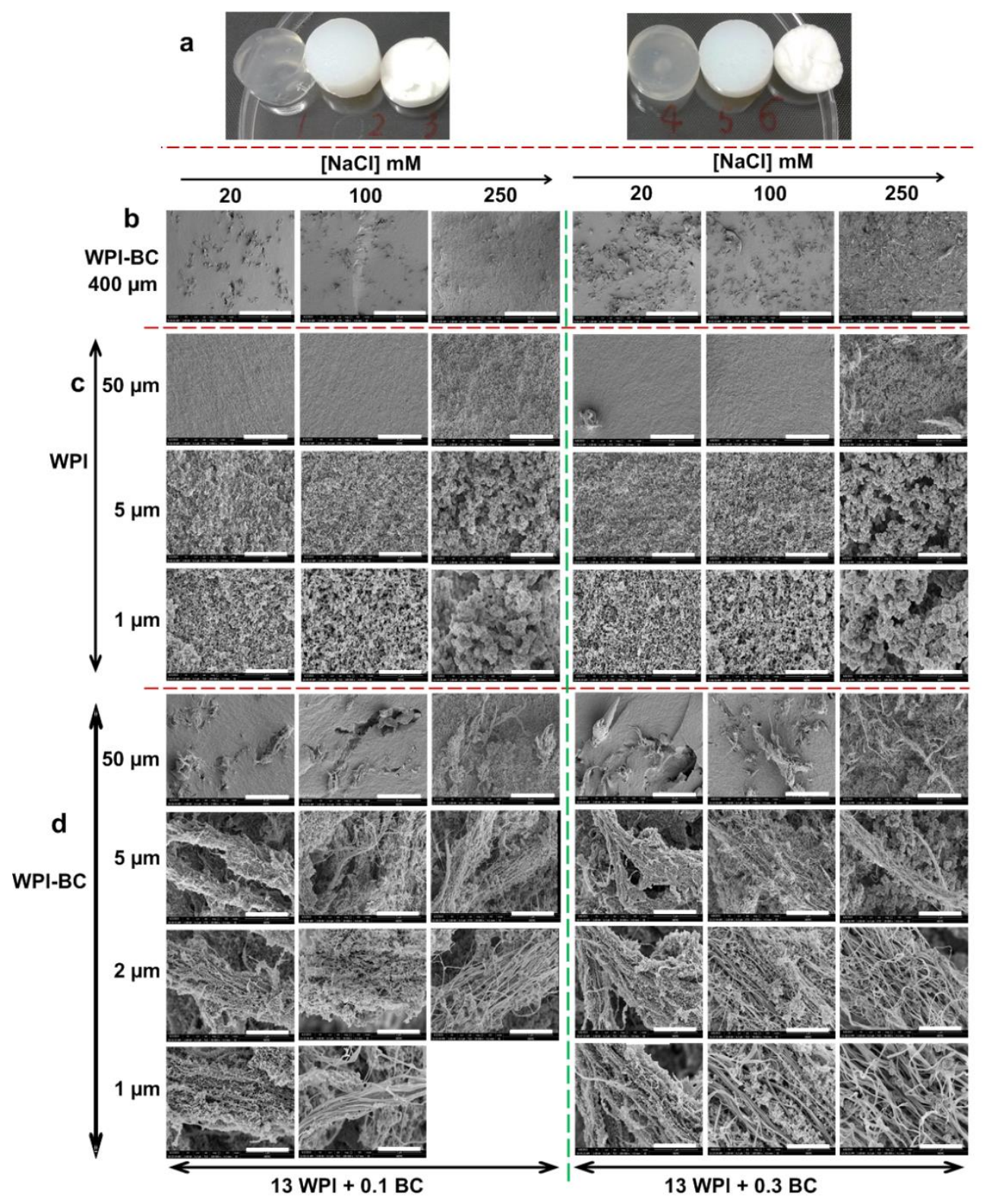

Figure 14 SEM images of 13 wt\% WPI containing $0,0.1$, and $0.3 w t \% \mathrm{BC}$ microfibrils at $\mathrm{NaCl}$ concentraiton of 20,100 , and $250 \mathrm{mM}$. The visual appearance of the gels are shown in (a).The overall structure of the gels at low magnification is shown in (b). An area where mainly WPI is present is shown in (c). An area where both WPI and BC microfibrils are present is shown in (d). 
The $\mathrm{NaCl}$ concentration (mM) is indicated on the top of the images. Scale bars correspond to $400 \mu \mathrm{m}, 50 \mu \mathrm{m}, 5 \mu \mathrm{m}, 2 \mu \mathrm{m}$ and $1 \mu \mathrm{m}$. The green dashed line in the middle of the figure separates the samples containing 13 wt\% WPI and 0.1 wt\% BC microfibrils from the samples containing 13 wt\% WPI and 0.3 wt\% BC microfibrils.

When $20 \mathrm{mM} \mathrm{NaCl}$ is added, the WPI-BC microfibril duplex gels become translucent. Upon increasing $\mathrm{NaCl}$ concentration to $250 \mathrm{mM}$, the gels become increasingly opaque (Figure 14a). An increase in coarseness of the WPI gel is also observed, as shown in Figure 14b and 14c. Adding 0.1 or 0.3 wt\% BC microfibrils did not show a significant difference on the macroscopic appearance of the gels. At higher magnification, we observed a gel structure changing from fine-stranded to particulate gel with increasing $\mathrm{NaCl}$ concentration (Figure $14 \mathrm{~b}$ and 14c). This is related to reduced electrostatic repulsion between proteins at higher $\mathrm{NaCl}$ concentration, facilitating the protein aggregation into a particulate gel structure. ${ }^{51}$ The influence of $\mathrm{BC}$ microfibrils on the WPI gel structure with added $\mathrm{NaCl}$ was comparable to the gels without added $\mathrm{NaCl}$. The $\mathrm{BC}$ microfibril bundles uniformly distributed in the WPI gel and did not influence the WPI gel structure. Interestingly, with increase in $\mathrm{NaCl}$ concentration, we observe more BC microfibril surface that seems to be not covered or hidden by protein (Figure 14d). This may be due to the stronger aggregation of the proteins among themselves at larger $\mathrm{NaCl}$ concentration. The presence of $\mathrm{BC}$ microfibrils did not show a distinct influence on the structure of the duplex gel. It can be concluded that the addition of $\mathrm{NaCl}$ in WPI-BC microfibril duplex gels only influenced the protein aggregation, leading to a coarser protein gel, to a similar extent as to what is observed in pure WPI gels upon increasing $\mathrm{NaCl}$ concentration. This is in-line with the DSC results, rheological properties, and CLSM images, where also no specific interactions between WPI and $\mathrm{BC}$ microfibrils could be concluded.

From the CLSM and SEM images, we can conclude that adding BC microfibrils did not influence the WPI gel structure. Overall, for any $\mathrm{NaCl}$ concentration, in duplex gels of WPI and $B C$ microfibrils, the WPI forms a gel structure independent from the BC microfibril gel, and with a structure similar to that in a pure WPI gel. 


\subsubsection{Large deformation response}

The breakdown of gels under large deformation was also studied. ${ }^{52}$ To investigate how BC microfibrils affect the breakdown properties of the WPI gels at different $\mathrm{NaCl}$ concentrations, large deformation rheology was performed. The macroscopic appearance of the gels is shown in Figure 15 and the true stress and true strain of gels under uniaxial deformation are shown in Figure 16. Figure 17 shows the Young's modulus and fracture stress as a function of BC microfibril concentration.

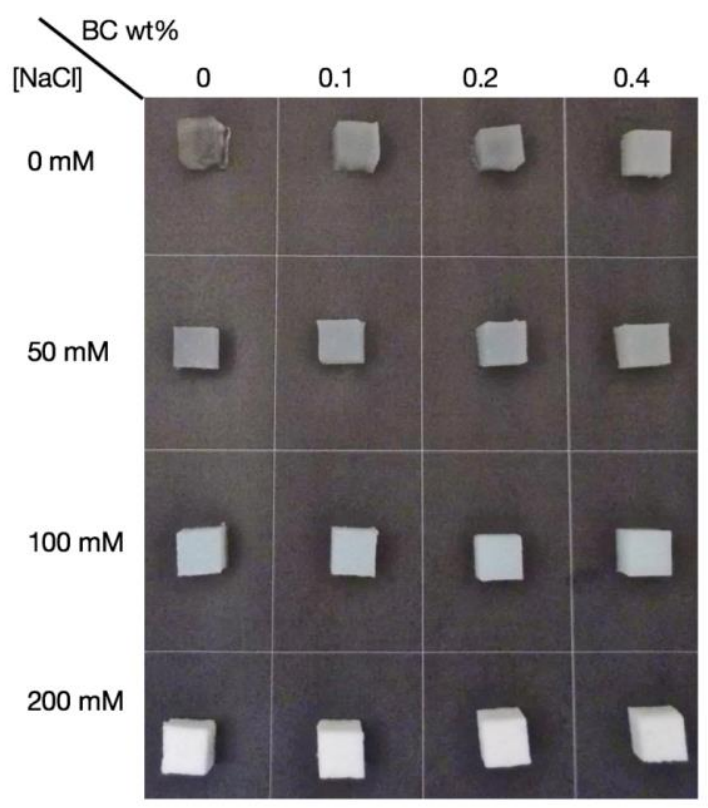

Figure 15 Visual observation of WPI-BC microfibril duplex gels containing 13 wt\% WPI and BC microfibril concentrations of $0,0.1,0.2$, and $0.4 \mathrm{wt} \%$. The corresponding $\mathrm{NaCl}$ concentration in the gels is indicated on the left side of the images. 

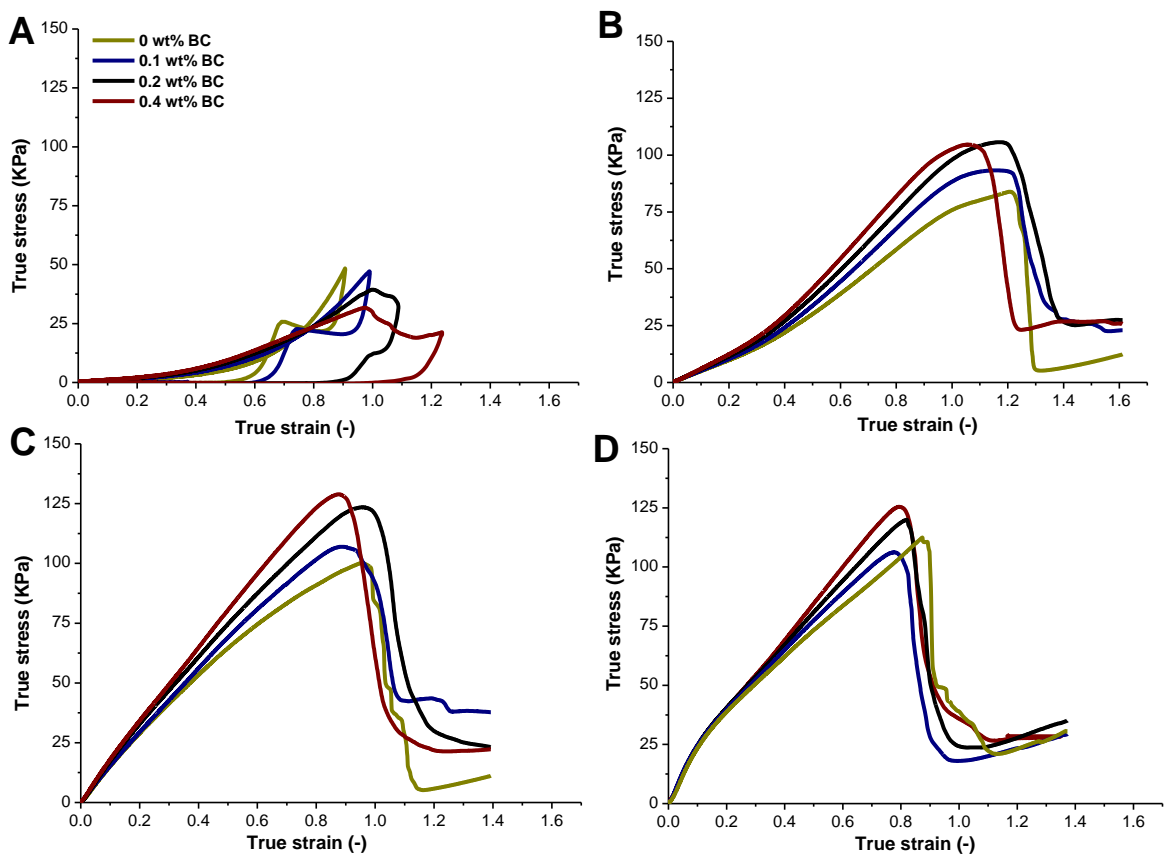

Figure 16 The True stress (KPa) over true strain (-) of WPI-BC microfibril gels containing 13 wt\% WPI and BC microfibrils of $0,0.1,0.2$ and 0.4 wt\%. The $\mathrm{NaCl}$ concentration in $\mathrm{A}, \mathrm{B}, \mathrm{C}, \mathrm{D}$ corresponds to $0,50,100$ and $200 \mathrm{mM}$. 

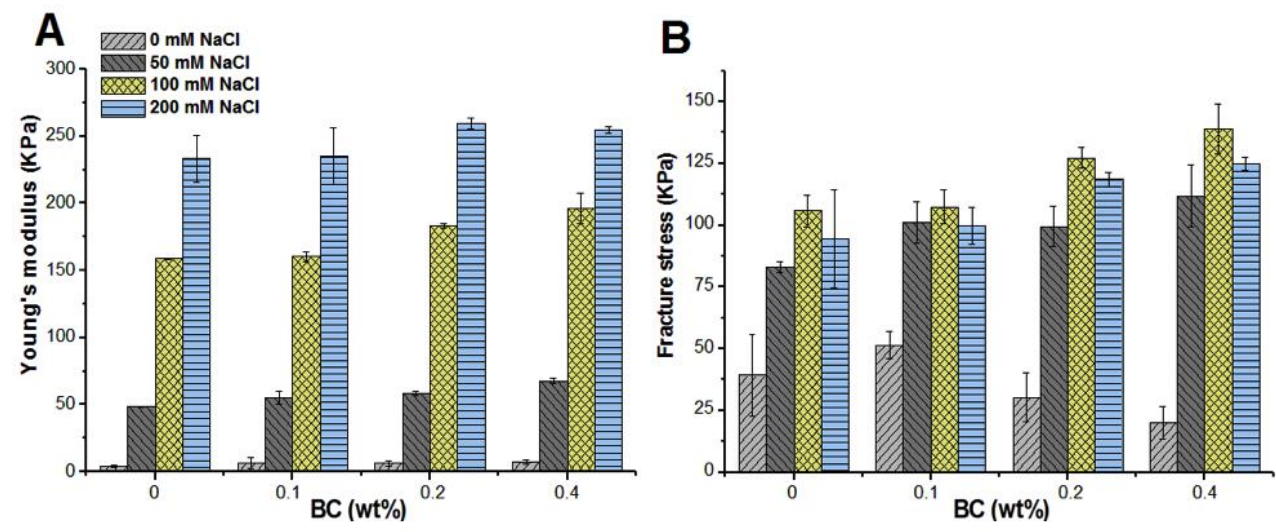

Figure 17 Young's modulus (A) and fracture stress (B) of WPI-BC microfibril gels as a function of BC microfibril concentration. The concentration of WPI is $13 \mathrm{wt} \%$ in all samples.

Both increasing $\mathrm{BC}$ microfibril and $\mathrm{NaCl}$ concentrations resulted in more opaque gels, as shown above (Figure 15). For pure WPI gel without $\mathrm{NaCl}$ (Figure 16A), the gel was very weak and did not fracture completely under compression. With addition of BC microfibrils, the gels became stiffer, but were still weak and fractured only partially (Figure 16A). This is due to the high elastic properties of WPI gel. Adding BC microfibrils decrease the elasticity of the duplex gels, in line with the small deformation rheological properties, but the gels were still not brittle enough to fracture completely. With addition of $50 \mathrm{mM} \mathrm{NaCl}$, all the gels eventually fractured. Upon increase in $\mathrm{NaCl}$ concentration, the gels fractured at larger stress but lower strain, corresponding to a firmer but more brittle gel with a coarser gel structure. ${ }^{12,53}$ We note that the fracture properties of WPI-BC microfibril gels with 100 $\mathrm{mM} \mathrm{NaCl}$ are comparable to those of gels with $200 \mathrm{mM} \mathrm{NaCl}$. This is related to the balance between formation of larger aggregates and less connectivity between the aggregates. ${ }^{54,55}$ Overall, the fracture behaviour of the WPI gels with BC microfibrils under large deformation is comparable to that of the pure WPI gels, independent of BC microfibril concentration. The difference in fracture behaviour induced by the $\mathrm{NaCl}$ addition was attributed to WPI gel structural changes, as confirmed by CLSM and SEM images. In Figure $17 \mathrm{~B}$, it also shows that the concentration of $\mathrm{BC}$ microfibrils in the WPI-BC mirofibril duplexgel did not significantly alter the fracture stress of the gels in comparison to the pure WPI gels at the same concentration. This is due to the strong gel formed by WPI, 
which dominates the gel facture properties. Our results are in agreement with literature in that for mixed gels with two independent networks, the stronger gel of the two dominates the fracture behaviour. ${ }^{48}$

Young's modulus was determined from the initial slope of the stress over strain curve (strain 0.01-0.1). As shown in Figure 17A, the Young's modulus of the pure WPI gels increased significantly with increasing $\mathrm{NaCl}$ concentration, due to the formation of thicker protein strands. Upon BC microfibrils addition, the Young's modulus slightly increased with increasing $\mathrm{BC}$ microfibril concentration in the duplex gels, with the exception of the gels prepared at $200 \mathrm{mM} \mathrm{NaCl}$ where the Young's modulus is comparable to that of the pure WPI gels with $200 \mathrm{mM} \mathrm{NaCl}$. This implies that the Young's modulus of the duplex gels is dominated by the WPI gel.

\subsubsection{Water holding capacity (WHC)}

Water holding capacity of the WPI-BC microfibril gels was determined at varying concentrations of $\mathrm{BC}$ microfibrils and $\mathrm{NaCl}$.

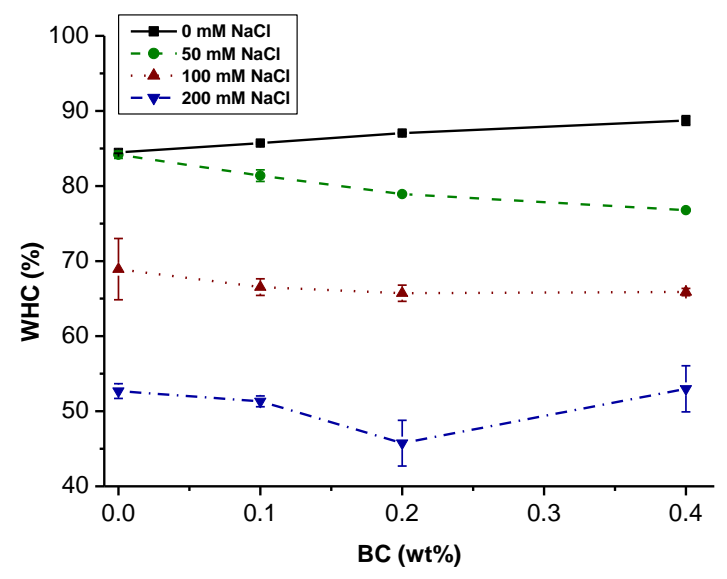

Figure 18 WHC of WPI-BC microfibril duplex gels containing 13 wt\% WPI and BC concentrations of $0,0.1,0.2$ and $0.4 \mathrm{wt} \%$. The $\mathrm{NaCl}$ concnetrations in the duplex gels vary from 0,50 , and 100 to 200 $\mathrm{mM}$. 
In WPI-BC microfibril duplex gels without $\mathrm{NaCl}$, the WHC increased slightly with increasing $\mathrm{BC}$ microfibril concentration. This is attributed to the good water binding ability of $\mathrm{BC}{ }^{20}$ which has also been reported in other studies. ${ }^{22,56,57}$ Upon addition of $\mathrm{NaCl}$, the WHC of the gels decreased with increase in $\mathrm{NaCl}$ concentration. At a $\mathrm{NaCl}$ concentration of $50 \mathrm{mM}$, the WHC gradually decreased with increasing $B C$ concentration. At $100 \mathrm{mM} \mathrm{NaCl}$, the decrease of the WHC is less pronounced with increase of $B C$ microfibril concentration. When $200 \mathrm{mM} \mathrm{NaCl}$ is added, the WHC of the WPI-BC microfibril duplex gels is comparable to that of the pure WPI gels. In line with literature, the WHC of pure WPI gels decreases with increasing $\mathrm{NaCl}$ concentration. ${ }^{12,53,55}$ The decreased WHC of WPI gels due to addition of $\mathrm{NaCl}$ is related to a more coarse microstructure. Meanwhile, addition of $\mathrm{NaCl}$ was also found to decrease the WHC of BC mcirofibrils. ${ }^{39}$ Expectedly, duplex gels of WPI and BC microfibrils exhibit a decrease of WHC upon addition of $\mathrm{NaCl}$ at 100 and 200 $\mathrm{mM} \mathrm{NaCl}$, the WHC is less influenced by the BC microfibrils; in fact the WHC of the duplex gels is comparable to that of the pure WPI. . This is presumably due to the fact that the WHC of WPI is already rather low at higher salt concentrations due to the coarser structure. Previous results showed that the microstructure and stiffness of gels show a correlation to their WHC. ${ }^{38,55}$ We accordingly plotted the Young's modulus as a function of WHC in Figure 19.

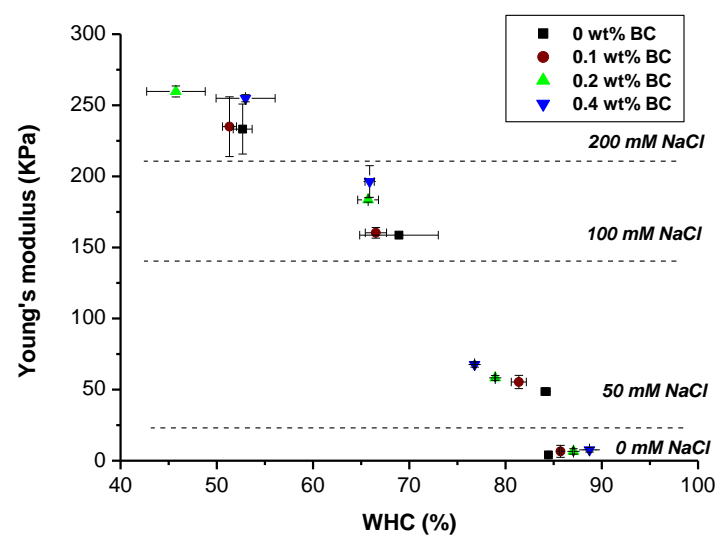

Figure 19 Young's modulus as a function of WHC of WPI-BC microfibril duplex gels containing 13 wt\% WPI and BC concentrations of $0,0.1,0.2$ and 0.4 wt\% at $\mathrm{NaCl}$ concentrations of $0,50,100$, $200 \mathrm{mM}$. Dashed lines are used to separate the regions at different $\mathrm{NaCl}$ concentrations. 
For pure WPI gels, the Young's modulus showed a negative correlation with the WHC. With increasing $\mathrm{NaCl}$ concentration, the stiffness and coarseness of the gel increased, thereby facilitating the water removal. This result is in line with earlier work on WPI gels by Urbonaite et al. ${ }^{55}$ The addition of different concentrations of BC microfibrils to the WPI did not change the relation between the Young's modulus and WHC significantly. This again confirmed that the WHC of the WPI-BC microfibril duplex gels is dominated by the properties of the WPI gel, i.e. the concentration of $\mathrm{NaCl}$ in this study.

\subsection{Conclusions}

We have shown that the WPI gel structure within a WPI-BC microfibril gel is independent of the presence of BC microfibrils. The gel consists of a WPI gel network and a BC microfibrillar network. We therefore call it a duplex gel. In the duplex gels, $\mathrm{NaCl}$ only influences the WPI gel structure. At lower $\mathrm{NaCl}$ concentrations, the rheological properties of WPI gels can be altered by the addition of $\mathrm{BC}$ microfibrils. At higher $\mathrm{NaCl}$ concentration, the rheological properties are dominated by the (much stronger) WPI gel. The presence of $B C$ microfibrils did not show a significant influence on the WPI gelation dynamics and gel structure. The concentration of $\mathrm{BC}$ microfibrils did not significantly influence the large deformation rheology nor the WHC of the WPI-BC microfibril duplex gels. Properties of the gels are dominated by the properties of the WPI gel. The ability of the BC microfibril gel to modify the WPI gel properties in the duplex gel is the largest for the weakest WPI gel structure and increases with increasing concentration of $B C$ microfibrils.

\subsection{Acknowledgement}

This work is supported by NanoNextNL, a micro and nanotechnology consortium of the Government of the Netherlands and 130 partners. We thank Norbert de Ruijter for CLSM image analysis and Tiny Franssen-Verheijen for SEM image analysis. We thank Dr. A. Kijk and Dr. S. Veen for providing the dispersion of bacterial cellulose. 


\subsection{Reference}

1. V. Tolstoguzov, Food Hydrocolloids, 1991, 4, 429-468.

2. J.-L. Doublier, C. Garnier, D. Renard and C. Sanchez, Current Opinion in Colloid \& Interface Science, 2000, 5, 202-214.

3. V. Y. Grinberg and V. Tolstoguzov, Food Hydrocolloids, 1997, 11, 145-158.

4. C. G. De Kruif, F. Weinbreck and R. de Vries, Current Opinion in Colloid \& Interface Science, 2004, 9, 340-349.

5. M. Corredig, N. Sharafbafi and E. Kristo, Food Hydrocolloids, 2011, 25, 1833-1841.

6. E. Dickinson, Trends in Food Science \& Technology, 1998, 9, 347-354.

7. S. Turgeon, M. Beaulieu, C. Schmitt and C. Sanchez, Current Opinion in Colloid \& Interface Science, 2003, 8, 401-414.

8. J. De Wit, Journal of Dairy Science, 1998, 81, 597-608.

9. J. M. Aguilera, Food technology, 1995, 49, 83-89.

10. S. L. Turgeon and M. Beaulieu, Food Hydrocolloids, 2001, 15, 583-591.

11. D. Zasypkin, E. Dumay and J. Cheftel, Food Hydrocolloids, 1996, 10, 203-211.

12. S. Barbut, Food Research International, 1995, 28, 437-443.

13. C. M. Bryant and D. J. McClements, Food Hydrocolloids, 2000, 14, 383-390.

14. S. Mleko, E. C. Y. Li-Chan and S. Pikus, Food Research International, 1997, 30, 427-433.

15. C. Capitani, O. E. Pérez, B. Pacheco, M. Teresa and A. M. R. Pilosof, Food Hydrocolloids, 2007, 21, 1344-1354.

16. C. Tavares and J. L. Da Silva, International dairy journal, 2003, 13, 699-706.

17. J. T. Tobin, S. M. Fitzsimons, V. Chaurin, A. L. Kelly and M. A. Fenelon, Food Hydrocolloids, 2012, 27, 201-207.

18. A. R. Abhyankar, D. M. Mulvihill, M. A. Fenelon and M. A. E. Auty, Food Hydrocolloids, 2010, 24, 18-26.

19. R. J. Moon, A. Martini, J. Nairn, J. Simonsen and J. Youngblood, Chemical Society Reviews, 2011, 40, 3941-3994.

20. Z. Shi, Y. Zhang, G. O. Phillips and G. Yang, Food Hydrocolloids, 2014, 35, 539-545.

21. A. Okiyama, M. Motoki and S. Yamanaka, Food Hydrocolloids, 1992, 6, 479-487.

22. A. Okiyama, M. Motoki and S. Yamanaka, Food Hydrocolloids, 1993, 6, 503-511.

23. H. Ougiya, K. Watanabe, Y. Morinaga and F. Yoshinaga, Bioscience, Biotechnology, and Biochemistry, 1997, 61, 1541-1545.

24. H. Zhu, S. Jia, H. Yang, W. Tang, Y. Jia and Z. Tan, Food Science and Biotechnology, 2010, 19, 1479-1484.

25. P. Paximada, E. Tsouko, N. Kopsahelis, A. A. Koutinas and I. Mandala, Food Hydrocolloids, 2016, 53, 225-232. 
26. P. Paximada, A. A. Koutinas, E. Scholten and I. G. Mandala, Food Hydrocolloids, 2016, 54, 245254.

27. M. Phisalaphong and N. Chiaoprakobkij, Bacterial Cellulose: A Sophisticated Multifunctional Material, 2012, 9, 143-156.

28. S. Cho and N. Almeida, Dietary fiber and health, CRC Press, 2012.

29. K. Lin and H. Lin, Journal of Food Science, 2004, 69, SNQ107-SNQ111.

30. S. B. LIN, L. C. CHEN and H. H. CHEN, Journal of Food Process Engineering, 2011, 34, 1363-1379.

31. R. Jonas and L. F. Farah, Polymer Degradation and Stability, 1998, 59, 101-106.

32. A. Kuijk, R. Koppert, P. Versluis, G. van Dalen, C. Remijn, J. Hazekamp, J. Nijsse and K. P. Velikov, Langmuir, 2013, 29, 14356-14360.

33. S. J. Veen, A. Kuijk, P. Versluis, H. Husken and K. P. Velikov, Langmuir, 2014, 30, 13362-13368.

34. S. J. Veen, P. Versluis, A. Kuijk and K. P. Velikov, Soft Matter, 2015, 11, 8907-8912.

35. Y. Huang, C. Zhu, J. Yang, Y. Nie, C. Chen and D. Sun, Cellulose, 2014, 21, 1-30.

36. A. M. A. Gallegos, S. H. Carrera, R. Parra, T. Keshavarz and H. M. Iqbal, BioResources, 2016, 11, 5641-5655.

37. Z. Shi, Y. Zhang, G. O. Phillips and G. Yang, Food Hydrocolloids, 2014, 35, 539-545.

38. V. Urbonaite, H. H. J. de Jongh, E. van der Linden and L. Pouvreau, Journal of Agricultural and Food Chemistry, 2014, 62, 7550-7558.

39. Y. Chung and Y. Shyu, International Journal of Food Science \& Technology, 1999, 34, 23-26.

40. S. M. Fitzsimons, D. M. Mulvihill and E. R. Morris, Food Hydrocolloids, 2007, 21, 638-644.

41. J. I. Boye, I. Alli, A. A. Ismail, B. F. Gibbs and Y. Konishi, International dairy journal, 1995, 5, 337353.

42. M. M. Ould Eleya and S. L. Turgeon, Food Hydrocolloids, 2000, 14, 29-40.

43. W. S. Gosal, A. H. Clark and S. B. Ross-Murphy, Biomacromolecules, 2004, 5, 2420-2429.

44. A. Clark, Functional properties of food macromolecules, 1998, 77.

45. M. Stading and A.-M. Hermansson, Food Hydrocolloids, 1991, 5, 339-352.

46. G. Agoda-Tandjawa, S. Durand, C. Gaillard, C. Garnier and J. L. Doublier, Carbohydrate Polymers, 2012, 87, 1045-1057.

47. M. Beaulieu, S. L. Turgeon and J.-L. Doublier, International dairy journal, 2001, 11, 961-967.

48. C. Ersch, I. ter Laak, E. van der Linden, P. Venema and A. Martin, Food Hydrocolloids, 2015, 44, 59-65.

49. A. Clark, G. Kavanagh and S. Ross-Murphy, Food Hydrocolloids, 2001, 15, 383-400.

50. P. B. Fernandes, Food Hydrocolloids, 1994, 8, 277-285.

51. M. Langton and A.-M. Hermansson, Food Hydrocolloids, 1992, 5, 523-539.

52. J. G. Montejano, D. D. Hamann and T. C. Lanier, Journal of Texture Studies, 1985, 16, 403-424.

53. W. Chantrapornchai and D. J. McClements, Food Hydrocolloids, 2002, 16, 467-476.

54. E. A. Foegeding, E. L. Bowland and C. C. Hardin, Food Hydrocolloids, 1995, 9, 237-249. 
Chapter 4

55. V. Urbonaite, S. van der Kaaij, H. de Jongh, E. Scholten, K. Ako, E. van der Linden and L. Pouvreau, Food Hydrocolloids, 2016, 56, 334-343.

56. S.-B. Lin, L.-C. Chen and H.-H. Chen, Journal of Food Process Engineering, 2011, 34, 1363-1379.

57. A. Okiyama, M. Motoki and S. Yamanaka, Food Hydrocolloids, 1993, 6, 493-501. 


\section{Chapter 5}

\section{Directed growth of protein fibrils}

\section{in the presence of cellulose}

microfibrils

\section{Submitted as:}

Jinfeng Peng, Vincenzo Calabrese, Julia Geurtz, Krassimir P. Velikov, Paul Venema, Erik van der Linden. Directed growth of protein fibrils in the presence of cellulose microfibrils. 


\begin{abstract}
In this study, we investigated the gelation of WPI fibrils in the presence of bacterial cellulose $(\mathrm{BC})$ microfibrils at $\mathrm{pH} 2$ upon prolonged heating. Rheology and microstructure were investigated as a function of $B C$ microfibril concentration. The presence of $B C$ microfibrils did not influence the gelation dynamics and resulting overall structure of the WPI fibrillar gel. The storage modulus and loss modulus of the mixed WPI-BC microfibril gels increased with increasing BC microfibril concentration, while the ratio between loss modulus and storage modulus remained constant. The WPI fibrils and BC microfibrils independently form two co-existing gel networks. Interestingly, near to the BC microfibrils we observed more aligned WPI fibrils, with individual WPI fibrils clearly distinguishable. The level of alignment of the WPI fibrils is dependent on the distance between BC microfibrils and WPI fibrils. This also is in line with our observation that with more BC microfibrils present, WPI fibrils are more aligned than in a WPI fibrillar gel without BC microfibrils. The large deformation response of the gels at different BC microfibril concentration and $\mathrm{NaCl}$ concentration is mainly influenced by the concentration of $\mathrm{NaCl}$, which affects the WPI fibrillar gel structures, changing form linear fibrillar to a particulate gel. The WPI fibrillar gel yields the dominant contribution to the gel strength.
\end{abstract}




\subsection{Introduction}

The ability of proteins to form fibrils under certain conditions has been suggested as a generic feature to all proteins. ${ }^{1,2}$ The subject has received considerable attention in the past decades. $^{3-14}$ Whey protein is a class of proteins frequently studied for fibril formation. ${ }^{15}$ A commercial system used for this purpose is whey protein isolate (WPI), containing a mixture of various types of whey protein. It is composed of beta-lactoglobulin $(\beta-\lg )$, alpha-lactalbumin ( $\alpha$-lac), and bovine serum albumin (BSA). ${ }^{16}$ Upon heating WPI at $80^{\circ} \mathrm{C}$ at $\mathrm{pH} 2$ and low ionic strength for several hours, $\beta$-lg was found to be the protein involved in fibril formation. ${ }^{17-19}$ In fact, the protein is first hydrolysed, due to the low $\mathrm{pH}$, to peptides and part of the peptides assemble into fibrils. ${ }^{12}$ Above a certain WPI concentration ( $6 \mathrm{wt} \%$ ), transparent gels consisting of these fibrils can be obtained. ${ }^{18}$ The fibrillar gels prepared from whey proteins by prolonged heating at $\mathrm{pH} 2$ have been studied previously with respect to their gel structure and rheological properties. ${ }^{13,18,20-28}$ However, little research has been performed on the formation and properties of fibrillar whey protein gels mixed with other fibrillar structures.

One of such fibrillar structures is bacterial cellulose (BC) microfibrils. This has recently received considerable interest in foods as a functional material due to its multifunctionality such as gelling, thickening, stabilizing and water binding abilities. ${ }^{29-35}$ Their health benefits include use as a dietary fibre and low-calorie food ingredient (i.e., fat substitute). This forms an added-value for use in foods. ${ }^{36-38}$ In addition, the BC microfibrils is highly pure in comparison to structures obtained from plant cellulose. ${ }^{39}$ The microfibrils of $B C$ are ribbon-shaped due to the interfibril hydrogen bonding and van der Waals attraction. These microfibrils have the tendency to agglomerate into a space-filling network and exhibit gel-like properties. ${ }^{40}$ The length of the mirofibrils is of the order of micrometer, while their width is of the order of nanometer. ${ }^{41}$ The research on BC microfibrils has mainly focused on its formation and production. ${ }^{42,43}$ Limited research has

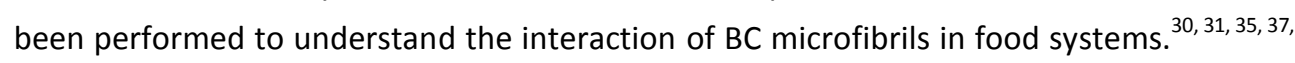
38

Recently, BC microfibril dispersions prepared from the well-known dessert in Philippines, named Nata de Coco, ${ }^{44}$ through high energy de-agglomeration has been characterized by Kuijk et al. ${ }^{40}$ and further modified with addition of a charged polymer by Veen et al. ${ }^{45,46}$ In 
the current study, we investigate mixed gels consisting of WPI fibrils and BC microfibrils as obtained from prolonged heating at $\mathrm{pH} 2$ of a WPI solution mixed with BC microfibrils. Our hypothesis was that WPI still forms fibrils in the presence of BC microfibrils under this condition, and that a WPI fibrillar gel is formed in co-existence with a microfibrillar BC gel. We characterized the rheological properties, microstructure and mechanical properties of the bi-fibrillar gels.

\subsection{Materials and Methods}

\subsubsection{Whey protein isolate (WPI) solution}

We followed the same procedure as Chapter 3 for the preparation of WPI solution at $\mathrm{pH} 2$. WPI (Bipro, Davisco, lot \# JE 198 -1-420, USA) powder was first dissolved in an $\mathrm{HCl}$ solution of $\mathrm{pH} 2$ with stirring at $4{ }^{\circ} \mathrm{C}$ overnight. The concentration of the WPI in the solution is about $15 \mathrm{wt} \%$. After dissolution, the $\mathrm{pH}$ of the WPI solution was adjusted to $\mathrm{pH} 2$ with a 6 $\mathrm{M} \mathrm{HCl}$ solution. Subsequently, the solution was filtered using a syringe filter (Hydrophilic PES $0.45 \mu \mathrm{m}$, Millipore Millex-HP) to remove undissolved protein. UV spectrophotometer (Cary 50 Bio, Varian) and a calibration curve of known WPI concentration at a wavelength of $278 \mathrm{~nm}$ was used to determine the protein concentration in the solution. The WPI stock solution was left at $4{ }^{\circ} \mathrm{C}$ before further sample preparation.

\subsubsection{Bacterial cellulose $(\mathrm{BC})$ microfibril dispersion}

The dispersion of 1 wt\% BC microfbrils was obtained from Unilever R\&D Vlaardingen, The Netherlands. The preparation procedure refers to Chapter 3 and more details can be found in literature. ${ }^{40,45}$

\subsubsection{WPI-BC bi-fibrillar gels}

We used the WPI stock solution and BC microfibril dispersion prepared above for mixture preparation. A solution of $\mathrm{pH} 2$ was used for dilution to reach the desired concentration ratios of WPI and BC microfibrils. The final WPI concentration in the mixtures was fixed at 9 wt\% and the BC microfibril concentrations were $0,0.05,0.1,0.2,0.3$ and 0.4 wt\%, respectively. For homogeneous mixing, all samples were stirred for 30 min prior to analysis. The $\mathrm{pH}$ of the final mixture was adjusted to $\mathrm{pH} 2$ using a $6 \mathrm{M} \mathrm{HCl}$ solution. For gel 
preparation, the mixtures were heated in a water bath (Haake Phoenix II C25P) from 20 to $80{ }^{\circ} \mathrm{C}$ with heating rate of $2{ }^{\circ} \mathrm{C} / \mathrm{min}$, holding at $80{ }^{\circ} \mathrm{C}$ for 10 hours and cooling from 80 to $20{ }^{\circ} \mathrm{C}$ with a cooling rate of $2{ }^{\circ} \mathrm{C} / \mathrm{min}$. After heating, the mixtures were left at $20{ }^{\circ} \mathrm{C}$ for further analysis.

\subsubsection{Differential Scanning Calorimetry (DSC)}

DSC was performed by placing about $50 \mathrm{mg}$ sample in a sealed stainless steel pan in the equipment (Diamond series DSC, Perkin Elmer, Pyris, USA). The measurement was performed by first equilibrating at $20{ }^{\circ} \mathrm{C}$ for $2 \mathrm{~min}$; afterwards, the sample was heated from $20^{\circ} \mathrm{C}$ to $100{ }^{\circ} \mathrm{C}$ at a heating rate of $10^{\circ} \mathrm{C} / \mathrm{min}$, held at $100{ }^{\circ} \mathrm{C}$ for $1 \mathrm{~min}$, and then cooled from $100^{\circ} \mathrm{C}$ to $20^{\circ} \mathrm{C}$ at a cooling rate of $10^{\circ} \mathrm{C} / \mathrm{min}$. After equilibrating at $20^{\circ} \mathrm{C}$ for 2 min, the sample was again heated from $20^{\circ} \mathrm{C}$ to $100^{\circ} \mathrm{C}$ at a heating rate of $10^{\circ} \mathrm{C} / \mathrm{min}$. All samples were measured in duplicate. The software of Pyris (Perkin Elmer) was used for data analysis.

\subsubsection{Small deformation rheology}

The WPI-BC microfibril mixtures were stirred at room temperature for one hour prior to measurement. Then, the samples were transferred using a pipette with a cut-off tip to a stress-controlled rheometer (MCR 302, Anton Paar). Measurement was performed using sandblasted concentric cylinder geometry (CC17/TI/S-SN38492). To prevent evaporation, the sample was covered with a thin layer of paraffin oil and a solvent trap was also used. The development of storage modulus $G^{\prime}$ and loss modulus $G^{\prime \prime}$ during gel formation was monitored at a frequency of $1 \mathrm{~Hz}$ and strain of $0.1 \%$. The temperature during the gel formation was first increased from 20 to $80^{\circ} \mathrm{C}$ at a heating rate of $2{ }^{\circ} \mathrm{C} / \mathrm{min}$, held at $80^{\circ} \mathrm{C}$ for 10 hours, decreased from 80 to $20^{\circ} \mathrm{C}$ at a cooling rate of $2^{\circ} \mathrm{C} / \mathrm{min}$, and then held at 20 ${ }^{\circ} \mathrm{C}$ for $30 \mathrm{~min}$. Afterwards, a frequency sweep was determined at a strain of $0.1 \%$ and frequency increasing from 0.01 to $100 \mathrm{~Hz}$ within $30 \mathrm{~min}$ at $20{ }^{\circ} \mathrm{C}$. Additionally, a strain sweep was also performed at a frequency of $1 \mathrm{~Hz}$ and strain increasing from 0.01 to $1000 \%$ within $30 \mathrm{~min}$ at $20^{\circ} \mathrm{C}$. 


\subsubsection{Confocal laser scanning microscopy (CLSM)}

$1 \mathrm{ml}$ of sample is transferred to an Eppendorf. $5 \mu \mathrm{l}$ of $0.2 \%$ Rhodamine B and $10 \mu \mathrm{l}$ of $0.05 \%$ Calcofluor white were added to stain the WPI and BC microfibrils, respectively. The sample was vortexed for $10 \mathrm{~s}$ for homogeneous mixing. Sample with a volume of $125 \mu \mathrm{l}$ was transferred into CLSM cuvettes (Gene Frame $125 \mu \mathrm{l}$, Thermo Scientific). The cuvettes were sealed by gently gluing a cover glass (Menzel-glaser, Thermo Scientific, Germany) on the top. The cuvettes were then transferred and heated in a water bath (Haake Phoenix II $\mathrm{C} 25 \mathrm{P}$ ) for gel preparation. The temperature was increased from 20 to $80{ }^{\circ} \mathrm{C}$ at a heating rate of $2{ }^{\circ} \mathrm{C}$, held at $80^{\circ} \mathrm{C}$ for 10 hours and then decreased from 80 to $20^{\circ} \mathrm{C}$ at a cooling rate of $2{ }^{\circ} \mathrm{C}$. Samples were kept at room temperature for one day before imaging. CLSM image analysis was performed on a Zeiss LSM 510 META microscope (Zeiss, Germany) equipped with an Axiovert 200M inverted microscope using an oil immersion objective (Plan-Apochromat $63 x / 1.4$ oil DIC). The excitation wavelength of Rhodamine $B$ and Calcofluor white are $543 \mathrm{~nm}$ and $405 \mathrm{~nm}$. The emission wavelength of Rhodamine $B$ and Calcofluor white are $560 \mathrm{~nm}$ and $420 \mathrm{~nm}$.

\subsubsection{Scanning Electron Microscopy (SEM)}

For image analysis of BC dispersion using SEM, a small droplet of the sample was put on a hollow copper rivet and rapidly frozen in liquid ethane. The rivets were placed in a cryosample holder in liquid nitrogen and hereafter transferred to the cryo-preparation system (MED 020/ VCT 100, Leica, Vienna, Austria) onto the sample stage. Subsequently, the samples were fractured, freeze dried for 10 minutes and then sputter coated with a layer of $10 \mathrm{~nm}$ tungsten at the same environmental conditions. The samples were cryo-shielded and transferred into the field emission scanning microscope (Magellan 400, FEl, Eindhoven, The Netherlands) onto the sample stage for further analysis.

Gels were prepared in pre-lubricated $20 \mathrm{ml}$ syringes for SEM imaging. The gels were cut into five pieces which are $1 \mathrm{~cm}$ in diameter and $1.3 \mathrm{~cm}$ in height and placed in $2.5 \%(\mathrm{v} / \mathrm{v})$ glutaraldehyde solution for $8 \mathrm{hrs}$ for crosslinking of the proteins. The excess glutaraldehyde was removed by placing the gel pieces in MilliQ water with gently rotating overnight. The water was replaced gradually by $10,30,50,70$, and $100 \%$ acetone. The sample was gently rotated for 1 hour for each acetone concentration. Finally, the samples 
were left in $100 \%$ acetone. Subsequently, the sample pieces were dried using critical point drying (CPD 300, Leica, Vienna, Austria) and then were fractured and attached onto SEM sample holders using Carbon Conductive Cement (Leit-C, Neubauer Chemicalien, Germany). The samples were stored overnight under vacuum for removal of all the solvent from the adhesive. After sputter coating with a $10 \mathrm{~nm}$ thick layer of iridium (SCD 500 , Leica, Vienna, Austria) the fractured surfaces were analyzed on a scanning electron microscope (Magellan 400, FEI, Eindhoven, the Netherlands).

\subsubsection{Large deformation rheology}

Gels were prepared in $20 \mathrm{ml}$ syringes (BD plastipak, Spain) which were pre-lubricated using paraffin oil. Cylindrical gel samples $(20 \mathrm{~mm}$ in height and $20 \mathrm{~mm}$ in diameter) were obtained using a wire cutter. The fracture properties of the gels were measured by performing a uniaxial compression test on a Texture Analyser (TA-XT plus, Stable Micro Systems, Godalming, U.K) fitted with a $50 \mathrm{~kg}$ load cell and a cylindrical plastic probe of 75 $\mathrm{mm}$ in diameter. The gels were compressed to $80 \%$ deformation at a rate of $1 \mathrm{~mm} / \mathrm{s}$ at room temperature. Four measurements were performed for each sample. The true stress, true strain and Young's modulus were calculated on the basis of the method described elsewhere. $^{47}$

\subsection{Results and discussion}

\subsubsection{Macroscopic stability}

Pure WPI solution is transparent at $\mathrm{pH}$ 2. Adding BC microfibrils to the solution leads to a turbid mixture (Figure 1). The turbidity increases with increase in BC microfibril concentration due to the presence of thicker and long BC microfibril bundles. ${ }^{40}$ The mixtures showed macroscopically homogeneous and were stable at all BC microfibril concentrations. Upon prolonged heating at $\mathrm{pH} 2$, pure WPI solution forms linear and long fibrils. $^{18,22,23}$ At concentration of WPI above $6 \mathrm{wt} \%$, a transparent gel with a threedimensional network consisting of long fibril aggregates is formed, ${ }^{18,21,23,25,48}$ as also observed in Figure 1 at 9 wt\% pure WPI. WPI contains a mixture of proteins with $\beta$ lactoglobulin $(\beta-\mathrm{lg})$ as the major protein $(\sim 60 \%)$. Bolder et al. ${ }^{17-19}$ reported that $\beta$-lg is the only protein involved in fibril formation under the conditions used here. In the presence of 
BC microfibrils, the WPI gels formed are translucent and become turbid upon increasing $\mathrm{BC}$ microfibril concentration. The $\mathrm{BC}$ microfibrils do not gel upon prolonged heating at $\mathrm{pH}$ 2, as confirmed in Chapter 3.

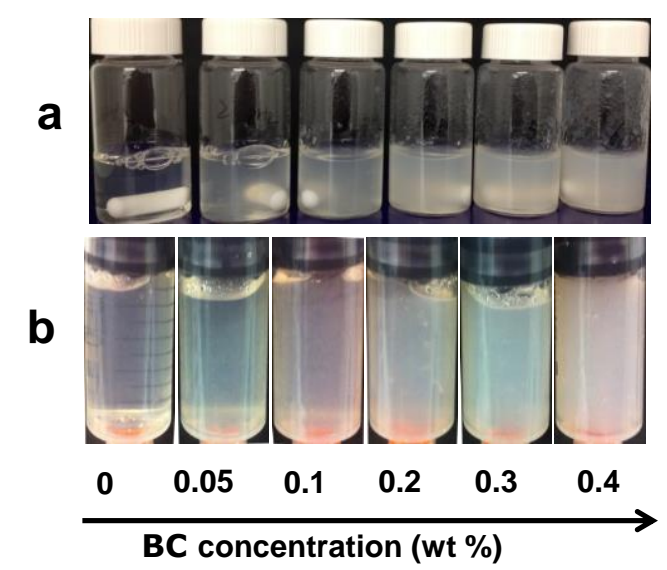

Figure 1 Images of WPI-BC microfibril mixtures as a function of $\mathrm{BC}$ microfibril concentration at pH 2 (a) and the corresponding heat-induced gels (b). All Samples contain 9 wt\% WPI and BC microfibril concentrations range from $0,0.05,0.1,0.2$, and 0.3 to $0.4 \mathrm{wt} \%$, as indicated below the image.

\subsubsection{Differential Scanning Calorimetry (DSC)}

The $\mathrm{BC}$ microfibrils did not show endothermic peaks on heating at $\mathrm{pH} 2$. The WPI solution exhibited an endotherm peak upon heating due to denaturation, the peak temperature is around $80^{\circ} \mathrm{C}$. Adding BC microfibrils, to WPI solution gave a similar endothermic peak to that of the pure WPI solution, regardless of the concentrations of BC microfibrils used. When reheating the samples, no peaks were observed, indicating the irreversible denaturation of WPI. The thermal behaviour of whey proteins has been reported to be dominated by the behaviour of beta-lactoglobulin ( $\beta-\mathrm{lg})$. The denaturation temperature of WPI found here at low $\mathrm{pH}$ is in agreement with literature, ${ }^{49,50}$ suggesting that the addition of BC in WPI solution did not influence the denaturation temperature of WPI. This is likely to denote the absence of specific interactions ${ }^{51}$ between WPI and BC microfibrils; WPI denatures in the mixtures with $B C$ microfibrils the same way as without the $B C$ microfibrils present. 


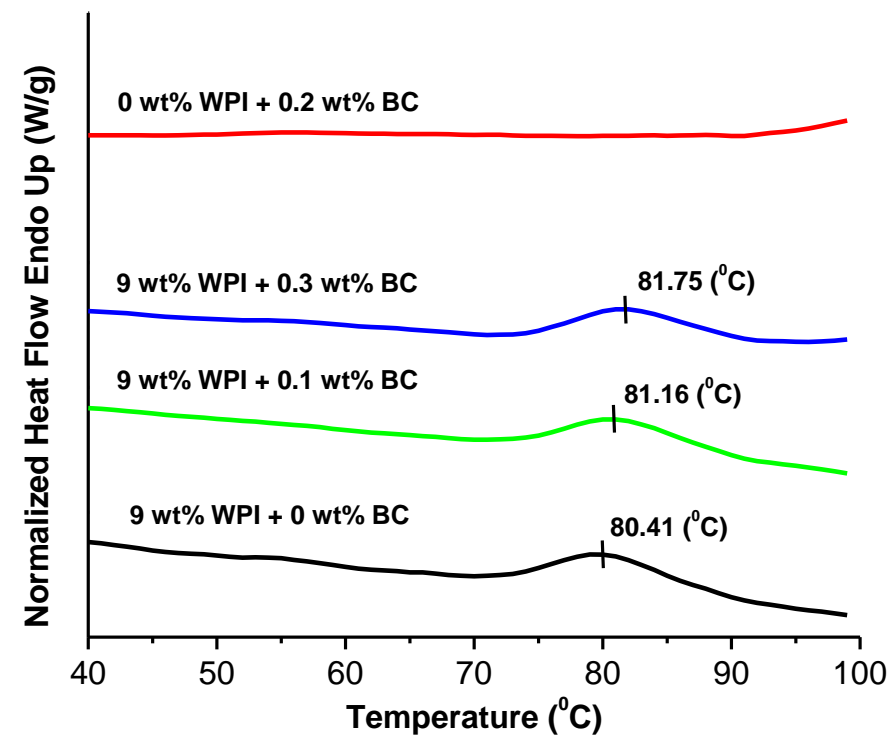

Figure 2 DSC thermograms for pure WPI, pure BC microfibrils and mixed WPI-BC microfibril samples. Concentrations are indicated above the lines.

\subsubsection{Rheological properties}

For the sample containing only $0.3 \mathrm{wt} \%$ pure BC microfibrils (Figure 3), the storage modulus $G^{\prime}$ and loss modulus $G^{\prime \prime}$ remained constant over time, despite the changes in temperature, indicating that the rheological properties of $\mathrm{BC}$ microfibrils are stable against the temperature change at $\mathrm{pH}$ 2. In pure WPI gels (9 wt\%), the $\mathrm{G}^{\prime}$ and $\mathrm{G}^{\prime \prime}$ started with a small value. When temperature reached $80^{\circ} \mathrm{C}$, both $\mathrm{G}^{\prime}$ and $\mathrm{G}^{\prime \prime}$ underwent a sudden increase, indicating the start of WPI gelation. The $\mathrm{G}^{\prime}$ and $\mathrm{G}^{\prime \prime}$ reached a constant value after holding at $80^{\circ} \mathrm{C}$ for 10 hours. This is in agreement with Gosal et al. ${ }^{20}$, who has studied the gelation of $\beta$-lg at $\mathrm{pH} 2$ upon prolonged heating and such sudden increase is generally recognized as a typical sol-gel transitions of biopolymer solutions. ${ }^{52}$ Upon temperature decreasing to $20^{\circ} \mathrm{C}$, the $\mathrm{G}^{\prime}$ and $\mathrm{G}^{\prime \prime}$ continued to increase. A slight decrease of the $\mathrm{G}^{\prime}$ and G' was observed prior to further increase, this has been also observed in the study of Gosal et al. ${ }^{20}$ and was attributed to an artefact resulted from the slippage of the gel samples after prolonged heating at an elevated temperature, which was most likely to be 
caused by the creep of the paraffin oil between the gel surface and the measuring cup. This creep of the parrafin oil could be caused by gel shrinkage (syneresis). At temperature of $20^{\circ} \mathrm{C}$, the $\mathrm{G}^{\prime}$ and $\mathrm{G}^{\prime \prime}$ values of all gels remained constant.

Upon addition of BC mirofibrils to a WPI solution, all samples have an initial $\mathrm{G}^{\prime}$ that is higher than G'. The G' values increase with increasing BC microfibril concentration. This is in line with the gel-like structure of BC microfibrils, reported previously. ${ }^{40}$ We observed a behaviour of the $G^{\prime}$ and $G^{\prime \prime}$ development over time similar to that of the pure WPI sample, regardless of the $B C$ microfibril concentration. This suggests that the presence of $B C$ microfibrils does not influence the gelation time and gelation temperature of WPI. Plotting the $G^{\prime}$ and $G^{\prime \prime}$ as a function of BC microfibril concentration (Figure 4a), we observe an increase in $\mathrm{G}^{\prime}$ and $\mathrm{G}^{\prime \prime}$ upon increasing the $\mathrm{BC}$ microfibril concertation. Interestingly, the increase of $\mathrm{G}^{\prime}$ and $\mathrm{G}^{\prime \prime}$ as a function of $\mathrm{BC}$ microfibril concentration shows a similar trend, and this is further confirmed by plotting the tangent $\delta$ as a function of BC concentration (Figure $4 \mathrm{~b}$ ). Here we found that the value of tangent $\delta$ showed only a slight increase with increase of BC microfibril concentration. Since BC microfibrils are non-gelling agents upon heating, this result implies that the rheological properties of the mixed gel are mainly dominated by WPI and that the gel structure in the WPI-BC microfibril gel is the same as that of the pure WPI gel structure. ${ }^{53}$ We realise that the influence of the $\mathrm{BC}$ microfibril gel on the mixed gels might be too small to be reflected in rheological properties in comparison to the strong WPI gel. Therefore we also refer to the microscopic structural characterisations.

When applying a frequency sweep (Figure 5), the $G^{\prime}$ and $G^{\prime \prime}$ of the pure BC mirofibrils sample showed an independency of frequency up to $\sim 10 \mathrm{~Hz}$. In contrast to the pure BC microfibril gel, the pure WPI gel showed an independency of frequency up to $\sim 30 \mathrm{~Hz}$. With addition of BC microfibrils into WPI gel, the $G^{\prime}$ and $G^{\prime}$ show the same trend to that of pure WPI gel upon increasing frequency, implying that the rheological properties of the WPI-BC microfibril gels are dominated by the WPI gel structure. A strain sweep (Figure 6) was performed to confirm whether adding BC microfibrils to WPI gels affects the linear viscoelastic region (LVR). The results showed that adding BC microfibrils slightly leads to structure breakdown at a lower strain value, i.e. that addition of $\mathrm{BC}$ microfibrils makes the gels slightly more brittle. 
The rheological properties of the WPI-BC microfibril gels at $\mathrm{pH} 2$ showed a comparable behaviour to that of the pure WPI gel, indicating that the presence of BC microfibrils did not significantly influence the WPI gel formation and that the WPI gel dominates the rheological properties in the mixed gels. This is probably because of the relatively low concentrations of BC microfibrils in comparison to the high concentration of WPI. Increasing the BC microfibril concentration did result in a higher storage modulus and loss modulus of the gels. In protein-polysaccharide gels this effect was often observed and attributed to segregative phase separation. ${ }^{54-56}$ However, according to the macroscopic stability of the WPI-BC mixtures (Figure 1), it is unlikely that phase separation occurred. It would be more likely due to the water becoming preferably situated in the phase of $\mathrm{BC}$, thereby effectively concentrating the protein phase. ${ }^{57,58}$ 

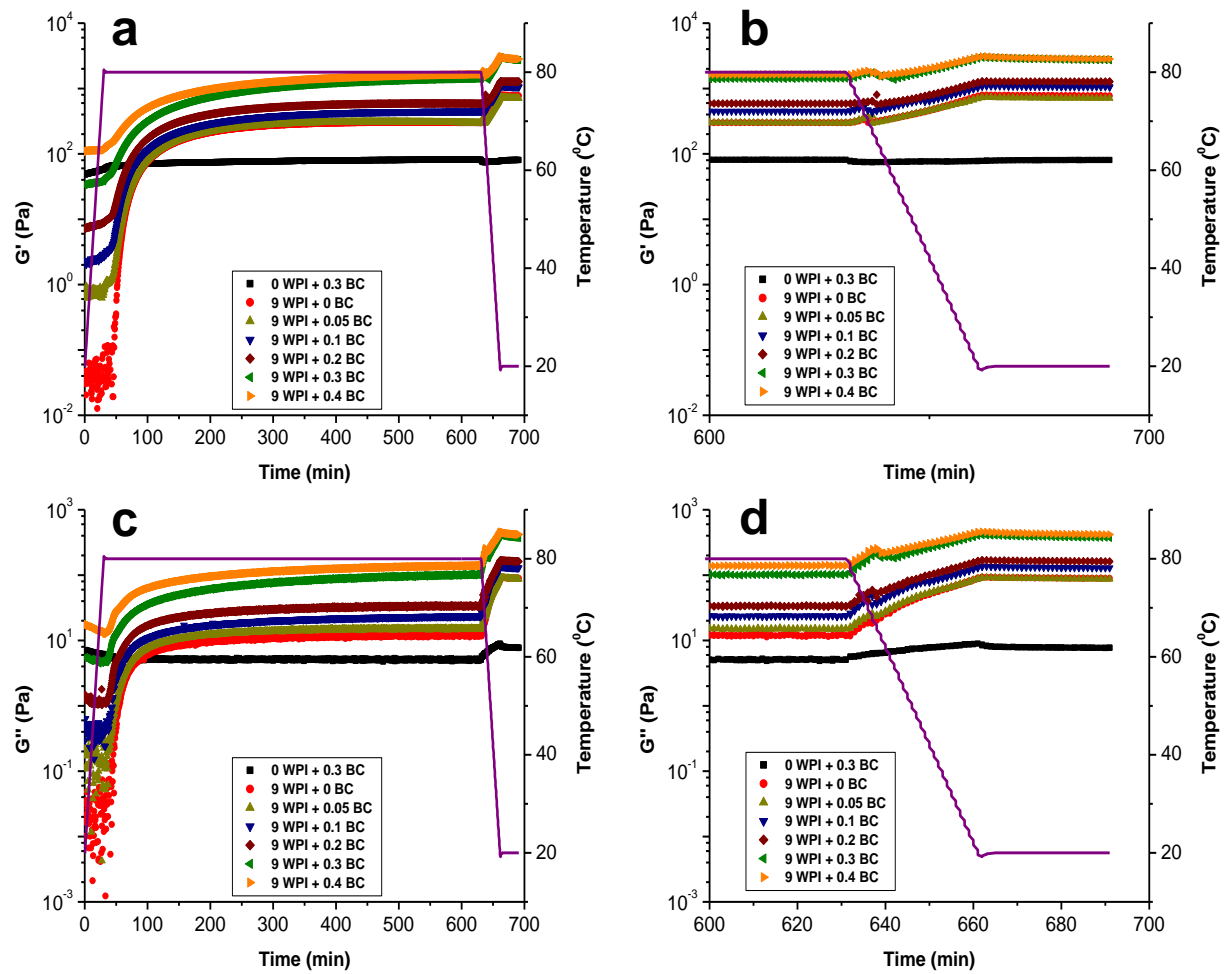

Figure 3 The storage modulus G' and loss modulus G" development of WPI-BC microfibril gels at pH 2 during gel formation. (a) and (c) show the G' (a) and G' (c) development of the samples as a function of time at a strain of $0.1 \%$ and frequency of $1 \mathrm{~Hz}$. (b) and (d) show the enlargement of $\mathrm{G}^{\prime}$ (c) and G" (d) between the time period of $600 \mathrm{~min}$ to $691 \mathrm{~min}$. The purple line represents the temperature profile. All samples containing a fixed WPI concentration of 9 wt\% and BC microfibril concentration varying from $0,0.05,0.1,0.2,0.3$, to $0.4 \mathrm{wt} \%$. 

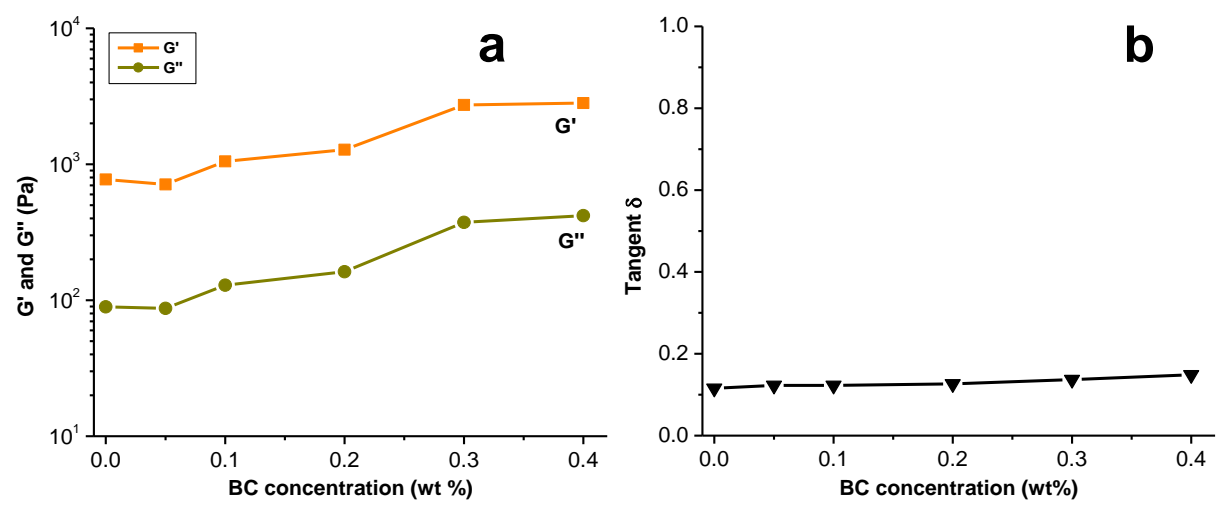

Figure 4 Storage modulus G' and loss modulus G" (a) and tangent $\delta$ (b) of WPI-BC microfibril gels as a function of BC microfibril concentration at a fixed 9 wt\% WPI. The values of $G^{\prime}$ and $G^{\prime \prime}$ are taken from the last point after holding the samples at $20^{\circ} \mathrm{C}$ for $30 \mathrm{~min}$. Lines are used as a guide to the eye.
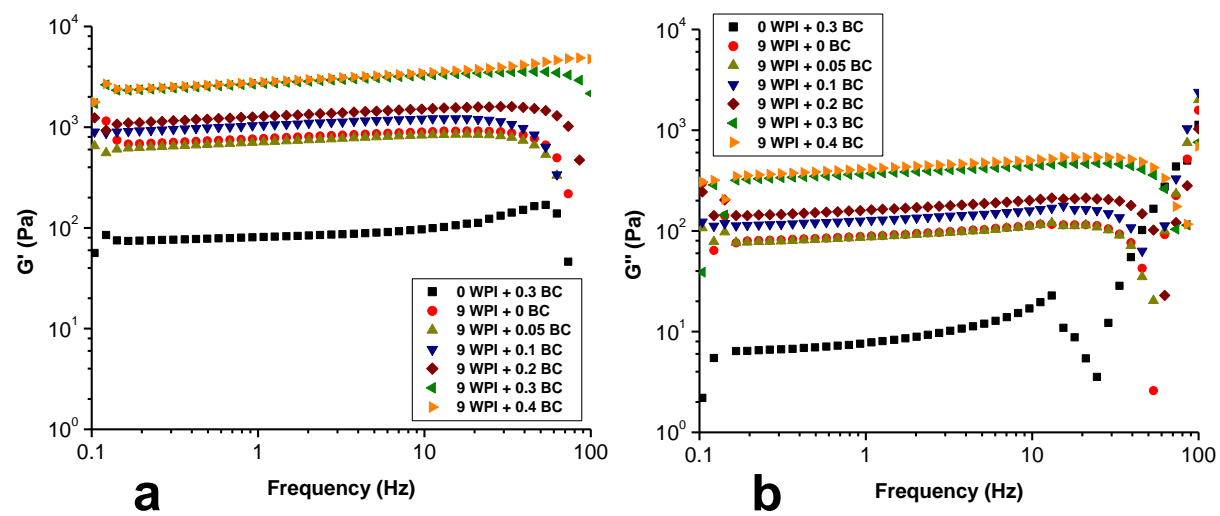

Figure 5 The storage modulus G' (a) and loss modulus G" (b) as a function of frequency at a strain of $0.1 \%$. All samples contain the same WPI concentration of 9 wt\% and BC microfibril concentrations ranging from $0,0.05,0.1,0.2,0.3$, to $0.4 \mathrm{wt} \%$. 


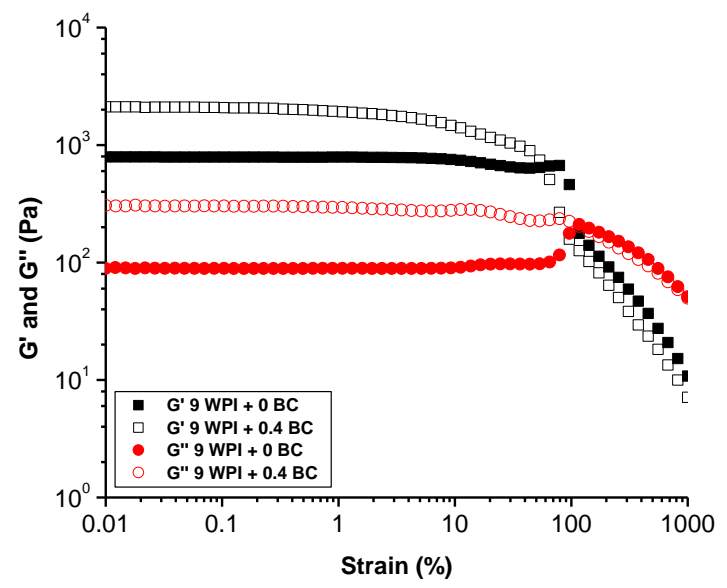

Figure 6 The storage modulus $\mathrm{G}^{\prime}$ and loss modulus $\mathrm{G}^{\prime \prime}$ as a function of strain at a frequency of $\mathbf{1 H z}$ for samples of 9 wt\% pure WPI fibrillar gel and bi-fibrillar gel containing $9 w t \%$ WPI and 0.4 wt\% BC microfibrils.

\subsubsection{Confocal laser scanning microscopy (CLSM)}

To investigate the WPI-BC microfibril gel microstructure, CLSM images were taken after gel formation. Figure 7 shows the CLSM images as a function of BC microfibril concentration. Pure BC microfibrils exhibited an inhomogeneous microstructure (Figrue 7). Flocs of $B C$ microfibril bundles are visible in the CLSM images, in line with the study of Kuijk et al., ${ }^{40}$ where they have shown that the BC microfibril dispersion is composed of fibril bundles, flocs and voids, resulting in highly heterogeneous structure. Pure WPI forms a homogeneous gel at pH 2 consisting of linear fibril aggregates with sizes too small to be visible in $\mathrm{CLSM}^{13,18,21,23,25}$ as confirmed in Figure 7. The network of BC microfibrils must become denser with increasing $B C$ microfibril concentrations in the mixed gel, but this cannot be concluded from CSLM images. The two gel structures do not seem to influence one another. Phase separation was not observed in the CLSM images. The signal for BC microfibrils was too weak to be clearly visualized in WPI gels, in contrast to the pure BC microfibrils sample. This could be explained by the large amount of protein particles surrounding, covering or absorbing on the surface of the $B C$ microfibrils. We still observe 
the $\mathrm{BC}$ microfibrils present in the overlay images, showing as the darker areas. This is also confirmed by the image from the transmission channel (not shown here) of the CLSM.

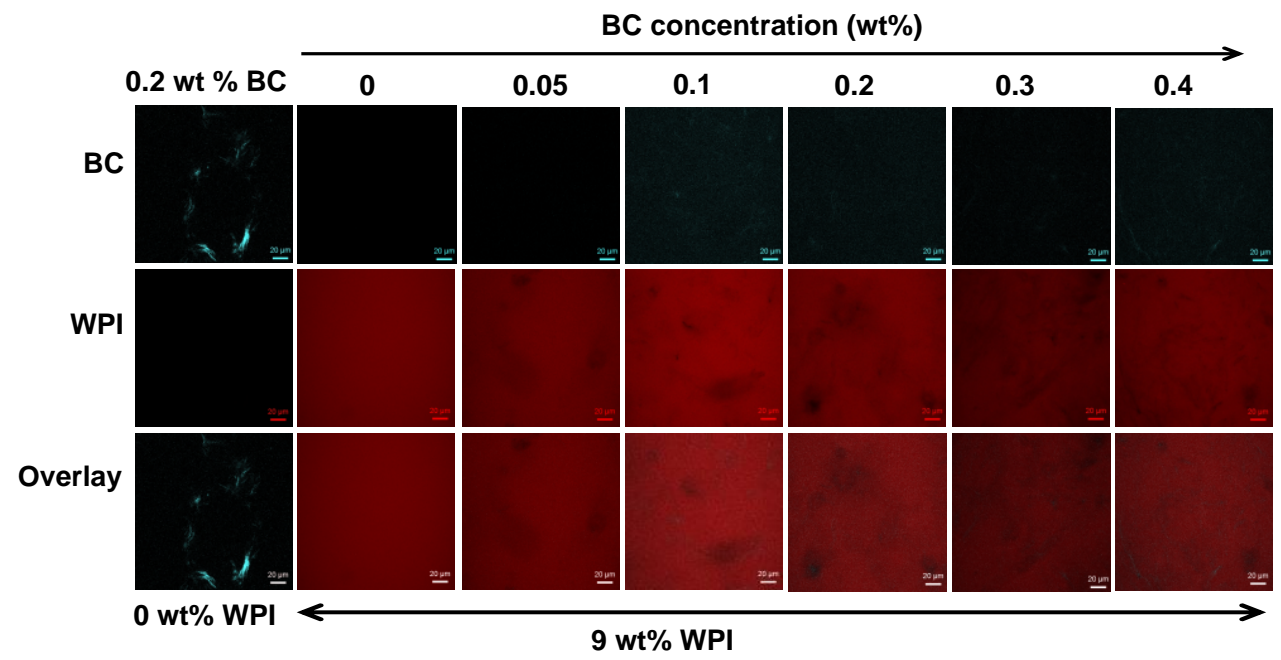

Figure 7 CLSM images of WPI-BC microfibril gels containing 9 wt\% WPI (shown in red) and BC microfibril (shown in green) concentrations of $0,0.05,0.1,0.2,0.3$ and $0.4 w t \%$. On the top of the images show the BC microfibril concentrations (wt\%) and at the bottom show WPI concentrations (wt\%). Images from the BC microfibril channel, WPI channel and the overlay image are shown. The scale bar corresponds to $20 \mu \mathrm{m}$.

\subsubsection{Scanning electron microscopy (SEM)}

SEM images were taken to obtain the details of the gel morphology. Images were taken at different magnifications and different locations within the sample. In particular, we focused on sample area where mainly WPI gel network is shown and area where both WPI and $\mathrm{BC}$ are present.

From Figure 8a, one can see that the pure WPI gels formed at pH 2 shows a smooth and homogeneous gel structure. With addition of BC microfibrils, we observed two gel structures overlapping in the sample, i.e. an homogeneous WPI gel and BC microfibril gel spreading over the system without much influence on the WPI gel. The overall gel structure becomes inhomogeneous and the inhomogeneity is increased with increase in $\mathrm{BC}$ microfibril concentration due to the presence of $\mathrm{BC}$ microfibril bundles. When focused 
on an area where only a WPI gel network is present, like that in a pure WPI gel, a lot of thin linear aggregates composed of peptides ${ }^{12}$ are observed, corresponding to the fibril WPI based structures. When comparing the WPI gel structure at different BC microfibril concentrations to the structure of the pure WPI gel, as shown in Figure $8 \mathrm{~b}$ at different magnifications, we observe that the WPI gels formed in the presence of BC microfibrils reveal a better aligned WPI fibrillar gel network. It is clearly shown that the fibrils are much finer and aligned next to one another in the direction parallel to that of the $B C$ microfibrils, in contrast to the more random oriented fibrillar structures in a pure WPI gel. Upon increasing BC microfibril concentration, the alignment of the fibrils becomes more pronounced. When zooming in on the area where both WPI fibrils and BC microfibrils are present (Figure $8 \mathrm{c}$ ), we observe the WPI fibrils parallel to the $B C$ microfibrils. The $B C$ microfibril gel structure did not show significant changes as a function of its concentration in WPI fibrillar gels. In Figure 9, images taken in a different location in the sample are shown. We observed the same WPI fibril alignment along the BC microfibrils. The alignment of WPI fibrils is enhanced with increase of BC microfibril concentration in the WPI gel.

Interestingly, the extent of alignment of WPI fibrils varies within the sample, i.e., in some areas, the WPI fibrils structure show similar to the pure WPI fibril structure, whereas in other areas, the WPI fibrils show aligned to each other. It seems that the alignment depends on how far a specific area of WPI is from the neighbouring BC microfibrils. To confirm this, the effect of the distance between WPI and BC fibrils on the WPI fibril structure is investigated. To do this, we fixed the area where $B C$ microfibrils are present (shown in green in Figure 10), and imaged the structure of the neighbouring WPI fibrils (shown in red) as a function of their distance to BC microfibrils, as shown in Figrue 10. Results showed that in the area where $B C$ microfibrils are present, the neighbouring WPI fibrils are better aligned than the WPI fibrils that are farther away from the BC microfibrils. In other words, the closer the WPI is located to BC microfibrils, the better aligned the WPI fibrils are. When there is no $B C$ microfibrils around the WPI or the BC microfibrils are far enough from the WPI, the WPI fibril structure looks similar to that of a pure WPI fibrillar gel.

The morphology of the gels from SEM reveals that adding BC microfibrilsto WPI fibril forming solutions resulted upon heating in the formation of a bi-fibrillar gel structure. 
Interestingly, BC microfibrils serve as a director for WPI fibril formation, leading to formation of WPI fibrils aligned next to one another when in the neighbourhood of $B C$ microfibers. Increasing the BC microfibril concentration enhanced the alignment of WPI fibrils next to one another in the neighbourhood of the BC microfibrils.

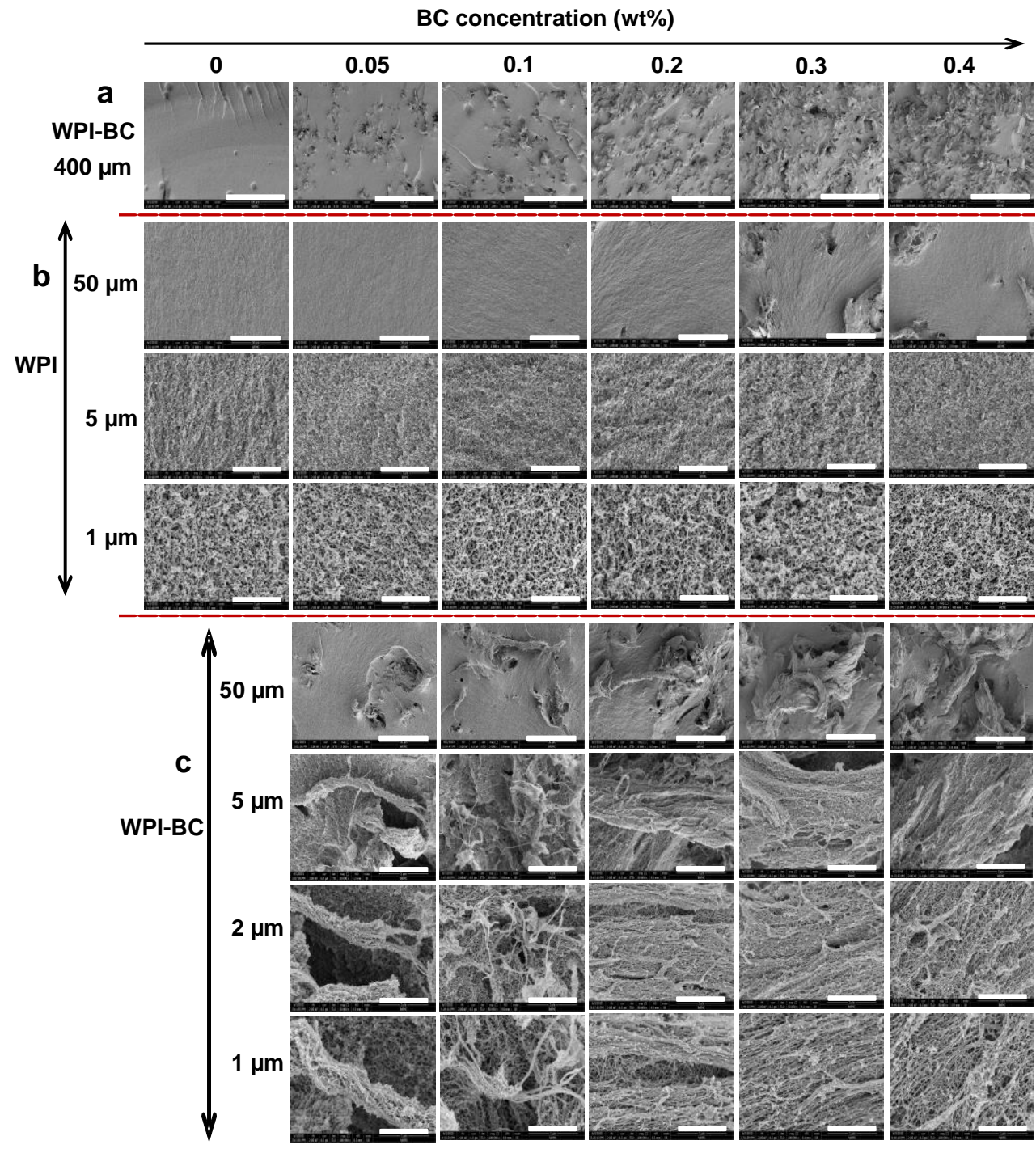

Figure 8 SEM images of WPI-BC microfibril gels containing 9 wt\% WPI and BC microfibril concentration of $0,0.05,0.1,0.2,0.3$ and $0.4 \mathrm{wt} \%$. (a) shows the overall structure of the gels, (b) 
shows the structure at locations where mainly WPI is present, and (c) shows the structure at locations where both WPI and BC microfibrils are present. Scale bars correspond to $400 \mu \mathrm{m}, 50 \mu \mathrm{m}$, $5 \mu \mathrm{m}, 2 \mu \mathrm{m}$, and $1 \mu \mathrm{m}$.

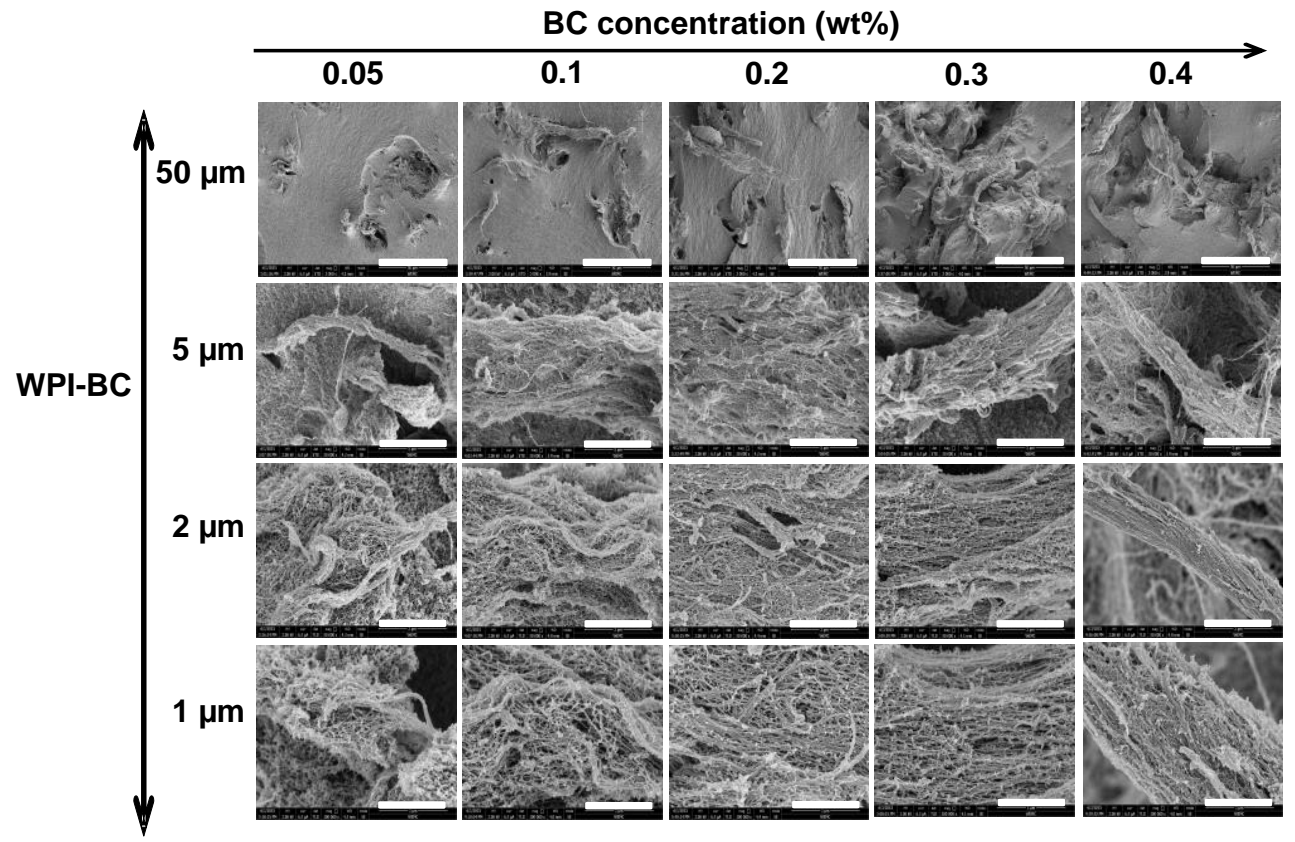

Figure 9 SEM images of WPI-BC gels containing 9 wt\% WPI and BC concentration of $0,0.05,0.1,0.2$, 0.3 and 0.4 wt\% BC. Images show the structure at locations where both WPI and BC microfibrils are present. Scale bars corresponds to $50 \mu \mathrm{m}, 5 \mu \mathrm{m}, 2 \mu \mathrm{m}$, and $1 \mu \mathrm{m}$. 


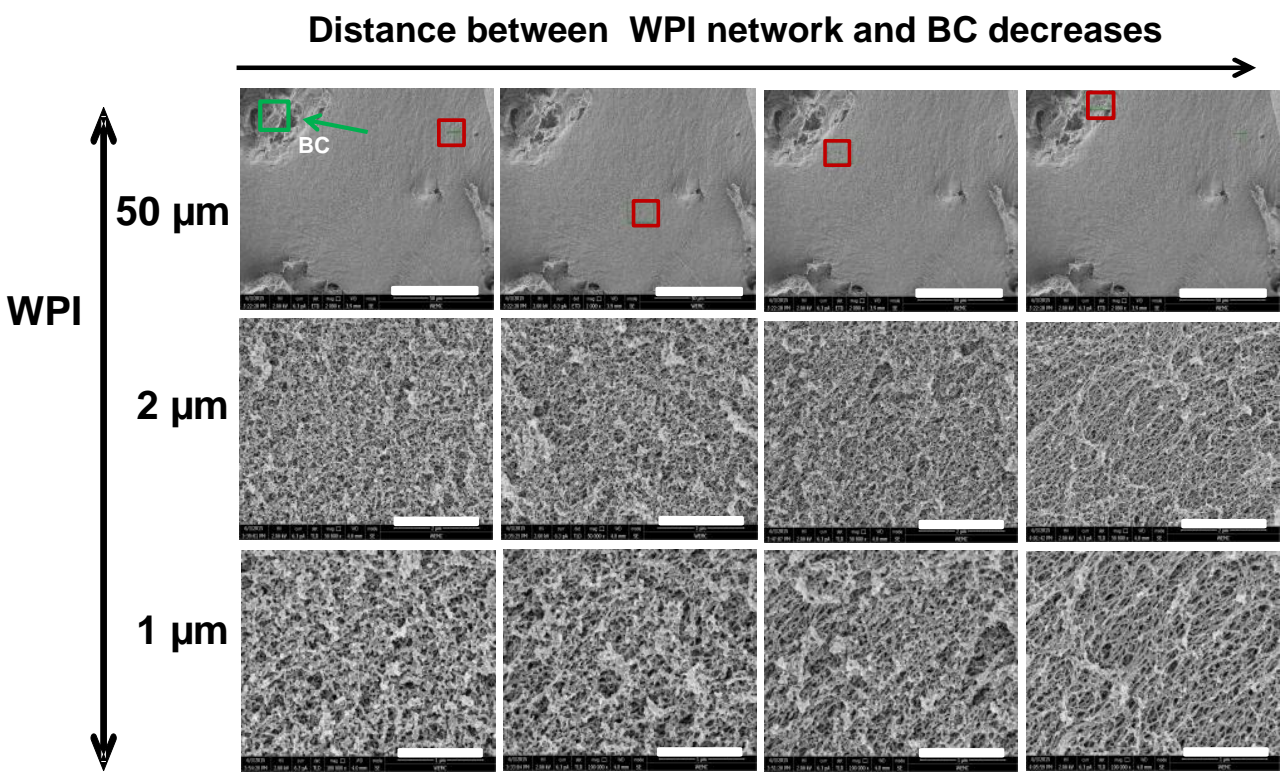

Figure 10 SEM images showing the WPI structure (red square) as a function of the distance to the position where BC microfibrils are present. From left to right, the distance between WPI and BC microfibrils decreases. Scale bars correspond to $50 \mu \mathrm{m}, 2 \mu \mathrm{m}$, and $1 \mu \mathrm{m}$.

\subsubsection{Large deformation properties}

Large deformation rheological properties were measured to investigate the effect of $B C$ microfibrils on the fracture properties of the WPI gel. Moreover, $\mathrm{NaCl}$ was also added to the mixture, the concentrations used are 0, 50, 100 and $200 \mathrm{mM}$. The dependency of WPI gel structure on ionic strength at $\mathrm{pH} 2$ has been studied previously by several authors. ${ }^{13,23 \text {, }}$ $24,26,28$ WPI gels formed at $\mathrm{pH} 2$ were found to be transparent up to a salt concentration of $100 \mathrm{mM} .^{28}$ With increase in salt concentration, the electrostatic repulsion is reduced and the fibrils formed become shorter and more flexible. ${ }^{13,14,23,26,28}$ This further results in different gel structures. ${ }^{14,26}$ However, structure of BC microfibrils are stable against salt concentration, as concluded from the TEM and CLSM images (results not shown). No significant difference of $B C$ microfibril microstructure was observed. From the gel macroscopic images shown in Figure 11, the appearance of gels from pure WPI changed from transparent to more opaque with increase in salt concentration, which is in line with 
previous studies. ${ }^{28}$ Increasing BC microfibril concentration resulted in more opaque gels as discussed above.

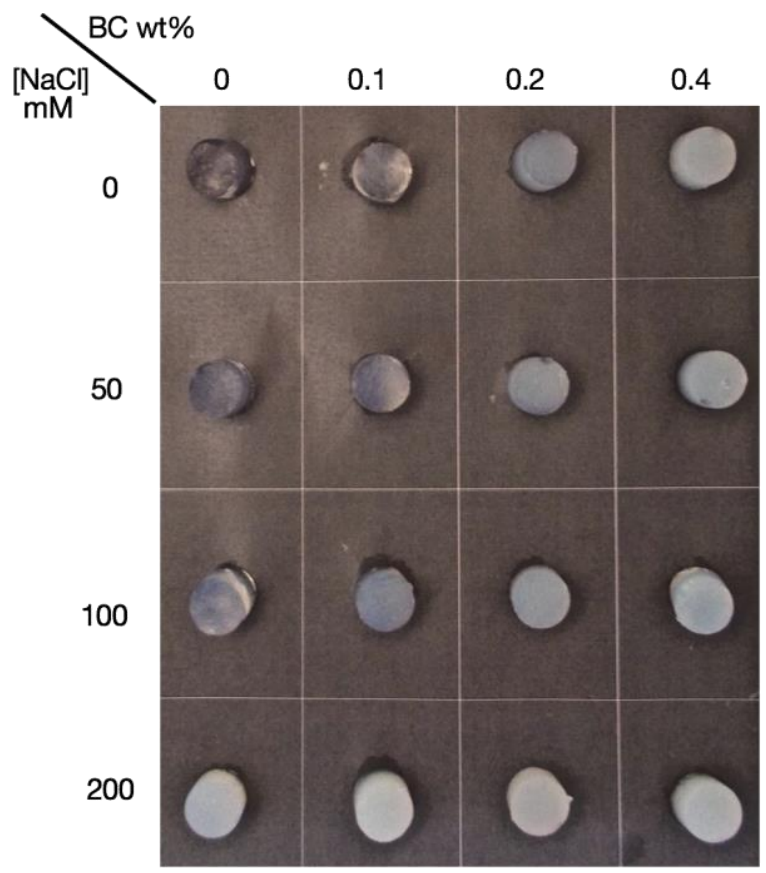

Figure 11 Macroscopic images of WPI-BC microfibril gels prepared at $\mathrm{NaCl}$ concentration of 0,50 , 100 , and $200 \mathrm{mM}$ after 1 day of the preparation. All samples containing the same WPI concentration of $9 w t \%$ and $B C$ microfibril concentrations of $0,0.1,0.2$, and $0.4 w t \%$ as indicated on the top of the images. The $\mathrm{NaCl}$ concentrations $(\mathrm{mM})$ are indicated on the left side of the images.

As shown in Figure $12 \mathrm{~A}$, at $0 \mathrm{mM} \mathrm{NaCl}$, upon addition of $\mathrm{BC}$ microfibrils, the WPI-BC microfibril gels showed a similar correlation for true stress as a function of true strain to that of the pure WPI gel. All gels fractured at almost the same strain upon compression. However, the fracture stress is increased in the presence of $B C$ microfibrils, but there is no significant difference for different BC microfibril concentrations (Figure $13 \mathrm{~B}$ ). This corresponds to small deformation properties as discussed above, where we found that addition of $\mathrm{BC}$ microfibrils increases the gel strength. With increasing $\mathrm{NaCl}$ concentration, the fracture stress of pure WPI gels increased, whereas the fracture strain decreased. Higher $\mathrm{NaCl}$ concentrations yield a coarser gel structure. In the mixed WPI-BC microfibril 
gels, with increase in $\mathrm{NaCl}$ concentration, the gels fracture at lower strain and higher stress. In general, the WPI-BC microfibril gels showed similar characteristics upon compression to that of a pure WPI gel at the same $\mathrm{NaCl}$ concentration. The presence of $\mathrm{NaCl}$ only influences the WPI gel structure, not the $\mathrm{BC}$ microfibril structure.
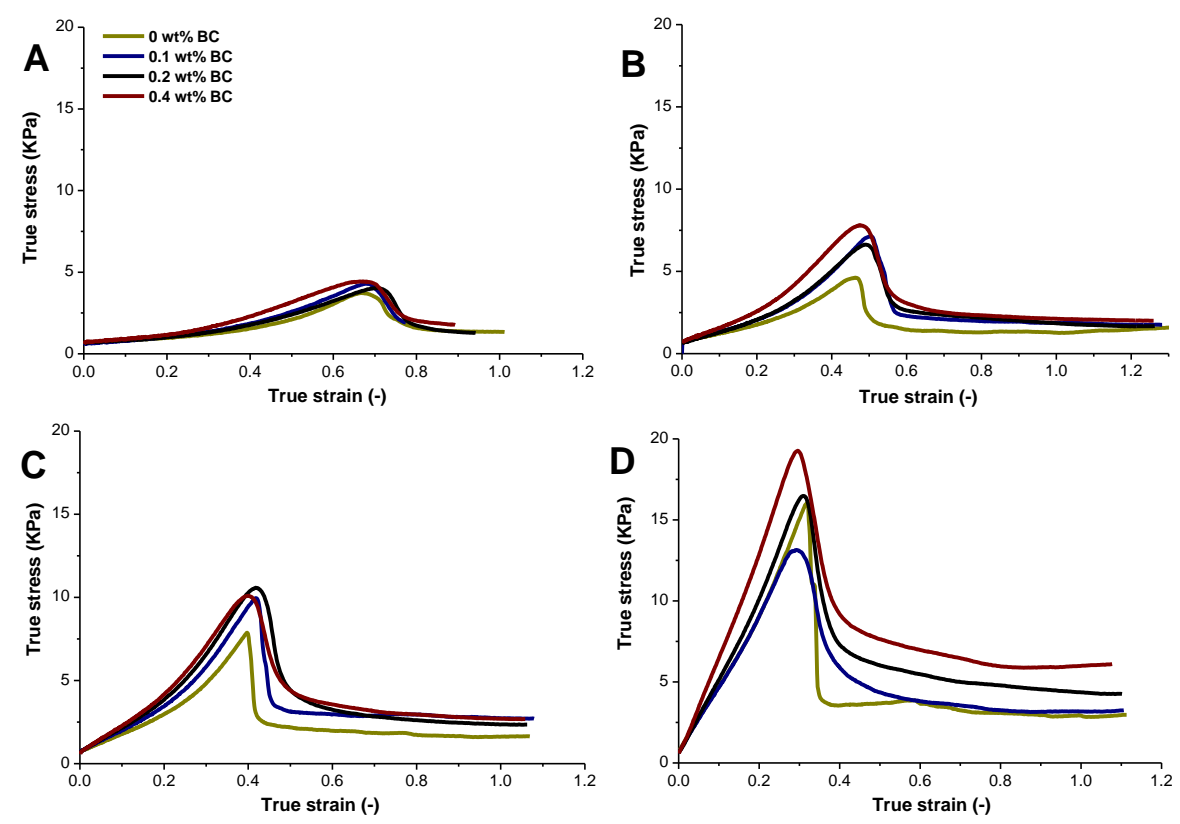

Figure 12 Uniaxial compression with $80 \%$ deformation of WPI-BC gels at pH 2 with $\mathrm{NaCl}$ concentrations of 0 (A), 50 (B), 100 (C), and 200 (D) mM. All samples contain a WPI concentration of $9 \mathrm{wt} \%$ and $\mathrm{BC}$ microfibril concentrations range between $0,0.1,0.2$, and $0.4 \mathrm{wt} \%$.

The Young's modulus reflects the stiffness of the gel. As shown in Figure 13A, increasing $\mathrm{NaCl}$ concentration increases the stiffness of the pure WPI gel. Upon addition of $\mathrm{BC}$ microfibrils, the gels become stiffer at all $\mathrm{NaCl}$ concentrations upon increasing $\mathrm{BC}$ microfibril concentration.

Overall, the large deformation results suggest that the concentration of $\mathrm{NaCl}$ only influences the WPI structure with according changes in rheological behaviour and fracture properties. 

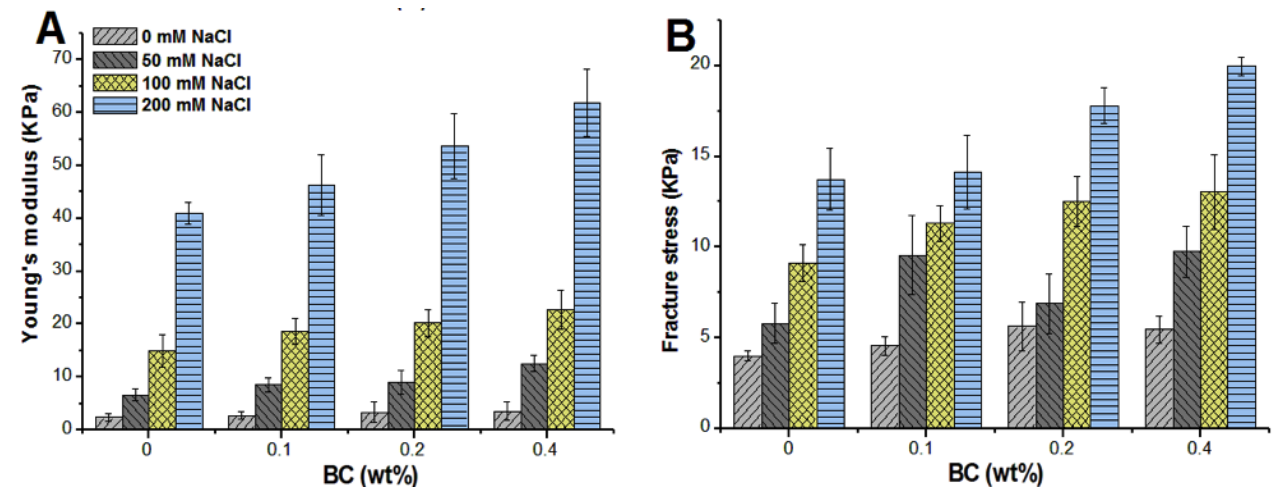

Figure 13 Young's modulus (A) and fracture stress (B) of WPI-BC gels as a function of BC microfibril concentration with $\mathrm{NaCl}$ concentrations of $0,50,100$, and $200 \mathrm{mM}$. All samples contain a WPI concentration of $9 \mathrm{wt} \%$ and $\mathrm{BC}$ concentrations range between $0,0.1,0.2$, and $0.4 \mathrm{wt} \%$. The error bar is based on the standard deviation of quadruplicate samples.

\subsection{Conclusion}

We have prepared bi-fibrillar gels from whey protein isolate and bacterial cellulose microfibrils at $\mathrm{pH} 2$ upon prolonged heating. In the presence of BC microfibrils, the WPI forms a fibrillar gel consisting of linear fibrillar aggregates. The two types of fibrillar structures co-exist and are overall homogeneous under the conditions studied. Increase of $\mathrm{BC}$ microfibrils increases the firmness of the gel. Upon increasing $\mathrm{NaCl}$ concentration in the bi-fibrillar gel, the WPI structure changes similarly as the WPI structure without the BC microfibrils present. The WPI fibrillar structure dominates the rheological properties of the bi-fibrillar gels. When fibrils from WPI are being formed near BC micofibrils, the BC microfibrils serve as a director for the WPI fibril formation. With the presence of $B C$ around the WPI, the fibrils formed are better aligned next to one another parallel to the $B C$ microfibrils.

\subsection{Acknowledgement}

This work is supported by NanoNextNL, a micro and nanotechnology consortium of the Government of the Netherlands and 130 partners. We thank Tiny Franssen-Verheijen for SEM image analysis. 


\subsection{References}

1. F. Chiti and C. M. Dobson, Annu. Rev. Biochem., 2006, 75, 333-366.

2. C. M. Dobson, Nature, 2003, 426, 884-890.

3. E. van der Linden, Food hydrocolloids, 2012, 26, 421-426.

4. U. Shimanovich, I. Efimov, T. O. Mason, P. Flagmeier, A. K. Buell, A. Gedanken, S. Linse, K. S. Åkerfeldt, C. M. Dobson and D. A. Weitz, ACS nano, 2015, 9, 43-51.

5. D. Oboroceanu, L. Wang, A. Kroes-Nijboer, A. Brodkorb, P. Venema, E. Magner and M. A. Auty, International Dairy Journal, 2011, 21, 823-830.

6. T. Nicolai, M. Britten and C. Schmitt, Food Hydrocolloids, 2011, 25, 1945-1962.

7. S. Loveday, X. Wang, M. Rao, S. Anema and H. Singh, J Agr Food Chem, 2011, 59, 8467-8474.

8. J.-M. Jung and R. Mezzenga, Langmuir, 2009, 26, 504-514.

9. A. C. Dave, S. M. Loveday, S. G. Anema, T. S. Loo, G. E. Norris, G. B. Jameson and H. Singh, J Agr Food Chem, 2013, 61, 7817-7828.

10. S. Bolisetty, L. Harnau, J.-m. Jung and R. Mezzenga, Biomacromolecules, 2012, 13, 3241-3252.

11. J. Adamcik and R. Mezzenga, Macromolecules, 2012, 45, 1137-1150.

12. C. Akkermans, P. Venema, A. J. van der Goot, H. Gruppen, E. J. Bakx, R. M. Boom and E. van der Linden, Biomacromolecules, 2008, 9, 1474-1479.

13. P. Aymard, T. Nicolai, D. Durand and A. Clark, Macromolecules, 1999, 32, 2542-2552.

14. L. N. Arnaudov and R. de Vries, Biomacromolecules, 2006, 7, 3490-3498.

15. A. Kroes-Nijboer, P. Venema and E. van der Linden, Food \& function, 2012, 3, 221-227.

16. J. De Wit, Journal of Dairy Science, 1998, 81, 597-608.

17. S. G. Bolder, H. Hendrickx, L. Sagis and E. Van der Linden, Applied Rheology, 2006, 16, 258-264.

18. S. G. Bolder, H. Hendrickx, L. M. C. Sagis and E. van der Linden, J Agr Food Chem, 2006, 54, 4229-4234.

19. S. G. Bolder, A. J. Vasbinder, L. M. C. Sagis and E. van der Linden, International Dairy Journal, 2007, 17, 846-853.

20. W. S. Gosal, A. H. Clark and S. B. Ross-Murphy, Biomacromolecules, 2004, 5, 2420-2429.

21. W. S. Gosal, A. H. Clark and S. B. Ross-Murphy, Biomacromolecules, 2004, 5, 2408-2419.

22. S. Ikeda and V. J. Morris, Biomacromolecules, 2002, 3, 382-389.

23. G. M. Kavanagh, A. H. Clark and S. B. Ross-Murphy, International Journal of Biological Macromolecules, 2000, 28, 41-50.

24. G. M. Kavanagh, A. H. Clark and S. B. Ross-Murphy, Langmuir, 2000, 16, 9584-9594.

25. M. Langton and A.-M. Hermansson, Food Hydrocolloids, 1992, 5, 523-539.

26. S. Loveday, X. Wang, M. Rao, S. Anema, L. Creamer and H. Singh, International Dairy Journal, 2010, 20, 571-579. 
27. S. M. Loveday, M. A. Rao, L. K. Creamer and H. Singh, Journal of Food Science, 2009, 74, R47R55.

28. L. M. C. Sagis, C. Veerman, R. Ganzevles, M. Ramaekers, S. G. Bolder and E. van der Linden, Food Hydrocolloids, 2002, 16, 207-213.

29. Z. Shi, Y. Zhang, G. O. Phillips and G. Yang, Food Hydrocolloids, 2014, 35, 539-545.

30. A. Okiyama, M. Motoki and S. Yamanaka, Food Hydrocolloids, 1992, 6, 479-487.

31. A. Okiyama, M. Motoki and S. Yamanaka, Food hydrocolloids, 1993, 6, 503-511.

32. H. Ougiya, K. Watanabe, Y. Morinaga and F. Yoshinaga, Bioscience, biotechnology, and biochemistry, 1997, 61, 1541-1545.

33. H. Zhu, S. Jia, H. Yang, W. Tang, Y. Jia and Z. Tan, Food Science and Biotechnology, 2010, 19, 1479-1484.

34. P. Paximada, E. Tsouko, N. Kopsahelis, A. A. Koutinas and I. Mandala, Food Hydrocolloids, 2016, 53, 225-232.

35. P. Paximada, A. A. Koutinas, E. Scholten and I. G. Mandala, Food Hydrocolloids, 2016, 54, 245254.

36. S. Cho and N. Almeida, Dietary fiber and health, CRC Press, 2012.

37. K. Lin and H. Lin, Journal of food science, 2004, 69, SNQ107-SNQ111.

38. S. B. LIN, L. C. CHEN and H. H. CHEN, Journal of Food Process Engineering, 2011, 34, 1363-1379.

39. R. Jonas and L. F. Farah, Polymer Degradation and Stability, 1998, 59, 101-106.

40. A. Kuijk, R. Koppert, P. Versluis, G. van Dalen, C. Remijn, J. Hazekamp, J. Nijsse and K. P. Velikov, Langmuir, 2013, 29, 14356-14360.

41. U. Geyer, T. Heinze, A. Stein, D. Klemm, S. Marsch, D. Schumann and H. P. Schmauder, International Journal of Biological Macromolecules, 1994, 16, 343-347.

42. C. Tokoh, K. J. Takabe and M. Fujita, Cellulose, 2002, 9, 65-74.

43. A. F. Jozala, L. C. de Lencastre-Novaes, A. M. Lopes, V. de Carvalho Santos-Ebinuma, P. G. Mazzola, A. Pessoa-Jr, D. Grotto, M. Gerenutti and M. V. Chaud, Applied microbiology and biotechnology, 2016, 1-10.

44. M. Phisalaphong and N. Chiaoprakobkij, Bacterial Cellulose: A Sophisticated Multifunctional Material, 2012, 9, 143-156.

45. S. J. Veen, A. Kuijk, P. Versluis, H. Husken and K. P. Velikov, Langmuir, 2014, 30, 13362-13368.

46. S. J. Veen, P. Versluis, A. Kuijk and K. P. Velikov, Soft matter, 2015, 11, 8907-8912.

47. V. Urbonaite, H. H. J. de Jongh, E. van der Linden and L. Pouvreau, J Agr Food Chem, 2014, 62, 7550-7558.

48. A. Clark, G. Kavanagh and S. Ross-Murphy, Food Hydrocolloids, 2001, 15, 383-400.

49. V. Bernal and P. Jelen, Journal of Dairy Science, 1985, 68, 2847-2852.

50. P. Relkin, L. Eynard and B. Launay, Thermochimica Acta, 1992, 204, 111-121.

51. S. Kasapis and I. M. Al-Marhoobi, Biomacromolecules, 2005, 6, 14-23. 
52. S. B. Ross-Murphy, Journal of Texture Studies, 1995, 26, 391-400.

53. G. Agoda-Tandjawa, S. Durand, C. Gaillard, C. Garnier and J. L. Doublier, Carbohyd Polym, 2012, 87, 1045-1057.

54. M. E. Bertrand and S. L. Turgeon, Food Hydrocolloids, 2007, 21, 159-166.

55. C. M. Bryant and D. J. McClements, Food Hydrocolloids, 2000, 14, 383-390.

56. S. M. Fitzsimons, D. M. Mulvihill and E. R. Morris, Food Hydrocolloids, 2008, 22, 576-586.

57. M. Beaulieu, S. L. Turgeon and J.-L. Doublier, International Dairy Journal, 2001, 11, 961-967.

58. D. V. Zasypkin, E. E. Braudo and V. B. Tolstoguzov, Food Hydrocolloids, 1997, 11, 159-170. 
Chapter 5 


\section{Chapter 6}

\section{Protein fibrils induce emulsion}

\section{stabilization}

Published as:

Jinfeng Peng, Joana Ralfas Simon, Paul Venema, Erik van der Linden. Protein fibrils induce emulsion stabilization. Langmuir, 2016, 32, 2164-2174. 


\section{Abstract}

The behavior of an oil-in-water emulsion was studied in the presence of protein fibrils for a wide range of fibril concentrations by using rheology, diffusing wave spectroscopy and confocal laser scanning microscopy. Results showed that above a minimum fibril concentration, depletion flocculation occurred, leading to oil droplet aggregation and enhanced creaming of the emulsion. Upon further increasing the concentration of the protein fibrils, the emulsions were stabilized. In this stable regime both aggregates of droplets and single droplets are present and these aggregates are smaller than the aggregates in the flocculated emulsion samples at the lower fibril concentrations. The size of the droplet aggregates in the stabilized emulsions is independent of fibril concentration. In addition, the droplet aggregation was reversible upon dilution both by a $\mathrm{pH} 2 \mathrm{HCl}$ solution and by a fibril solution at the same concentration. The viscosity of the emulsions containing fibrils was comparable to that of the pure fibril solution. Neither fibril networks nor droplet gel networks were observed in our study. The stabilization mechanism of emulsions containing long protein fibrils at high protein fibril concentrations points towards the mechanism of a kinetic stabilization. 


\subsection{Introduction}

Proteins have the ability to form various structures under different conditions. The morphologies of these structures range from linear to spherical. One of the linear structures is protein fibrils that can be formed using a wide range of proteins, such as, egg white proteins (ovalbumin ${ }^{1}$ and lysozyme ${ }^{2}$ ), soy protein, ${ }^{3}$ whey protein, ${ }^{4}$ and pea protein, ${ }^{5}$ when the appropriate conditions are used. For example, when heating $\beta$-Lactoglobulin ( $\beta$ $\mathrm{Ig}$ ) at low $\mathrm{pH}$ and low ionic strength, the protein is first hydrolyzed into small peptides and part of these peptides assemble into fibrils. ${ }^{6-8}$ These fibrils typically have a diameter in the order of nanometers, a length in the order of micrometers, ${ }^{9}$ and a persistence length around a micrometer. ${ }^{10,11}$ Their high aspect ratio and rod-like structure leads to a high excluded volume, ${ }^{12}$ making them interesting candidates as additives to colloidal dispersions.

The addition of non-adsorbing polymers to colloidal dispersions, can change the stability of the dispersion and has been frequently studied due to its fundamental aspects and wide range of applications in different fields. ${ }^{13}$ Above a minimum polymer concentration, polymers can induce depletion flocculation of the colloidal particles leading to phase separation. ${ }^{14,15}$ The flocculation rate increased with increasing polymer concentration, until reaching a critical concentration, above which the rate of flocculation decreased with increasing polymer concentration. At high enough polymer concentrations, the dispersion becomes stable. This phenomenon is known as depletion stabilization. ${ }^{16}$ Rods have been found to induce a rich phase behavior in colloidal dispersions. ${ }^{15,}{ }^{17-20}$ Blijdensteijn et al. studied the behavior of rod-like protein fibrils in emulsions. ${ }^{21}$ They suggested that the flocculation at low fibril concentrations was induced by depletion interaction. Here we investigate the stabilization mechanism of emulsions at high fibril concentrations, where the rod length and oil droplet diameter are comparable. Earlier studies on depletion interaction mainly focused on relatively low concentration of rods, and the size of rods being much smaller than that of the particles. ${ }^{15,}$ 22-28 We use rheology, diffusing wave spectroscopy and confocal laser scanning microscopy as experimental techniques. 


\subsection{Experimental section}

\subsubsection{Whey protein isolate (WPI) solution preparation}

WPI powder (Bipro, Davisco, Lot \# JE 198-1-420, USA) was dissolved in $\mathrm{HCl}$ solution of pH 2 to prepare a stock solution of $10 \mathrm{wt} \% \mathrm{WPI}$. The solution was left overnight in a cold room $\left(4{ }^{\circ} \mathrm{C}\right)$ while mildly stirring using a magnetic stirring bar. After dissolution, the $\mathrm{pH}$ of the protein solution was adjusted to $\mathrm{pH} 2$ with a $6 \mathrm{M} \mathrm{HCl}$ solution. Subsequently, the protein solution was filtered through a filter (Hydrophilic PES $0.45 \mu \mathrm{m}$, Millipore Millex-HP). The protein concentration of the stock solution was determined using a UV spectrophotometer (Cary 50 Bio, Varian) at a wavelength of $278 \mathrm{~nm}$, and a calibration curve determined from WPI-solutions at known concentrations. The WPI stock solution was stored at $4^{\circ} \mathrm{C}$ for further use in fibril formation and emulsion preparation.

\subsubsection{Fibril formation}

The WPI stock solution was diluted to a protein concentration of $4 \mathrm{wt} \%$ with a $\mathrm{HCl}$ solution at $\mathrm{pH}$ 2. The samples were placed in a heating block (RT 15 Power, IKA-WERKE) and stirred at $80^{\circ} \mathrm{C}$ for 22 hours to form fibrils. After this step the fibril solution was cooled down in ice-water and afterwards stored at $4^{\circ} \mathrm{C}$. The fibril concentration indicated throughout this paper refers to the protein concentration before heating.

We note that for the preparation of the emulsions with 3.25 wt \% fibrils we had to use a stock solution of fibrils prepared at $4.5 \mathrm{wt} \%$ of protein. This does not affect the average fibril length. ${ }^{4}$

\subsubsection{Oil-in-water (o/w) emulsion preparation}

WPI was used as an emulsifier to prepare a $40 \%(\mathrm{v} / \mathrm{v}) \mathrm{o} / \mathrm{w}$ emulsion. The emulsion was prepared in two steps. First, sunflower oil (Reddy, The Netherlands, obtained from a local supermarket) was mixed with a 1 wt \% WPI solution using a high speed blender ( Ultra Turrax, T25 digital , IKA), at a speed of 9000 RPM for 5 minutes. Subsequently, the emulsion was homogenized by 5 passes through a homogenizer (Labscope, Delta instruments, The Netherlands) at a pressure of 70 bar. The emulsion was stored at $4{ }^{\circ} \mathrm{C}$ before use and all experiments were performed within 12 hours after storage. 


\subsubsection{Sample preparation}

A range of concentrations of protein fibrils were prepared by diluting the 4 wt \% protein fibril solution and the emulsion was added to those fibril solutions. The final volume percentage of emulsion droplets in the mixture was $10 \%(\mathrm{v} / \mathrm{v})$. The concentration of protein fibrils in the mixture were: 0 (no fibrils added), 0.001, 0.005, 0.01, 0.05, 0.1, 0.5, 1, $1.5,2,2.25,2.5,2.75,3$ or $3.25 \mathrm{wt} \%$. The emulsion and protein fibril solution were mixed by mild agitation for $10 \mathrm{~s}$.

\subsubsection{Characterization of protein fibrils and emulsion}

\subsubsection{Length distribution of protein fibrils}

The length distribution of the fibrils was determined using flow-induced birefringence. The decay curves of the flow-induced birefringence after the cessation of flow were measured with a strain-controlled ARES rheometer (Rheometrics Scientific) equipped with a modified optical analysis module. ${ }^{29}$ From these decay curves the length distribution of the fibrils were determined. ${ }^{30}$

\subsubsection{Transmission electron microscopy (TEM)}

TEM was used to image the protein fibrils. After a 100 -fold dilution a droplet of the sample was placed on a carbon film supported by a copper grid. After $15 \mathrm{~s}$, the droplet was brought into contact with a filter paper to remove most of the water. Subsequently, a droplet of $2 \%$ uranyl acetate was added and removed after $15 \mathrm{~s}$ by another filter paper. Electron micrographs were taken using a transmission electron microscope (JOEL JEM1011, Tokyo, Japan).

\subsubsection{Size distribution of oil droplets}

The size distribution of oil droplets was measured using multi-angle static laser light scattering (Mastersizer MS2000, Malvern Instruments). The average diameter of the oil droplets, $D_{[3,2]}$, was around $1.9 \pm 0.2 \mu \mathrm{m}$ for all batches. 


\subsubsection{Characterization of emulsion stability}

\subsubsection{Visual observation}

Samples were placed at room temperature $\left(20^{\circ} \mathrm{C}\right)$ and images were taken directly after preparation ( 0 days) and after 1 day and 7 days.

\subsubsection{Creaming stability}

The emulsions were placed in glass tubes and monitored over time at $20{ }^{\circ} \mathrm{C}$. The backscattering intensity of laser light as a function of height was measured (Turbiscan, MA 2000). The intensity profile was measured at times 0,1 and 7 days. Interpretation of the data is based on an analysis described elsewhere. ${ }^{21,31}$

\subsubsection{Confocal laser scanning microscopy (CLSM)}

The microstructure of the cream layer of samples after 1 day and 7 days was imaged using CLSM (Zeiss 200 m Axiovert, Thornwood, NY, USA). Samples were placed in a chamber prepared from a microscope glass slide (Menzel-glaser, $50 \mathrm{~mm}$, Thermo Scientific, Braunschweig, Germany) and a cover slip (Menzel-Glaser, $15 \mathrm{~mm} \times 15 \mathrm{~mm}$, Thermo Scientific, Braunschweig, Germany). Images were made using an oil immersion objective (Plan-Apochromat, 100x/1.46 oil, Zeiss, Germany). One drop of 0.2 wt \% Nile blue (Merck, Germany) solution was added to the samples before imaging. Sample imaging was performed at an excitation wavelength of $488 \mathrm{~nm}$ and an emission wavelength of $525 \mathrm{~nm}$.

\subsubsection{Viscosity}

The viscosity of the samples was measured on a stress controlled rheometer (MCR302, Anton Paar), using a concentric cylinder geometry (CC17/T1/S-SN38492). A solvent trap was used to prevent evaporation. The samples were pre-sheared at a shear rate of $30 \mathrm{~s}^{-1}$. Subsequently, the viscosity was monitored by increasing the shear rate from 0.01 to $500 \mathrm{~s}^{-}$

${ }^{1}$. Measurements were performed at $20^{\circ} \mathrm{C}$.

\subsubsection{Strain sweep}

A strain sweep was performed on a stress controlled rheometer (MCR501, Anton Paar), using a concentric cylinder geometry (CC17/T1-SN3951). A solvent trap was used to 
prevent evaporation. The strain sweep was carried out at $20^{\circ} \mathrm{C}$, at frequency of $0.1 \mathrm{~Hz}$ varying strain from $0.01 \%$ to $100 \%$.

\subsubsection{Diffusing wave spectroscopy (DWS)}

In our study, DWS is used to monitor the diffusive motion of emulsion droplets at various fibril concentrations. Dispersions of only fibrils are completely transparent; therefore, DWS is a suitable technique to monitor the emulsion droplets.

The samples were analyzed directly after mixing using DWS in transmission mode. In DWS, the intensity autocorrelation function $g_{2}(t)$ of the multiple scattered light is measured. Following ref ${ }^{32} \mathrm{~g}_{2}(\mathrm{t})$ is related to the mobility of the emulsion droplets as

$$
g_{2}(t)-1=\beta\left(\int_{0}^{\infty} P(s) \exp \left(-1 / 3 k_{0}^{2}<\Delta r^{2}(t)>\frac{s}{l^{*}}\right) d s\right)^{2}
$$

where it follows from the Siegert relation that

$$
g_{2}(t)-1=\beta\left|g_{1}(t)\right|^{2}
$$

Here $\beta$ is a constant determined by the collection optics, $P(s)$ is the path length distribution function of photons in the sample, $k_{0}=2 \pi n / \lambda$, with $n$ the refractive index of the solvent and $\lambda$ the wavelength of the laser, $\left\langle\Delta r^{2}(t)\right\rangle$ is the mean square displacement of the droplets, and $\mathrm{I}^{*}$ is the photo transport mean free path.

The motion of the emulsion droplets, expressed by their mean square displacement (MSD) $\left\langle\Delta r^{2}(t)\right\rangle$ can be obtained by numerically inverting the correlation functions and using Equation 1. Since it is convenient to work in scaled variables we define $Q^{2}=\left(\frac{L k_{0}}{l^{*}}\right)^{2}<\Delta r^{2}(t)>$. L refers to the optical path length through the sample cell. In a purely viscous solution, the oil droplets would undergo Brownian motion (unhindered diffusive colloidal motion) and $Q^{2}$ would vary linearly with time $Q^{2} \sim D t$, where $D$ is the diffusion constant. In a viscoelastic medium the short time behavior of $Q^{2}$ can generally be described by a power law $\left\langle Q^{2}(t)>\sim D t^{p}\right.$ where $\mathrm{D}$ is the diffusion constant, $\mathrm{p}$ is a measure for the viscoelastic properties of the matrix. When $p \leq \frac{1}{2}$ the matrix behaves 
like a medium where $G^{\prime} \geq G^{\prime \prime}$, when $1 / 2<p<1$ the matrix behaves like a medium where $G^{\prime}$ $<G^{\prime \prime}$ and when $p=1$ the matrix is purely viscous. ${ }^{33}$

All the emulsion-fibril mixtures were monitored at $20^{\circ} \mathrm{C}$ immediately after mixing using DWS in transmission mode. A He-Ne laser $(20 \mathrm{~mW}, \lambda=633 \mathrm{~nm})$ was used. The width of the laser beam was expanded to $3 \mathrm{~mm}$ using a beam expander. The laser beam that passed the transparent glass cell with path length $L$ of $2 \mathrm{~mm}$ was collected by a single-mode optical fiber. Polarizers were used to assure that only the multiple scattered light was collected. The signal was split into two signals of equal intensity and directed into two photomultiplier tubes (ALV/SO-SIPD, Germany). The intensity auto-correlation function was measured using a multiple tau digital correlator (ALV-5000/60X0, Germany). Samples were analyzed for 2 minutes for each measurement. Results were averaged from 10 measurements.

\subsubsection{Effect of oil volume fraction on emulsion stability}

The emulsions were prepared to obtain droplet volume fractions of $0.1 \%, 0.25 \%, 0.5 \%$, $1 \%, 5 \%$ and $10 \%(\mathrm{v} / \mathrm{v})$ upon dilution of the stock $40 \%(\mathrm{v} / \mathrm{v})$ emulsion. Afterwards, the emulsions were mixed with fibril concentrations ranging from 0.001 wt\% to $3 \mathrm{wt} \%$. The stability was determined visually 7 days after preparation.

\subsubsection{Effect of fibril length on emulsion stability}

The fibrils were shortened using a high speed blender (Ultra Turrax, T25 digital, IKA) for 2 minutes at a speed of $12200 \mathrm{rpm}$. Fibrils were imaged using transmission electron microscopy to confirm that fibrils were shortened.

\subsubsection{Effect of temperature on emulsion stability}

The emulsions with a volume percentage of $10 \%(\mathrm{v} / \mathrm{v})$, and various fibril concentrations were kept at $45^{\circ} \mathrm{C}$ in an oven. Images were taken at 0,1 and 7 days after preparation. 


\subsection{Results}

\subsubsection{Characterization of protein fibrils}

The protein fibrils were characterized in terms of their length distribution and morphology using flow-induced birefringence measurements and TEM. Figure $1 \mathrm{a}$ and $\mathrm{b}$ show the TEM images of the protein fibrils taken from protein fibrils solution and shortened fibrils, both prepared at $4 \mathrm{wt} \%$. The length distribution of the protein fibrils formed from a $4 \mathrm{wt} \%$ whey protein isolate is also shown in Figure 1c. The shortened fibrils were too short to be measured using flow-induced birefringence.
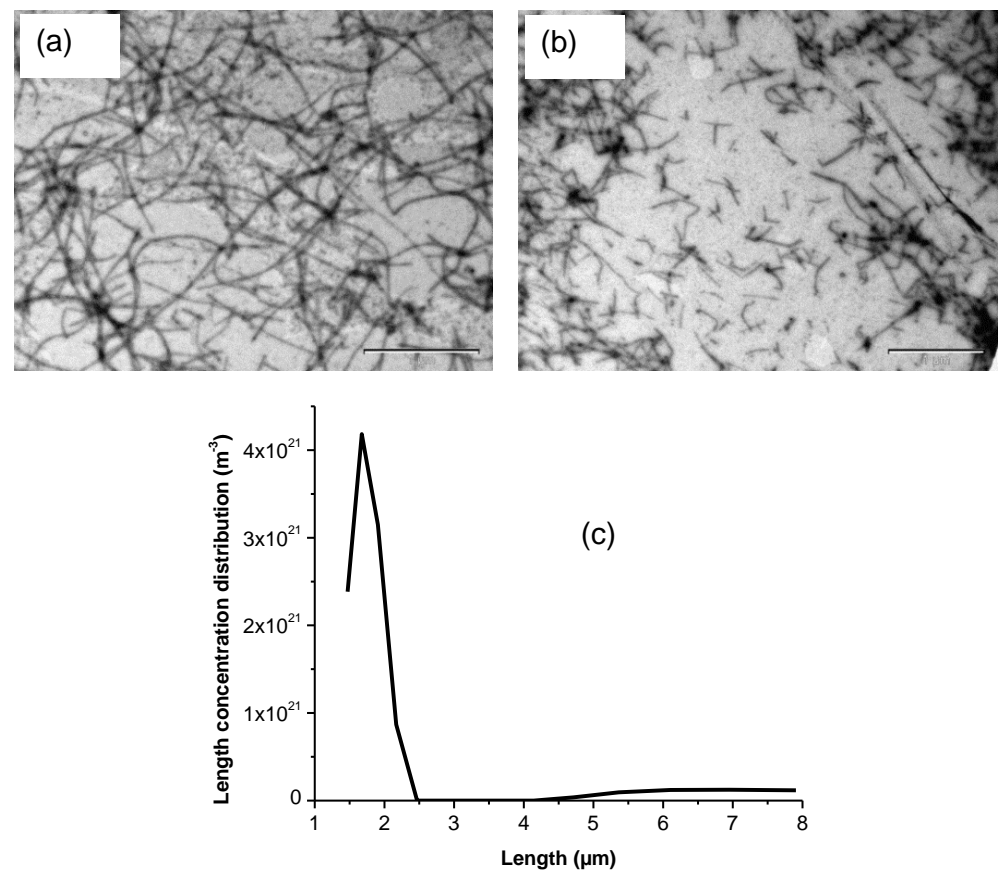

Figure 1 TEM images of (a) protein fibrils prepared by heating 4 wt \% WPI solution for 22 hours at $80^{\circ} \mathrm{C}$ and $\mathrm{pH} \mathrm{2,} \mathrm{(b)} \mathrm{shortened} \mathrm{fibrils} \mathrm{(scale} \mathrm{bars} \mathrm{correspond} \mathrm{to} 1 \mu \mathrm{m}$ ). (c) shows the length concentration distribution of the protein fibrils imaged in (a).

It was found that approximately $58 \mathrm{wt} \%$ of the protein is converted into fibrils under the conditions we used. ${ }^{34}$ The average length of the protein fibrils is around $2 \mu \mathrm{m}$, where the shortened ones have an average length of around $0.3 \mu \mathrm{m}$. Although the length 
concentration of fibrils will be the same, it can be clearly seen that fibrils have been shortened to a large extent (cf. Figure 1b). In order to determine the volume fraction of fibrils from their weight percentage, we use the size of its building blocks, i.e. peptides. ${ }^{7}$ We assume that one protein provides one peptide for fibril formation, and that the volume of one peptide equals $(\pi / 4) D^{2} l$, where $D=4 \mathrm{~nm}^{35}$ is used as the diameter and $\mathrm{I}=$ $2.4 \mathrm{~nm}$ as the length per peptide. ${ }^{7}$ The estimated volume fraction of fibrils at the corresponding fibril concentration used in our study can be found in Table A.1 (Appendix A).

\subsubsection{Emulsion stability in the presence of protein fibrils}

The stability of emulsions upon addition of protein fibrils was investigated. Figure 2a shows images of samples at $20^{\circ} \mathrm{C}$ at 0 days, 1 and 7 days after preparation, and in Figure $2 b$ - e the corresponding backscattering intensity profiles are shown. For ease of discussion, we discriminate four types ( $A$ to $D$ ) of creaming behavior, depending on fibril concentrations. 
(a)
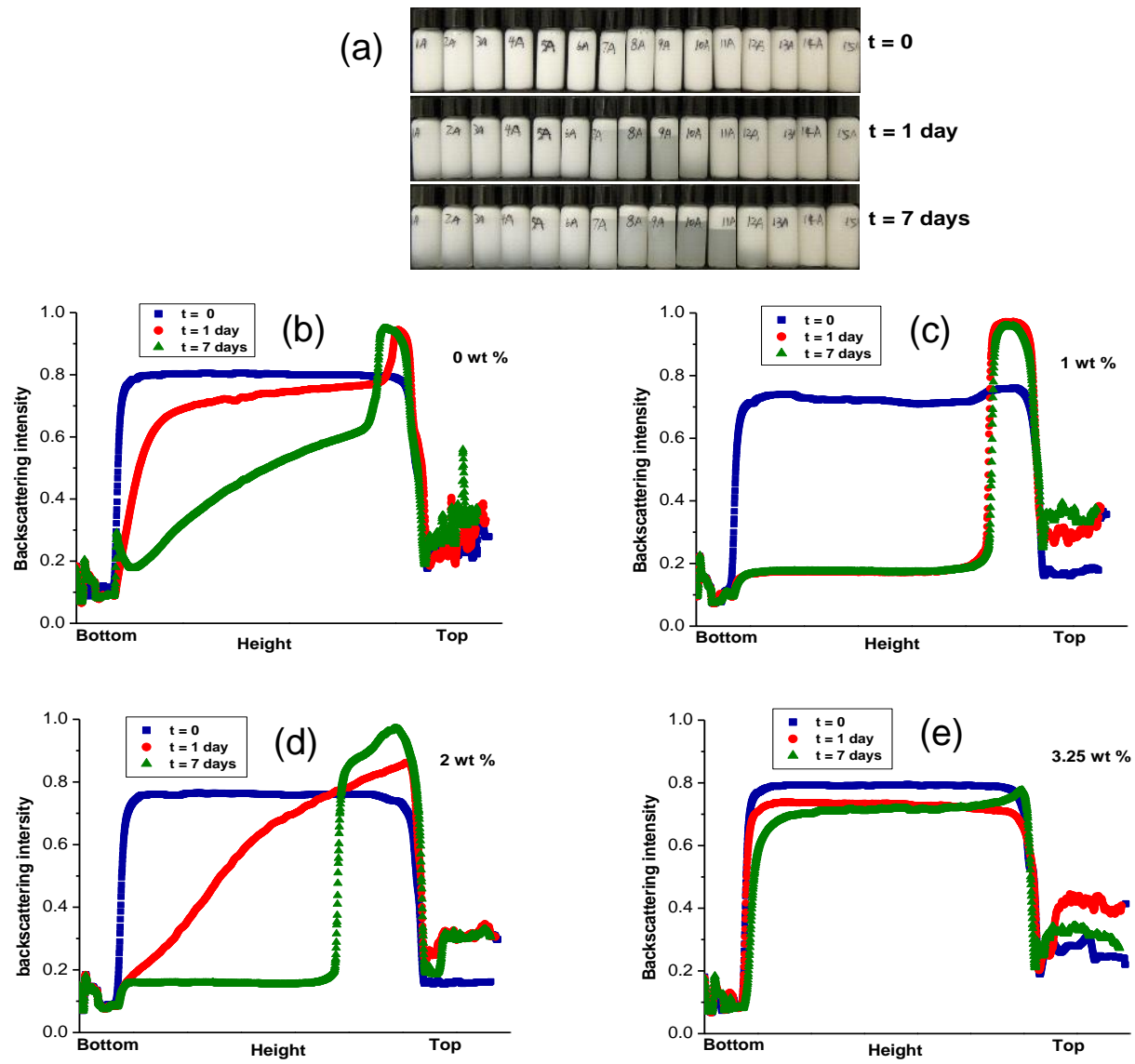

Figure 2 (a): Images of samples containing $10 \%(v / v)$ o/w emulsions and $0,0.001,0.005,0.01$, $0.05,0.1,0.5,1,1.5,2,2.25,2.5,2.75,3$ or 3.25 wt \% protein fibrils (from left to right in the image); (b), (c), (d) and (e): backscattering intensity profiles of samples containing $10 \%(\mathrm{v} / \mathrm{v}) \mathrm{o} / \mathrm{w}$ emulsion and $0 \mathrm{wt} \%$ (b), $1 \mathrm{wt} \%$ (c), 2 wt \% (d) and $3.25 \mathrm{wt} \%$ (e) protein fibrils. The horizontal axis represents the position along the tube.

Type A. The typical behavior of emulsions containing protein fibrils between $0-0.1$ wt \%, is shown in Figure $2 b$ (only 0 wt \% fibrils is shown). The samples gradually form a cream layer, indicating that adding low concentrations of protein fibrils does not stabilize the emulsion.

Type B. The typical behavior of emulsions containing protein fibrils between 0.5 wt $\%-1.5$ wt \%, is shown in Figure 2c (only 1 wt \% fibrils is shown). Already after 1 day the emulsions 
show a cream layer with a sharp boundary, as indicated by the backscattering intensity profile. Over time the profile for all these samples reflects typical creaming behavior caused by a density difference.

Type C. The typical behavior of emulsions containing protein fibrils between 2 wt $\%-2.25$ wt $\%$, is shown in Figure $2 \mathrm{~d}$ (only 2 wt \% fibrils is shown). After 1 day a diffuse creaming zone is observed, which develops into a sharp boundary, after 7 days.

Type D. The typical behavior of emulsions containing protein fibrils between $2.5 \mathrm{wt} \%$ 3.25 wt \%, is shown in Figure 2e (only 3.25 wt \% fibrils is shown). At these concentrations the emulsions were stabilized, and no creaming could be observed within 7 days. We note that these emulsions are more stable than the ones of type $A$.

To investigate the microstructure of the emulsions, the top layers of the emulsions after 1 day and 7 days of preparation was analyzed using confocal laser scanning microscopy. Figure 3 shows the images obtained by CLSM. 

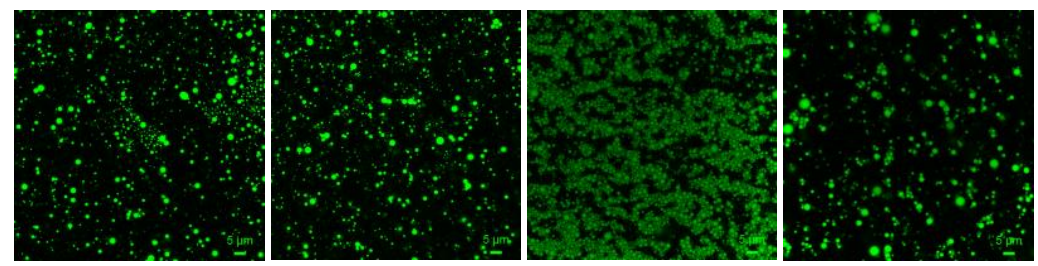

(a) $t$ = 1 day

0 wt \%

$$
0.01 \text { wt } \%
$$

1.5 wt \%

3 wt \%

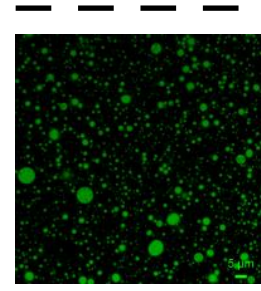

0 wt \%

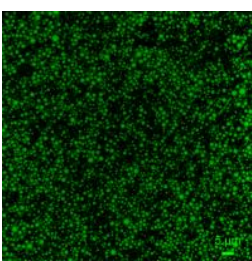

1 wt $\%$

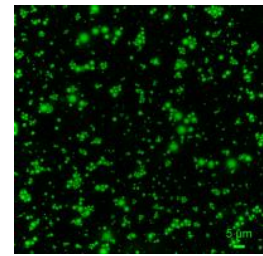

2.75 wt \%

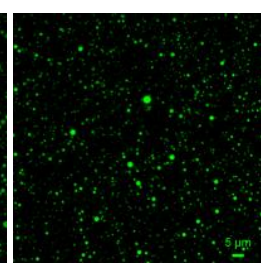

0.01 wt \%

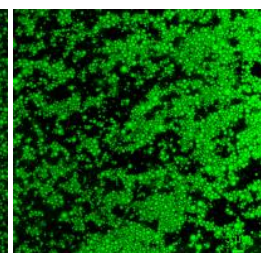

1.5 wt $\%$

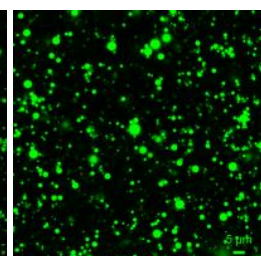

3 wt \%

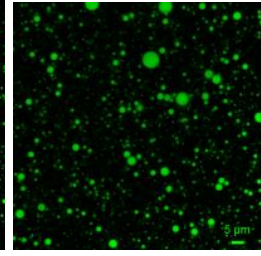

0.05 wt $\%$

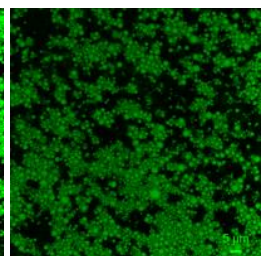

2 wt \%

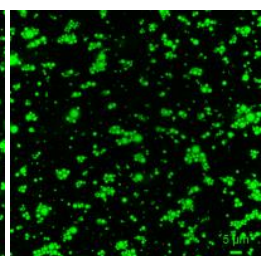

3.25 wt \%

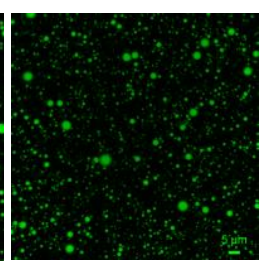

0.1 wt $\%$

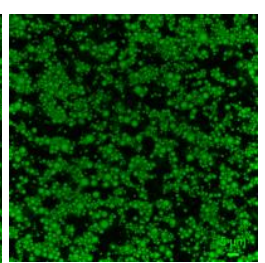

2.25 wt \%

2.5 wt \%

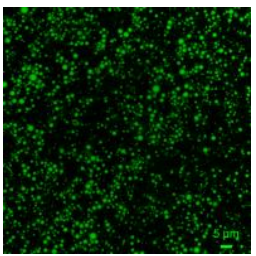

0.5 wt $\%$

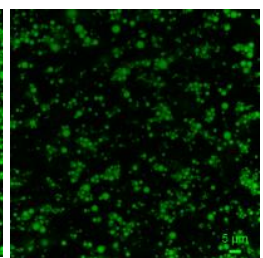

(b) $t=7$ days

Figure 3 CLSM images of the top layers of emulsions at 1 day (a) and 7 days (b) after preparation. The scale bar corresponds to $5 \mu \mathrm{m}$. The emulsions contain $10 \%$ oil and a fibril concentration of (a): $0,0.01,1.5$, or 3 wt \%; (b): 0, 0.01, 0.05, 0.1, 0.5 wt \% (top row), 1, 1.5, 2, 2.25, 2.5 wt \% (middle row), 2.75, 3 or 3.25 wt \% fibrils (bottom row), from left to right. Fibril concentrations are indicated below each image. The dotted line is used to differentiate samples imaged 1 day (a) and 7 days (b) after preparation.

From Figure $3 b$, the oil droplets on the top layer of emulsions containing fibrils from 0.001 wt $\%$ and $0.1 \mathrm{wt} \%$ were homogeneously distributed, similar to the emulsion without addition of fibrils ( 0 wt \% fibrils). Increasing fibril concentration from 0.5 wt \% to 2.25 wt \%, aggregation of droplets can be observed from CLSM. The extent of droplet 
aggregation enhanced with increasing fibril concentration. Droplets reached the largest extent of aggregation in the emulsion containing $1.5 \mathrm{wt} \%$ fibrils. The extent of aggregation decreased gradually for fibril concentrations between $2 \mathrm{wt} \%$ to $2.25 \mathrm{wt} \%$. At fibril concentrations higher than 2.5 wt \%, the aggregates were substantially smaller. However, from the backscattering and visual observation, it was found that these samples were stable for 7 days after preparation.

The microscopic images of the emulsions show that, at relatively low fibril concentration, the emulsions showed creaming rates comparable to that of the emulsion without fibrils and no flocculation of oil droplets could be observed. Emulsions with higher fibril concentrations did show enhanced creaming rates and extensive flocculation of oil droplets. Blijdenstein et al. ${ }^{21}$ focused on this enhanced creaming, which they attributed to depletion interactions ${ }^{20}$ induced by protein fibrils.

Emulsions at even higher fibril concentrations, remarkably, turned out to be stable, i.e. no creaming could be observed. This is in contrast to the emulsion without fibrils where still some creaming could be observed. At these higher fibril concentrations the oil droplets were present in aggregates consisting of a few droplets. It was observed that the typical size of these aggregates ( $~ 5 \mu \mathrm{m}$ ) seemed to be independent of fibril concentration above a critical fibril concentration (i.e. 2.75 wt \%). In addition, no droplet network could be observed, excluding the possibility of an emulsion gel.

In comparison to CLSM images of emulsions with fibrils after 1 day of preparation in Figure 3a, we did not observe significant changes of the droplet structures 7 days after preparation, neither in flocculated emulsions nor in stable emulsions at high fibril concentrations (cf. Figure $3 \mathrm{~b}$ ). This indicates that the droplet aggregates in the stable emulsions at higher fibril concentrations did not change with time.

\subsubsection{Rheological properties}

The shear viscosity of emulsions with protein fibrils was measured and compared to that of the protein fibril solutions (Figure $4 a$ and b). The result of strain sweep is shown in Figure 4c. 

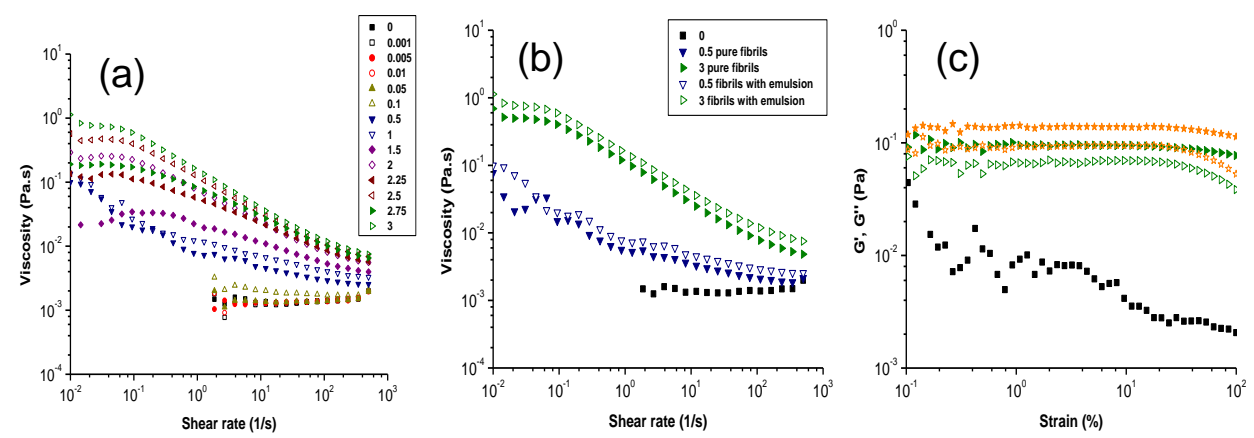

Figure 4 (a): Flow curves of $10 \%(v / v)$ emulsions containing protein fibrils with concentrations of $0,0.001,0.005,0.01,0.05,0.1,0.5,1,1.5,2,2.25,2.5,2.75$ or $3 \mathrm{wt} \%$, (b): flow curves of $0.5 \mathrm{wt} \%$ and $3 \mathrm{wt} \%$ protein fibril solutions and of $10 \%(\mathrm{v} / \mathrm{v})$ emulsions with $0.5 \mathrm{wt} \%$ and $3 \mathrm{wt} \%$ fibrils added. (c): The storage modulus, G' (open symbols), and loss modulus, G"(filled symbols), as a function of strain for $10 \%(\mathrm{v} / \mathrm{v}$ ) emulsions containing 0 (squares), 2.75 (triangles) and 3.25 (stars) wt \% protein fibrils, respectively. The storage modulus of the $10 \%(\mathrm{v} / \mathrm{v})$ emulsion without fibrils was too low to be measured.

The flow curves of a $10 \%(v / v)$ emulsion without fibrils and with fibrils below fibril concentration of $0.1 \mathrm{wt} \%$ show Newtonian behavior (Figure 4a). At fibril concentrations above $0.1 \mathrm{wt} \%$, the emulsions show shear thinning behavior. The viscosity increases upon increasing fibril concentration. Figure $4 \mathrm{~b}$ shows that the solutions of fibrils show shear thinning behavior. In addition, the viscosity of emulsions containing fibrils did not show significant increase compared to pure fibril solutions at the same fibril concentration (cf. Figure $4 b$ ), therefore, it can be concluded that the viscosity of the emulsions containing fibrils is mainly caused by the presence of fibrils.

All emulsions containing fibrils show a loss modulus ( $\left.G^{\prime \prime}\right)$ higher than the storage modulus $\left(G^{\prime}\right)$, indicating a liquid like structure (cf. Figure $4 c$ ) where no gel is formed.

\subsubsection{Diffusing wave spectroscopy}

The intensity autocorrelation function obtained is shown in Figure 5a. As a measure for the diffusion coefficient of the oil droplets, the half-life time of the decorrelation, $\tau_{1 / 2}$, was determined by $g_{2}\left(\tau_{1 / 2}\right)-1=1 / 2{ }^{36}$ The calculated $Q^{2}$, half-life time and the $p$ value are also shown in Figure $5 b, c$ and $d$. 

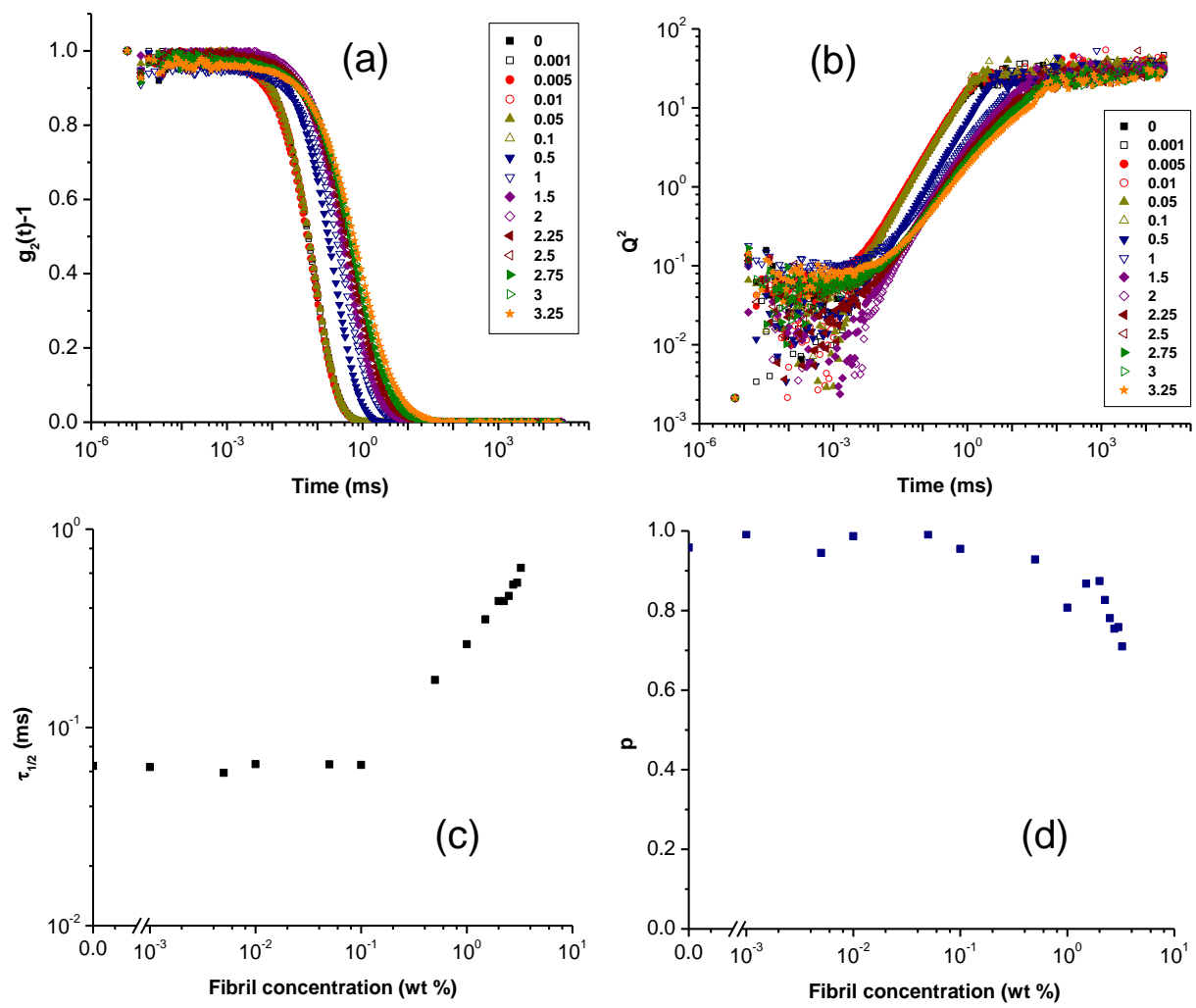

Figure 5 The $g_{2}(t)-1(a), Q^{2}(b), \tau_{1 / 2}(c)$ and $p$ value (d) of samples containing $10 \%(v / v)$ emulsion and $0,0.001,0.005,0.01,0.05,0.1,0.5,1,1.5,2,2.25,2.5,2.75,3$, or 3.25 wt \% fibrils recorded immediately after sample preparation.

The mobility of oil droplets is reflected by the intensity autocorrelation function and the calculated MSD. Adding small amounts of fibrils ( $\leq 0.1 \mathrm{wt} \%$ ) did not significantly change the position and shape of the curves, i.e., similar to the emulsion without fibrils. The increase of protein fibril concentration ( $\geq 0.5 \mathrm{wt} \%$ ) resulted in a shift of the curves towards longer decorrelation times, without significantly changing the shape of the curve (cf. Figure $5 \mathrm{a}$ and b). This implies a decrease in mobility of the emulsion droplets, due to a viscosity increase of the continuous phase and/or droplet aggregation. ${ }^{37}$ 
Figure $5 \mathrm{c}$ shows that a small amount of fibrils ( $\leq 0.1 \mathrm{wt} \%)$ did not significantly change the half-life time. However, above 0.5 wt \% of fibrils, the half-life time increased. It is interesting to compare the half-life time for flocculated and stabilized emulsions. At 2.25 wt \% fibrils where the emulsion is flocculated we have $\tau_{1 / 2}=0.4 \mathrm{~ms}$ while at $2.5 \mathrm{wt} \%$, where the emulsion is stable, we have $\tau_{1 / 2}=0.6 \mathrm{~ms}$. The half-life time, $\tau_{1 / 2}$, is inversely proportional to the droplet diffusion coefficient, and the diffusion coefficient, $D$, is inversely proportional to the viscosity, $\eta$ according to $D=\frac{k_{B} T}{6 \pi \eta a}$. Therefore the ratio of viscosities between the flocculated ( $2.25 \mathrm{wt} \%$ ) and stabilized ( $2.5 \mathrm{wt} \%$ ) emulsions is only a factor of $2 / 3$. This would only slow down the flocculation by a factor of $2 / 3$, and not explain the stabilization by an increased viscosity only. From fitting of the $Q^{2}$ curve, Figure $5 \mathrm{~b}$, and using $<Q^{2}(t)>\sim D t^{p}$ we obtain $\mathrm{p}>0.7$ (Figure $5 \mathrm{~d}$ ), meaning that $\mathrm{G}^{\prime}<\mathrm{G}^{\prime \prime},{ }^{33}$ which is in line with the rheological measurements, where no gel formation could be observed. ${ }^{37}$ However, with increasing the fibril concentration, we did observe a decrease of $p$ value, which indicated that the emulsions have viscoelastic properties.

\subsubsection{Effect of oil volume fraction on emulsion stability}

Images of emulsions with different oil volume fractions and fibril concentrations are shown in Figure 6a. Additionally, the stability diagrams of emulsions after 7 days are shown in Figure 6b. 
(a) $0.1 \% \mathrm{v} / \mathrm{v}$

(b) $0.25 \% \mathrm{v} / \mathrm{v}$

(c) $0.5 \% \mathrm{v} / \mathrm{v}$
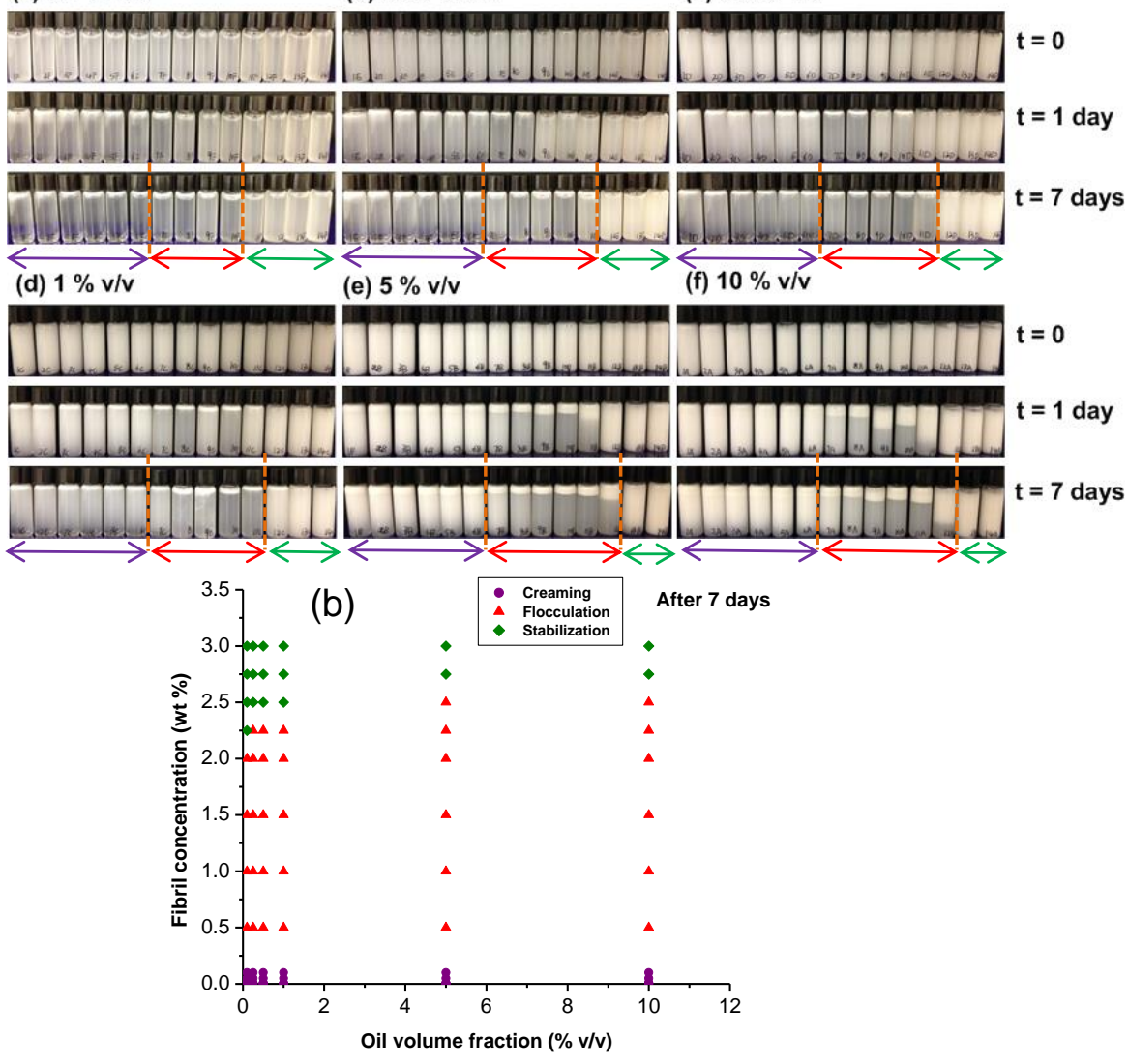

Figure 6 (a): Visual appearance at $20^{\circ} \mathrm{C}$ of emulsions containing oil volume fraction of (a) $0.1 \%$, (b) $0.25 \%$, (c) $0.5 \%$, (d) $1 \%$, (e) $5 \%$, or (f) $10 \%$ (v/v) with fibril concentration of $0,0.001,0.005,0.01$, $0.05,0.1,0.5,1,1.5,2,2.25,2.5,2.75$ or $3 \mathrm{wt} \%$ (from left to right). The rows correspond to the age of the samples after preparation. The lines are used to differentiate the 3 stability regimes. (b): the corresponding stability diagram of emulsions with different volume fractions containing different fibril concentrations at $\mathbf{7}$ days after preparation. The three different stability regimes were described as creaming (spheres), flocculation (triangle) and stabilization (squares).

Emulsions containing different oil volume fractions (Figure $6 a$ and b) show similar regions where creaming, flocculation and stabilization, occurs. At concentration of fibrils below 0.5 wt \%, fibrils do not change the creaming compared to the sample without fibrils. Above 0.5 wt \% fibrils, droplet flocculation (as confirmed by CLSM) leading to enhanced creaming was observed. At high enough fibril concentration, the emulsions are stable. For 
all volume fractions of emulsions, flocculation occurs at fibril concentrations above 0.5 wt \%. The main difference between the different emulsions is the fibril concentration at which the emulsion becomes stable. With increasing oil volume fraction, the fibril concentration needed to stabilize the emulsion slightly increases.

\subsubsection{Effect of fibril length on emulsion stability}

Protein fibrils were shortened to an average length about $0.3 \mu \mathrm{m}$ and mixed with a $10 \%$ $(v / v)$ emulsion. The stability of these emulsions was compared to that of $10 \%(v / v)$ emulsions containing fibrils with an average length of about $2 \mu \mathrm{m}$ (cf. Figure 2a). Figure 7a shows images of the emulsions containing the shortened protein fibrils (cf. Figure 2a for the stability of emulsions containing the longer (i.e. $2 \mu \mathrm{m}$ ) fibrils). Figure $7 \mathrm{~b}$ shows the stability diagram of emulsions as a function of different average fibril lengths and fibril concentrations.

(a)

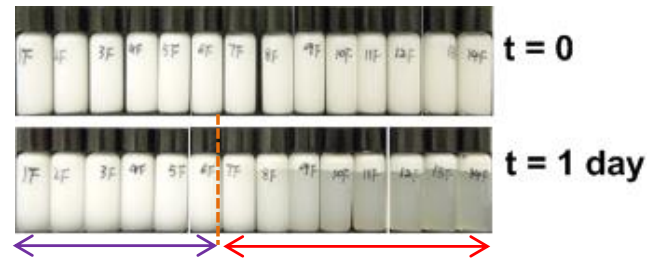

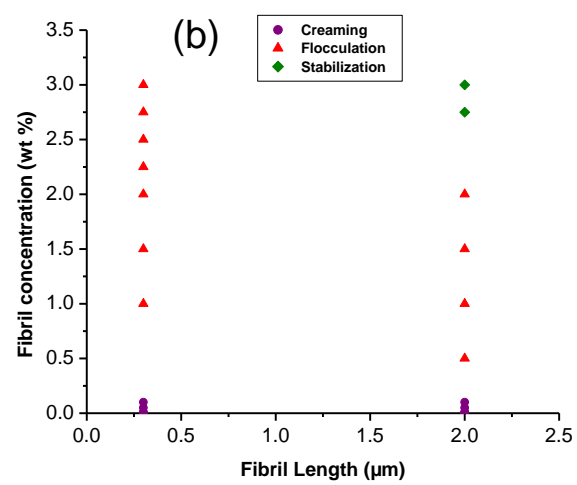

Figure 7 (a): Images of emulsions containing $10 \%$ (v/v) oil droplets with 0 (i.e. no fibrils added), $\mathbf{0} .001,0.005,0.01,0.05,0.1,0.5,1,1.5,2,2.25,2.5,2.75$ and 3 wt \% protein fibrils added, with an average length of $0.3 \mu \mathrm{m}$. The concentration of fibrils increases from left to right. The lines are used to differentiate the $\mathbf{2}$ stability regimes. (b): stability diagram of emulsions with fibrils at different concentrations and lengths. The three different stability regimes were described as creaming (spheres), flocculation (triangle) and stabilization (squares).

The shortened fibrils did not stabilize the emulsion, up to fibril concentration of $3 \mathrm{wt} \%$ (cf. Figure 7a). This result is to be compared to Figure $2 a$, in which emulsions with longer fibrils at and above $2.5 \mathrm{wt} \%$ remained stable even after 7 days of preparation. However, 
the emulsions with the shortened fibrils did induce depletion flocculation, above 1 wt \% of fibrils.

The onset of flocculation started for the short fibrils at a concentration of 1 wt \%, while for the longer fibrils, the onset occurs at a lower fibril concentration of 0.5 wt \%. This indicates that shorter fibrils are less efficient in depletion flocculation, in line with depletion theory that longer fibrils has a larger excluded volume ${ }^{20}$ and also earlier experiments by Blijdenstein et al.. ${ }^{21}$ The fact that the longer fibrils but not the shortened fibrils are able to stabilize emulsions above a certain fibril concentration also suggests that longer fibrils are more efficient in stabilizing emulsions.

\subsubsection{Effect of temperature on emulsion stability}

The stability of emulsions stored at $45{ }^{\circ} \mathrm{C}$ was compared to the stability of emulsions stored at $20{ }^{\circ} \mathrm{C}$ (cf. Figure 2a). Figure $8 \mathrm{a}$ shows the images of emulsions, containing various concentrations of fibrils, kept for 7 days at $45{ }^{\circ} \mathrm{C}$. Figure $8 \mathrm{~b}$ shows the stability diagram for the two temperatures. The stability diagram (cf. Figure $8 \mathrm{~b}$ ) shows that the fibril induced stabilization is temperature independent.

(a)

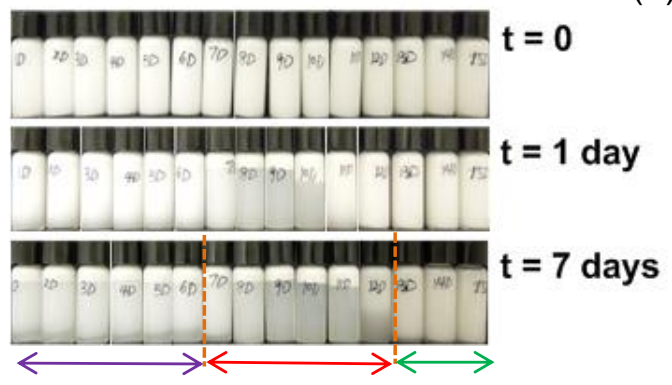

(b)

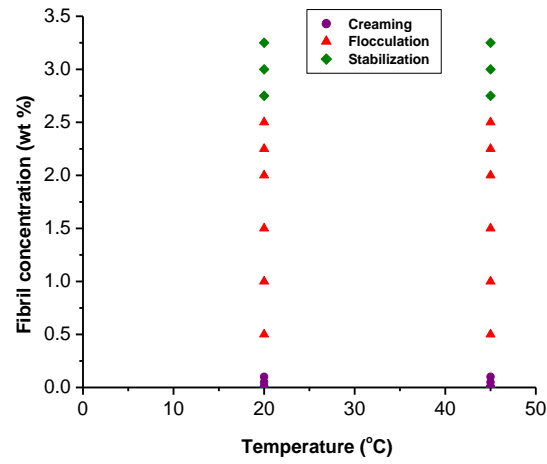

Figure 8 (a): Images of samples at $45{ }^{\circ} \mathrm{C}$ of $10 \%$ (v/v) emulsions with 0 (no fibrils added), 0.001 , $0.005,0.01,0.05,0.1,0.5,1,1.5,2,2.25,2.5,2.75,3$ or 3.25 wt \% protein fibrils with an average length of $2 \mu \mathrm{m}$. The concentration of fibrils increases from left to right. The lines are used to differentiate the 3 stability regimes. (b) Stability diagram of emulsions with different fibril concentrations and temperatures. The three different stability regimes were described as creaming (spheres), flocculation (triangle) and stabilization (squares). 


\subsubsection{Reversibility of aggregation}

To check whether the oil droplet aggregation that occurs at different fibril concentrations is reversible, dilution tests were performed. After 7 days of sample preparation, the top layer of the samples was diluted in two ways. One is by adding a pH 2 solution to reach a final fibril concentration of $0.1 \mathrm{wt} \%$. The other is by diluting the emulsion ten-fold, keeping the concentration of fibrils constant. Independent of the dilution method used, all emulsions could be re-dispersed upon dilution. To assure homogeneous mixing, samples were gently shaken directly after dilution. Two days after diluting the emulsions (i.e. 9 days after preparation) their microstructure was imaged using CLSM. The microstructures were compared with those of the undiluted emulsion (i.e. also 9 days old, cf. Figure 9).

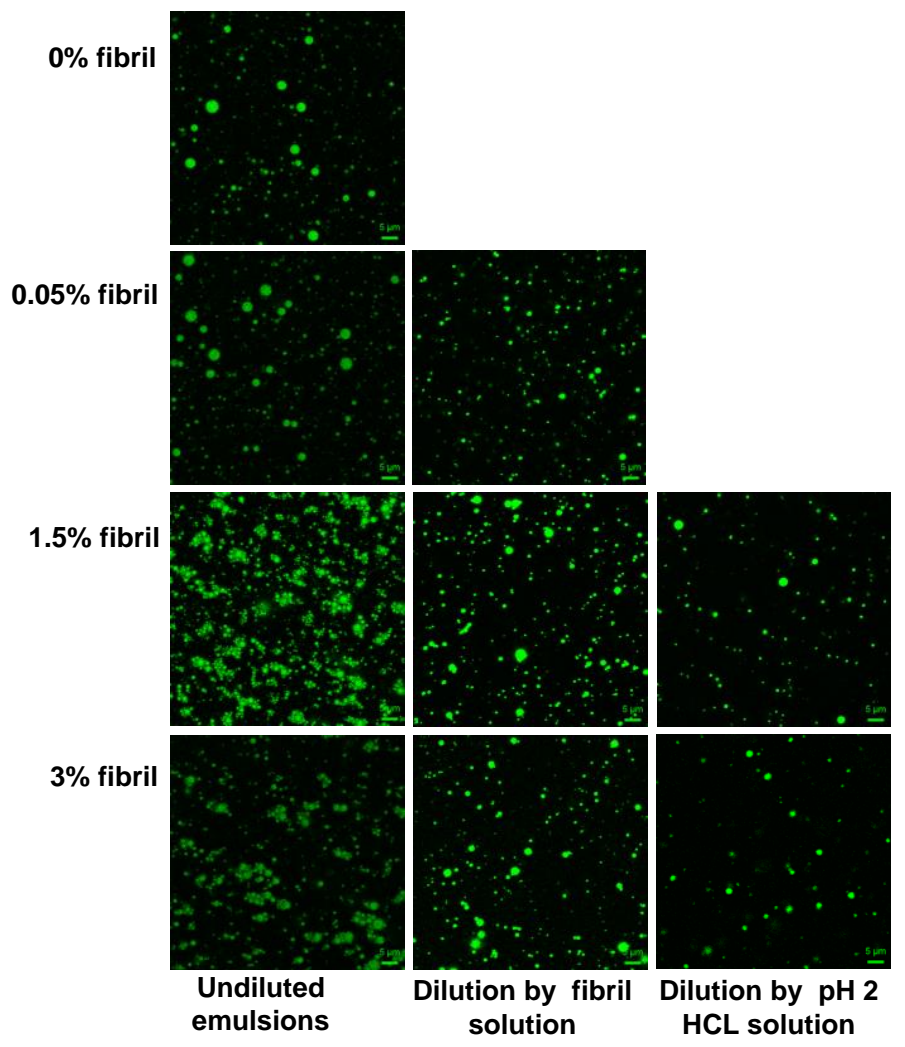

Figure 9 CLSM images of samples taken from the top layers of emulsions (all 9 days old). After 7 days of sample preparation the top layer of the samples was diluted in two ways. One is by adding a pH 2 solution to reach a final fibril concentration of $0.1 \mathrm{wt} \%$. The other is by diluting the 
emulsion ten-fold, keeping the concentration of fibrils constant. The first column depicts the microstructure of the initial emulsion top layer after 9 days. The fibril concentration refers to the fibril concentration in the initial emulsion. The scale bar corresponds to $5 \mu \mathrm{m}$.

The aggregates turn out to decrease in size upon dilution for both methods of dilution, towards the size of single droplets. This implies the droplet aggregation is a reversible process with the binding energy between droplets in the order of thermal energy, $\mathrm{k}_{B} T$.

\subsection{Discussion}

The addition of protein fibrils to emulsions leads to a complex behavior depending on the fibril concentration. As a function of fibril concentration the emulsions first show creaming at a similar rate as the emulsion without fibrils. Further increasing the fibril concentration leads to enhanced creaming, as observed earlier by Blijdensteijn et al. ${ }^{21}$, which was suggested to originate from depletion interaction. The temperature independency of the enhanced creaming is in line with the entropy driven nature of the depletion interaction. The dependency of average fibril length on the fibril concentration that is needed to induce flocculation is also in line with a depletion effect. The low concentration of fibrils needed to induce the depletion flocculation (volume fraction of fibrils is $\sim 0.001$ ) show that fibrils are very efficient depletion agents ${ }^{15}$. Above a certain fibril concentration the emulsions are completely stable against creaming.

Our microscopic observations show that the enhanced creaming is concomitant with aggregates of oil droplets. With increasing fibril concentration, we first observe larger and denser aggregates of oil droplets. Upon further increasing the fibril concentration, we observe a decrease in aggregate size. The aggregate size becomes independent of fibril concentration above a specific concentration. At and above this specific concentration the emulsion is stable, with aggregates and also single droplets present together. Observing the aggregates under a microscope over a time period of minutes does not show any change in size. When we dilute (ten-fold) the top part of these stable emulsions, containing aggregates and single droplets, with a fibril solution of the same fibril concentration, we observe, a decrease in aggregate size (observation made after 2 days of storage). The same holds for diluting the emulsion by the solvent ( $\mathrm{pH} 2$ solution) until a fibril concentration of $0.1 \mathrm{wt} \%$. We note that during the dilution experiment the samples are mildly agitated in order to ensure a homogeneous dilution procedure. This implies that 
the disaggregation upon dilution does not have to occur spontaneously. However, we can conclude that the interaction energy between the droplets is probably in the order of a few $k_{B} T$, since the aggregates break up under mild agitation.

A possible cause for the stability was previously suggested to be high viscosity of the continuous phase. ${ }^{21}$ However, this would only delay the creaming and not completely stop it, as is found in our experiments. An additional reason why this suggestion is less likely is that our DWS measurements reveal only a minor decrease in diffusivity of the oil droplets, and therefore only a minor increase in viscosity of the continuous phase. Our rheological measurements and DWS measurements also rule out the gelation of the continuous phase or the formation of a network of oil droplets.

The stabilization of colloidal dispersions in the presence of a non-adsorbing polymer or spherical particle at higher polymer concentrations has been observed and studied experimentally. ${ }^{38-44}$ In particular, the formation of particle aggregates or clusters has been observed in these stable colloidal dispersions. In our study, we observed both single particles and droplet aggregate present together in the stable emulsions at higher fibril concentrations. The mechanism of the stabilization has been argued to be either thermodynamic $^{42-51}$ or kinetic. ${ }^{16,22-24,39,41,52-54}$ Some also argued to be the slowdown of particle movement due to high concentration of polymer. ${ }^{40,55}$

Different theories have been put forward regarding thermodynamic and kinetic points of view. We like to mention the work of Feigin and Napper ${ }^{16}$ who attribute dispersion stability to a repulsive energy barrier, i.e. a kinetic effect. In contrast, Vincent et al., ${ }^{44,45,56}$ and later Fleer and co-workers, ${ }^{47-49}$ proposed a thermodynamic picture, with stabilization being caused by suppression of the depletion layer at higher concentrations of the depleting polymer. A different mechanism for the suppression of a depletion layer was put forward by Gast and Leibler. ${ }^{50,51}$ Another thermodynamic picture is given by Dutta and Green $^{57}$ who attribute stability to a vanishing surface tension between the particle and its surrounding. Yet another thermodynamic model is suggested by Groenewold and Kegel ${ }^{58}$ who calculated the stability regime of aggregates as a function of their size and shape by taking into account a short range attraction and a long range, electrostatic repulsion.

All the before mentioned theories refer to spherical colloidal particles and polymers. Our system is different in that it contains spheres and rod particles. Such a sphere-rod system has been theoretically addressed by Mao et al., ${ }^{22,24}$ who attribute stability to a repulsive 
energy barrier, i.e. a kinetic effect. This theory unfortunately does not cover the ratio of sphere size and rod length in our system, the concentration regime of fibrils (our system is in a nematic regime above $0.4 \%{ }^{59}$ ), nor the semi-flexibility.

The issue of thermodynamic versus kinetic stability remains intriguing, but its final resolution has to be deferred to further experimental work, as also commented on by Lu et al.. ${ }^{38}$ However, we note that the mixing of emulsions and fibrils at higher fibril concentration and the disaggregation upon dilution both needed mild agitation. This points towards a kinetic picture more than a thermodynamic one. Based on the DWS measurements we conclude that a diffusive motion still takes place at higher fibril concentrations. Although the diffusivity is slowed down at higher fibril concentrations this slowing down is only a small effect (cf. Figure 5c). Since the autocorrelation decays to zero we do not expect that the diffusing droplets/aggregates are confined to a cage with a size in the order of their own dimension. The decreasing $p$-value implies that the matrix develops visco-elastic properties. These results suggest that the oil droplets/aggregates are relatively free to diffuse over shorter length scales and are trapped on larger length scales as determined by the elastic properties of the fibril containing matrix. This is supported by the DWS measurements ( $p$ value, cf. Figure $5 b$ ) and the fact that we do not see creaming. In our opinion this supports the view that the emulsion is kinetically stabilized at higher fibril concentration.

\subsection{Conclusions}

We have observed that oil in water emulsions with droplet size of micrometers and fibrils of micrometer length in the water phase form stable emulsions above a certain fibril concentration. Above this concentration, the size of the droplet aggregates becomes independent of protein fibril concentration. The efficiency of stabilization could be improved by increasing the average length of fibrils. We found that longer fibrils are more efficient in stabilizing the emulsions, and that the stabilization is found independent of oil volume fraction and temperature. No fibril gel network or droplet gel network was formed in the emulsions, and viscosity of the continuous phase did not explain the stability. The issue of thermodynamic versus kinetic stability of aggregates has been discussed and our results point towards the mechanism of a kinetic stabilization. The findings could be of importance to research and applications of semi-flexible polymers in colloidal dispersions. Regardless the mechanism of stabilization it is noteworthy that the use of long fibrils allow 
to stabilize an emulsion, while remaining a low-viscosity fluid, enabling stabilization of e.g. drinkable emulsions.

\subsection{Acknowledgement}

This research is financially supported by NanoNextNL (consortium of Dutch government and 130 other partners). We thank Harry Baptist (WUR) for helping the TEM analysis. 


\subsection{References}

1. C. Veerman, G. de Schiffart, L. M. C. Sagis and E. van der Linden, International Journal of Biological Macromolecules, 2003, 33, 121-127.

2. L. N. Arnaudov and R. de Vries, Biophysical Journal, 2005, 88, 515-526.

3. C. Akkermans, A. J. Van der Goot, P. Venema, H. Gruppen, J. M. Vereijken, E. Van der Linden and R. M. Boom, Journal of Agricultural and Food Chemistry, 2007, 55, 9877-9882.

4. C. Akkermans, A. J. van der Goot, P. Venema, E. van der Linden and R. M. Boom, Food Hydrocolloids, 2008, 22, 1315-1325.

5. C. D. Munialo, A. H. Martin, E. van der Linden and H. H. J. de Jongh, Journal of Agricultural and Food Chemistry, 2014, 62, 2418-2427.

6. P. Aymard, T. Nicolai, D. Durand and A. Clark, Macromolecules, 1999, 32, 2542-2552.

7. C. Akkermans, P. Venema, A. J. van der Goot, H. Gruppen, E. J. Bakx, R. M. Boom and E. van der Linden, Biomacromolecules, 2008, 9, 1474-1479.

8. G. M. Kavanagh, A. H. Clark and S. B. Ross-Murphy, International Journal of Biological Macromolecules, 2000, 28, 41-50.

9. E. H. C. Bromley, M. R. H. Krebs and A. M. Donald, Faraday Discussions, 2005, 128, 13-27.

10. L. M. C. Sagis, C. Veerman and E. van der Linden, Langmuir, 2004, 20, 924-927.

11. J. Adamcik, J.-M. Jung, J. Flakowski, P. De Los Rios, G. Dietler and R. Mezzenga, Nature nanotechnology, 2010, 5, 423-428.

12. A. P. Philipse, Langmuir, 1996, 12, 1127-1133.

13. R. Tuinier, J. Rieger and C. G. de Kruif, Advances in Colloid and Interface Science, 2003, 103, 131.

14. S. Asakura and F. Oosawa, The Journal of Chemical Physics, 1954, 22, 1255-1256.

15. S. Asakura and F. Oosawa, Journal of Polymer Science, 1958, 33, 183-192.

16. R. I. Feigin and D. H. Napper, Journal of Colloid and Interface Science, 1980, 75, 525-541.

17. C. Sun, S. Gunasekaran and M. P. Richards, Food Hydrocolloids, 2007, 21, 555-564.

18. G. A. Vliegenthart and H. N. W. Lekkerkerker, The Journal of Chemical Physics, 1999, 111, 41534157.

19. K. H. Lin, J. C. Crocker, A. C. Zeri and A. G. Yodh, Physical Review Letters, 2001, 87, 088301.

20. H. N. W. Lekkerkerker and R. Tuinier, Colloids and the depletion interaction, Springer, Dordrecht ; New York, 2011.

21. T. B. J. Blijdenstein, C. Veerman and E. van der Linden, Langmuir, 2004, 20, 4881-4884.

22. Y. Mao, M. E. Cates and H. N. W. Lekkerkerker, Physical Review Letters, 1995, 75, 4548-4551.

23. X. Zhang, M. R. Servos and J. Liu, Journal of the American Chemical Society, 2012, 134, 99109913. 
24. Y. Mao, M. E. Cates and H. N. W. Lekkerkerker, Physica A: Statistical Mechanics and its Applications, 1995, 222, 10-24.

25. G. H. Koenderink, D. G. A. L. Aarts and A. P. Philipse, Journal of Chemical Physics, 2003, 119, 4490-4499.

26. P. R. Lang, Journal of Chemical Physics, 2007, 127, 124906.

27. W. H. Li, T. Yang and H. R. Ma, Journal of Chemical Physics, 2008, 128, 044910.

28. D. Guu, J. K. Dhont, G. A. Vliegenthart and M. P. Lettinga, J Phys Condens Matter, 2012, 24, 464101.

29. C. O. Klein van, P. Venema, L. M. C. Sagis, D. v. Dusschoten, M. Wilhelm, H. W. Spiess, E. v. d. Linden, S. S. Rogers and A. M. Donald, Applied rheology, 2007, 17, 45210.

30. S. S. Rogers, P. Venema, L. M. C. Sagis, E. van der Linden and A. M. Donald, Macromolecules, 2005, 38, 2948-2958.

31. T. B. J. Blijdenstein, W. P. G. Hendriks, E. van der Linden, T. van Vliet and G. A. van Aken, Langmuir, 2003, 19, 6657-6663.

32. H. M. Wyss, S. Romer, F. Scheffold, P. Schurtenberger and L. J. Gauckler, Journal of Colloid and Interface Science, 2001, 241, 89-97.

33. T. G. Mason, Rheologica Acta, 2000, 39, 371-378.

34. S. G. Bolder, International dairy journal, 2007, 17, 846-853.

35. L. N. Arnaudov, R. de Vries, H. Ippel and C. P. M. van Mierlo, Biomacromolecules, 2003, 4, 16141622.

36. A. Breugem, F. Bouchama and G. Koper, Surface Coatings International Part B: Coatings Transactions, 2005, 88, 135-138.

37. E. ten Grotenhuis, M. Paques and G. A. van Aken, Journal of Colloid and Interface Science, 2000, 227, 495-504.

38. P. J. Lu, J. C. Conrad, H. M. Wyss, A. B. Schofield and D. A. Weitz, Physical Review Letters, 2006, 96, 028306.

39. A. Sharma, S. N. Tan and J. Y. Walz, Journal of Colloid and Interface Science, 1997, 190, 392407.

40. M. Yasrebi, W. Y. Shih and I. A. Aksay, Journal of Colloid and Interface Science, 1991, 142, 357368.

41. S. Kim, K. Hyun, J. Y. Moon, C. Clasen and K. H. Ahn, Langmuir, 2015, 31, 1892-1900.

42. C. Cowell, R. Li-In-On and B. Vincent, Journal of the Chemical Society, Faraday Transactions 1: Physical Chemistry in Condensed Phases, 1978, 74, 337-347.

43. B. Vincent, P. F. Luckham and F. A. Waite, Journal of Colloid and Interface Science, 1980, 73, 508-521.

44. J. Clarke and B. Vincent, Journal of the Chemical Society, Faraday Transactions 1: Physical Chemistry in Condensed Phases, 1981, 77, 1831-1843. 
45. J. Clarke and B. Vincent, Journal of Colloid and Interface Science, 1981, 82, 208-216.

46. B. Vincent, J. Edwards, S. Emmett and A. Jones, Colloids and Surfaces, 1986, 18, 261-281.

47. J. M. H. M. Scheutjens and G. J. Fleer, Advances in Colloid and Interface Science, 1982, 16, 361380.

48. G. Fleer and J. Scheutjens, The stability of dispersions of hard spherical particles in the presence of nonadsorbing polymer, 1984.

49. G. J. Fleer and J. M. H. M. Scheutjens, Croatica chemica acta, 1987, 60, 477-494.

50. A. P. Gast and L. Leibler, The Journal of Physical Chemistry, 1985, 89, 3947-3949.

51. A. P. Gast and L. Leibler, Macromolecules, 1986, 19, 686-691.

52. Y.-L. Chen and K. S. Schweizer, The Journal of Chemical Physics, 2002, 117, 1351-1362.

53. A. L. Ogden and J. A. Lewis, Langmuir, 1996, 12, 3413-3424.

54. J. Y. Walz and A. Sharma, Journal of Colloid and Interface Science, 1994, 168, 485-496.

55. J. Liu, W. Y. Shih, R. Kikuchi and I. A. Aksay, Journal of Colloid and Interface Science, 1991, 142, 369-377.

56. A. Jones and B. Vincent, Colloids and Surfaces, 1989, 42, 113-138.

57. N. Dutta and D. Green, Langmuir, 2008, 24, 5260-5269.

58. J. Groenewold and W. K. Kegel, The Journal of Physical Chemistry B, 2001, 105, 11702-11709.

59. R. Mezzenga, J.-M. Jung and J. Adamcik, Langmuir, 2010, 26, 10401-10405. 


\subsection{Appendices}

\section{Appendix A}

Table A.1 The volume fraction of fibrils as a function of WPI weight concentration.

\begin{tabular}{|l|l|}
\hline WPI concentration (wt \%) & Fibril volume fraction $(\varphi)$ \\
\hline 0.001 & $3.75 \times 10^{-6}$ \\
\hline 0.005 & $1.87 \times 10^{-5}$ \\
\hline 0.01 & $3.75 \times 10^{-5}$ \\
\hline 0.05 & $1.87 \times 10^{-4}$ \\
\hline 0.1 & $3.75 \times 10^{-4}$ \\
\hline 0.5 & $1.87 \times 10^{-3}$ \\
\hline 1 & $3.75 \times 10^{-3}$ \\
\hline 1.5 & $5.62 \times 10^{-3}$ \\
\hline 2 & $7.50 \times 10^{-3}$ \\
\hline 2.25 & $8.43 \times 10^{-3}$ \\
\hline 2.5 & $9.37 \times 10^{-3}$ \\
\hline 2.75 & $1.03 \times 10^{-2}$ \\
\hline 3 & $1.12 \times 10^{-2}$ \\
\hline 3.25 & $1.22 \times 10^{-2}$ \\
\hline 4 & $1.50 \times 10^{-2}$ \\
\hline
\end{tabular}




\section{Appendix B}
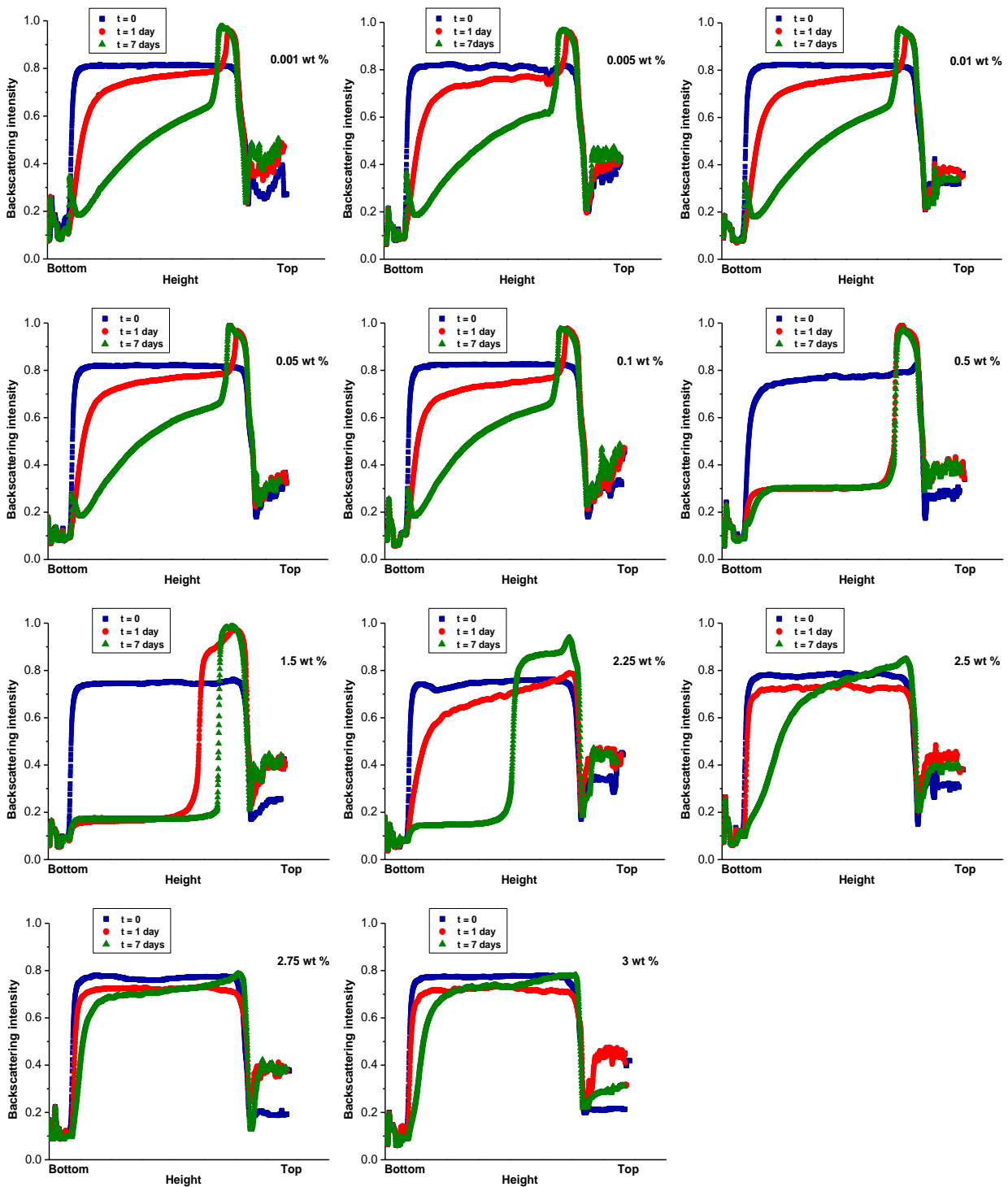

Figure B.1 Backscattering intensity profiles of samples containing $10 \%(\mathrm{v} / \mathrm{v}) \mathrm{o} / \mathrm{w}$ emulsion and $0.001,0.005,0.01,0.05,0.1,0.5,1.5,2.25,2.5,2.75$ or $3 \mathrm{wt} \%$ protein fibrils. The horizontal axis represents the position along the tube. 


\section{Chapter 7}

\section{Stability of colloidal dispersions in} the presence of protein fibrils

Published as:

Jinfeng Peng, Ardy Kroes-Nijboer, Paul Venema, Erik van der Linden. Stability of colloidal dispersions in the presence of protein fibrils. Soft Matter, 2016, 12, 3514-3526. 


\section{Abstract}

We studied the stability of monodispersed polystyrene latex dispersions with protein fibrils at different concentrations at $\mathrm{pH} 2$ using microscopy and diffusing wave spectroscopy. At low fibril concentrations, fibrils induced bridging flocculation due to the opposite charges between fibrils and the latex particles. At higher fibril concentration the dispersions were stabilized due to steric and/or electrostatic repulsion. Upon further increasing fibril concentration, we find that the dispersion is destabilized again by depletion interaction. At even higher fibril concentration, the dispersions are stabilized again. These dispersions have a higher stability compared to the dispersions without fibrils. Interestingly, these dispersions contain single particles and small clusters of particles that do not grow beyond a certain size. Although the stabilization mechanism is not clear yet, the results from microscopy and diffusing wave spectroscopy point in the direction of a kinetic barrier that depends on fibril concentration. 


\subsection{Introduction}

Protein fibrils are semi-flexible rod-like aggregates that can be formed from various proteins under certain conditions. ${ }^{1-3} \beta$-Lactoglobulin ( $\beta$-lg), a globular whey protein, forms fibrils when it is heated at $\mathrm{pH} 2$ and elevated temperature. ${ }^{4} 5$ During this process $\beta$-lg is hydrolyzed into small peptides, and part of these peptides assembles into fibrils. ${ }^{6}$ The fibrils that are formed are several micrometers long, but only a few nanometers thick. ${ }^{7}$ This high aspect ratio makes them interesting candidates as, e.g. thickeners or stabilizers in industrial applications. ${ }^{8}$ Gel formation at very low concentration of fibrils has also been reported. ${ }^{9}$ In addition, fibrils can be used in the production of microcapsules, where fibrils were found to increase the strength of the capsules. ${ }^{10-12}$ The main focus of the studies on protein fibrils has been on their formation and on the properties of fibril dispersions. Only a limited number of studies focused on the behavior of more complex systems containing protein fibrils. Here we focus on the stability of colloidal dispersions containing fibrils.

Colloidal dispersions are present in a wide variety of products. Their use ranges from foods to paints and pharmaceutical products. The stability of colloidal dispersions can be affected by the addition of polymers. ${ }^{13}$ In particular, extensive theoretical and experimental work has been performed on the phase behavior of rod-like particles in colloidal dispersions and rods are found to induce rich phase behavior in colloidal dispersions. $^{14-24}$

Blijdenstein et al. studied the behavior of $\beta$-Ig fibrils in oil-in-water emulsions and found that fibrils can induce flocculation of the emulsion droplets. In their study, both fibrils and emulsion droplets are positively charged and the flocculation was attributed to depletion interaction. $^{25}$ However, in the case that fibrils and emulsion droplets are oppositely charged, one may observe, depending on the fibril concentration, bridging flocculation.

In our study a monodisperse polystyrene latex dispersion was used as a model for a colloidal system. The average fibril length in the current study is larger than the diameter of the latex particles. We used diffusing wave spectroscopy (DWS) to monitor the diffusive motion of the particles at various fibril concentrations. DWS is a suitable technique since fibril dispersions are transparent and only the particles scatter light. We studied the influence of size and surface charge of the particles on the stability of the dispersions containing fibrils. 


\subsection{Materials and methods}

\subsubsection{Materials}

Whey protein isolate (WPI, Bipro) was supplied by Davisco (Lot \# 198-1-420), USA. Lysozyme from chicken egg white was obtained from Fluka, Belgium. Polystyrene latex dispersions used in this experiment were obtained from Thermo Scientific Optibind, CA. The latex consists of monodisperse polystyrene spheres with a diameter of either $208 \mathrm{~nm}$ or $862 \mathrm{~nm}$. The solid content of the latex dispersions is $10 \%(\mathrm{w} / \mathrm{w})$ solids. The concentration of both the fibril solution and polystyrene latex particles are given in weight percentage.

\subsubsection{Fibril formation}

For the preparation of protein fibrils, we have followed the procedure as described previously. ${ }^{26}$ In short, a stock solution of $7.5 \mathrm{wt} \% \mathrm{WPI}$ was prepared by dissolving the protein powder in a $\mathrm{HCl}$ solution of $\mathrm{pH}$ 2. The solution was left overnight in the cold room $\left(4^{\circ} \mathrm{C}\right)$ while mildly stirred. The $\mathrm{pH}$ of the protein solution was adjusted to $\mathrm{pH} 2$ using a $6 \mathrm{M}$ $\mathrm{HCl}$ solution, followed by a filtration through a protein filter (Hydrophilic PES $0.45 \mu \mathrm{m}$, Millipore Millex-HP) to remove undissolved protein. Subsequently, the protein concentration of the stock solution was determined using a UV spectrophotometer (Cary 50 Bio, Varian) and a calibration curve of known WPI concentration at a wavelength of 278 $\mathrm{nm}$. The stock solution was diluted to a protein concentration of $4 \mathrm{wt} \%$ with a $\mathrm{HCl}$ solution of $\mathrm{pH}$ 2. The diluted solution was heated in a heating and stirring plate at $80{ }^{\circ} \mathrm{C}$ for 22 hours, while mildly stirring during heating, using a magnetic stirring bar. After heating, the fibril solution was cooled in ice-water and kept at $4{ }^{\circ} \mathrm{C}$ before use. The fibril concentration is given in terms of the total amount of WPI dissolved before heating.

\subsubsection{Length distribution of fibrils}

We used flow-induced birefringence to determine the length distribution of the fibrils. After the cessation of flow, the decay curves of the flow-induced birefringence were measured with a strain-controlled ARES rheometer (Rheometrics Scientific) equipped with a modified optical analysis module. ${ }^{27}$ The length distribution of the fibrils was determined from these decay curves. ${ }^{28}$ 


\subsubsection{Morphology of protein fibrils}

Transmission electron microscopy (TEM) was used to determine the morphology of protein fibrils. The sample was diluted 400 -fold before imaging. After dilution a droplet of sample was placed on a carbon support film on a copper grid. After $15 \mathrm{~s}$, the droplet was removed with a filter paper. Subsequently, a droplet of $2 \%$ uranyl acetate was put onto the grid and removed after $15 \mathrm{~s}$. Electron micrographs were taken using a transmission electron microscope (JEOL JEM-1011, Tokyo, Japan).

\subsubsection{Surface modification of latex particles}

To adjust the surface charge, the latex particles were coated using lysozyme. The lysozyme was dissolved in an $\mathrm{HCl}$ solution at $\mathrm{pH} 2$ and mixed with the latex particles. The mixtures were incubated overnight at $4{ }^{\circ} \mathrm{C}$. Subsequently, the mixtures were centrifuged at $16.000 \mathrm{~g}$ for 30 minutes to remove the non-adsorbed lysozyme. Three washing steps were performed and the supernatant was removed after each centrifugation step. The precipitates were re-suspended using an $\mathrm{HCl}$ solution at $\mathrm{pH} 2$ to the initial volume. The zeta potential of washed latex particles was measured to confirm the surface coverage of particles with lysozyme. At pH 2 the latex particles had a zeta potential of - $60 \mathrm{mV}$.

\subsubsection{Zeta potential}

The zeta potential of the latex particles was determined using a Zetasizer Nano (Zetasizer Nano, Malvern Instruments). The samples were diluted 100 -fold using an $\mathrm{HCl}$ solution of $\mathrm{pH} 2$ and the zeta potential was calculated from the mobility values using the Smoluchowski model. Five measurements were performed for each sample and the average number was used.

\subsubsection{Scanning Electron Microscopy (SEM)}

The morphology of latex particles with diameter of $862 \mathrm{~nm}$ was characterized using SEM. A small droplet of the sample suspension (0.01 wt\%) was put on Nuclepore Polycarbonate membrane ( $1 \mu \mathrm{m}$ holes, Costar) which was placed on a filter paper to absorb the fluid through the membrane leaving the sample on the top side. After air drying the membrane were glued on sample holders by carbon adhesive tabs (EMS, Washington, USA) and 
sputter coated with $10 \mathrm{~nm}$ Iridium (Leica EM SCD 500, Vienna, Austria). Samples were analyzed at $2 \mathrm{kV}$ at room temperature in a high resolution scanning electron microscope (Magellan 400, FEI, Eindhoven, The Netherlands).

\subsubsection{Sample preparation}

Three latex dispersions were used to mix with fibrils depending on the size and the surface properties of the latex particles: 1) latex dispersions containing particles with a diameter of $208 \mathrm{~nm}$; 2) latex dispersions containing particles with a diameter of $862 \mathrm{~nm}$; 3) latex dispersions containing lysozyme-coated particles with a diameter of $862 \mathrm{~nm}$.

In all fibril-particle mixtures, 1 wt\% latex particles were mixed with protein fibrils at different concentrations. A concentration range of protein fibrils was prepared by diluting the stock fibril solution of 4 wt\%. The final concentration of the protein fibrils in the mixture is $0,0.001,0.0025,0.005,0.0075,0.01,0.025,0.05,0.1,0.5,1,1.5,2,2.5,3$ or 3.5 $w t \%$. The fibril concentration is given in terms of the total amount of WPI dissolved before heating.

\subsubsection{Visual observation}

Dispersions were kept at both $20{ }^{\circ} \mathrm{C}$ and $65{ }^{\circ} \mathrm{C}$, images were taken directly after preparation and 1 hour, 1 day, 5 days and 14 days after preparation.

\subsubsection{Optical microscopy images}

Dispersions were imaged using optical microscopy (Axioskop Zeiss, Germany) equipped with a CCD camera (AxioCam HRC, Zeiss). The bottom layer of the dispersions after 7 days of preparation at $20^{\circ} \mathrm{C}$ was taken for imaging.

\subsubsection{Diffusing wave spectroscopy (DWS)}

The latex particles with fibrils were analysed at $20^{\circ} \mathrm{C}$ directly after mixing using DWS in transmission mode. In DWS, we measure the intensity autocorrelation function $g_{2}(t)$ of the multiple scattered light. The $g_{2}(t)$ is related to the mobility of the latex particles according to the reference ${ }^{29}$ as 


$$
g_{2}(t)-1=\beta\left(\int_{0}^{\infty} P(s) \exp \left(-1 / 3 k_{0}^{2}<\Delta r^{2}(t)>\frac{s}{l^{*}}\right) d s\right)^{2}
$$

where, on the basis of the Siegert relation that

$$
g_{2}(t)-1=\beta\left|g_{1}(t)\right|^{2}
$$

Here $\beta$ is a constant determined by the collection optics, $P(s)$ is the path length distribution function of photons in the sample, $k_{0}=2 \pi n / \lambda$, with $n$ the refractive index of the solvent and $\lambda$ the wavelength of the laser, $\left\langle\Delta r^{2}(t)>\right.$ is the mean square displacement of the latex particles, and $I^{*}$ is the photo transport mean free path.

By numerically inverting the correlation functions and using Equation 1, we can obtain the mean square displacement (MSD) $\left\langle\Delta \mathrm{r}^{2}(\mathrm{t})>\right.$, which is an expression for the motion of the latex particles. We define $Q^{2}=\left(\frac{L k_{0}}{l^{*}}\right)^{2}\left\langle\Delta r^{2}(t)\right\rangle$. $\mathrm{L}$ is the optical path length through the sample cell. The latex particles would undergo Brownian motion (unhindered diffusive colloidal motion) in a purely viscous solution, and $Q^{2}$ would vary linearly with time $Q^{2} \sim D t$, where $D$ is the diffusion constant. In a viscoelastic medium the short time behavior of $Q^{2}$ can generally be defined by a power law $\left\langle Q^{2}(t)>\sim D t^{p}\right.$ where $\mathrm{D}$ is the diffusive constant, $\mathrm{p}$ is a measure for the viscoelastic properties of the matrix. The matrix is purely viscous when $\mathrm{p}=1$. The matrix behaves like a viscous medium $\left(\mathrm{G}^{\prime}<\mathrm{G}^{\prime \prime}\right)$ when $\frac{1}{2}<p<$ 1 and behaves like an elastic medium $\left(G^{\prime}>G^{\prime \prime}\right)$ When $p \leq \frac{1}{2}{ }^{30}$ The half-life time of the decorrelation, $\tau_{1 / 2}$, was defined when $g_{2}\left(\tau_{1 / 2}\right)-1=\frac{1}{2} .{ }^{31}$

A He-Ne laser ( $20 \mathrm{~mW}$ ) was used operating at $\lambda=633 \mathrm{~nm}$. The laser beam was expanded with a beam expander to $3 \mathrm{~mm}$. A transparent glass cell with a path length of $2 \mathrm{~mm}$ was used. The laser light that passed the glass cell was collected in a single-mode optical fibre. We used polarizers to assure that solely the multiple scattered light was collected. The signal was split into two signals and directed into two photomultiplier tubes (ALV/SO-SIPD, Germany). The output of the two tubes was cross-correlated using a multiple tau digital correlator (ALV-5000/60X0, Germany). The dispersions were monitored for 3 minutes for every measurement. The intensity autocorrelation function is determined by averaging 10 measurements. 


\subsection{Results and Discussion}

\subsubsection{Characterization}

The length concentration distribution $C_{L}$ as determined using flow-induced birefringence, and a TEM image of the fibril solution, is shown in Figure $1 \mathrm{a}$ and $b$. The SEM image of a latex particle with diameter of $862 \mathrm{~nm}$ is shown in Figure 2.

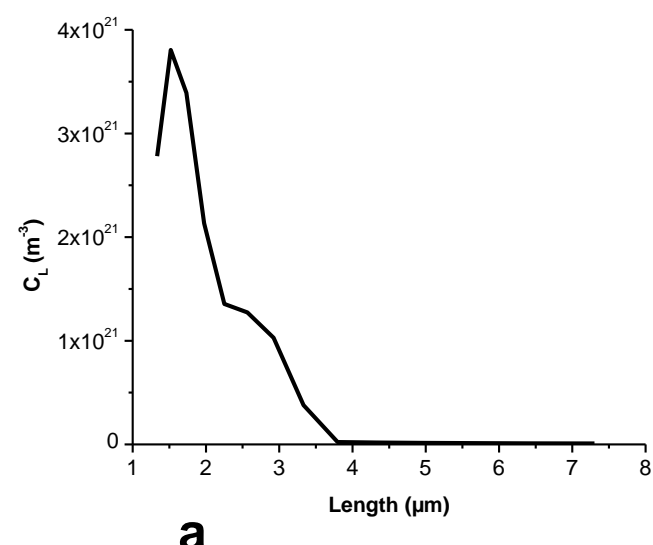

a

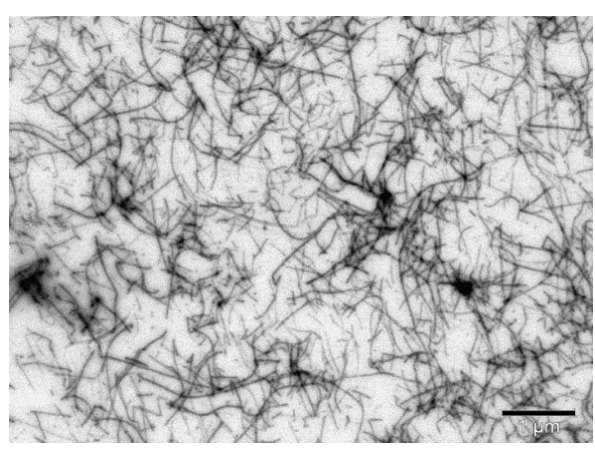

b

Figure 1 (a):The length concentration distribution $\mathrm{CL}$ of the protein fibrils prepared by heating a 4 wt\% WPI solution for 22 hours at $80^{\circ} \mathrm{C}$ and $\mathrm{pH}$ 2. (b): The corresponding TEM image of protein fibrils. The scale bar corresponds to $1 \mu \mathrm{m}$. 


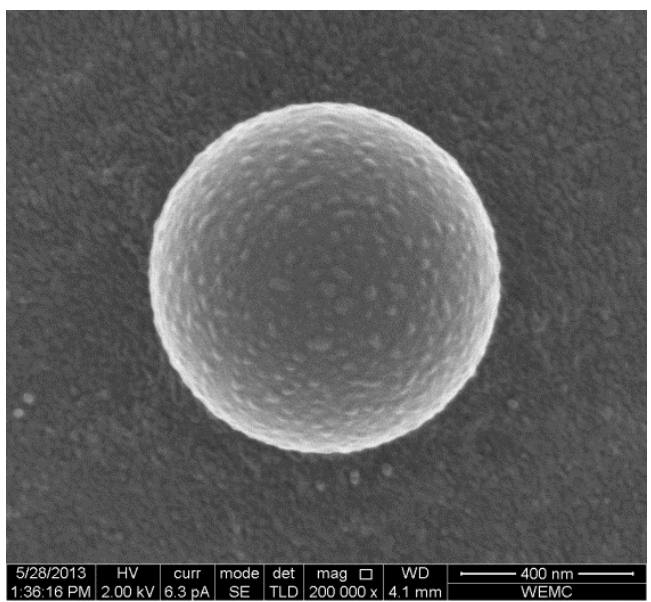

Figure 2 SEM image of a latex particle with diameter of $862 \mathrm{~nm}$. The scale bar corresponds to 400 $\mathrm{nm}$.

The fibrils are polydisperse and semi-flexible as shown in Figure $1 \mathrm{a}$. We estimate the ratio of fibril length $L$ to diameter $D$ as $L / D=500$. The fibrils are positively charged at $p H$ 2. ${ }^{4} \mathrm{WPI}$ contains about $65 \%(\mathrm{w} / \mathrm{w})$ of $\beta-\mathrm{lg}$ and this protein is the main contributor to the formation of fibrils. ${ }^{32}$ The conversion rate of protein into fibrils under the conditions used here was determined to be about $58 \% .^{33}$ As a result, 38\% (w/w) of the total amount of protein is converted into fibrils. Previous research has shown that peptides are the building blocks of fibrils, ${ }^{6}$ to estimate the volume fraction of the fibrils, we assume that one peptide per protein is incorporated in the fibrils. The volume per peptide is estimated as $\frac{\pi}{4} D^{2} l$, where the diameter $D$ of a fibril is taken as $4 \mathrm{~nm}^{34}$ and the length per peptide $l$ is taken as $2.4 \mathrm{~nm} .^{6}$ The resulting volume fraction of fibrils as a function of protein concentration is listed in Table 1. 
Table 1 The fibril volume fraction as a function of WPI concentration

\begin{tabular}{|c|c|}
\hline $\begin{array}{l}\text { WPI concentration } \\
\text { (wt } \%)\end{array}$ & $\begin{array}{l}\text { Fibril volume fraction } \\
\qquad(\phi)\end{array}$ \\
\hline 0.001 & $3.75 \times 10^{-6}$ \\
\hline 0.0025 & $9.37 \times 10^{-6}$ \\
\hline 0.005 & $1.87 \times 10^{-5}$ \\
\hline 0.0075 & $2.81 \times 10^{-5}$ \\
\hline 0.01 & $3.75 \times 10^{-5}$ \\
\hline 0.025 & $9.37 \times 10^{-5}$ \\
\hline 0.05 & $1.87 \times 10^{-4}$ \\
\hline 0.1 & $3.75 \times 10^{-4}$ \\
\hline 0.5 & $1.87 \times 10^{-3}$ \\
\hline 1 & $3.75 \times 10^{-3}$ \\
\hline 1.5 & $5.62 \times 10^{-3}$ \\
\hline 2 & $7.50 \times 10^{-3}$ \\
\hline 2.5 & $9.37 \times 10^{-3}$ \\
\hline 3 & $1.12 \times 10^{-2}$ \\
\hline 3.5 & $1.31 \times 10^{-2}$ \\
\hline 4 & $1.50 \times 10^{-2}$ \\
\hline
\end{tabular}

\subsubsection{Surface modification of latex particles}

Lysozyme is positively charged at $\mathrm{pH} 2^{35}$ and upon incubation lysozyme is expected to adsorb at the surface of the latex particles. ${ }^{36,37}$ After incubation the zeta potential of the washed latex particles (removing the non-adsorbed lysozyme) changed from $-60 \mathrm{mV}$ to $+56 \mathrm{mV}$, confirming the adsorption of lysozyme to the surface of latex particles. The schematic representation of the surface properties of latex particles used in this study is shown in Figure 3, showing the bare latex particles and lysozyme-coated latex particles at $\mathrm{pH} 2$. 


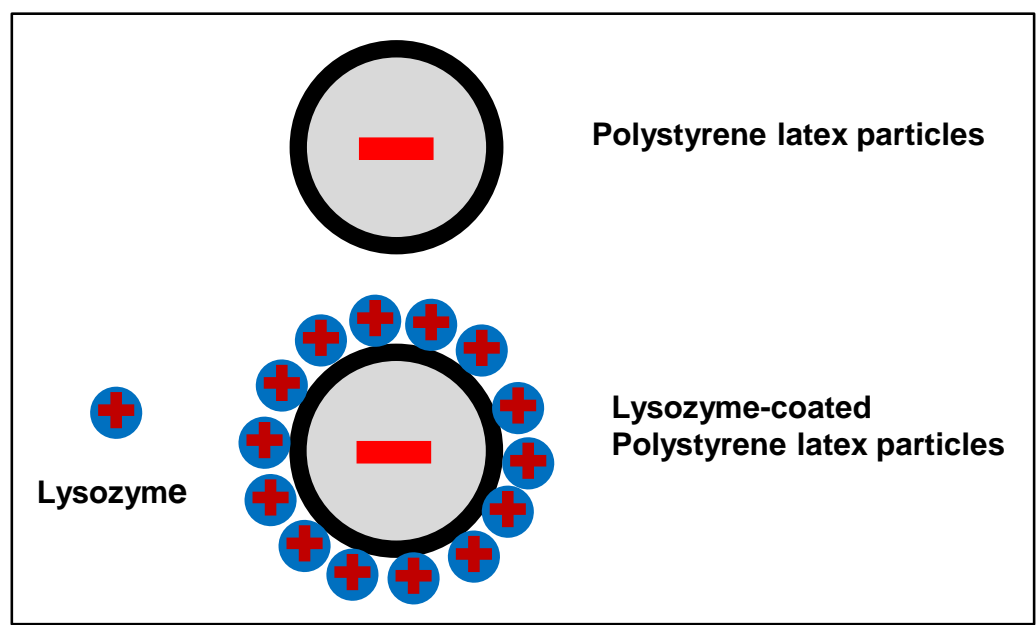

Figure 3 Schematic representation of the surface coverage of latex particles with diameter of 862 $\mathrm{nm}$ : bare latex particles and lysozyme-coated latex particles.

\subsubsection{Macroscopic stability of the latex dispersions}

The macroscopic stability of the latex dispersions mixed with fibrils is shown in Figure 4. Images were taken directly after sample preparation, 1 hour, 1 day, 5 days and 14 days. 


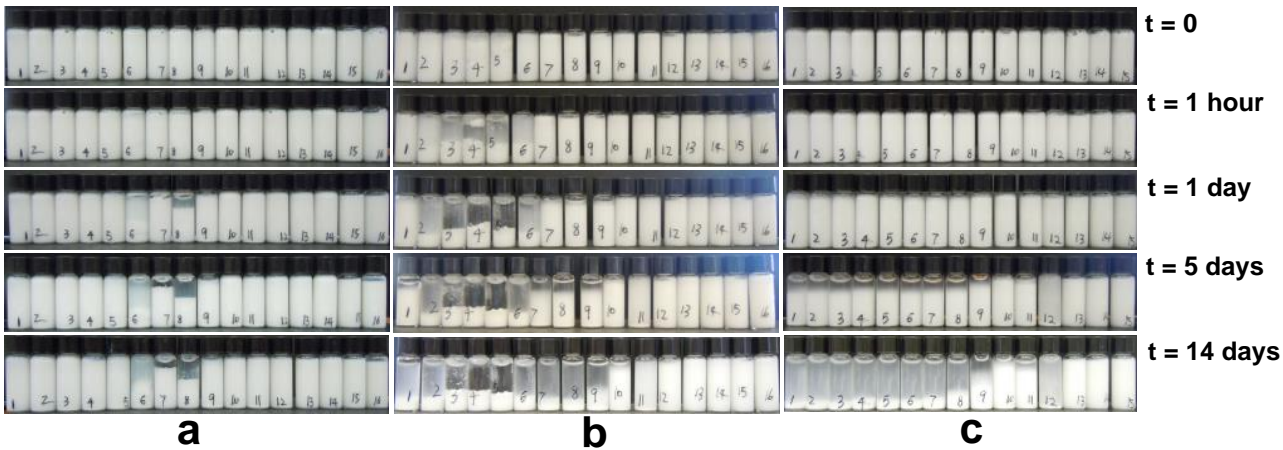

Figure 4 Images of 1 wt\% dispersions (at $20^{\circ} \mathrm{C}$ ) mixed with fibrils at concentrations of 0 (no fibrils added), $0.001,0.0025,0.005,0.0075,0.01,0.025,0.05,0.1,0.5,1,1.5,2,2.5,3$ or 3.5 wt\%. The fibrils concentration increases from left to right. The different rows represent the samples at different times after preparation. (a): dispersions containing latex particles with a diameter of 208 $\mathrm{nm}$. (b): dispersions containing latex particles with a diameter of $862 \mathrm{~nm}$. (c): dispersions containing lysozyme-coated latex particles with a diameter of $862 \mathrm{~nm}$. The sample with $3.5 \mathrm{wt} \%$ fibrils is not included in (c).

Images of dispersions containing particles with a diameter of $208 \mathrm{~nm}$ are shown in Figure 4 a. All dispersions with protein fibril concentrations up to 0.0075 wt $\%$ have comparable stability. Increasing the fibril concentration from $0.01 \mathrm{wt} \%$ up to $0.05 \mathrm{wt} \%$ the dispersions showed phase separation within 1 day, and to an increasing extent with increasing fibril concentration. After 5 days two clearly separated layers were observed. By further increasing the fibril concentration ( $\geq 0.1 \mathrm{wt} \%$ ), the dispersions remained stable for at least 14 days.

Images of dispersions containing particles with a diameter of $862 \mathrm{~nm}$ are shown in Figure $4 \mathrm{~b}$. The dispersions showed phase separation at fibril concentrations from 0.001 to 0.01 wt\% within 1 hour. Increasing the fibril concentration up to 0.1 wt\%, the stability of the dispersions increased and was comparable to the dispersion without fibrils. In dispersions with 1 wt\% fibrils, only limited sedimentation took place. At fibril concentrations above 1 $w t \%$, the samples were stable for at least 14 days. It is noted that these stabilized samples even showed higher stability than the sample without fibrils.

Images of dispersions containing lysozyme-coated particles with a diameter of $862 \mathrm{~nm}$ are shown in Figure 4 c. All samples were stable for at least 1 day. Samples containing up to 
0.1 wt\% phase separated within 14 days. At a fibril concentration of 0.5 wt\% the dispersion was stable. The dispersion phase separated again at fibril concentrations between $1 \mathrm{wt} \%$ and $1.5 \mathrm{wt} \%$. At fibril concentrations above $2 \mathrm{wt} \%$, the samples were stable for at least 14 days.

Based on the macroscopic observations, we identify the following 4 regimes with increasing fibril concentration: 1 ) at low fibril concentrations phase separation is observed; 2) above a critical fibril concentration, samples are stable; 3) increasing fibril concentration, dispersions show phase separation again; 4) further increasing the fibril concentration the dispersions are stable again.

\subsubsection{The effect of temperature on stability}

To investigate the influence of temperature on dispersions stability, dispersions with particle diameters of $208 \mathrm{~nm}$ and $862 \mathrm{~nm}$ were incubated at $65^{\circ} \mathrm{C}$ for 5 days (cf. Figure 5).

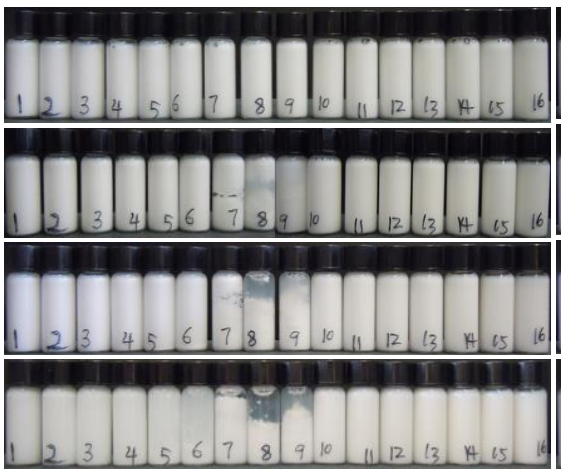

a
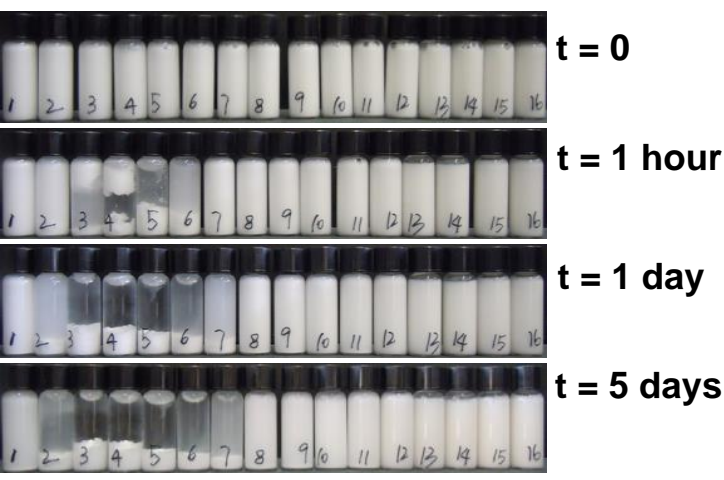

b

Figure 5 Images of 1 wt\% dispersions (at $65^{\circ} \mathrm{C}$ ) mixed with fibrils at concentrations of 0 (no fibrils added), $0.001,0.0025,0.005,0.0075,0.01,0.025,0.05,0.1,0.5,1,1.5,2,2.5,3$ or 3.5 wt\%. The fibrils concentration increases from left to right. The different rows represent the samples at different times after preparation. (a): dispersions containing latex particles with a diameter of 208 $\mathrm{nm}$. (b): dispersions containing latex particles with a diameter of $862 \mathrm{~nm}$.

The stability of dispersions with a particle diameter of $208 \mathrm{~nm}$ incubated at $65^{\circ} \mathrm{C}$ (cf. Figure 5 a), was almost identical to the stability at $20^{\circ} \mathrm{C}$ (cf. Figure 4 a). The only difference is the stability of the sample at $0.1 \mathrm{wt} \%$ of fibrils at $65^{\circ} \mathrm{C}$ (cf. Figure 5 a). In the case of 
dispersions with particles of $862 \mathrm{~nm}$ incubated at $65^{\circ} \mathrm{C}$, the stability was also almost identical to the stability at $20^{\circ} \mathrm{C}$ (cf. Figure $4 \mathrm{~b}$ ). Only the sample with $0.025 \mathrm{wt} \%$ fibrils at $65{ }^{\circ} \mathrm{C}$ showed more pronounced phase separation compared to the same sample at $20^{\circ} \mathrm{C}$ (cf. Figure $4 \mathrm{~b}$ ). At higher fibril concentration the dispersions were macroscopically stable. The results show that an increase in temperature only had a small influence on macroscopic stability.

\subsubsection{Microscopy}

To investigate the stability of the dispersions with fibrils, the samples were analysed after 7 days of sample preparation using microscopy. Samples of dispersions from the 4 identified regimes were imaged by optical microscopy. 

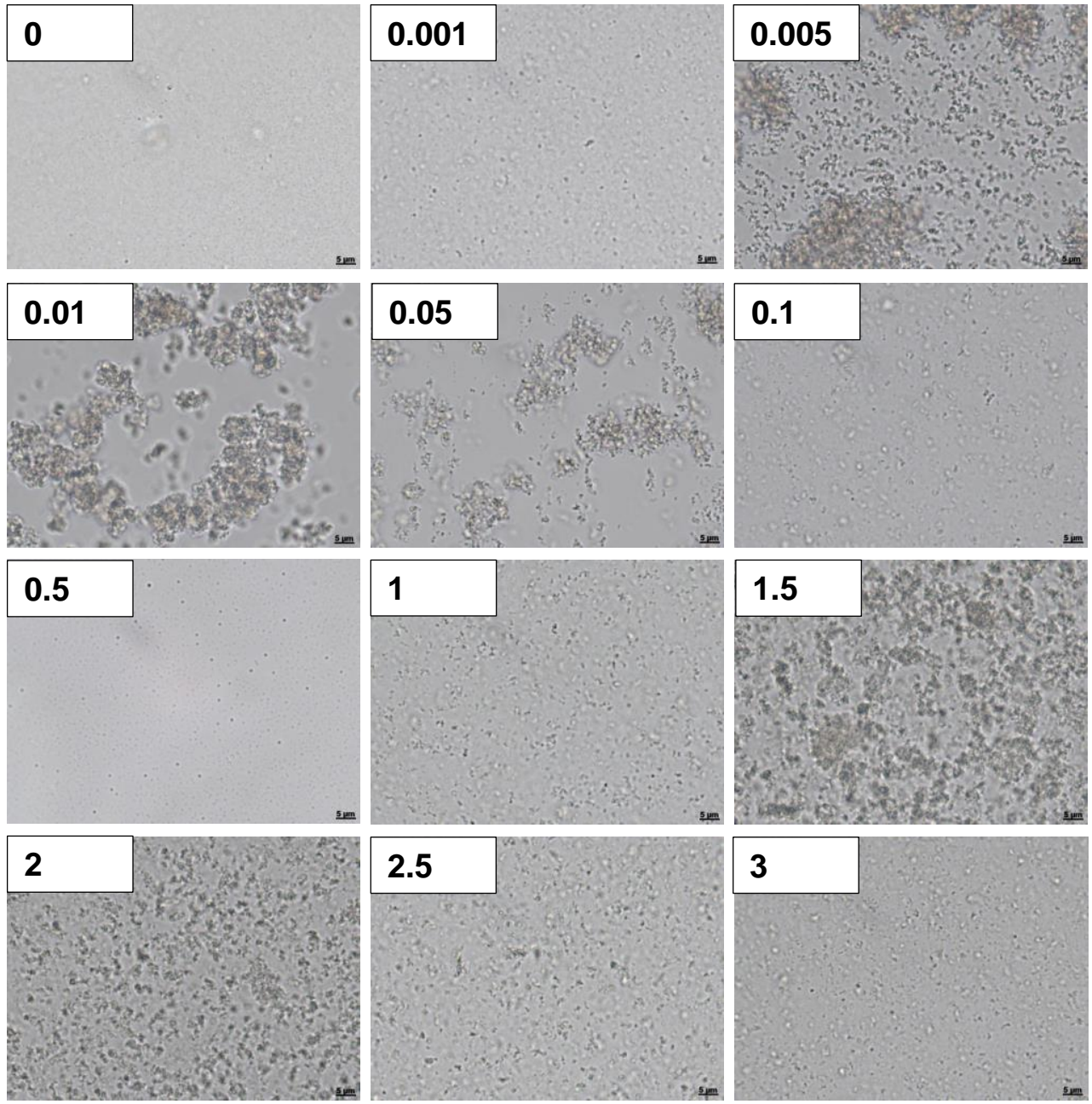

Figure 6 Microscopy images of the bottom layer of the dispersions at $20^{\circ} \mathrm{C}$ after 7 days of sample preparation. The particle diameter is $\mathbf{2 0 8} \mathbf{n m}$ and the fibril concentrations are $\mathbf{0}$ (no fibrils added), $0.001,0.005,0.01,0.05,0.1,0.5,1,1.5,2,2.5$ and $3 w t \%$. The fibril concentration (wt\%) is indicated in the images. The scale bar corresponds to $5 \mu \mathrm{m}$.

The images in Figure 6 show a complicated behaviour of aggregate size as a function of fibril concentration. In the dispersion without fibrils (0 wt\%) no particle aggregates could be observed. At low fibril concentrations ( $\leq 0.05$ wt\%), aggregates are observed, which increase in size with increasing fibril concentration. After further increasing the fibril concentration, the size of the aggregates decreases. At fibril concentrations higher than 
$0.1 \mathrm{wt} \%$, almost no aggregates could be observed anymore up to a fibril concentration of 1 wt\%. Upon further increasing the fibril concentration ( $\geq 1$ wt\%), we find particle aggregates again. The size of these aggregates reaches a maximal value at a certain fibril concentration and decreases again while increasing the fibril concentration. At higher fibril concentration ( $3 \mathrm{wt} \%$ ), the dispersions are even more stable than the dispersion without fibrils. In addition, we observed that small aggregates are present together with some single particles.
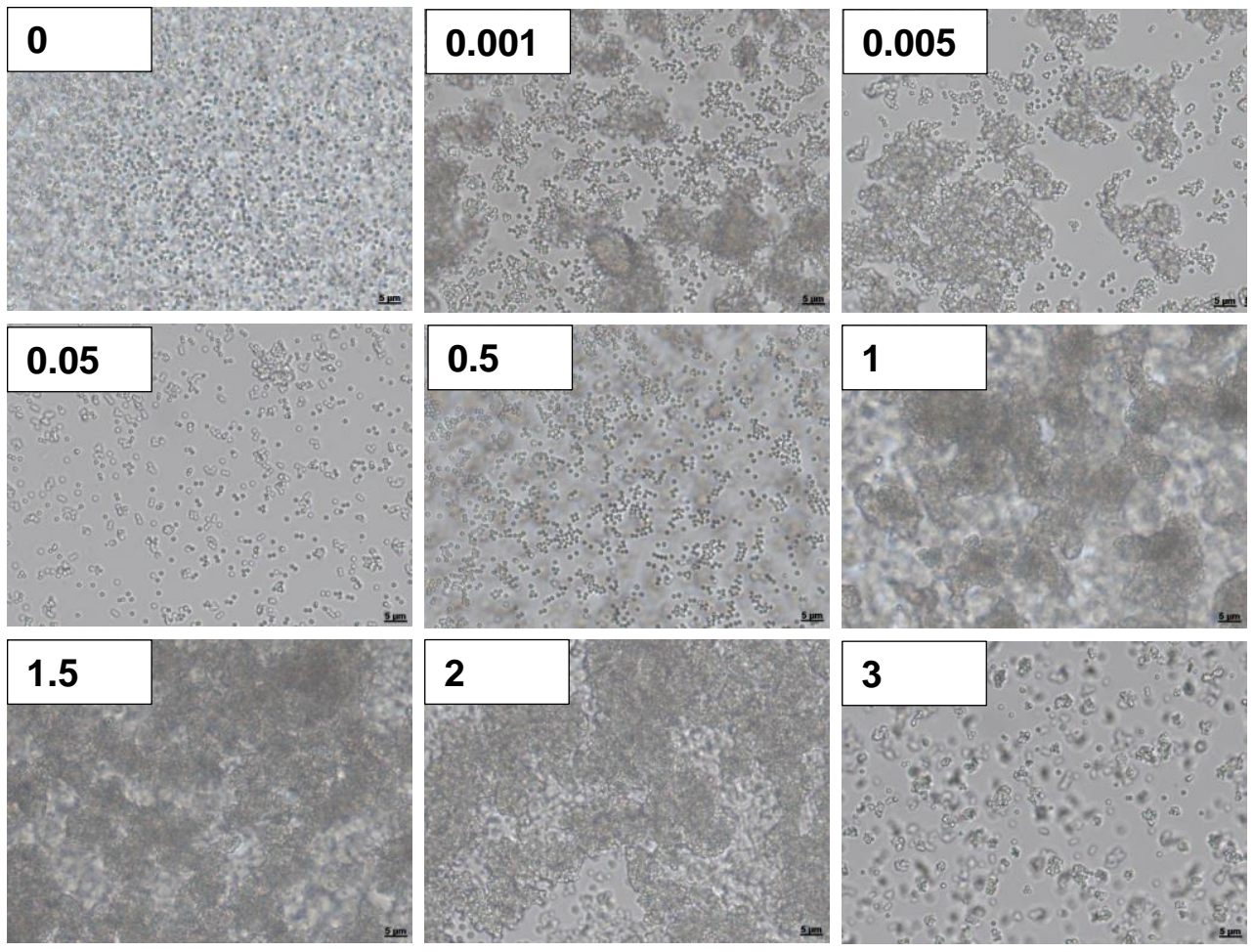

Figure 7 Microscopy images of the bottom layer of the dispersions at $20^{\circ} \mathrm{C}$ after 7 days of sample preparation. The particle diameter is $862 \mathrm{~nm}$ and the fibril concentrations are $\mathbf{0}$ (no fibrils added), $0.001,0.005,0.05,0.5,1,1.5,2$ and $3 w t \%$. The fibril concentration (wt\%) is indicated in the images. The scale bar corresponds to $5 \mu \mathrm{m}$.

The dispersions with a particle diameter of $862 \mathrm{~nm}$ (cf. Figure 7) show a similar behaviour as the dispersions with a particle diameter of $208 \mathrm{~nm}$ (cf. Figure 6). The main differences are the fibril concentrations at which the change in aggregate size starts. When larger 
particles are present the changes in aggregate size took place at lower fibril concentration. At higher fibril concentration ( 3 wt\%), the dispersions are even more stable than the dispersion without fibrils. In addition, we observed that small aggregates are present together with some single particles.
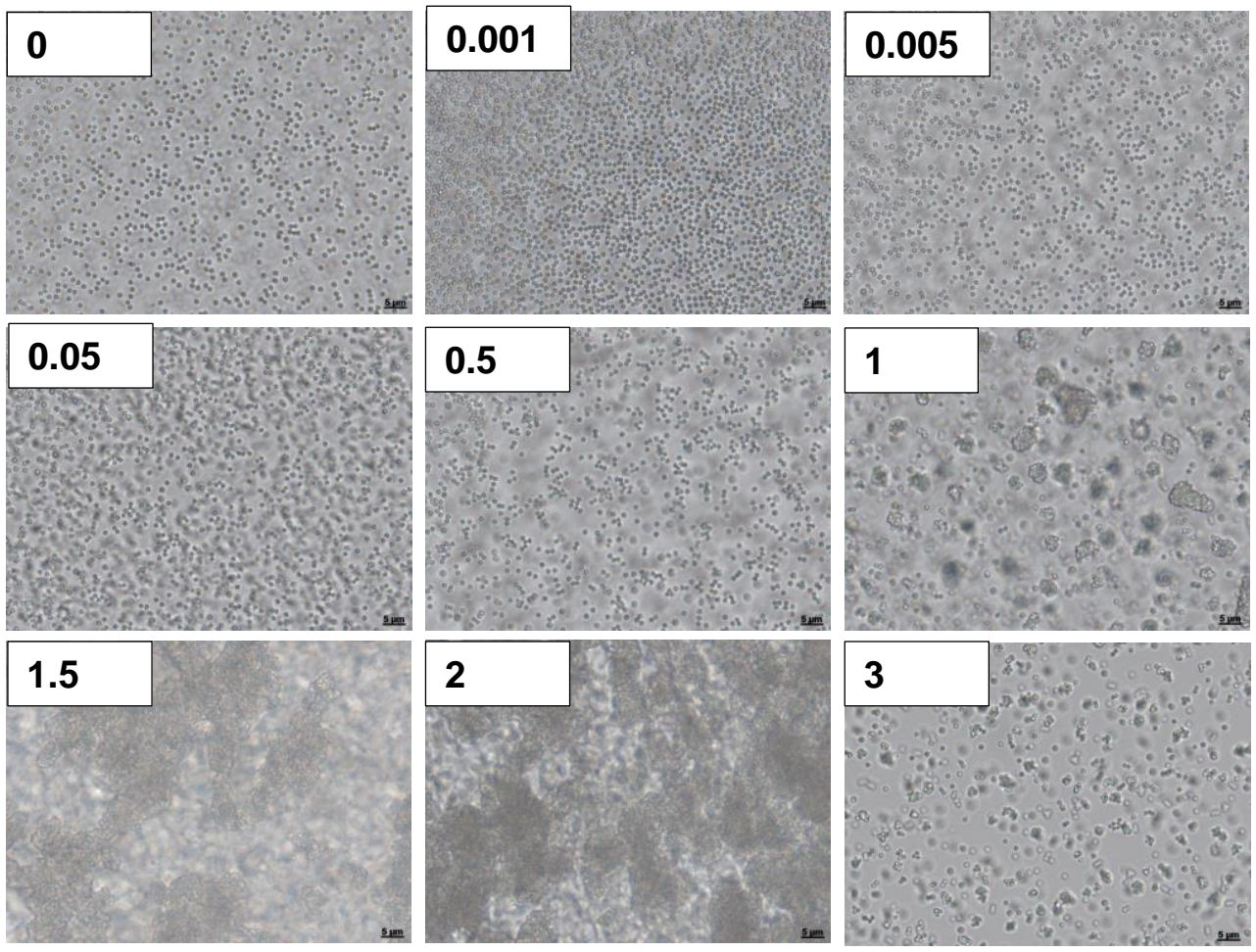

Figure 8 Microscopy images of the bottom layer of the dispersions at $20^{\circ} \mathrm{C}$ after 7 days of sample preparation. The particles are coated with lysozyme and their diameter is $862 \mathrm{~nm}$. The fibril concentrations are 0 (no fibrils added), 0.001, 0.005, 0.05, 0.5, 1, 1.5, 2 and 3 wt\%. The fibril concentration (wt\%) is indicated in the images. The scale bar corresponds to $5 \mu \mathrm{m}$.

Figure 8 shows the microscopy images of dispersions containing protein fibrils and lysozyme-coated latex particles with a diamter of $862 \mathrm{~nm}$. At low fibril concentrations (< $0.5 \mathrm{wt} \%)$ no aggregates were observed. Above $0.5 \mathrm{wt} \%$ the dispersions showed similar behaviour as the dispersions with a particle diameter of $862 \mathrm{~nm}$ as shown in Figure 7. At higher fibril concentration ( $3 \mathrm{wt} \%$ ), the dispersions are more stable than the dispersion 
without fibrils. In addition, we observed again that small aggregates are present together with some single particles.

\subsubsection{DWS}

The intensity autocorrelation functions of the dispersions were measured directly after mixing. The mean square displacement (MSD), half-life time $\left(\tau_{1 / 2}\right)$ and $p$ values are determined.
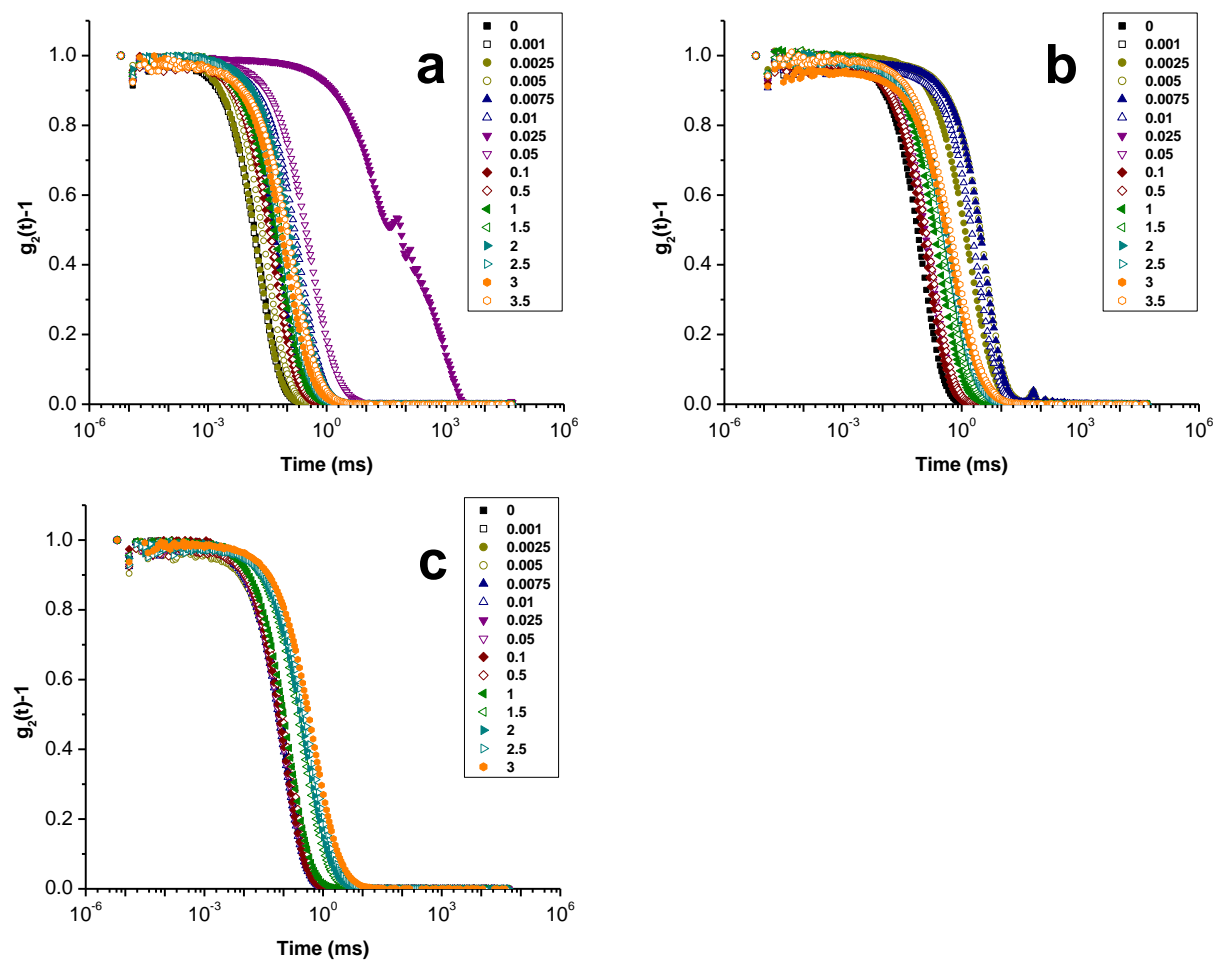

Figure 9 The normalized g2(t)-1 of dispersions containing 1 wt\% latex particles and fibril concentration of 0 (no fibrils added), $0.001,0.0025,0.005,0.0075,0.01,0.025,0.05,0.1,0.5,1,1.5$, 2, 2.5, 3 or 3.5 wt\%. (a): dispersions containing particles with a diameter of $208 \mathrm{~nm}$; (b): dispersions containing particles with a diameter of $862 \mathrm{~nm}$; (c): dispersions containing lysozymecoated particles with a diameter of $862 \mathrm{~nm}$, the sample with $3.5 \mathrm{wt} \%$ fibrils is not included. 
The intensity autocorrelation functions reflect the diffusivity of the particles. In the dispersions with a particle diameter of $208 \mathrm{~nm}$ (cf. Figure 9 a), at low fibril concentrations ( $\leq 0.0025$ wt\%), the intensity autocorrelation function is comparable to that of the dispersion without fibrils. Upon increasing fibril concentration, $\mathrm{g}_{2}(\mathrm{t})-1$ shifts to larger decorrelation times caused by a lower particle diffusivity due to aggregation, as also observed in microscopy images. At 0.025 wt\% the dispersion shows extensive aggregation (cf. Figure 6), which results in a shift to a much larger decorrelation time corresponding to a low diffusivity. Further increasing the fibril concentration up to $1 \mathrm{wt} \%$, the $\mathrm{g}_{2}(\mathrm{t})-1$ shifts back to lower decorrelation times or higher diffusivities. These decorrelation times are, however, still larger than dispersion without fibrils, which is caused by the viscosity increase of the continuous phase. Between $1.5 \mathrm{wt} \%$ and $2 \mathrm{wt} \%$ fibril concentration, due to particle aggregation, the decay of $g_{2}(t)-1$ is still somewhat slower than at 3 wt\%. Upon increasing fibril concentrations, the $\mathrm{g}_{2}(\mathrm{t})-1$ shift to larger decorrelation times with increase of the fibril concentration, presumably due to the viscosity increase of continuous phase. In line with the microscopic observations, the dispersions with a particle diameter of 862 $\mathrm{nm}$ show a similar behaviour as the dispersions with a particle diameter of $208 \mathrm{~nm}$. The main differences are the fibril concentrations at which the change in decorrelation times occur.

In lysozyme-coated particle dispersions (particle diameter of $862 \mathrm{~nm}$ ), we observed that dispersions with fibril concentration below 1 wt\% showed similar or slightly larger decorrelation times in comparison to the dispersion without fibrils. This is in line with the microscopic images, where no particle aggregation was observed (cf. Figure 8). Upon increasing fibril concentration, the dispersions showed a sharp shift to larger decorrelation times due to particle aggregation as observed by microscopy. At fibril concentration of 3 $w t \%$, where less and smaller particle aggregates were observed, the $g_{2}(t)-1$ still slightly shifts to larger decorrelation times, presumably due to the viscosity increase of the continuous phase. 

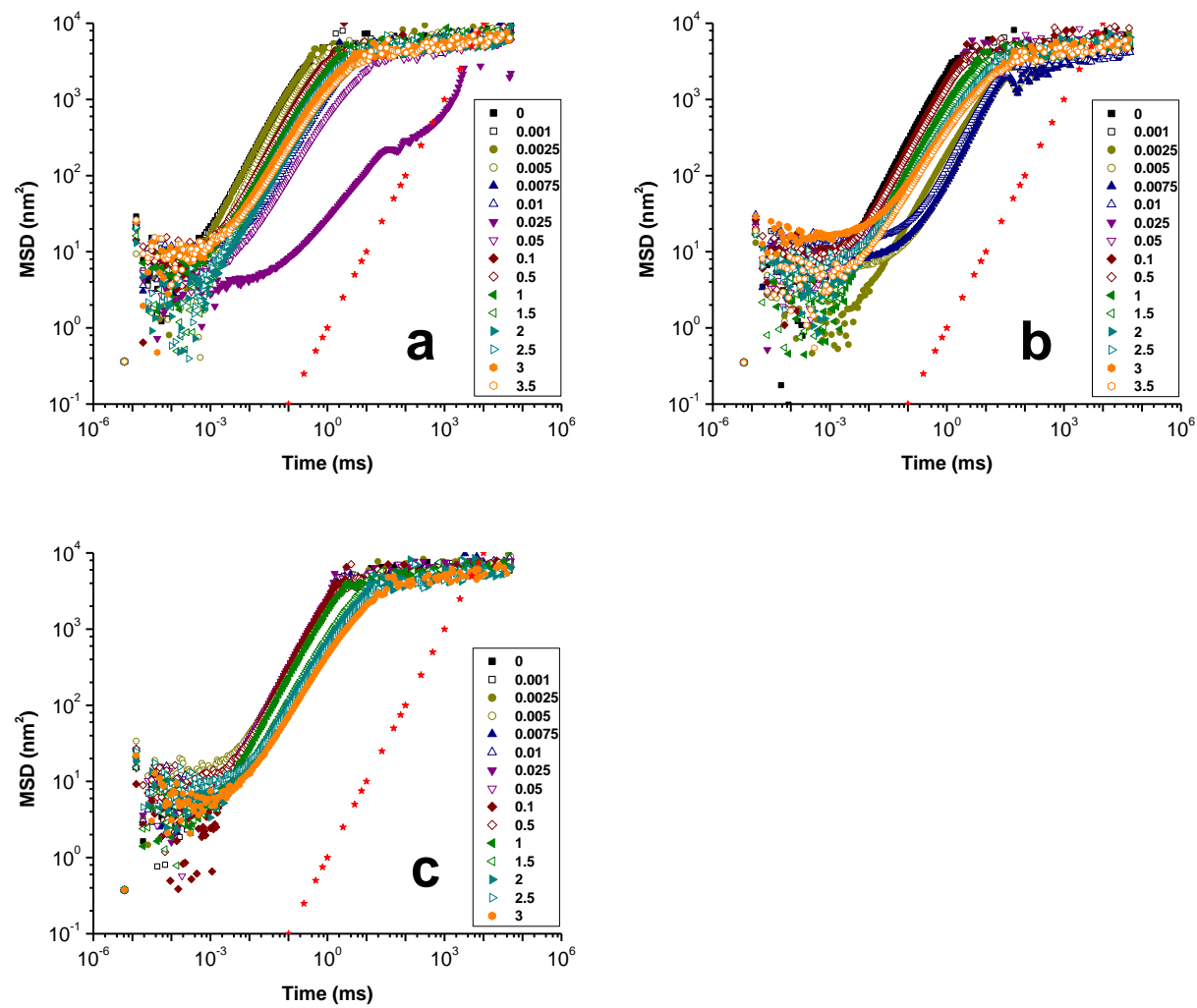

Figure 10 The mean square displacement of dispersions containing $1 \mathrm{wt} \%$ particles and fibril concentration of 0 (no fibrils added), $0.001,0.0025,0.005,0.0075,0.01,0.025,0.05,0.1,0.5,1,1.5$, 2, 2.5, 3 or 3.5 wt\%. (a): dispersions containing particles with a diameter of $208 \mathrm{~nm}$; (b): dispersions containing particles with a diameter of $862 \mathrm{~nm}$; (c): dispersions containing lysozymecoated particles with a diameter of $862 \mathrm{~nm}$, the sample with $3.5 \mathrm{wt} \%$ fibrils is not included. The line indicated by the red stars represents MSD $\sim D t^{p}$ with $p=1$ and serves as a guide to the eye. 


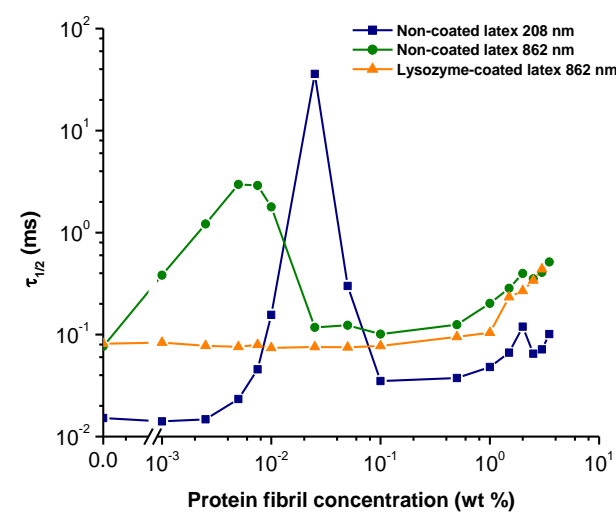

a

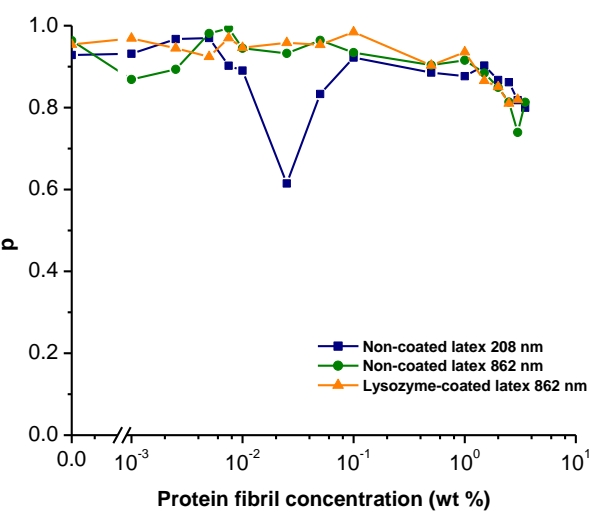

b

Figure 11 The half-life time $\tau 1 / 2$ (a) and $p$ value (b) as a function of protein fibril concentration (wt\%) for all dispersions. Dispersions containing particles with a diameter of $208 \mathrm{~nm}$ are shown in filled square. Dispersions containing particles with a diameter of $862 \mathrm{~nm}$ are shown in filled sphere. Dispersions containing lysozyme-coated particles with a diameter of $862 \mathrm{~nm}$ are shown in filled triangle. Lines are shown to guide the eye.

The half-life time $\tau_{1 / 2}$ of the dispersion containing particles with a diameter of $208 \mathrm{~nm}$ (cf. Figure $11 \mathrm{a}$ ) is constant for fibril concentrations below 0.0025 wt\%. Above this fibril concentration $\tau_{1 / 2}$ grows with increasing fibril concentration up to $0.025 \mathrm{wt} \%$. Subsequently $\tau_{1 / 2}$ decreases with increasing fibril concentration, up to $0.1 \mathrm{wt} \%$. Then, $\tau_{1 / 2}$ remains constant between fibril concentrations of 0.1 wt $\%$ and 0.5 wt\%. Further increasing the fibril concentration, leads to a sudden change in slope of the curve of $\tau_{1 / 2}$ around 2.5 wt\%. The half-life time $\tau_{1 / 2}$ of the dispersion containing particles with a diameter of $862 \mathrm{~nm}$ shows a similar pattern, with different fibril concentrations for various transitions. The half-life time $\tau_{1 / 2}$ of the dispersion containing lysozyme-coated particles slightly increases up to a fibril concentration of $0.1 \mathrm{wt} \%$. Above this concentration, the behaviour of $\tau_{1 / 2}$ is comparable to that of the other dispersions. From the MSD curves (cf. Figure 10), we obtained the $p$-values as shown in Figure $11 \mathrm{~b}$. The $p$-value in all dispersion is higher than 0.5 , indicating that the matrix behaves like a viscous medium. 

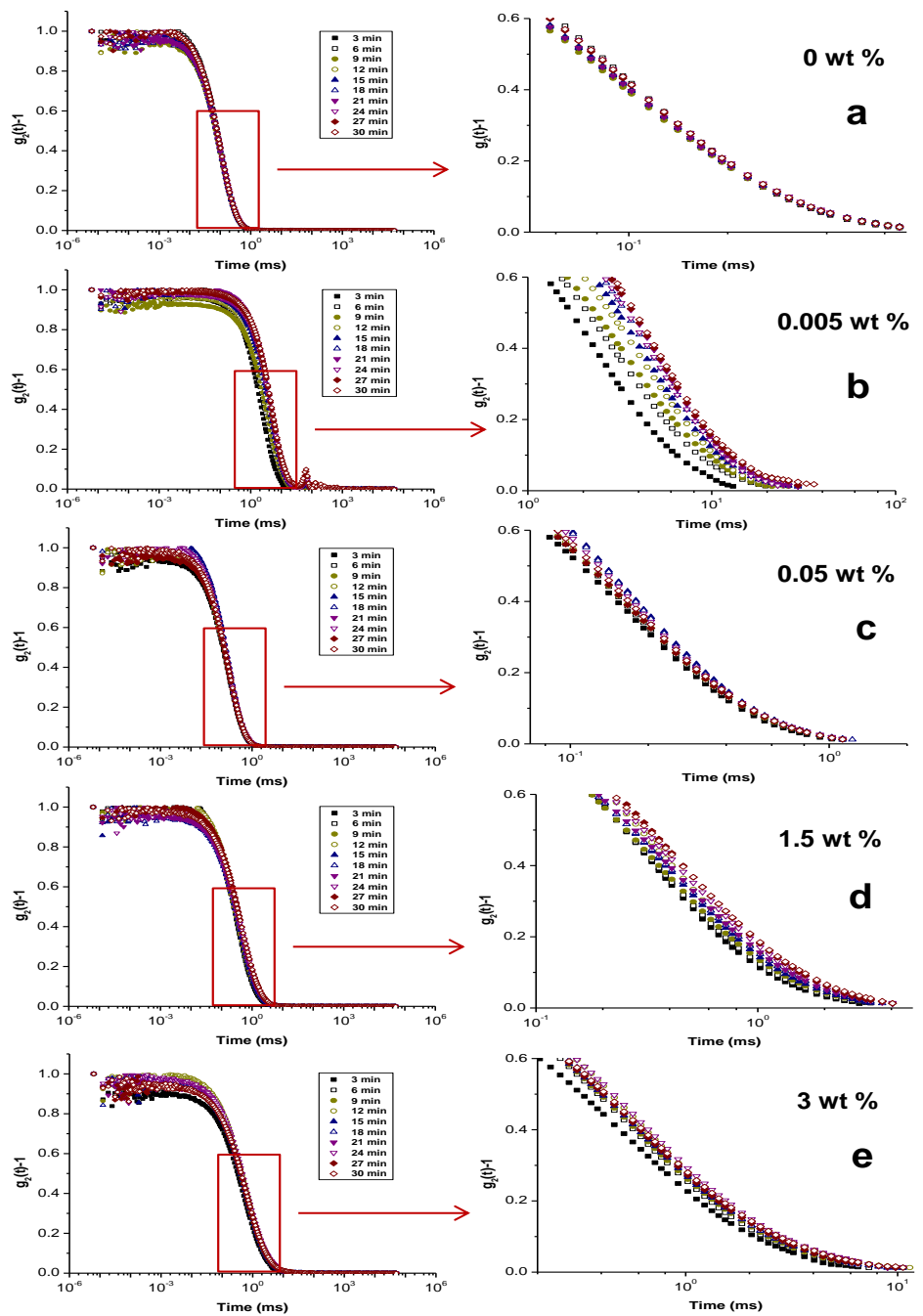

Figure 12 The normalized g2(t)-1 of the 10 measurements for dispersions after sample preparation within 30 minutes (left) and the corresponding enlargement (right) showing more detail. Dispersion was monitored immediately after preparation. Each measurement takes 3 minutes and data were collected for $\mathbf{3 0}$ minutes. The results shown above are from dispersions containing particles with a diameter of $862 \mathrm{~nm}$. (a): stable dispersion with $0 \mathrm{wt} \%$ fibrils. (b): dispersion destabilized by bridging with 0.005 wt\% fibrils. (c): stable dispersion with 0.05 wt\% fibrils. (d): dispersion destabilized by depletion with 1.5 wt\% fibrils. (e): stable dispersion with 3 wt\% fibrils. 
By monitoring the dispersions continuously for 30 minutes directly after sample preparation, we observed that for the dispersions that are stable for at least 5 days, the autocorrelation function $\mathrm{g}_{2}(\mathrm{t})-1$ did not change within this time ( $\mathrm{cf}$. Figure $12 \mathrm{a}$ and $\mathrm{c}$ ). In the dispersions that were unstable (cf. Figure $12 \mathrm{~b}$ and $\mathrm{d}$ ) we observed that the $\mathrm{g}_{2}(\mathrm{t})-1$ continuously shifted to larger decorrelation times, corresponding with the formation of particle aggregates. In the dispersions with 0.005 wt\% fibrils (cf. Figure $12 \mathrm{~b}$ ), the $\mathrm{g}_{2}(\mathrm{t})-1$ did not shift anymore after $18 \mathrm{~min}$. In the dispersion with $1.5 \mathrm{wt} \%$ of fibrils added (cf. Figure $12 \mathrm{~d}$ ) after $24 \min _{2}(\mathrm{t})-1$ did not shift anymore. These times indicate when the flocculation is completed. The larger $\tau_{1 / 2}$ for the dispersions destabilized by bridging corresponds to a larger aggregate size compared to the dispersions destabilized by depletion interaction. In dispersions that are stable (cf. Figure 12 e) with both small particle aggregates and single particles present, $\mathrm{g}_{2}(\mathrm{t})-1$ only slightly shifted to larger decorrelation times in the first 6 min, and remained the same afterwards.

\subsubsection{Discussion}

Our results showed that the stability of the dispersions is controlled by the fibril concentration, diameter and the surface charge of the particles. At low fibrils concentrations the dispersions show phase separation. In dispersions with a particle diameter of $208 \mathrm{~nm}$ and $862 \mathrm{~nm}$, both particles have the same negative surface charge ($60 \mathrm{mV}$ ), as confirmed by our zeta potential measurements. Incubating the dispersions with positively charged fibrils and subsequent washing to remove the excess of fibrils from the bulk solution led to charge reversal of the particles $(+54 \mathrm{mV})$. From this we conclude that the fibrils can adsorb onto the particles. When dispersions are mixed with low concentration of fibrils, the particles will not be completely covered, allowing bridging and destabilisation to take place as the particles and fibrils are oppositely charged. ${ }^{38,39}$ The first peak at low fibril concentration for the non-coated latex samples as shown in Figure 11 a corresponds to the flocculation as induced by bridging. In the case of particles with a diameter of $862 \mathrm{~nm}$, the flocculation due to bridging occurred at a fibril concentration lower than for the particles with a diameter of $208 \mathrm{~nm}$ (cf. Figure 11 a). This is likely related to the fact that larger particles have a lower number concentration than smaller particles. With increasing fibril concentration, the particles become fully covered, leading to (steric and/or electrostatic) stabilisation of the dispersion. ${ }^{40,41}$ This stabilization occurred at a four-fold lower fibril concentration for the larger particles (cf. Figure 11 a) 
compared to the smaller particles. The reason for this is that at fixed particle concentration (i.e., 1 wt\%) the specific area of the smaller particles is $\sim 4$ times larger compared to the larger particles and the concentration of fibrils needed to cover the surface of the particles is $\sim 4$ times higher.

Further increase of the fibril concentration leads to depletion flocculation, ${ }^{13}$ as induced by the non-adsorbed fibrils in the dispersions. Depletion flocculation induced by protein fibrils has also been described in emulsions. ${ }^{25}$ The onset of destabilization takes place at lower fibril concentrations when the particles are larger. This is in line with the volume fraction $\phi_{r}$ of rods needed to induce phase separation of colloidal spheres scales as $\phi_{r} \sim$ $D^{2} / L d$, where $d$ is the diameter of spheres, $D$ the diameter and $L$ the length of the fibrils. ${ }^{22}$

At even higher fibrils concentration, we observe again stable dispersions. We note that these dispersions are completely stable against sedimentation (at least for a time period of 14 days). This is in contrast to the dispersions without fibrils, where sedimentation takes place within 14 days. Interestingly, from microscopy images it is found that these stable dispersions contain both single particles and clusters of particles. These clusters are smaller than the aggregates in dispersions that are destabilised by either depletion or bridging interactions.

The dispersions containing lysozyme-coated particles were stable at low fibril concentrations. The lysozyme-coated particles and protein fibrils are both positively charged, excluding the possibility of bridging, and, instead, leading to electrostatic and/or steric stabilization of the dispersion. When the non-adsorbing fibrils in the dispersion exceed to a certain concentration, the dispersion is destabilized by depletion flocculation. After increasing the fibril concentration the dispersion is stabilized again, with the same characteristics as for the other two dispersions.

In all three dispersions we find stabilization at high enough fibril concentration. From Figure 4 and Figure 5 we conclude that temperature did not have a significant influence on the macroscopic stability of the dispersions. It was observed that small particle clusters are present together with single particles. All these stable dispersion also showed a better stability compared to the dispersion without fibrils. There are several mechanisms conceivable that lead to this re-stabilization. 
For instance, the increase of the bulk viscosity by the addition of fibrils ${ }^{25}$ or the formation of a particle gel stabilizing the dispersions. ${ }^{42}$ However, our DWS results (cf. Figure 11 a) have shown that the increase in bulk viscosity was only small relative to the unstable dispersions. Therefore, the stabilization of dispersions is not likely to be explained only by a viscosity increase. In addition, Figure $11 \mathrm{~b}$ showed that all dispersions have $p>0.5$, implying that $\mathrm{G}^{\prime}<\mathrm{G}^{\prime \prime},{ }^{30}$ i.e. excluding the possible formation of a particle gel. In addition, the decay of $g_{2}(t)-1$ to zero also indicates that particles can still diffuse unhindered.

DWS measurements were used to monitor the dispersions during 30 minutes directly after preparation (cf. Figure 12). In this way information on the kinetics of destabilisation can be obtained. For dispersions without fibrils the decorrelation curves overlap for different times after preparation, implying that the dispersion is stable over the time of $\mathbf{3 0}$ minutes (cf. Figure 12 a). For dispersions destabilised by bridging (cf. Figure $12 \mathrm{~b}$ ), we find that the curves shift to larger decorrelation time, and overlap with one another after 18 minutes. This indicates that aggregate formation is completed after 18 minutes. For dispersions stabilised by steric and/or electrostatic interactions the decorrelation curves all overlap (cf. Figure $12 \mathrm{c}$ ), and are identical to the curves for dispersions without fibrils. For dispersions destabilised by depletion interactions (cf. Figure $12 \mathrm{~d}$ ) the curves shift to larger decorrelation times and overlap after 24 minutes, indicating the end of aggregate formation. For dispersions stabilised at the highest fibril concentrations (cf. Figure 12 e) also show a shift to larger decorrelation times and overlap after 6 minutes. We stress that during preparation of the dispersions care was taken to disperse the latex particles into single particles by means of rigorous agitation. We conclude that directly after preparation the particles aggregate in small clusters that are stable against further aggregation. From microscopy we know that even after 7 days we still have small clusters and single particles present. The fact that single particles do exist over a period of 7 days and that aggregation takes place directly after sample preparation suggests that there is a dynamic exchange of particles in clusters with single particles in the fibril solution. The maximum cluster size suggests that there is a kinetic barrier against further growth. The kinetic barrier originates from the presence of fibrils, and could be related to the increase of osmotic pressure between two approaching clusters, as noted before. ${ }^{43}$ Moreover, the particles/clusters could be trapped in cages, as created by the surrounding fibrils. However, the typical size of these cages must be large enough to allow the auto-correlation function to decay to 
zero. This suggests that the particles can still diffuse over limited distances. This limitation in their diffusive motion could explain the absence of sedimentation.

Many researchers have observed the stabilization of colloidal dispersions at polymer concentration higher than the polymer concentration where the depletion flocculation occurred. $^{44-50}$ This stabilisation is sometimes referred to as depletion stabilisation. Interestingly, in all these works the formation of small particle clusters also was observed. The explanation of the depletion stabilization is still under discussion. The discussion mainly differentiates in thermodynamic ${ }^{48-59}$ versus kinetic ${ }^{21,43,45,47,60-64}$ arguments. In another study ${ }^{46,65}$ a different mechanism was proposed, where the stabilization was attributed to the slowdown of particle movement at high polymer concentration. In the case of colloidal dispersions containing rod-like particles, Mao and Lekkerkerker studied the depletion stabilization induced by the rods. ${ }^{21,64}$ They attribute the stabilization to a kinetic effect. We note that our systems are different from what has been studied above, since the average length of the rod-like fibrils is larger than the diameter of the particles. However, our results from microscopy and DWS also point in the direction of kinetic stabilisation, i.e. depletion stabilization.

\subsection{Conclusion}

We find that dispersions containing particles and oppositely charged fibrils show 4 different stability regimes as a function of fibril concentration. With increasing fibril concentration we find: 1 ) bridging flocculation; 2) steric and/ or electrostatic stabilization; 3) depletion flocculation; and 4) depletion stabilization. Interestingly, the depletion stabilized dispersions contain single particles together with small clusters that cannot grow beyond a certain size. Although the depletion stabilization mechanism still needs more research, our results point to a mechanism based on a kinetic barrier, which depends on fibril concentration.

\subsection{Acknowledgements}

This research is financially supported by NanoNextNL (a consortium of Dutch government and 130 other partners). We thank Harry Baptist for TEM image analysis and Tiny Franssen-Verheijen for SEM image analysis. 


\subsection{References}

1. C. M. Dobson, Nature, 2003, 426, 884-890.

2. M. R. H. Krebs, G. L. Devlin and A. M. Donald, Biophysical Journal, 2007, 92, 1336-1342.

3. E. van der Linden and P. Venema, Current Opinion in Colloid \& Interface Science, 2007, 12, 158165.

4. P. Aymard, T. Nicolai, D. Durand and A. Clark, Macromolecules, 1999, 32, 2542-2552.

5. G. M. Kavanagh, A. H. Clark and S. B. Ross-Murphy, International Journal of Biological Macromolecules, 2000, 28, 41-50.

6. C. Akkermans, P. Venema, A. J. van der Goot, H. Gruppen, E. J. Bakx, R. M. Boom and E. van der Linden, Biomacromolecules, 2008, 9, 1474-1479.

7. E. H. C. Bromley, M. R. H. Krebs and A. M. Donald, Faraday Discussions, 2005, 128, 13-27.

8. C. Akkermans, A. J. Van der Goot, P. Venema, E. Van der Linden and R. M. Boom, International dairy journal, 2008, 18, 1034-1042.

9. C. Veerman, L. M. C. Sagis and E. van der Linden, Macromolecular Bioscience, 2003, 3, 243-247.

10. L. M. C. Sagis, R. de Ruiter, F. J. R. Miranda, J. de Ruiter, K. Schroën, A. C. van Aelst, H. Kieft, R. Boom and $\mathrm{E}$. van der Linden, Langmuir, 2008, 24, 1608-1612.

11. K. N. P. Humblet-Hua, G. Scheltens, E. van der Linden and L. M. C. Sagis, Food Hydrocolloids, 2011, 25, 569-576.

12. N. P. K. Humblet-Hua, E. van der Linden and L. M. C. Sagis, Journal of Agricultural and Food Chemistry, 2012, 60, 9502-9511.

13. H. N. W. Lekkerkerker and R. Tuinier, Colloids and the depletion interaction, Springer, Dordrecht ; New York, 2011.

14. G. A. Vliegenthart, A. van Blaaderen and H. N. W. Lekkerkerker, Faraday Discussions, 1999, 112, 173-181.

15. M. Adams, Z. Dogic, S. L. Keller and S. Fraden, Nature, 1998, 393, 349-352.

16. Y. L. Chen and K. S. Schweizer, Journal of Chemical Physics, 2002, 117, 1351-1362.

17. D. Guu, J. Dhont, G. Vliegenthart and M. Lettinga, Journal of Physics: Condensed Matter, 2012, 24, 464101.

18. G. H. Koenderink, G. A. Vliegenthart, S. G. J. M. Kluijtmans, A. van Blaaderen, A. P. Philipse and H. N. W. Lekkerkerker, Langmuir, 1999, 15, 4693-4696.

19. W. H. Li, T. Yang and H. R. Ma, Journal of Chemical Physics, 2008, 128, 044910.

20. K. H. Lin, J. C. Crocker, A. C. Zeri and A. G. Yodh, Physical Review Letters, 2001, 87, 088301.

21. Y. Mao, M. E. Cates and H. N. W. Lekkerkerker, Physical Review Letters, 1995, 75, 4548-4551.

22. G. A. Vliegenthart and H. N. W. Lekkerkerker, Journal of Chemical Physics, 1999, 111, 41534157.

23. K. Yaman and et al., EPL (Europhysics Letters), 1998, 42, 221. 
24. N. Yasarawan and J. S. van Duijneveldt, Soft Matter, 2010, 6, 353-362.

25. T. B. J. Blijdenstein, C. Veerman and E. van der Linden, Langmuir, 2004, 20, 4881-4884.

26. A. Kroes-Nijboer, P. Venema, H. Baptist and E. van der Linden, Langmuir, 2010, 26, 1309713101.

27. C. O. Klein van, P. Venema, L. M. C. Sagis, D. v. Dusschoten, M. Wilhelm, H. W. Spiess, E. v. d. Linden, S. S. Rogers and A. M. Donald, Applied rheology, 2007, 17, 45210.

28. S. S. Rogers, P. Venema, L. M. C. Sagis, E. van der Linden and A. M. Donald, Macromolecules, 2005, 38, 2948-2958.

29. H. M. Wyss, S. Romer, F. Scheffold, P. Schurtenberger and L. J. Gauckler, Journal of Colloid and Interface Science, 2001, 241, 89-97.

30. T. G. Mason, Rheologica Acta, 2000, 39, 371-378.

31. A. J. Breugem, F. Bouchama and G. J. M. Koper, Surface Coatings International Part B: Coatings Transactions, 2005, 88, 135-138.

32. S. G. Bolder, H. Hendrickx, L. M. C. Sagis and E. van der Linden, Journal of Agricultural and Food Chemistry, 2006, 54, 4229-4234.

33. S. G. Bolder, International dairy journal, 2007, 17, 846-853.

34. L. N. Arnaudov, R. de Vries, H. Ippel and C. P. M. van Mierlo, Biomacromolecules, 2003, 4, 1614 1622.

35. Z. G. Peng, K. Hidajat and M. S. Uddin, Colloids and Surfaces B-Biointerfaces, 2004, 35, 169-174.

36. C.-S. Chern, C.-K. Lee and C.-J. Chang, Colloid and Polymer Science, 2004, 283, 257-264.

37. C. A. Haynes, E. Sliwinsky and W. Norde, Journal of Colloid and Interface Science, 1994, 164, 394-409.

38. E. Dickinson and L. Eriksson, Advances in Colloid and Interface Science, 1991, 34, 1-29.

39. E. G. M. Pelssers, M. A. C. Stuart and G. J. Fleer, Journal of the Chemical Society, Faraday Transactions, 1990, 86, 1355-1361.

40. D. H. Napper, Journal of Colloid and Interface Science, 1977, 58, 390-407.

41. B. Vincent, Advances in Colloid and Interface Science, 1974, 4, 193-277.

42. A. Parker, P. A. Gunning, K. Ng and M. M. Robins, Food Hydrocolloids, 1995, 9, 333-342.

43. R. I. Feigin and D. H. Napper, Journal of Colloid and Interface Science, 1980, 75, 525-541.

44. P. J. Lu, J. C. Conrad, H. M. Wyss, A. B. Schofield and D. A. Weitz, Physical Review Letters, 2006, 96, 028306 .

45. A. Sharma, S. N. Tan and J. Y. Walz, Journal of Colloid and Interface Science, 1997, 190, 392-407.

46. M. Yasrebi, W. Y. Shih and I. A. Aksay, Journal of Colloid and Interface Science, 1991, 142, 357 368.

47. S. Kim, K. Hyun, J. Y. Moon, C. Clasen and K. H. Ahn, Langmuir, 2015, 31, 1892-1900.

48. C. Cowell, R. Li-In-On and B. Vincent, Journal of the Chemical Society, Faraday Transactions 1: Physical Chemistry in Condensed Phases, 1978, 74, 337-347. 
49. B. Vincent, P. Luckham and F. Waite, Journal of Colloid and Interface Science, 1980, 73, 508-521.

50. J. Clarke and B. Vincent, Journal of the Chemical Society, Faraday Transactions 1: Physical Chemistry in Condensed Phases, 1981, 77, 1831-1843.

51. J. Clarke and B. Vincent, Journal of Colloid and Interface Science, 1981, 82, 208-216.

52. B. Vincent, J. Edwards, S. Emmett and A. Jones, Colloids and Surfaces, 1986, 18, 261-281.

53. J. Scheutjens and G. Fleer, Advances in Colloid and Interface Science, 1982, 16, 361-380.

54. G. Fleer and J. Scheutjens, The stability of dispersions of hard spherical particles in the presence of nonadsorbing polymer, 1984.

55. G. Fleer and J. Scheutjens, Croatica chemica acta, 1987, 60, 477-494.

56. A. P. Gast and L. Leibler, The Journal of Physical Chemistry, 1985, 89, 3947-3949.

57. A. P. Gast and L. Leibler, Macromolecules, 1986, 19, 686-691.

58. N. Dutta and D. Green, Langmuir, 2008, 24, 5260-5269.

59. J. Groenewold and W. K. Kegel, The Journal of Physical Chemistry B, 2001, 105, 11702-11709.

60. Y.-L. Chen and K. Schweizer, The Journal of Chemical Physics, 2002, 117, 1351-1362.

61. A. Ogden and J. Lewis, Langmuir, 1996, 12, 3413-3424.

62. J. Y. Walz and A. Sharma, Journal of Colloid and Interface Science, 1994, 168, 485-496.

63. X. Zhang, M. R. Servos and J. Liu, Journal of the American Chemical Society, 2012, 134, 99109913.

64. Y. Mao, M. E. Cates and H. N. W. Lekkerkerker, Physica A: Statistical Mechanics and its Applications, 1995, 222, 10-24.

65. J. Liu, W. Y. Shih, R. Kikuchi and I. A. Aksay, Journal of Colloid and Interface Science, 1991, 142, 369-377. 
Chapter 7 


\section{Chapter 8}

\section{Tuning emulsion stability using} protein-cellulose bi-fibrillar networks

Submitted as:

Jinfeng Peng, Nina van der Hulst, Krassimir P. Velikov, Paul Venema and Erik van der Linden. Tuning emulsion stability using protein-cellulose bi-fibrillar networks. 


\section{Abstract}

We investigated the emulsion stability containing a bi-fibrillar network consisting of both whey protein isolate (WPI) fibrils and bacterial cellulose (BC) microfibrils at $\mathrm{pH} 2$. The stability of emulsions in the presence of only WPI protein fibrils has been part of a previous publication (i.e. Chapter 6 ). ${ }^{1}$ Emulsions in the presence of only BC microfibrils, are stabilized by a yield stress of the BC microfibril network. The presence of WPI fibrils may lead to depletion induced oil droplet aggregation, and enhanced creaming can be prevented when the concentration of BC microfibrils is sufficiently high. Combining two types of fibrillar structures in emulsions leads to a competition between stabilization and/or destabilization effects of the WPI fibrils and BC microfibrils. As a result, emulsion stability with different rheological properties and microstructures can be tuned by varying the concentration ratios of the two fibrillar structures. The stabilisation of emulsions by BC microfibrils and WPI-BC bi-fibrillar networks are temperature independent. 


\subsection{Introduction}

Emulsions are thermodynamically unstable systems and are present in various products such as pharmaceuticals, cosmetics and foods. Creaming, Ostwald ripening, flocculation and droplet coalescence are phenomena that lead to emulsion instability. ${ }^{2,3}$ To confer long-term emulsion stability, stabilizers such as proteins and/or polysaccharides are normally added to emulsions. ${ }^{2-4}$ Stabilizing mechanisms are for instance, rheological properties of the continuous phase, ${ }^{4}$ formation of emulsion gel network, ${ }^{5}$ and so-called "depletion stabilization", ${ }^{1,6}$ which is less commonly known. Apart from stabilization of emulations, the addition of protein and/or polysaccharides may also induce destabilization of the emulsions under certain conditions. For instance, a destabilization mechanism may be droplet aggregation via depletion flocculation. ${ }^{7,8}$

Protein fibrils are rod-like aggregates formed from proteins when certain conditions are applied. ${ }^{9-11}$ Many proteins have the ability to form fibrils, such as whey protein, ${ }^{12}$ soy protein, ${ }^{13}$ pea protein, ${ }^{14}$ ovalbumin, ${ }^{15}$ and lysozyme. ${ }^{16}$ Among them, protein fibrils formed from whey protein, in particular, $\beta$-lactoglobulin $(\beta-\mathrm{lg})$, has received considerable attention regarding its formation mechanism and physical properties. Upon heating at low $\mathrm{pH}$ and low ionic strength, the peptides from protein hydrolysis will assemble into fibrils. ${ }^{17}$ ${ }^{18}$ The fibrils formed are semi-flexible and have a high aspect ratio, due to its diameter in the order of nanometer, while the length is in the order of micrometers. ${ }^{19}$ Protein fibrils have been reported to be interesting candidates as functional ingredients in foods. ${ }^{20}$ Recently, the protein fibrils were found to induce complex phase behaviour in colloidal dispersions. ${ }^{1}$ 21, 22 More importantly, the fibrils are found to stabilize emulsions by depletion stabilization, while keeping the emulsions as a low viscosity fluid. ${ }^{1,21}$ Depletion flocculation induced by protein fibrils in emulsion has also been reported earlier. ${ }^{22}$

Bacterial cellulose $(B C)$ is a microbial polysaccharide, which has received considerable attention in literature due to its unique properties and broad applications in industry. In foods, it is recognized as an important functional ingredient due to its purity and potential use as a thickening, gelling, stabilizing and water binding agent. ${ }^{23-29}$ Moreover, BC offers health benefits as a dietary fibre and low caloric ingredient. ${ }^{30-32}$ The popular dessert in Philippines, Nata de Coco, mainly consists of $\mathrm{BC}$ and water. ${ }^{33} \mathrm{~A}$ recent study applied highenergy mechanical de-agglomeration to process the Nata de Coco to obtain a colloidal dispersion consisting of $\mathrm{BC}$ microfibrils. ${ }^{34}$ Microfibrils in $\mathrm{BC}$ are in the form of a ribbon- 
like structure, with a width of nanometers and a length of micrometers. The hydroxyl groups mediated hydrogen bonding lead to agglomeration of the microfibrils, forming a space-filling network. Above a certain BC concentration ( $0.1 \mathrm{wt} \%$ ), the microfibrils form a self-supporting gel. ${ }^{34}$ The viscosity of the dispersions increase substantially with increasing $B C$ concentrations. The potential of $B C$ microfibrils as an emulsifier and stabilizer in emulsions has also been studied before. ${ }^{26,28,29}$ In the meanwhile, BC has been utilized as a stabilizing agent ${ }^{35}$ to retain the shape of ice cream and as a heat-stable suspending agent ${ }^{25}$ to prevent cacao particles in a chocolate drink from sedimentation. The stabilizing effect of $\mathrm{BC}$ is mainly attributed to the rheological properties of its network. ${ }^{23-25,29,35}$

In Chapter 3 it was found that WPI fibrils and BC microfibrils do not phase separate. In this Chapter, we aim to investigate the functionality of mixed WPI fibrils and BC microfibrils on emulsion stability.

\subsection{Material and methods}

\subsubsection{Whey protein isolate (WPI) solution}

WPI powder ((BiPRO, Davisco, USA) was dispersed in an $\mathrm{HCl}$ solution of $\mathrm{pH} 2$ to prepare a stock solution of approximately $7.5 \% \mathrm{WPI}$. After being stirred at $4{ }^{\circ} \mathrm{C}$ overnight, the $\mathrm{pH}$ was adjusted to $\mathrm{pH} 2$ using a $6 \mathrm{M} \mathrm{HCl}$ solution. Subsequently, the solution was filtered using a filter (Hydrophilic PES $0.45 \mu \mathrm{m}$, Millipore Millex-HP) for removal of the undissolved proteins. The protein concentration of the solution was measured using a UV spectrophotometer (Cary 50 Bio, Varian) at a wavelength of $278 \mathrm{~nm}$ in combination of a calibration curve determined from WPI solutions at known concentrations. The solution was stored at $4{ }^{\circ} \mathrm{C}$ for further use in fibril formation and emulsion preparation.

\subsubsection{WPI fibril formation}

A 4 wt\% WPI solution was prepared by diluting the WPI stock solution with an $\mathrm{HCl}$ solution at $\mathrm{pH}$ 2. The solution was heated and stirred in a heating block (RT 15 Power, IKA-WERKE) at $80{ }^{\circ} \mathrm{C}$ for $22 \mathrm{~h}$ to form protein fibrils. After heating, the fibril solution was cooled down in ice-water and then stored at $4^{\circ} \mathrm{C}$ for further use. The protein fibril concentration (wt\%) mentioned in this paper refers to the WPI concentration before heating. 


\subsubsection{Bacterial cellulose $(\mathrm{BC})$ microfibril dispersion}

The BC microfibril dispersion with a concentration of 1 wt\% was kindly supplied by Unilever R\&D Vlaardingen, the Netherlands. The preparation method is the same as described in Chapter 3. The detailed procedure for preparing the dispersion can be found elsewhere. $^{34,36}$

\subsubsection{Oil-in-water (O/W) emulsion}

To prepare a $40 \%(\mathrm{v} / \mathrm{v})$ o/w emulsion, WPI was used as an emulsifier. $1 \mathrm{wt} \% \mathrm{WPI}$ was prepared from dilution of the WPI stock solution. For emulsion preparation, sunflower oil (Reddy, The Netherlands, obtained from a local supermarket) was first gradually added into the $1 \mathrm{wt} \%$ WPI solution with continues mixing using a high-speed blender (Ultra Turrax, T25 digital, IKA) at a speed of $9000 \mathrm{rpm}$ for $5 \mathrm{~min}$. After pre-mixing, the sample was passed five times through a homogenizer (Labscope, Delta instruments, The Netherlands) at a pressure of 70 bar. The emulsion was kept at $4{ }^{\circ} \mathrm{C}$ and used for sample preparation within $12 \mathrm{~h}$ after storage.

\subsubsection{Sample preparation}

To investigate the effect of WPI-BC bi-fibrillar networks on emulsion stability, three systems were prepared as follows:

(1) BC microfibrils systems: only $B C$ microfibrils were added to the emulsions. A range of $B C$ microfibril concentrations were diluted from the stock dispersion using an $\mathrm{HCl}$ solution of $\mathrm{pH} 2$ and used in emulsions. The final concentrations of $\mathrm{BC}$ microfibrils in the emulsion are: $0,0.001,0.0025,0.005,0.0075,0.01,0.025,0.05,0.1,0.15,0.2$, $0.3,0.35,0.4,0.45,0.5$, and $0.6 w t \%$.

(2) WPI systems: only WPI fibrils were added to the emulsions. The final concentrations of the protein fibrils in the emulsions are: $0,0.01,0.05,0.1,0.5,1,2$ and $2.5 \mathrm{wt} \%$.

(3) WPI-BC bi-fibrillar systems: the mixture of WPI fibrils and BC microfibrils were added to the emulsions. The concentrations of WPI fibrils used in the mixture are: $0,0.01$, $0.05,0.1,0.5,1,2$, and 2.5 wt\%. The BC concentrations used in the mixtures are 0 , $0.005,0.05,0.1,0.15,0.2,0.3$, and 0.35 wt\%. The WPI fibrils and BC microfibrils were prepared separately, then mixed together, and subsequently mixed with the emulsions. 
In all systems, the final volume fraction of the oil droplets was fixed to $10 \%(\mathrm{v} / \mathrm{v})$. All dilutions are prepared using an $\mathrm{HCl}$ solution at $\mathrm{pH}$ 2. The samples are mixed by adding emulsions to pure BC microfibrils, pure WPI fibrils or WPI-BC bi-fibrillar mixtures. Samples are mixed using a vortex for $15 \mathrm{~s}$.

\subsubsection{Morphology of WPI fibrils and BC microfibrils}

Transmission electron microscopy (TEM) was used to image the WPI fibrils and BC microfibrils. Dilution was performed to reach a final concentration of $0.02 \mathrm{wt} \%$ for WPI fibrils and 0.025 wt\% for $B C$ microfibrils prior to imaging. A droplet of the sample was placed on a carbon film supported by a copper grid, which has been pre-treated by glow discharge. The droplet was dried by a filter paper after $15 \mathrm{~s}$ to remove most of the water. Afterwards, a droplet of $2 \%$ uranyl acetate was added onto the grid for WPI fibrils, whereas for $\mathrm{BC}$ microfibrils, a drop of $2 \%$ phosphortungsten acid was added onto the grid. Excess liquid was removed after $15 \mathrm{~s}$ by another filter paper. Images were taken using a transmission electron microscope (JEOL JEM-1011, Tokyo, Japan for the WPI fibrils and a Tecnai 20 system from FEI for the BC microfibrils).

\subsubsection{Size distribution of oil droplets}

Multi-angle static laser light scattering instrument (Mastersizer MS2000, Malvern Instruments) was used to measure the size distribution of oil droplets. For all batches, we obtain an average diameter of the oil droplets, $D[3,2]$, of $1.8 \pm 0.2 \mu \mathrm{m}$.

\subsubsection{Characterization of emulsion stability}

\subsubsection{Visual observation}

Samples were prepared in a $2 \mathrm{ml}$ transparent bottles and photographic images were taken at time 0,1 hour, 1 day and 7 days after sample preparation at $20^{\circ} \mathrm{C}$.

\subsubsection{Rheological properties}

Both the viscosity and visco-elastic properties of the samples were measured using a stress-controlled rheometer (MCR 302, Anton Paar) equipped with sandblasted concentric cylinder geometry (CC17/TI/S-SN38492). Samples were transferred into the rheometer using a pipette with the tip that was cut off to minimise fibril alignment during sample 
transfer. A solvent trap was used to prevent evaporation. The shear viscosity was determined by increasing the shear rate from 0.01 to $5001 / \mathrm{s}$ at $20{ }^{\circ} \mathrm{C}$ in $15 \mathrm{~min}$. The viscoelastic properties of the samples were measured by performing a strain sweep at a frequency of $1 \mathrm{~Hz}$ by increasing the strain from 0.01 to $1000 \%$. All measurements were performed at $20^{\circ} \mathrm{C}$.

\subsubsection{Confocal laser scanning microscopy (CLSM)}

CLSM (Zeiss $200 \mathrm{~m}$ Axiovert, Thornwood, NY) was used to image the microstructure of the creaming layer (upper part) of the emulsions. A small drop of $0.2 \mathrm{wt} \%$ Nile blue (Merck, Germany) in Milli-Q water was added to stain the oil droplets prior to imaging. Subsequently, about $20 \mu \mathrm{L}$ emulsion was transferred to a chamber prepared from a microscope glass slide (Menzel-glaser, $50 \mathrm{~mm}$, Thermo Scientific, Germany) and a coverslip (Menzel-Glaser, $15 \mathrm{~mm} \times 15 \mathrm{~mm}$, Thermo Scientific, Germany). Images were taken by an oil immersion objective (Plan-Apochromat, 100×/1.46 oil, Zeiss, Germany). Emulsions were imaged at an excitation wavelength of $488 \mathrm{~nm}$ and an emission wavelength of $525 \mathrm{~nm}$.

\subsubsection{Effect of temperature on emulsion stability}

To investigate the effect of temperature on the stability of the above prepared emulsions (i.e., with addition BC microfibrils and/or WPI fibrils, the samples were stored at both room temperature $\left(\sim 20^{\circ} \mathrm{C}\right.$ in a dark cabinet) and $45{ }^{\circ} \mathrm{C}$ (in an oven) for 7 days. Photographic and CLSM images were taken over time to check for stability.

\subsubsection{Effect of $\mathrm{NaCl}$ concentration on emulsion stability}

To investigate the influence of salt on emulsion stability, $150 \mathrm{mM} \mathrm{NaCl}$ was added to the emulsions. Photographic and CLSM images were taken to check for stability.

\subsection{Results and discussion}

\subsubsection{Morphology of WPI fibrils and BC microfibrils}

Both WPI fibrils and BC microfibrils were characterized using TEM. As can be observed in Figure 1, the WPI fibrils are semi-flexible and polydisperse. The average length is 
approximately $2 \mu \mathrm{m}$. The diameter of the fibrils have been previously reported to be 4 $\mathrm{nm}^{37}$ under the conditions used currently. With increase in WPI fibril concentration, four different concentration regimes can be identified: dilute, semi-dilute, concentrated isotropic and nematic regime. ${ }^{20,38}$ At low ionic strength at $\mathrm{pH} 2$, the transition from isotropic to nematic regime was determined to be $\sim 0.4$ wt\% fibrils. ${ }^{20,39}$

In the case of BC microfibrils (Figure 2), flexible and long fibrillar structures can be observed, where the length of the microfibrils is too long to be measured. The $B C$ microfibrils are not present as individual fibrils, instead, they show up as bundles of fibrils. ${ }^{40}$ Previous studies reported that BC microfibrils, that were prepared in the same way, are approximately $9 \mathrm{~nm}$ thick, $60 \mathrm{~nm}$ wide and up to several micrometers long. ${ }^{34}$ The dispersion consists of fibril bundles, flocs and voids ranging from tens to hundreds of micrometres, which leads to a highly heterogeneous dispersion. Upon increasing the $B C$ concentrations, the size of flocs increases and the size of voids decreases. However, the size of the bundles remained unchanged. ${ }^{34}$
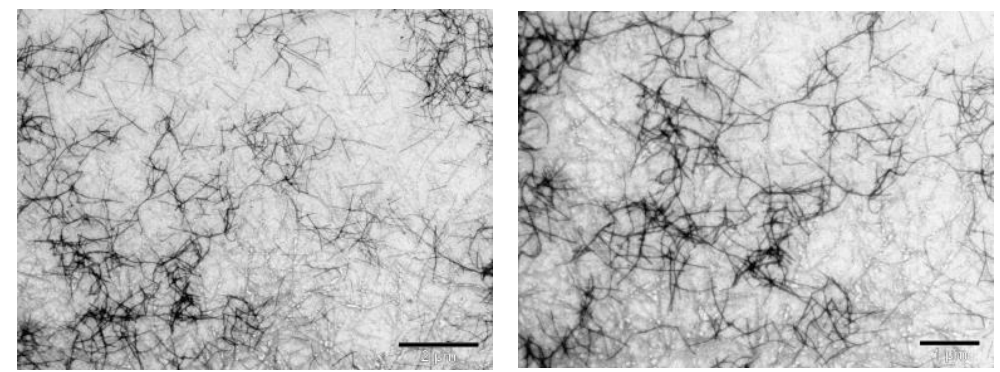

Figure 1 TEM images of 4 wt\% WPI fibrils. The scale bar corresponds to $2 \mu \mathrm{m}$ (left) and $1 \mu \mathrm{m}$ (right). 

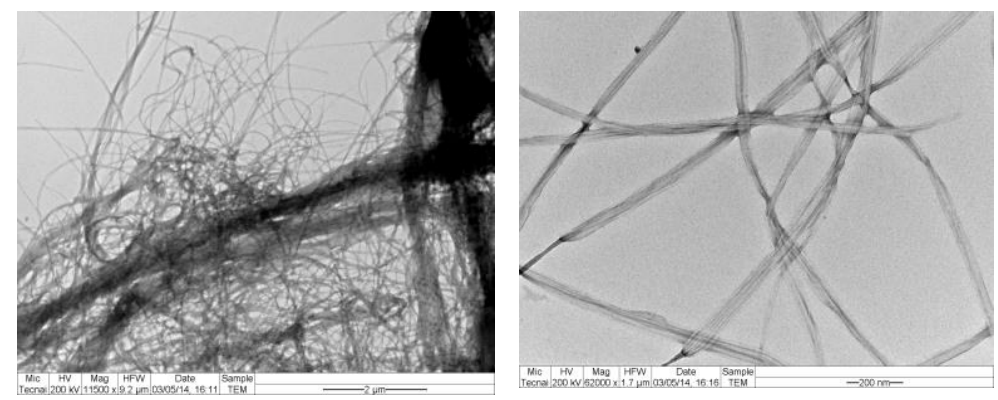

Figure 2 TEM images of 0.5 wt\% BC microfibrils. The scale bar corresponds to $2 \mu \mathrm{m}$ (left) and 200 nm (right).

\subsubsection{Visual observation}

Macroscopic images of emulsions are taken at time 0, 1 day, and 7 days after sample preparation. The results are shown in Figure 3 (for the emulsions with $B C$ microfibrils) and Figure 4 (for the emulsions containing both WPI fibrils and BC microfibrils).

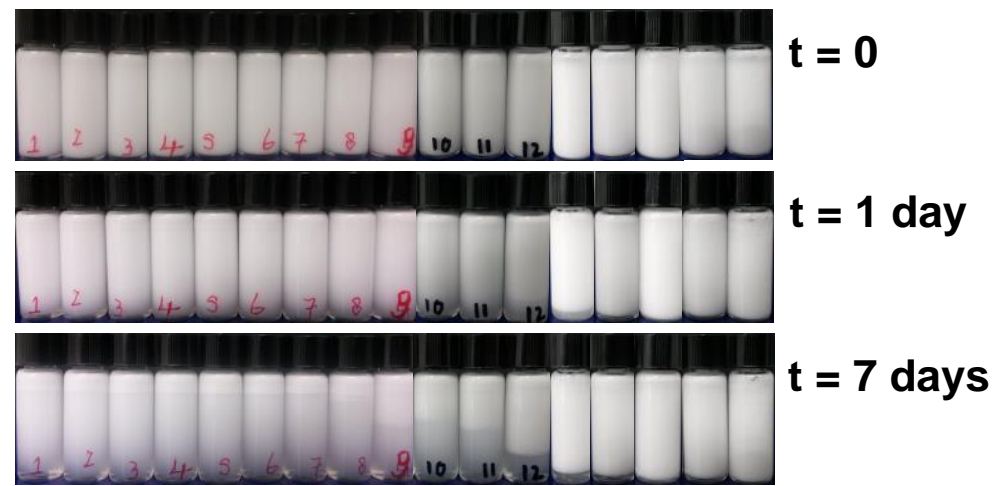

Figure 3 Visual observation at $20^{\circ} \mathrm{C}$ of $10 \%(\mathrm{v} / \mathrm{v})$ o/w emulsions containing $\mathrm{BC}$ microfibrils at concentrations (increasing from left to right) of $0,0.001,0.0025,0.005,0.0075,0.01,0.025,0.05$, $0.1,0.15,0.2,0.3,0.35,0.4,0.45,0.5$, and $0.6 \mathrm{wt} \% \mathrm{BC}$. The different rows refer to the samples at 0 , 1 day, and 7 days after preparation.

In the emulsion without addition of BC microfibril (0 wt\%), creaming due to density difference between the oil and water was observed after 7 days. In emulsions containing only $\mathrm{BC}$ microfibrils (Figure 3), we observed that up to $\mathrm{BC}$ microfibril concentration of 0.05 wt\%, the emulsions showed similar creaming behaviour to the emulsion without $B C$ 
microfibril at 7 days after sample preparation, i.e. a creaming layer was observed on the top. Upon increasing BC microfibril concentration to $0.2 \mathrm{wt} \%$, the creaming behaviour was slowed down, with an even larger extent of slowing down at $0.3 \mathrm{wt} \%$ BC microfibrils. Interestingly, the bottom layer was observed to be less turbid, with increasing $B C$ microfibril concentration, in comparison to the emulsion without $B C$ microfibrils. At $B C$ microfibril concentration of $0.35 \mathrm{wt} \%$, the creaming was less pronounced, also leaving a less turbid thin layer at the bottom of the sample. Further increasing the BC microfibril concentration ( $\geq 0.4 \mathrm{wt} \%$ ), the emulsions remained stable for 7 days. Moreover, the emulsions containing 0.5 and $0.6 \mathrm{wt} \% \mathrm{BC}$ microfibrils were found to be stable against creaming up to 14 days after sample preparation (results not shown). 


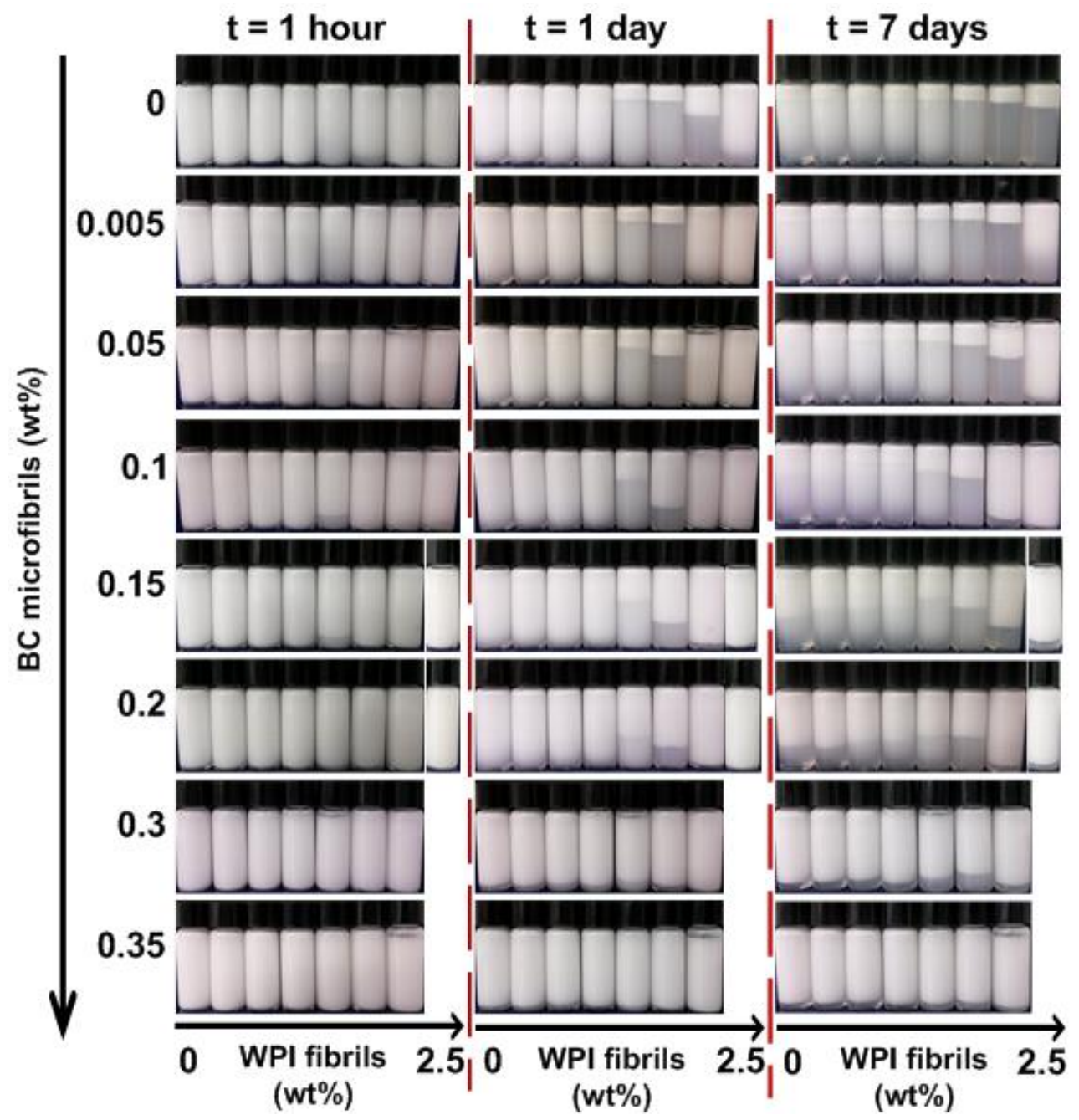

Figure 4 Visual observations at $20^{\circ} \mathrm{C}$ of $10 \%(\mathrm{v} / \mathrm{v})$ o/w emulsions containing both WPI fibrils and $B C$ microfibrils at different concentrations. The concentrations of $B C$ microfibrils are $0,0.005,0.05$, $0.1,0.15,0.2,0.3$ and $0.35 \mathrm{wt} \%$, as indicated on the left side of the image. For each BC microfibril concentration, the concentrations of WPI fibrils are $0,0.01,0.05,0.1,0.5,1,2$, and 2.5 wt\% WPI fibrils (as indicated below the images with concentration increasing from 0 to $2.5 \mathrm{wt} \%$ from left to right). In emulsions containing 0.3 and 0.35 wt\% BC, the WPI fibril at 2.5 wt\% are not included as the WPI fibril concentration in the stock solution (4 wt\%) is too low for dilution to $2.5 \mathrm{wt} \%$. The different columns refer to the samples at 1 hour, 1 day, and 7 days after preparation. 
In emulsions containing only WPI fibrils, as shown on the top row in Figure 4, the behaviour was in agreement with previous studieson these systems. ${ }^{1,22}$ At low concentrations of WPI fibrils, the emulsions showed regular creaming. Above a certain concentration ( $0.5 \mathrm{wt} \%$ for WPI fibrils prepared the same as this study), enhanced creaming was observed as induced by the attractive depletion interactions. ${ }^{1,22}$ The creaming rate decreased with increasing WPI fibrils concentration (from 0.5 to 2 wt\% WPI fibrils). Further increasing the WPI fibril concentration makes the emulsions become stabilized due to 'depletion stabilization'.1, ${ }^{11}$ This is clearly reflected in the emulsion containing 2.5 wt\% fibrils after 1 day of preparation, which showed higher stability than emulsion containing 2 wt\% fibrils. It is expected that with WPI fibrils higher than 2.5 wt\%, the emulsions will remain stable over time. ${ }^{1,21}$

In emulsions containing both WPI fibrils and BC microfibrils, we observe that at low BC microfibril concentration ( $0.005 \mathrm{wt} \%)$, the emulsions showed similar behaviour to those of emulsions containing only WPI fibrils. With increasing BC microfibril concentration up to $0.3 \mathrm{wt} \%$, for emulsions containing WPI fibril concentrations up to $0.1 \mathrm{wt} \%$, a similar stability profile to those of emulsions containing only BC microfibrils (Figure 3 ) is observed, i.e. the emulsion creaming is slowed down substantially at higher BC microfibril concentrations. Interestingly, for emulsions containing WPI fibrils concentration between 0.5 and 2 wt\%, where high extent of creaming was observed when no BC microfibrils are present, the creaming was gradually slowed down with increasing of BC microfibril concentration. At WPI fibril concentration of $2.5 \mathrm{wt} \%$ and BC microfibril concentration above 0.05 wt\%, the emulsions remained stable for 7 days. At a fixed BC microfibril concentration, the extent of creaming was further slowed down with increasing WPI fibril concentration from $0.5 \mathrm{wt} \%$ to $2.5 \mathrm{wt} \%$, similar to those of emulsions containing only WPI fibrils. However, for emulsions containing 0.5 and 1 wt\% WPI, the emulsions showed lower stability than emulsions containing WPI fibrils $\leq 0.1$ wt $\%$ and $\geq 2$ wt\%. At BC microfibril concentration of $0.35 \mathrm{wt} \%$, all emulsions that contain also WPI fibrils remained stable at 1 day after sample preparation. After 7 days, slight creaming was observed at the bottom of the sample, except for the sample containing both $0.35 \mathrm{wt} \% \mathrm{BC}$ microfibrils and 2 wt\% WPI fibrils, which remained stable. 


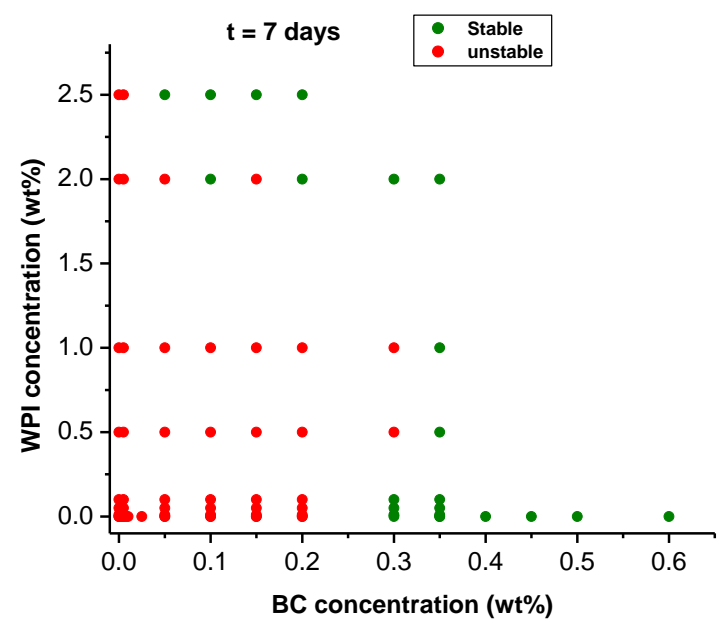

Figure 5 The stability diagram at $20^{\circ} \mathrm{C}$ of $10 \%(\mathrm{v} / \mathrm{v}) \mathrm{o} / \mathrm{w}$ emulsion containing both WPI fibrils and $\mathrm{BC}$ microfibrils at different concentration ratios at 7 days after sample preparation. Emulsions that are stable are indicated by the green spheres, while emulsions that are unstable against creaming are indicated by the red spheres.

The stability diagram after 7 days for emulsions containing different concentration ratios between WPI fibrils and BC microfibrils is given on the basis of the visual observations in Figures 3 and 4 . When both WPI fibril and BC microfibril concentrations are low, the emulsions are unstable after 7 days. When the WPI fibrils concentration is kept low, sufficient increase of the $B C$ microfibril concentration leads to a stable emulsion. When the BC microfibril concentration is kept low, adding WPI fibrils higher than 2 wt\% also leads to a stable emulsion. The stability of the emulsions can be tuned by varying the concentration ratios between WPI fibrils and BC microfibrils. 


\subsubsection{Rheological properties}
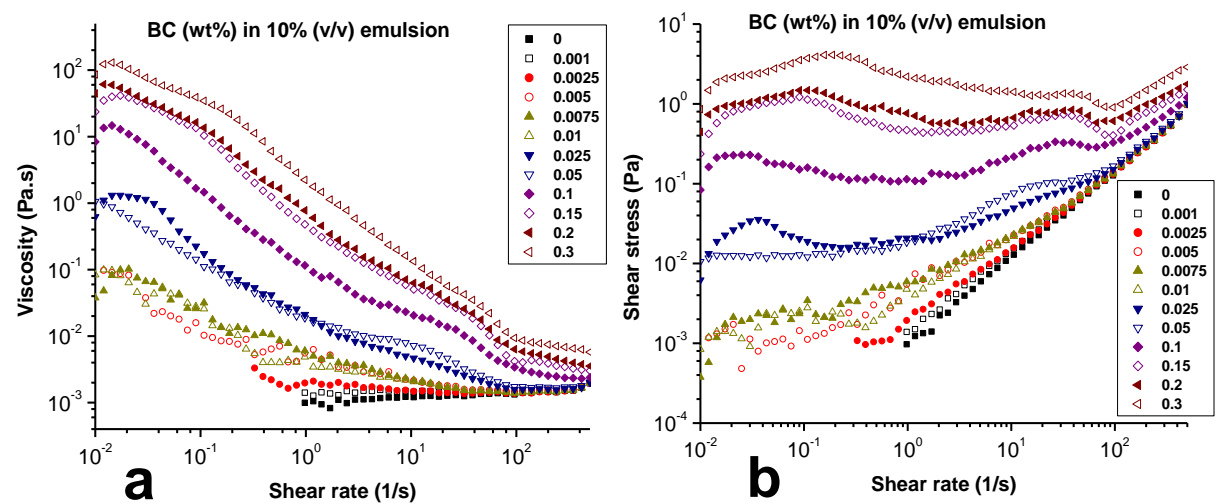

Figure 6 Viscosity (a) and shear stress (b) as a function of shear rate for $10 \%(\mathrm{v} / \mathrm{v})$ emulsions containing BC microfibrils at concentratons of $0,0.001,0.0025,0.005,0.0075,0.01,0.025,0.05$, $0.1,0.15,0.2$ and $0.3 \mathrm{wt} \%$.

In emulsions containing only BC microfibrils, we observed that at BC microfibril concentrations lower than $0.005 \mathrm{wt} \%$, the samples behave like the emulsion without BC microfibrils, i.e. Newtonian behaviour (Figure 6). At BC microfibril concentrations higher than $0.005 \mathrm{wt} \%$, the samples show shear thining behaviour. The viscosity increases with increasing $B C$ microfibril concentration. Plotting the shear stress over shear rate, we observed the same value of shear stress at different shear rates above BC concentrations of 0.025 wt\%, which may be attributed to shear banding behaviour of the BC microfibrils, as has been reported in a previous study on rheological properties of BC microfibrils prepared in the same way. ${ }^{34}$ In addition, the shear viscosity in our study is comparable to the viscosity of pure $B C$ microfibril dispersions in that previous study, which implies that the viscosity of the continuous phase of the emulsion containing $B C$ microfibrils is dominated by BC microfibrils.

In comparison to the storage modulus $\mathrm{G}^{\prime}$ and loss modulus $\mathrm{G}^{\prime \prime}$ of the pure BC microfibril dispersions (no emulsion), the $G^{\prime}$ and $G^{\prime \prime}$ values of the emulsions containing $B C$ microfibrils were slightly lower, as shown in Figure 7. 


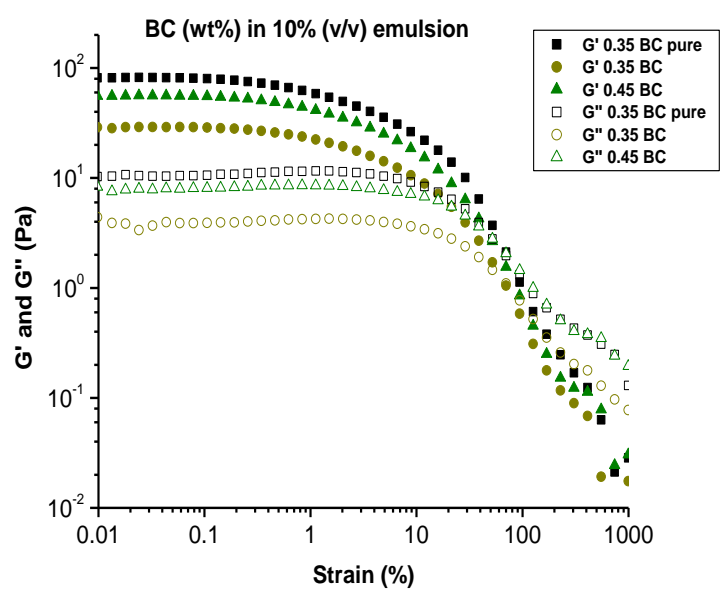

Figure 7 Storage modulus G'and loss modulus G' as a function of strain at a frequency of $\mathbf{1 ~ H z}$ for sample containing pure $0.35 \mathrm{wt} \% \mathrm{BC}$ microfibrils, and samples containing $10 \%(\mathrm{v} / \mathrm{v})$ emulsion and 0.35 or 0.45 wt $\%$ BC microfibrils.
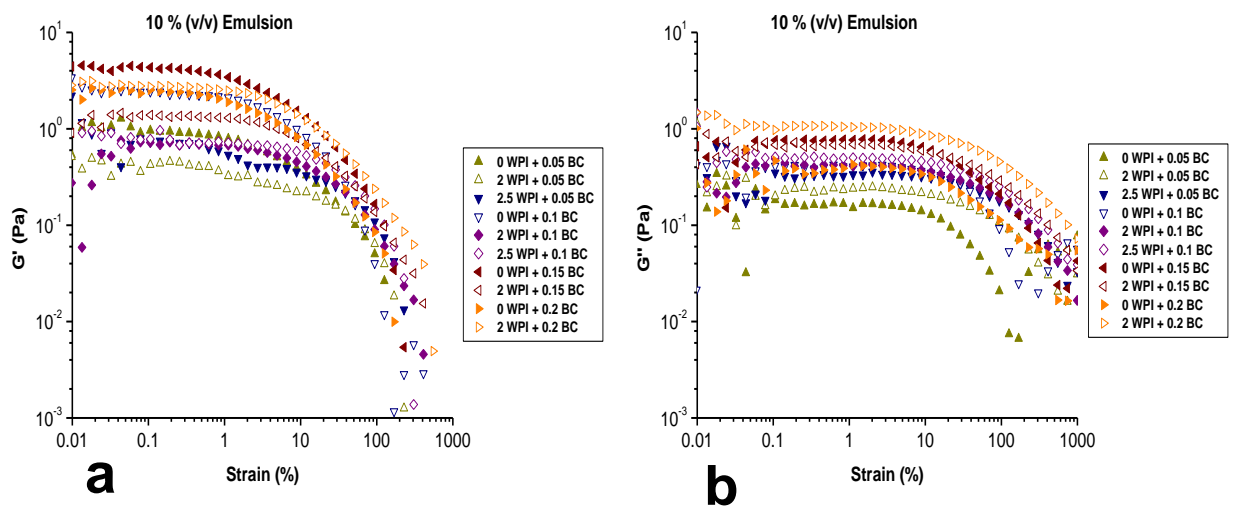

Figure 8 Storage modulus G' (a) and loss modulus G' (b) as a function of strain at a frequency of 1 $\mathrm{Hz}$ for samples containing $10 \%(\mathrm{v} / \mathrm{v}$ )emulsion and WPI fibrils (wt\%) and BC microfibrils (wt\%) at different cocnentration ratios. 
For the emulsions containing both WPI fibrils and BC microfibrils, the $\mathrm{G}^{\prime}$ and $\mathrm{G}^{\prime \prime}$ values are both low ( $<5 \mathrm{~Pa}$, Figure 8 ) and $\mathrm{G}^{\prime}$ is larger than $\mathrm{G}^{\prime \prime}$, due to the gel-like behaviour of BC microfibrils. ${ }^{34}$ The $G^{\prime}$ value increases with increase in BC microfibril and WPI fibril concentrations in the emulsions.The values, and their increase upon concentration increase of BC microfibrils and WPI fibrils, are similar to that for dispersions containing pure WPI fibrils or pure BC microfibrils (data from previous studies ${ }^{1,34}$ ). Therefore we conclude that the increase of $\mathrm{G}^{\prime}$ of the emulsions is mainly due the contributions from WPI fibril and BC microfibrils, and interactions with the emulsion droplets or in between emulsions droplets (forming an emulsion gel) is unlikely. We note that our studies on the rheology of WPI fibrils and BC microfibrils (in Chapter 3 ), did not reveal specific attractive interactions between WPI fibrils and BC microfibrils, at pH 2.

\subsubsection{Confocal laser scanning microscopy (CLSM)}

The top layer of the emulsions at 7 days after preparation were imaged using CSLM to investigate their microstructure. Figure 9 shows the CLSM images for emulsions containing only $\mathrm{BC}$ microfibrils and Figrues 10 -12 shows the CLSM images for emulsions containing both WPI fibrils and BC microfibrils. 


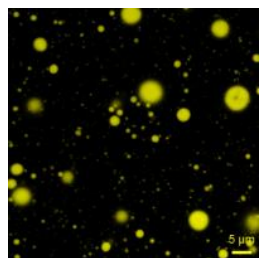

$0 \mathrm{wt} \%$

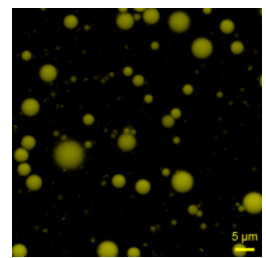

0.05 wt $\%$

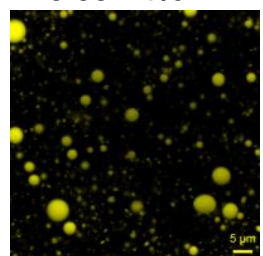

$0.3 w t \%$

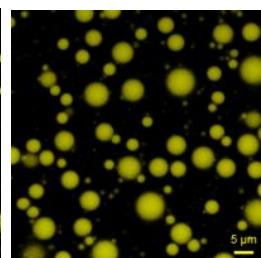

0.001 wt\%

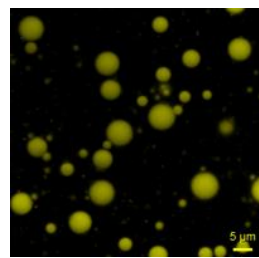

$0.1 \mathrm{wt} \%$

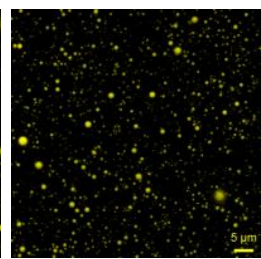

0.35 wt \%

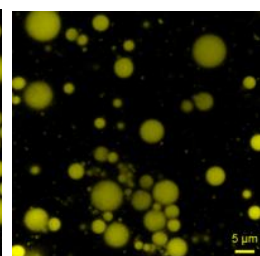

0.005 wt \%

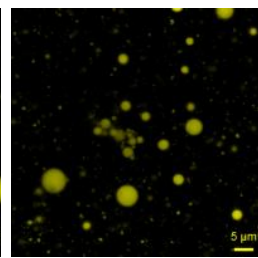

0.15 wt $\%$

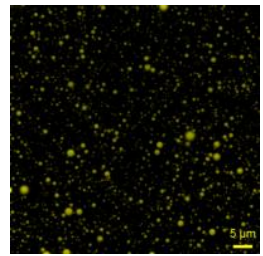

0.4 wt\%

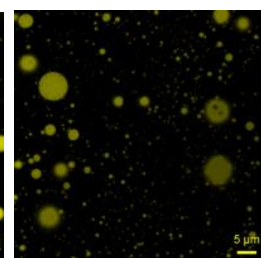

0.025 wt $\%$

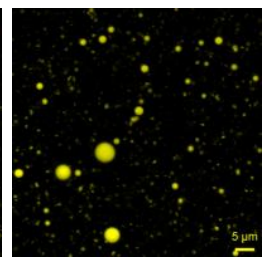

$0.2 \mathrm{wt} \%$

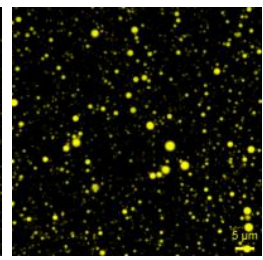

0.45 wt \%

Figure 9 CLSM images of the top layer of $10 \%(\mathrm{v} / \mathrm{v})$ emulsions containing $\mathrm{BC}$ microfibrils at concentrations (indicated below the image) of $0,0.001,0.005,0.025,0.05,0.1,0.15,0.2,0.3,0.35$, 0.4 and 0.45 wt\% at $20^{\circ} \mathrm{C}$ at 7 days after preparation. The oil droplets are shown in yellow. Scale bar corresponds to $5 \mu \mathrm{m}$.

In the emulsions containing only BC microfibrils, no oil droplet aggregation could be observed (Figure 9) and the oil droplets were homogeneously distributed in the top layer. At low BC microfibril concentrations ( $\leq 0.1 \mathrm{wt} \%$ ), the microstructure of the emulsions is similar to that of the emulsion without BC microfibrils. Above $0.15 \mathrm{wt} \% \mathrm{BC}$ microfibrils, more smaller droplets were observed in the top layer of the emulsions. At BC mirofibril concentrations $\geq 0.35 \mathrm{wt} \%$, almost all oil droplets are small, in contrast to emulsions with lower BC microfibril concentrations. Apparently the larger droplets are trapped within the $\mathrm{BC}$ microfibril network and can not cream towards the top layer of the sample, while the smaller droplets still can cream. Macroscopic images showed that these emulsions are stable for 7 days (Figure 3). 


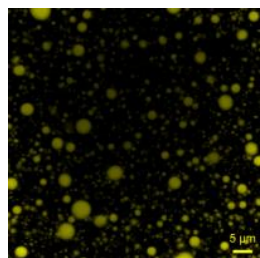

$0.005 \mathrm{wt} \%$

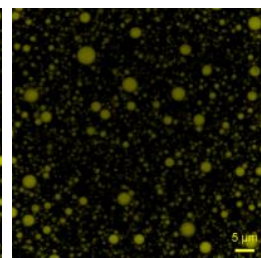

$0.1 \mathrm{wt} \%$

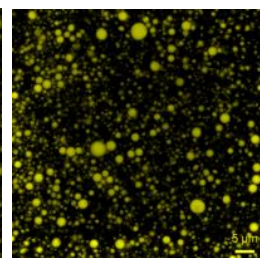

$0.3 \mathrm{wt} \%$

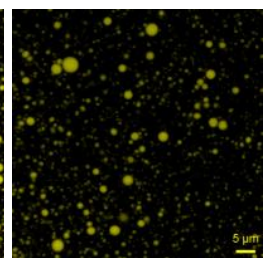

$0.35 \mathrm{wt} \%$

Figure $10 \mathrm{CLSM}$ images of the top layer of $10 \%(\mathrm{v} / \mathrm{v})$ emulsions at $20^{\circ} \mathrm{C}$ at 7 days after preparation. The emulsions contain 1 wt\% WPI fibrils and BC microfibrils at concentrations of $0.005,0.1,0.3$ and $0.35 \mathrm{wt} \%$ as indicated below the images. The oil droplets are shown in yellow. The scale bar corresponds to $5 \mu \mathrm{m}$.

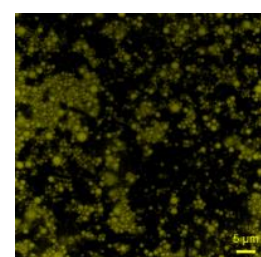

$0 \mathrm{wt} \%$

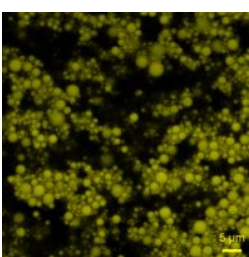

0.005 wt \%

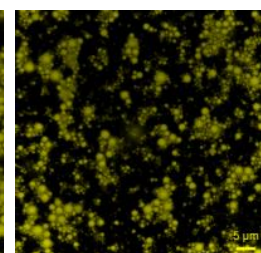

$0.1 \mathrm{wt} \%$

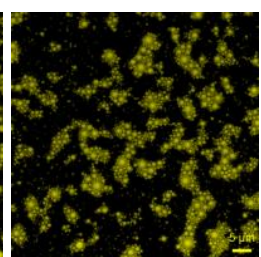

$0.3 \mathrm{wt} \%$

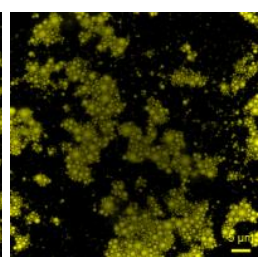

$0.35 \mathrm{wt} \%$

Figure $11 \mathrm{CLSM}$ images of the top layer of $10 \%(\mathrm{v} / \mathrm{v})$ emulsions at $20^{\circ} \mathrm{C}$ at 7 days after preparation. The emulsions contain 2 wt\% WPI fibrils and BC microfibrils at concentrations of $0,0.005,0.1,0.3$ and $0.35 \mathrm{wt} \%$ as indicated below the images. The oil droplets are shown in yellow. Scale bar corresponds to $5 \mu \mathrm{m}$.

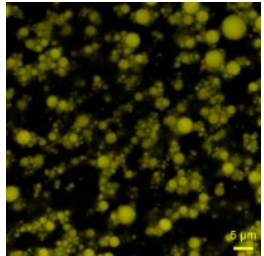

$0 \mathrm{wt} \%$

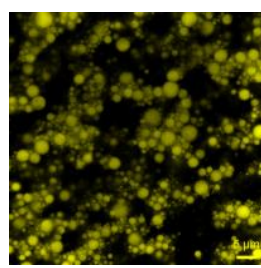

$0.005 w t \%$

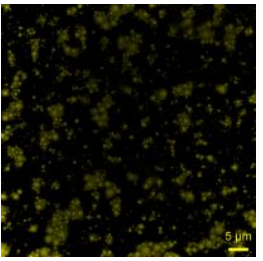

$0.1 \mathrm{wt} \%$

Figure 12 CLSM images of the top layer of $10 \%(\mathrm{v} / \mathrm{v})$ emulsions at $20^{\circ} \mathrm{C}$ at 7 days after preparation. The emulsions contain $2.5 \mathrm{wt} \%$ WPI fibrils and BC microfibrils at concentrations of $0,0.005$, and 0.1 wt\% as indicated below the image. The oil droplets are shown in yellow. Scale bar corresponds to $5 \mu \mathrm{m}$.

In Figrue 10, CLSM images of the top layer of emulsions with 0.1 wt\% WPI fibrils and BC microfibrils at different concentrations are shown. All emulsions showed a similar 
microstructure with oil droplets homogeneously distributed in the top layer, with comparable size to that of the bare emulsion (Figure 9). However, it was noted that at BC microfibril concentraiton higher than $0.3 \mathrm{wt} \%$, more small oil droples are observed.

At fixed WPI fibril concentration of 2 wt\%, as shown in Figure 11, oil droplet aggregation due to depletion flocculation ${ }^{1,22}$ was observed in emulsions with 0 wt\% BC microfibril concentration. Upon addition of BC microfibrils, the oil droplet aggregation still occurred, regardless of the $B C$ microfibril concentration used. This suggests that the presence of $B C$ microfibrils did not prevent the oil aggregation due to depletion floccuation induced by WPI fibrils. However, emulsions containing 2 wt\% WPI fibrils and BC microfibril concentration of $0.1,0.3$ and 0.35 wt\% remaind macroscopically stable, as shown in Figure 4.

At 2.5 wt\% WPI fibrils, all emulsions showed oil droplet aggregation (Figure 12), but the aggregate sizes are generally smaller than the oil droplet aggregates in emulsions containing 2 wt\% WPI fibrils, at the same BC mcirofibril concentration. The phenomenon of the oil droplet aggreation in the emulsions as a function of WPI fibril concentration is similar to that observed in emulsions containing only WPI fibrils. ${ }^{1}$ This was attributed to depletion interaction induced by the WPI fibrils. With increase in WPI fibril concentration, the enhanced creaming due to depletion flocculation decreased. Above a certain concentration of WPI fibrils, depletion stabilization takes places, with the emulsion droplets present in the form of both single droplets and droplet clusters. ${ }^{1,21}$ The results imply that oil droplet aggregation induced by WPI fibrils was not prevented by the incorporation of $B C$ microfibrils. Macroscopic images (Figure 4) show a stable sample above a certain $B C$ microfibril concentration, suggesting that the $B C$ microfibrils are able to stabilize the emulsions which otherwise would be unstable, within a certain range of WPI fibril concentrations.

We have characterized the stability of emulsions with the addition of $\mathrm{BC}$ microfibrils and with the addition of WPI fibrils and BC microfibrils (i.e. bifibrillar WPI-BC networks) as a function of the respective fibril concentrations using rheology and microscopy. The emulsion stability in the presence of only WPI fibrils had been studied previously (i.e. Chapter 6). ${ }^{1,22}$ Results showed that at low concentrations of WPI fibrils, depletion flocculation was induced, causing droplets aggregation, resulting in enhanced creaming. ${ }^{1}$

${ }^{22}$ We found that above a certain WPI fibril concentration, the WPI fibrils stabilize the 
emulsion. ${ }^{1}$ In these stabilized emulsions both aggregates and single droplets are present. The aggregates seeem to have a maximum size. The emulsions with WPI fibrils remained a low-viscosity fluid, i.e. no emulsion gel or fibril gel was observed. Moreover, the emulsions were found to remain stable at $45{ }^{\circ} \mathrm{C}$, implying a temperature independent stabilisation mechanism. Although the mechanism of the stabilization remains unclear, the results pointed into the direction of the presence of a kinetic barrier. Similar results were also found in colloidal dispersions containing oppositely charged polystyrene latex particles (i.e. Chapter 7). ${ }^{21}$

When only $B C$ microfibrils are added to emulsions, at low BC microfibril concentrations, the emulsions showed similar creaming behaviour to the emulsion without BC microfibrils. Upon increasing the $\mathrm{BC}$ microfibril concentration, the creaming behaviour is gradually slowed down, leaving a thin serum layer at the bottom of the sample. Above a certain BC microfibril concentration ( 0.4 wt\%), emulsions remained stable for 7 days after preparation. The microstructure from CLSM reveals that more smaller droplets are present in the top layer of the emulsions upon increasing BC microfibril concentrations. This in contrast to emulsions at low BC microfibril concentrations, where mainly large droplets are observed in the top layer. It was estimated that the minimum concentration of self-supporting gel formation of $\mathrm{BC}$ microfibrils is $~ 0.1 \%{ }^{34}$ This corresponds to the transition concentration of $\mathrm{BC}$ in emulsions where more small droplets are observed in the top layer of the emulsions (Figure 9). In emulsions without BC microfibrils, larger droplets creamed faster than smaller droplets. The results suggest that the larger droplets are probably trapped in the $B C$ microfibril gel network, while the small droplets can move through the BC microfibril network and reach the top layer. Interestingly, the rheological properties (i.e. viscosity and visco-elastic properties) of the emulsions were determined to be comparable to that of pure BC microfibrils at the same concentration. No droplet gel network structure or aggregation was observed. These results therefore exclude the possibility of stabilization by emulsion gel formation. Based on the above, we can draw some conclusions on the possible mechanisms of stabilisation due to BC microfibrils. Increasing the $\mathrm{BC}$ microfibril concentration, the viscosity also increase substaintially, ${ }^{34}$ as is also confirmed by the viscosity of emulsions containing BC microfibrils. The stabilization of emulsions by $B C$ microfibrils however cannot be attributed to the viscosity increase of the continuous phase of the emulsion, since this would contradict with the presence of smaller droplets at the top layer of the emulsions at higher BC microfibril concentration. 
The presence of the smaller droplets at the top layer can be explained by the mesh size of the network being such that it only allows the small droples to pass through (schematic picture in Figure 13). The presence of a few large droplets at the top layer of the emulsions with high concentration of $\mathrm{BC}$ microfibrils could be due to the heterogeneity of the $\mathrm{BC}$ network. ${ }^{34}$ The presence of large non-flocculated droplets throughout the $\mathrm{BC}$ network can be attributed to a yield stress, which is known to exist in these systems and which increases with increasing $B C$ microfibril concentration. ${ }^{29,34}$ Indeed, observing the motion of the droplets using CLSM over a time period of 2 minutes did not show displacement of the droplets over their radius implying the dropets are basically stuck. It is estimated that in order to prevent creaming of a droplet with size below $\sim 1 \mu \mathrm{m}$, a yield stress below $10^{-3} \mathrm{~Pa}$ would already be sufficient. ${ }^{41}$ For our type of BC microfibril systemsindeed a much higher yield stress of the order of $1 \mathrm{~Pa}$ was determined previously. 34

In the case of emulsions containing both WPI fibrils and BC microfibrils, it was shown that the emulsion stability can be tuned depending on the concentration ratios between WPI fibrils and BC microfibrils. Importantly, in emulsions with both types of fibrillar structure present, we oberved that the systems share the similar characteristics that also belong to the emulsions stabilized by WPI fibrils and BC microfibrils alone. More specifically, at a fixed $B C$ microfibril concentration, with increasing WPI fibril concentration in the mixture, we observed a trend of the emulsion stability as a function of WPI fibrils similar to that for the emulsion containing only WPI fibrils. At low WPI fibril concentration, oil droplet aggregation due to depletion floccuation induced by fibrils was observed. Above a certain fibril concentration, the oil droplet aggregates become smaller and emulsions are stabilized with both small aggregates and single droplets present together, due to the depletion stabilization. ${ }^{1,21}$ This trend is independent of BC microfibril concentration. Moreover, at higher $\mathrm{BC}$ microfibril concentration, the emulsions remained stable with the occurence of droplet aggregates induced by depletion flocculation, indicating that the presence of $B C$ microfibrils did not influence the behaviour induced by WPI fibrils in emulsion, and that the $\mathrm{BC}$ microfibrils network is able to stabilize the oil droplet aggregates. The creaming is gradually slowed down while increasing the BC microfibril concentration, at a fixed WPI fibril concentration.

When the BC microfibril concentration is high enough, the emulsions remained stable. This effect is also independent of WPI fibrils, regardless of the oil droplet aggreagtion 
induced by WPI fibrils. This indicates that BC microfibrils did not influence the oil droplet aggregation as induced by protein fibrils. It is more that the $\mathrm{BC}$ microfibrils are efficient to form a network to prevent the oil droplet aggregates from creaming.

In summary, the BC microfibrils act as a stabiliser via their formation of a network with a yield stress and does not act as a depletiong agent. The WPI fibrils do not form a network exerting a yield stress but are very effective in causing depletion, and cause depletion stabilisation at higher concentrations. An emulsion stabilised by WPI fibrils remains liquid like, while an emulsion stabilised by $\mathrm{BC}$ microfibrils becomes a yield stress fluid.

Although the mechanism of the stabilization and /or destabilization is different from each other, the competition between stabilization and/or destabilization induced by WPI fibrils and $\mathrm{BC}$ microfibrils alone at certain concentrations determines the final stability of the emulsions. Due to this competition, we could achieve emulsion stabilization and/or destabilization with different microstructures and rheological properties by varying the concentration ratios between the two types of fibrillar structures.

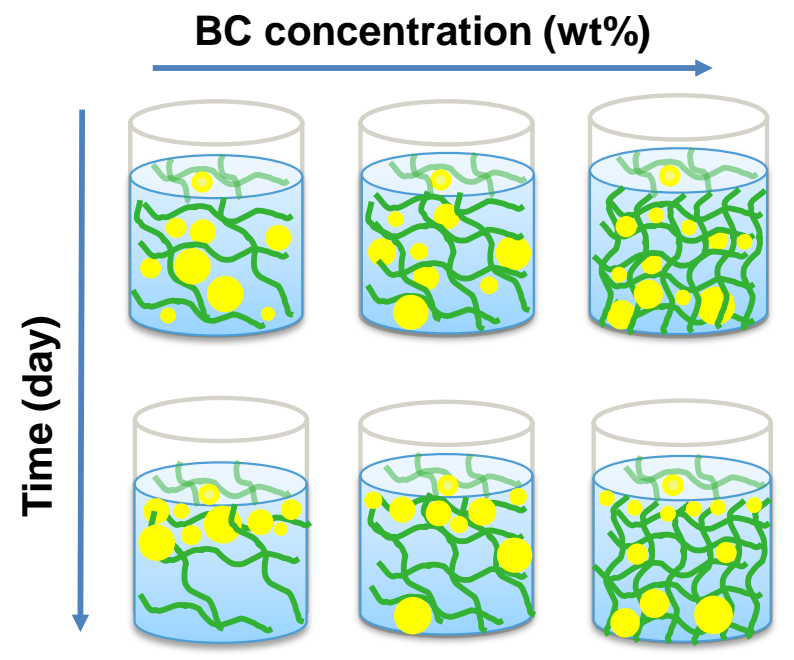

Figure 13 Schematic representation of the stabilising mechanism of BC microfibrils in o/w emulsions.

Temperature and salt concentration are critical parameters for emulsion stability. To investigate how the emulsion stability containing only $\mathrm{BC}$ microfibrils and the combination 
of WPI-BC bifibrillar networks is influenced by those parameters, the emulsion stability was investigated at different temperatures and salt concentrations.

\subsubsection{Effect of temperature on emulsion stability}

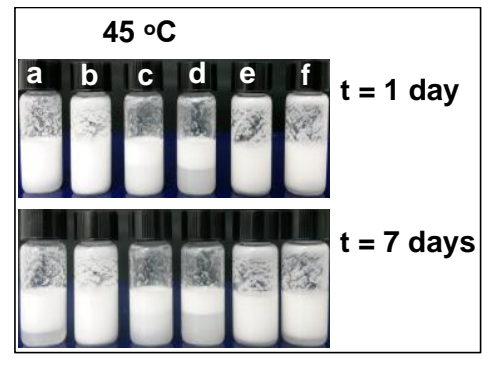

a

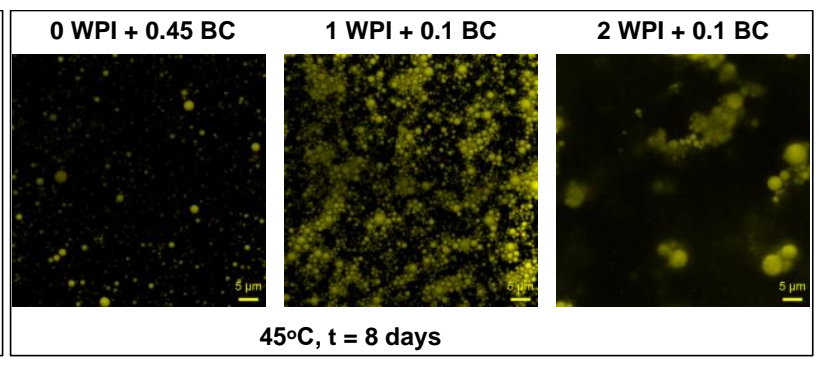

b

Figure 14 (a) shows the images of a $10 \%$ (v/v) emulsion containing BC microfibrils and/or WPI fibrils at $45^{\circ} \mathrm{C}$ at 1 and 7 days after preparation. Emulsions a and b contain only BC mirofibrils with concentration of 0.2 (a) and 0.45 wt\% (b). Emulsions c-f contain both WPI fibrils and BC microfibrils with concentration ratios: 1 wt $\%$ WPI $+0.1 w t \%$ BC (c), 2 wt\% WPI + 0.1 wt\% BC (d), 1 $w t \% ~ W P I+0.35 w t \% ~ B C(e), 2 w t \%$ WPI + $0.35 w t \% ~ B C(f)$. The sample volume is half of the bottle volume and the emulsion residue on the upper part of the bottle is caused by mixing. (b) shows the corresponding CLSM images of a, $c$ and $d$ samples after 8 days at $45^{\circ} \mathrm{C}$. The concentrations (wt\%) are indicated on the top of the images.

Emulsions that are stored at $45^{\circ} \mathrm{C}$ (Figure 14) in the oven showed similar stability to the emulsions at $20^{\circ} \mathrm{C}$, as shown in Figures 3 and 4. Microscopic images for emulsions containing $0.45 \mathrm{wt} \% \mathrm{BC}$ microfibrils showed a homogeneous droplets distribution on the top of the samples with mainly small droplets present, similar to the emulsion at $20^{\circ} \mathrm{C}$ (Figure 9). For emulsions containing both WPI fibrils and BC microfibrils, we observed droplet aggregation for both emulsions containing 1 and 2 wt\% WPI fibrils, with a BC microfibril concentration of $0.1 \mathrm{wt} \%$. This is comparable to those of the emulsions stored at $20{ }^{\circ} \mathrm{C}$ (Figures 10-11), in which droplet aggregation was attributed to depletion flocculation as induced by the protein fibrils. These results imply that temperature did not have a significant influence on the emulsion stability. Emulsions stabilized by only BC microfibrils or a mixture of WPI fibrils and BC microfibrils are both heat stable up to temperatures of at least $45^{\circ} \mathrm{C}$. The heat stability of emulsions in the presence of only WPI 
fibrils has been found earlier. ${ }^{1,21}$ In addition, BC microfibrils has been found stable against heating ${ }^{26}$ and has been shown to be a heat-stable suspending agent in chocolate drink. ${ }^{25}$

\subsubsection{Effect of $\mathrm{NaCl}$ on emulsion stability}

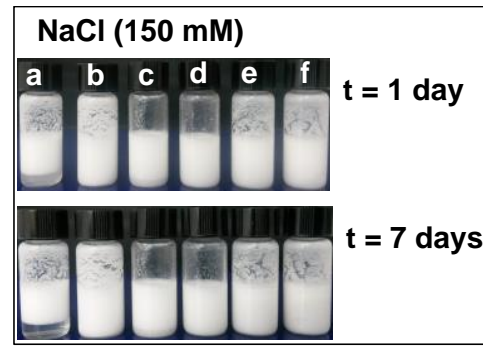

a

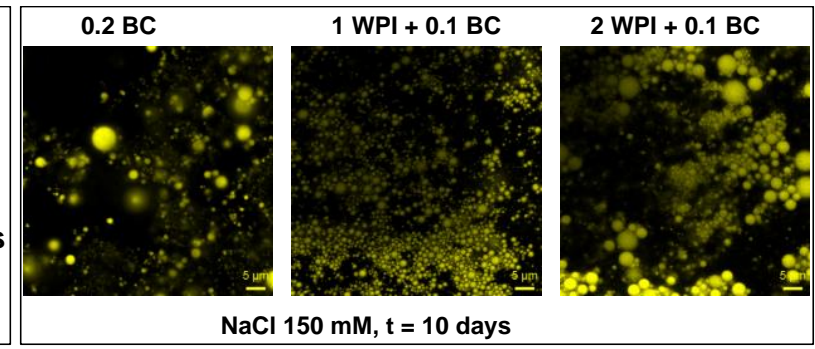

b

Figure 15 (a) shows images of a 10\% (v/v) emulsion containing BC microfibrils and/or WPI fibrils with $150 \mathrm{mM} \mathrm{NaCl}$ at 1 and 7 days after preparation. Emulsions $a$ and $b$ contain only BC mirofibrils with concentration of 0.2 (a) and 0.45 wt\% (b). Emulsions c-f contain both WPI fibrils and BC microfibrils with concentration ratios: 1 wt $\%$ WPI +0.1 wt $\%$ BC (c), 2 wt $\%$ WPI + 0.1 wt\% BC (d), 1 $w t \% ~ W P I+0.35 w t \% ~ B C(e), 2 w t \%$ WPI + $0.35 w t \% ~ B C(f)$. The sample volume is half of the bottle volume and the emulsion residue on the upper part of th bottle is caused by mixing. (b) shows the corresponding CLSM images of emulsions a, $\mathrm{c}$ and $\mathrm{d}$ after 10 days. The concentrations (wt\%) are indicated on the top of the images.

The emulsions containing only $\mathrm{BC}$ microfibrils with addition of $150 \mathrm{mM} \mathrm{NaCl}$ showed a similar stability to emulsions without $\mathrm{NaCl}$ ( Figure 3). The microstructure of the oil droplets on the top of the emulsion containing $0.2 \mathrm{wt} \% \mathrm{BC}$ microfibrils is also comparable to the emulsion without $\mathrm{NaCl}$ (Figure 9). No oil droplet aggregation could be clearly observed. The emulsions containing both WPI fibrils and BC microfibrils were still stable after 7 days. It was noted, that these emulsions showed even better stability (no creaming was obserd in all emulsions) in comparison to emulsions without $\mathrm{NaCl}$ (Figure 4). Oil droplet aggregation was observed in both emulsions containing 1 and 2 wt\% WPI fibrils with a fixed $\mathrm{BC}$ concentration of $0.1 \mathrm{wt} \%$, which is similar to that for emulsions without $\mathrm{NaCl}$ (Figure 10-11).

Previous research has found that the structure of BC microfibrils is not affected by salt addition. ${ }^{26}$ This might explain the similar stability of emulsion stabilized by BC microfibrils 
with 0 and $150 \mathrm{mM} \mathrm{NaCl}$. WPI fibrils and the WPI-stabilized oil droplets are charged at $\mathrm{pH}$ 2 , thus the charge screening effect from $\mathrm{NaCl}$ can cause aggregation of WPI fibrils and also aggregation between the oil droplets. This will lead to higher viscosity of the emulsion and possibly even gelation of the emulsion. It has been reported that the increase of $\mathrm{NaCl}$ concentration in $\beta$-lg fibrils induces phase transitions from nematic to gel phases. ${ }^{42}$ Upon even higher $\mathrm{NaCl}$ cocnentration, one may observe a transition to a translucent phase and macroscopic phase separation. Therefore, the delayed creaming of emulsions at $150 \mathrm{mM}$ $\mathrm{NaCl}$ containing both WPI fibrils and BC microfibrils may be explained by the increased viscosity of the WPI fibril network.

\subsection{Conclusion}

$\mathrm{BC}$ microfibrils stabilise emulsions above a certain concentration. The BC microfibrils form a yield stress fluid that is able to prevent creaming of larger droplets and aggregates, while smaller droplets may be diffusing through the mazes of the BC microfibrillar network. WPI fibrils are known to induce depletion flocculation, and, upon increasing fibril concentration also depletion stabilization. Therefore, BC microfibrils and WPI fibrils play different roles in emulsions regarding stabilizing and destabilizing them. Emulsion stability and rheological properties can be tuned by varying the relative concentrations of WPI fibrils and BC microfibrils. As a result, the competition between stabilization and/or destabilization induced by both WPI fibrils and BC microfibrils leads to different emulsion stability with different microstructures and rheological properties.

\subsection{Acknowledgement}

This work is supported by NanoNextNL, a micro and nanotechnology consortium of the Government of the Netherlands and 130 partners. We thank Sandra J. Veen for providing the dispersions of bacterial cellulose and TEM analysis. We thank Harry Baptist for TEM analysis and Mirada de Beus for CLSM analysis. 


\subsection{References}

1. J. Peng, J. R. Simon, P. Venema and E. van der Linden, Langmuir, 2016, 32, 2164-2174.

2. E. Dickinson, Food Hydrocolloids, 2003, 17, 25-39.

3. E. Dickinson and V. B. Galazka, Gums and stabilisers for the food industry, 1992, 6, 351-362.

4. E. Dickinson, Food Hydrocolloids, 2009, 23, 1473-1482.

5. A. Parker, P. A. Gunning, K. Ng and M. M. Robins, Food Hydrocolloids, 1995, 9, 333-342.

6. R. I. Feigin and D. H. Napper, Journal of Colloid and Interface Science, 1980, 75, 525-541.

7. S. Asakura and F. Oosawa, The Journal of Chemical Physics, 1954, 22, 1255-1256.

8. S. Asakura and F. Oosawa, Journal of Polymer Science, 1958, 33, 183-192.

9. C. M. Dobson, Nature, 2003, 426, 884-890.

10. M. R. H. Krebs, G. L. Devlin and A. M. Donald, Biophysical Journal, 2007, 92, 1336-1342.

11. E. van der Linden and P. Venema, Current Opinion in Colloid \& Interface Science, 2007, 12, 158165.

12. C. Akkermans, A. J. van der Goot, P. Venema, E. van der Linden and R. M. Boom, Food Hydrocolloids, 2008, 22, 1315-1325.

13. C. Akkermans, A. J. Van der Goot, P. Venema, H. Gruppen, J. M. Vereijken, E. Van der Linden and R. M. Boom, Journal of Agricultural and Food Chemistry, 2007, 55, 9877-9882.

14. C. D. Munialo, A. H. Martin, E. van der Linden and H. H. J. de Jongh, Journal of Agricultural and Food Chemistry, 2014, 62, 2418-2427.

15. C. Veerman, G. de Schiffart, L. M. C. Sagis and E. van der Linden, International Journal of Biological Macromolecules, 2003, 33, 121-127.

16. L. N. Arnaudov and R. de Vries, Biophysical Journal, 2005, 88, 515-526.

17. G. M. Kavanagh, A. H. Clark and S. B. Ross-Murphy, International Journal of Biological Macromolecules, 2000, 28, 41-50.

18. C. Akkermans, P. Venema, A. J. van der Goot, H. Gruppen, E. J. Bakx, R. M. Boom and E. van der Linden, Biomacromolecules, 2008, 9, 1474-1479.

19. E. H. C. Bromley, M. R. H. Krebs and A. M. Donald, Faraday Discussions, 2005, 128, 13-27.

20. A. Kroes-Nijboer, P. Venema and E. van der Linden, Food \& function, 2012, 3, 221-227.

21. J. Peng, A. Kroes-Nijboer, P. Venema and E. van der Linden, Soft Matter, 2016, 12, 3514-3526.

22. T. B. J. Blijdenstein, C. Veerman and E. van der Linden, Langmuir, 2004, 20, 4881-4884.

23. Z. Shi, Y. Zhang, G. O. Phillips and G. Yang, Food Hydrocolloids, 2014, 35, 539-545.

24. A. Okiyama, M. Motoki and S. Yamanaka, Food Hydrocolloids, 1992, 6, 479-487.

25. A. Okiyama, M. Motoki and S. Yamanaka, Food Hydrocolloids, 1993, 6, 503-511.

26. H. Ougiya, K. Watanabe, Y. Morinaga and F. Yoshinaga, Bioscience, Biotechnology, and Biochemistry, 1997, 61, 1541-1545. 
27. H. Zhu, S. Jia, H. Yang, W. Tang, Y. Jia and Z. Tan, Food Science and Biotechnology, 2010, 19, 1479-1484.

28. P. Paximada, E. Tsouko, N. Kopsahelis, A. A. Koutinas and I. Mandala, Food Hydrocolloids, 2016, 53, 225-232.

29. P. Paximada, A. A. Koutinas, E. Scholten and I. G. Mandala, Food Hydrocolloids, 2016, 54, 245254.

30. S. Cho and N. Almeida, Dietary fiber and health, CRC Press, 2012.

31. K. Lin and H. Lin, Journal of Food Science, 2004, 69, SNQ107-SNQ111.

32. S. B. LIN, L. C. CHEN and H. H. CHEN, Journal of Food Process Engineering, 2011, 34, 1363-1379.

33. M. Phisalaphong and N. Chiaoprakobkij, Bacterial Cellulose: A Sophisticated Multifunctional Material, 2012, 9, 143-156.

34. A. Kuijk, R. Koppert, P. Versluis, G. van Dalen, C. Remijn, J. Hazekamp, J. Nijsse and K. P. Velikov, Langmuir, 2013, 29, 14356-14360.

35. A. Okiyama, M. Motoki and S. Yamanaka, Food Hydrocolloids, 1993, 6, 493-501.

36. S. J. Veen, A. Kuijk, P. Versluis, H. Husken and K. P. Velikov, Langmuir, 2014, 30, 13362-13368.

37. L. N. Arnaudov, R. de Vries, H. Ippel and C. P. M. van Mierlo, Biomacromolecules, 2003, 4, 16141622.

38. J.-M. Jung and R. Mezzenga, Langmuir, 2009, 26, 504-514.

39. R. Mezzenga, J.-M. Jung and J. Adamcik, Langmuir, 2010, 26, 10401-10405.

40. U. Geyer, T. Heinze, A. Stein, D. Klemm, S. Marsch, D. Schumann and H. P. Schmauder, International Journal of Biological Macromolecules, 1994, 16, 343-347.

41. E. Dickinson, Food structure: its creation and evaluation/[edited by] JMV Blanshard, JR Mitchell, 1988.

42. S. Bolisetty, L. Harnau, J.-m. Jung and R. Mezzenga, Biomacromolecules, 2012, 13, 3241-3252. 


\section{Chapter 9}

\section{General discussion}


Chapter 9 


\subsection{Introduction}

This thesis aims to better understand the behaviour of mixtures containing fibrillar and spherical structures. As fibrillar structures, bacterial cellulose (BC) microfibrils and whey (WPI) protein fibrils are used. As spherical structures, polystyrene latex particles and emulsion droplets are used as spherical structures. Fibrillar structures are a common type of structure in foods; xanthan and carragenaan are examples of such structures. They offer different physico-chemical properties compared to other structures like spherical particles. ${ }^{1}$ For example, their high aspect ratio (length versus diameter) leads to high excluded volume. They can be rigid, semi-flexible or flexible. ${ }^{2}$ As a result, rich phase behaviour can be induced by fibrillar structures in mixed systems containing other structures like spheres.

The behaviour of the various mixtures can be illustrated (cf. Figure 1) using a triangle with three corner points that present the main structural elements used in this thesis: bacterial cellulose microfibrils, whey protein isolate (including protein fibrils) and spheres (polystyrene latex particles or emulsions droplets). Each point within the triangle denotes a particular ratio of sphere/WPI/BC microfibrils. The various pictures within the figure schematically represent the properties and structures that are within the given region in the triangle. 


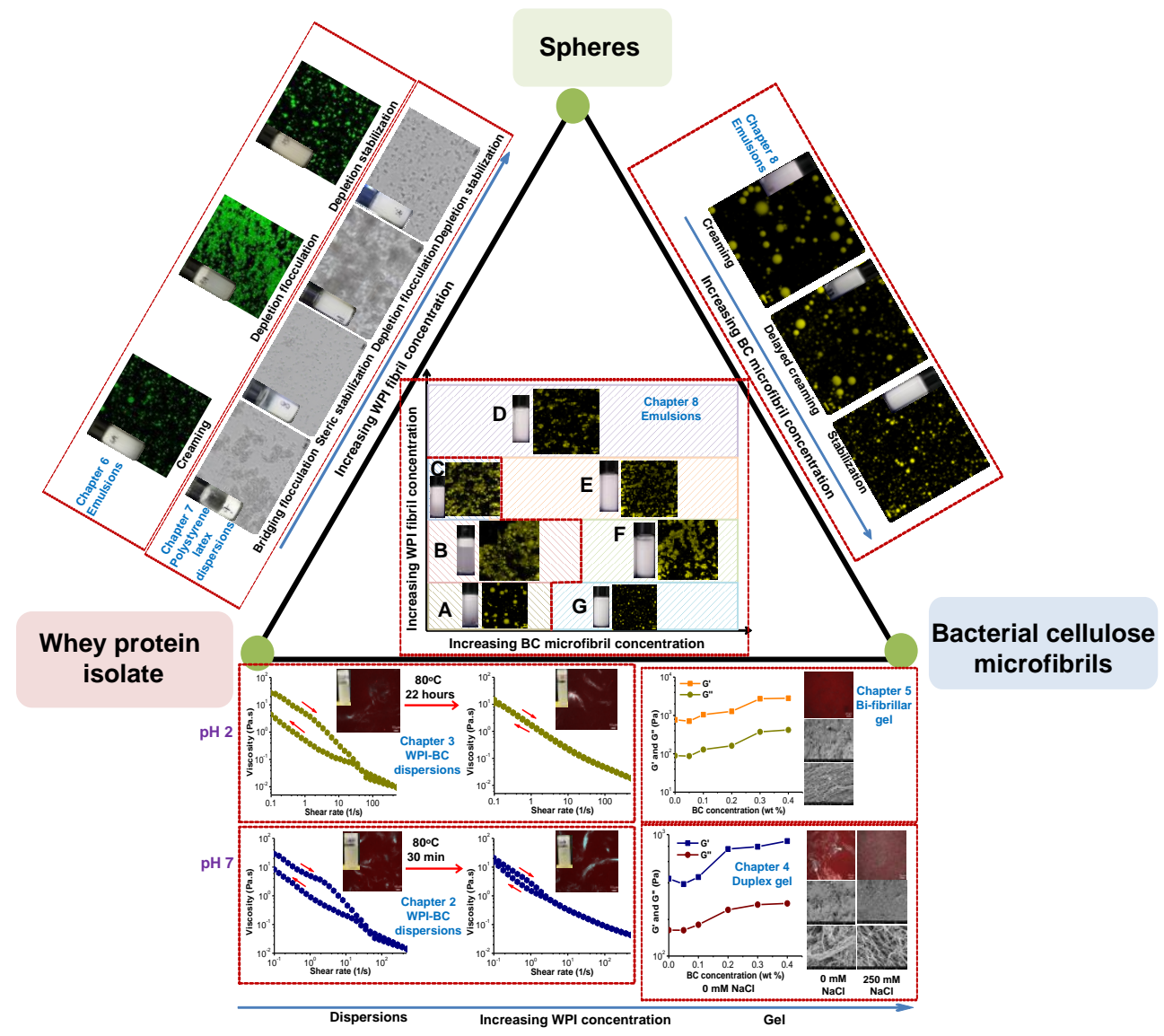

Figure 1 Schematic representation of the compositions studied in the thesis and their main features summarised in images that are situated in the according regions of the triangle.

Before discussing the main findings for each of the systems studied in this thesis and their interdependencies, we first discuss the properties of the WPI (including fibrils) and BC microfibrillar systems separately.

\subsubsection{Whey proteins and fibrils}

Whey proteins play an important role in food industry because of their nutritional value and versatile functional properties. ${ }^{3,4}$ Their ability to form different structures under certain conditions have broadened their functionality ranging from use as thickening, gelling, stabilizing to foaming and emulsifying agents. ${ }^{3}$ Whey protein isolate (WPI) contains 
a mixture of proteins with $\beta$-lactoglobulin $(\beta-\mathrm{lg})$ as the main protein. ${ }^{5}$ Depending on the conditions, structures from WPI can form that range from linear, branched to spherical aggregates. ${ }^{6,7}$ When WPI is heated at a pH close to the iso-electric point pl, where the net protein charge is low, spherical aggregates, also referred to as particulates, are formed. These particulates form a so-called particulate gel. ${ }^{8,9}$ When WPI is heated at a $\mathrm{pH}$ far from the $\mathrm{pl}$ and low ionic strength, i.e. when the electrostatic repulsion is strong, fine-stranded structures are formed. ${ }^{6,10,11}$ Upon increasing the salt concentration, the structure of the protein aggregates changes from fine-stranded to particulate. ${ }^{12,13}$

When WPI is heated at $\mathrm{pH} 2$ at $80^{\circ} \mathrm{C}$ at low ionic strength for several hours, semi-flexible rod-like protein fibrils can be formed. ${ }^{14,15}$ The formation process is composed of two steps: 1) the protein hydrolyzes to peptides; 2 ) a part of the peptides assembles into fibrils. ${ }^{16}$ The formed fibrils are polydisperse in length, which is in the order of micrometres. ${ }^{17,18}$ Their thickness is about $4 \mathrm{~nm}^{19}$ and the persistence length is around one micrometer. ${ }^{18,20}$ Their high aspect ratio (length versus diameter) makes them interesting candidates as structuring agents in foods. Previous research has reported that the fibrils can be used as a thickening, ${ }^{21}$ gelling, ${ }^{22-25}$ and foaming agent. ${ }^{26}$ Their use in production of microcapsules has also been explored. ${ }^{27,28}$ In addition, the fibrils are effective depletion agents. ${ }^{29-31}$ With increase of the fibril concentration, four different regimes can be identified: dilute, semidilute, isotropic and a nematic regime. ${ }^{18,32,33}$

The ability of proteins to form fibrils, via a hydrolysis step forming peptides that actually build the fibrils, is a generic characteristic of proteins. ${ }^{34,35}$ Many other proteins have been found that form fibrils according to this hydrolysis route. ${ }^{36-40}$ The conversion of proteins into fibrils and the formed fibril structures can be affected by the conditions used, such as protein concentration, heating time, temperature, salt concentration, seeding and stirring. ${ }^{14,41-43}$

Protein fibrils have also been discovered as a functional material in other fields apart from the foods. They have been used to prepare hybrids together with carbon nanomaterials and the recent development in this area has been reviewed by Li and Mezzenga (2013). ${ }^{44}$ For example, a pH-responsive hydrogel composed of $\beta$-lg fibrils and multiwalled carbon nanotubes is prepared and exhibits applications in biological fields, drug release, sensors and tissue engineering. ${ }^{45}$ Recently, Bolisetty and Mezzenga (2016) ${ }^{46}$ prepared a hybrid 
membrane using $\beta$-lg fibrils and activated porous carbon, which shows application opportunities in wastewater purification.

The protein fibrils used in this thesis are prepared from whey protein isolate (WPI) upon prolonged heating at $\mathrm{pH} 2$ at $80^{\circ} \mathrm{C}$ at low ionic strength. The reason of using whey protein isolate is that whey proteins are an important ingredient in food industry, because of their nutritional and functional properties. ${ }^{3-5}$ The reason why WPI-protein fibrils are used in this study is that they have been studied extensively ${ }^{2,47,48}$ and that these particular fibrils have a persistence length in the order of micrometers, ${ }^{17,18}$ which is comparable to the scale of emulsion droplets or other food relevant spherical particles. It is expected that this leads to interesting phase behaviour of fibril-sphere mixtures.

\subsubsection{Bacterial cellulose}

Cellulose is the most abundant biopolymer in nature and is used in many different areas. ${ }^{49,}$ ${ }^{50}$ Cellulose can be obtained from different sources, such as wood, plant and bacteria. ${ }^{50}$ Bacterial cellulose (BC), produced by certain types of bacteria, such as, Acetobacter xylinum, is a linear chain polysaccharide. ${ }^{51}$ The $B C$ microfibrils are $\sim 60 \mathrm{~nm}$ wide, $\sim 9 \mathrm{~nm}$ thick and up to several micrometers long. ${ }^{51} \mathrm{BC}$ has the same chemical structure as cellulose derived from plants. However, $\mathrm{BC}$ has a high purity without the lignin, pectin and hemicellulose present in plant cellulose. ${ }^{52}$ The hydroxyl group is the only functional group of BC. BC has attracted attention in different fields, such as biomedical and material science, cosmetics and paper-making. Much research has been focused on using BC in biomedical industry, ranging from tissue engineering, drug delivery to wound-dressing materials, with many examples that can be found in several review papers. ${ }^{53-55}$ In material science, $\mathrm{BC}$ has also been discovered to use in the development of composite materials for a variety of industrial applications. ${ }^{56-58}$ In paper-making industry, BC-based paper has shown various novel characteristics and offers various functionalities. ${ }^{59-61}$ In cosmetics industry, BC offers advantages such as stabilizing, rheology modifying and a high degree of hydration. ${ }^{62,63}$ More detailed research about the application of $B C$ in different fields can be found in many other review papers. ${ }^{52,55,63-69}$

$B C$ has also attracted increased attention of the food industry both due to its functional properties and health benefits. ${ }^{70} \mathrm{BC}$ forms a range of shapes and textures under certain conditions. $^{70}$ The functionality of $\mathrm{BC}$ in foods includes thickening, gelling, stabilizing and 
water binding ability. ${ }^{71-77}$ They have also been used as foaming and emulsifying agents. ${ }^{78-80}$ $B C$ is a dietary fibre and a low-calorie ingredient. ${ }^{71,76,77}$ One can already find its use in foods, i.e. the well-known dessert 'Nata de Coco', produced by the strain of Gluconacetobacter xylinus, consists of mainly the bacterial cellulose and water. ${ }^{81}$ In a recent study, a $B C$ microfibril dispersion was prepared by extracting the $B C$ microfibrils from the commercially available Nata de Coco through a high energy de-agglomeration process. $^{82}$ The microfibrils are present in the form of ribbon-shaped microfibrils with elementary fibrils acting as the building blocks. The width of the elementary fibrils is about $3 \mathrm{~nm}^{82}$ The resulting microfibrils are flexible. The average width is determined to be 60 $\mathrm{nm}$ and the average length is larger than $10 \mu \mathrm{m}$, which is too long to be measured from electron microscopy. The dispersion was found to remain highly heterogeneous, consisting of fibril bundles, flocs and voids due to strong attractions between the microfibrils. Upon increasing the BC microfibril concentration, the size of the flocs increases and the size of the voids decreases. Above a certain concentration, the $B C$ microfibrils form a space-filling network and show a gel-like behaviour. ${ }^{82}$ By adding carboxymethyl cellulose (CMC) during the de-agglomeration process, a more homogeneous dispersion of BC microfibrils was obtained, due to the adsorption of CMC on the $\mathrm{BC}$ microfibrils. However, structural heterogeneity on a macro-scale still remained due to incomplete mechanical de-agglomeration. ${ }^{83-85}$

In this thesis, we use BC microfibrils extracted from commercially available Nata de Coco through a high energy de-agglomeration process, as described in literature. ${ }^{82}$ It is of interest to study the value of $B C$ microfibrils, as a functional ingredient in foods, not only because of its multi-functionality, but also because it is natural, biodegradable, biocompatible and sustainable. ${ }^{63}$ Due to its high purity it serves as a model system for plant- and wood-derived cellulose. ${ }^{52}$

\subsubsection{Description of main systems}

In this thesis, four different mixed systems were investigated:

\subsubsection{WPI and BC microfibrils}

Different $\mathrm{pH}^{\prime} \mathrm{s}$ and concentration ratios are studied and results are presented in Chapter 2, 3, 4 and 5 . As described above, the BC microfibril dispersion prepared by high-energy de- 
agglomeration from Nata de Coco remain highly heterogeneous. Adding CMC during deagglomeration is found to alter the rheology and microstructure of the $\mathrm{BC}$ microfibrils, and thereby result in a more homogeneous dispersion. The aim of the study of WPI-BC microfibrils is to investigate whether WPI and the aggregation upon heating influence the rheology and microstructure of the $\mathrm{BC}$ microfibril dispersions, and vice versa, whether the $B C$ microfibrils influence the WPI denaturation and aggregation upon heating. The $\mathrm{pH}$ is adjusted to both above and below the iso-electric point (pl) of WPI, so the influence of charge reversal of the protein could be studied. The concentration of WPI both below and above their critical gelling concentration upon heating is studied in order to investigate the behaviour with BC microfibrils both in liquid and gelled state.

\subsubsection{WPI fibrils in emulsions and polystyrene latex dispersions}

The behaviour of WPI fibrils in dispersions containing emulsions and polystyrene latex dispersions are presented in Chapter 6 and 7. The addition of polymers to colloidal dispersions containing spheres is known to affect their stability and has received extensive attention in literature. ${ }^{86-88}$ Rod-like particles, for example, have shown to induce rich phase behaviour when added to colloidal dispersions containing spheres. ${ }^{89-94}$ However, the effect of WPI fibrils on the stability of colloidal dispersion containing spheres is not fully understood. ${ }^{29}$ Emulsions are thermodynamically unstable systems and polymers are often added to adjust the emulsions properties. ${ }^{95}$ The effect of WPI fibrils on the stability of WPI-stabilized o/w emulsions is studied at $\mathrm{pH} \mathrm{2,} \mathrm{in} \mathrm{which} \mathrm{both} \mathrm{the} \mathrm{WPI} \mathrm{fibrils} \mathrm{and} \mathrm{oil}$ droplets (WPI-stabilized) are positively charged. A range of WPI fibrils at different concentrations is added and the length of WPI fibrils is also changed. Furthermore, the study is extended to a model system containing negatively charged monodispersed particles, i.e. a polystyrene latex dispersion, where the phase behaviour becomes even more complex due to the charge difference between the latex particles and WPI fibrils.

\subsubsection{BC microfibrils in emulsions}

The stability of emulsions with addition of BC microfibrils is reported in Chapter 8 . The stabilization mechanisms of polysaccahrides include, for example, a viscosity increase of the continuous phase or gelation of the continuous phase. ${ }^{96} \mathrm{BC}$ microfibrils have also been used as a stabilizer in model food systems and emulsions with a pre-treatment of $\mathrm{BC}$ 
dispersions that is often different. ${ }^{72,78,79}$ The effect of BC microfibrils prepared by high energy de-agglomeration process on emulsion stability, however, has not yet received a systematic and detailed study.

\subsubsection{Bi-fibrillar networks in emulsions}

In Chapter 8, the effect of the bi-fibrillar network consisting of WPI fibrils and BC microfibrils on the stability of emulsions is studied. These studies are a continuation of the previous findings on a) the behaviour of mixtures containing both the WPI fibrils and BC microfibrils (Chapter 3), b) the effect of WPI fibrils on emulsion stability (Chapter 6), and c) the effect of $B C$ microfibrils on emulsion stability (Chapter 8). Using the results in Chapter 3, 6 and 8, the interdependencies between the various components in the system of bifibrillar networks in emulsions can now be identified.

\subsection{Discussion of the main findings}

\subsubsection{Behaviour of WPI and BC microfibrils}

In Chapter 2, 3, 4 and 5, the behaviour of mixtures containing WPI and BC microfibrils under different conditions are investigated. By varying the concentration ratios, $\mathrm{pH}, \mathrm{NaCl}$ concentration and further applying heating treatment, their physico-chemical properties in mixed solutions, mixed solutions after heating and further heat-induced mixed gels are investigated and characterized at both $\mathrm{pH} 2$ and $\mathrm{pH}$ 7. In general, both mixing WPI and $\mathrm{BC}$ microfibrils without heating and subsequently applying heating treatment lead to stable and homogeneous mixtures at $\mathrm{pH} \mathrm{7,} \mathrm{as} \mathrm{long} \mathrm{as} \mathrm{BC}$ microfibril concentration is above a critical value. This is because the $\mathrm{BC}$ microfibrils form a self-supporting gel and/or the viscosity increase from the WPI is sufficient to prevent the sedimentation of $B C$ microfibrils. The rheological properties of the mixture in dispersions were mainly dominated by the $\mathrm{BC}$ microfibrils, where two-shear thinning region and a viscosity plateau in between were observed. Upon a reversal of shear rate, a lower viscosity (also known as hysteresis) of the mixtures was observed, as shown in Figure 2a. However, this viscosity plateau and the hysteresis upon the reversal of shear rate were much less pronounced in the mixtures after heating above a certain WPI concentration (Figure 2b), whereas in pure $B C$ microfibrils these effects were still present. Microscopic images showed that the WPI aggregates and BC microfibrils co-existed in the system. WPI denatured and aggregated in 
the mixture in the same way as when it is heated alone. Upon gelation, the WPI and BC microfibrils form a duplex gel consisting of two independent and homogeneous networks spanning the whole system. The rheological, fracture properties and water holding capacity of the duplex gels are mainly controlled by the WPI gel. The presence of BC microfibrils increase the storage and loss modulus of the WPI gel up to a certain limit. When the strength of the WPI gel is high enough (by the addition of $\mathrm{NaCl}$ concentration), the influence of the BC microfibrils is negligible. The gelation of WPI in the mixtures is the same compared to as it is gelled alone. Increasing the $\mathrm{NaCl}$ concentration only alters the properties of the WPI gel, whereas BC microfibrillar gel is not affected.

At $\mathrm{pH} 2$, the WPI and BC microfibrils also form stable and homogeneous mixtures in the liquid state, both before and after heating. Despite the lower WPI concentration and the longer heating time ( 22 hours), the stability and rheological properties of the mixtures followed the same trend as the mixtures in $\mathrm{pH} 7$ (Figure 3a). However, the viscosity plateau and hysteresis are not observed after 22 hours heating at $\mathrm{pH} 2$ (Figure $3 \mathrm{~b}$ ). The rheology and microstructure of the pure BC microfibrils were not affected by prolonged heating at $\mathrm{pH}$ 2. One should note that at $\mathrm{pH} 2$ upon prolonged heating, WPI forms fibrils, which is a different process from that of $\mathrm{pH} 7$ upon heating for $30 \mathrm{~min}$, as described in the introduction. The presence of BC microfibrils resulted in formation of shorter WPI fibrils, although the length of WPI fibrils is not further quantified. The rheological properties of the mixtures were, however, not altered significantly with the presence of BC microfibrils. Microscopy images showed two fibrillar structures that are uniformly and independently present. Upon gelation at higher WPI concentration, a bi-fibrillar gel is formed consisting of a WPI fibrilllar gel and BC microfibrillar gel that co-exist. The presence of BC microfibrils increased the storage and loss modulus of the bi-fibrillar gel, while the ratio between the loss and storage modulus remained constant with an increase in BC microfibril concentration. The $\mathrm{BC}$ microfibrils did not influence the gelation dynamics and resulting overall structure of the WPI fibrillar gel. However, the WPI fibrils formed close to the BC microfibrils are more aligned than the fibrils formed further away from BC microfibrils. It seemed that BC microfibrils direct the growth of the WPI fibrils. The rheological and the fracture properties are mainly controlled by the properties of the WPI fibrillar gel.

The mixing of WPI and BC microfibrils did not only lead to bi-continuous dispersions in the liquid state, but also to bi-continuous gels (i.e. duplex gels). The WPI and BC microfibrils 
mixtures behave in the same way, as they are present alone. There interaction seems to be governed by their excluded volume. Bi-continuous protein-polysaccharide solutions and/or gels are rarely described in literature. Due to the thermodynamic incompatibility of proteins and polysaccharides, phase separation is more commonly observed. ${ }^{97-100}$ However, in biopolymer mixtures without salt where only one of the biopolymers is charged, phase separation is often suppressed since there is a large entropic penalty of segregating the co-ions. ${ }^{101,102}$ As a result, upon gelling, interpenetrating networks can be formed. ${ }^{103}$ However, increasing the salt concentration will promote the phase separation as the salt ions reduce the impact of the increased entropic term. ${ }^{101}$ The absence of phase separation in the WPI-BC microfibril mixtures studied is in line with the fact that only one biopolymer (i.e. WPI) is charged. Although literature reported that $\mathrm{BC}$ microfibrils have a streaming potential of $-7.5 \mathrm{mV},{ }^{104}$ because of the lack of charged groups in the $B C$ microfibrils, it is not expected that charge plays an important role in the behaviour of WPIBC microfibril mixtures studied here.

In an earlier study the adsorption of CMC on BC microfibrils was reported. The CMC enhances the distribution of the microfibrils leading to a more homogeneous $B C$ dispersion. ${ }^{83}$ To check whether WPI also adsorbs on the BC microfibrils, the WPI concentration in the supernatant of the mixtures (containing $3 \mathrm{wt} \% \mathrm{WPI}$ and $0.1 \mathrm{wt} \% \mathrm{BC}$ microfibrils) was measured by UV-absorbance after centrifugation. The absorbance did not decrease significantly in comparison to the WPI concentration in supernatant of a pure WPI (solution after centrifugation. This was investigated at $\mathrm{pH} 2, \mathrm{pH} 5$ and $\mathrm{pH} 7$ and $\mathrm{NaCl}$ concentrations from $0,25,50,100$ and $250 \mathrm{mM}$ using the same mixture. None of the samples showed measurable adsorption of WPI protein on the surface of BC microfibrils. We conclude that, within the sensitivity of the UV absorbance measurement, the WPI does not absorb on the BC microfibrils.

It was reported that at most $5 \% \mathrm{CMC}$ is closely associated with the $\mathrm{BC}$ micrifobrils, while the rest remains dissolved in solution. ${ }^{105}$ Although the amount of $\mathrm{CMC}$ absorbed on $\mathrm{BC}$ is reported to be relatively low, it is enough to have a significant influence on the microstructure of the BC microfibrils. In our case, no difference of BC microfibrils was observed in the mixtures with WPI. It is noted that the magnification used in our study for imaging is quite high in comparison to the images in the study of $\mathrm{BC}-\mathrm{CMC}^{83,84}$ Under the magnification used here, all BC microfibril structures look the similar. Heating the WPI-BC 
microfibril mixtures did modify the rheology properties. At $\mathrm{pH} 7$, when 9 wt\% WPI is present, the viscosity plateau and the hysteresis upon the reversal of shear rate (cf. Figure $2 \mathrm{~b}$ ) was much less pronounced in the mixtures after being heated. At $\mathrm{pH} \mathrm{2,} \mathrm{the} \mathrm{viscosity}$ plateau and hysteresis are absent after prolonged heating where WPI fibrils are formed in the mixtures (Figure $3 b$ ). It is noted that the way of sample preparation also affects the properties of the mixtures, as discussed in Chapter 2 and 3.
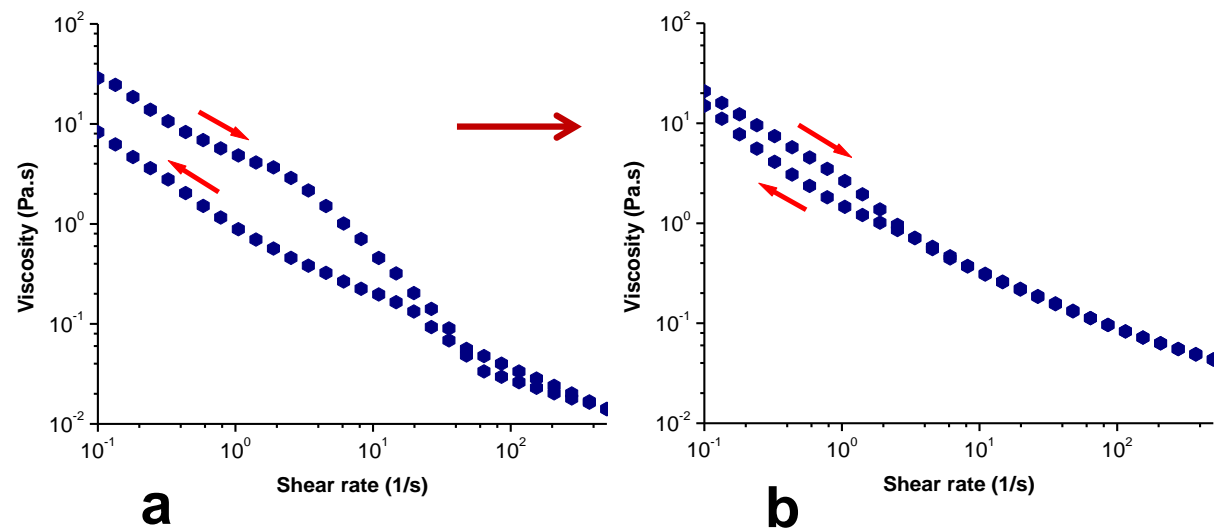

Figure 2 A typical flow curve of WPI-BC microfibril mixtures at $\mathrm{pH} 7$ before (a) and after (b) heating at $80{ }^{\circ} \mathrm{C}$ for $30 \mathrm{~min}$. The shear rate is first increased from 0.1 to $5001 / \mathrm{s}$ and subsequently decreased from 500 to $0.11 / \mathrm{s}$. 

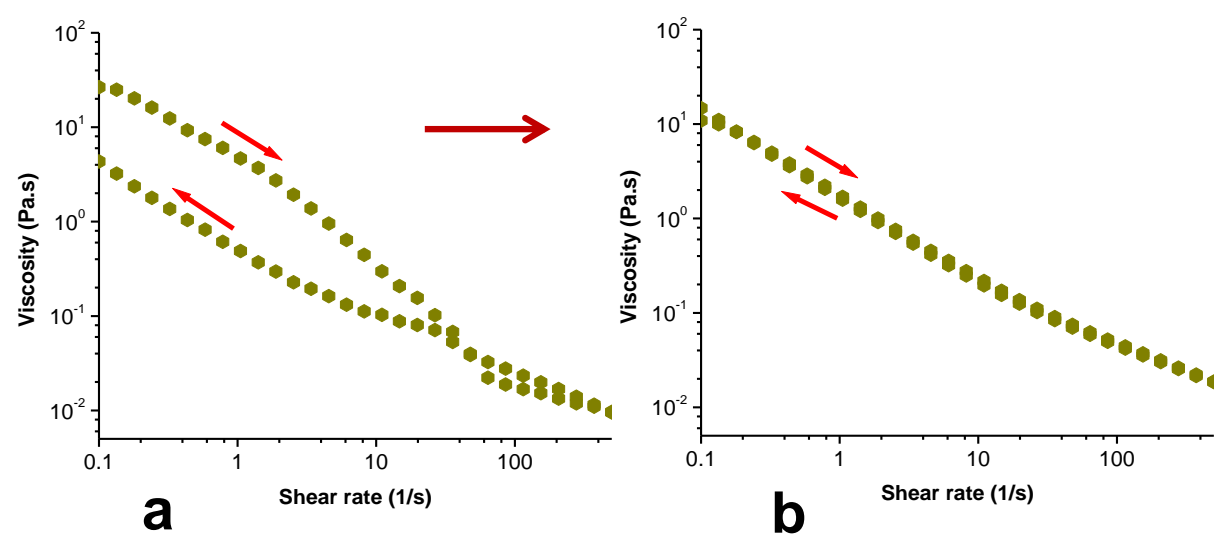

Figure 3 A typical flow curve of WPI-BC microfibril mixtures at pH 2 before (a) and after (b) heating at $80{ }^{\circ} \mathrm{C}$ for 22 hours. The shear rate is first increased from 0.1 to $5001 / \mathrm{s}$ and subsequently decreased from 500 to $0.11 / \mathrm{s}$.

\subsubsection{WPI fibrils in emulsions and polystyrene latex dispersions}

In Chapter 6 and 7, the behaviour of WPI fibrils at pH 2 in dispersions containing spheres, i.e. emulsions and polystyrene latex dispersions are studied. When WPI and spheres are both positively charged (i.e. WPI-stabilized emulsion), we observed depletion flocculation and depletion stabilization when the WPI fibril concentration increases. When WPI and the spheres are oppositely charged (i.e. polystyrene latex dispersions), bridging flocculation and steric/electrostatic stabilization were observed at low WPI fibril concentration, followed by depletion flocculation and depletion stabilization upon increasing WPI fibril concentrations (Chapter 7). It is known that not all hydrolysed peptides will be incorporated into the WPI fibrils; therefore, there are also nonaggregated/non-converted peptides present in the WPI fibril solution. To check whether the non-converted peptides play a role in the stability of the dispersions, the WPI fibrils were separated from the non-converted peptides using a centrifugal filter (Amicon Ultra 100K-15). Incubating them separately into the latex particle dispersions, followed by a washing step to remove the non-adsorbed WPI fibrils and non-converted peptides, it was found that both non-aggregated peptides and protein fibrils adsorbed on the surface of the latex particles. This was concluded from the reversal of the zeta potentials. The zeta 
potential value of the latex dispersion is around $-63 \mathrm{mV}$. The zeta potential of the latex dispersions incubated separately with pure WPI fibrils and pure non-converted peptides are around $+48 \mathrm{mV}$. This is the same zeta potential as the latex dispersion containing the mixture of fibrils and non-converted peptides. Since both of them can adsorb on the surface of the latex particles, it is unclear how much of the fibrils and non-converted peptides do adsorb. Earlier research showed that the non-aggregated peptides showed faster adsorption kinetics on both the water-oil and water-air surfaces in comparison to the fibrils. ${ }^{106-108}$ When mixing them separately into the latex dispersions and comparing to the stability of the latex dispersion containing the mixture of WPI fibrils and nonconverted peptides (Figure 4), one can observe that the latex dispersions containing pure WPI fibrils (Figure 4B) showed the same stability as the dispersions containing the mixture of WPI fibrils and non-converted peptides (Figure 4A). This clearly suggests that pure WPI fibrils are responsible for the bridging instability of the latex dispersions and not the nonconverted peptides. Although phase separation was also observed in some of the samples (Figure 4C), and microscopy images of the two phase separated samples from $A, B$ and $C$ showed the same aggregation behaviour (Figure 5), the effect of non-converted peptides is small in comparison to pure WPI fibrils. The phase separation in dispersions containing pure non-converted peptides could be due to the electrostatic attraction and/or limited bridging flocculation induced by the WPI fibrils that pass through the centrifugal filters (Figure 6). However, the amount of the WPI fibrils passing through the centrifugal filters is low as shown in Figure 6 to even zero as confirmed in literature. ${ }^{109}$ Overall, it can be concluded that the WPI fibrils are responsible for the behaviour of the polystyrene latex dispersions. 


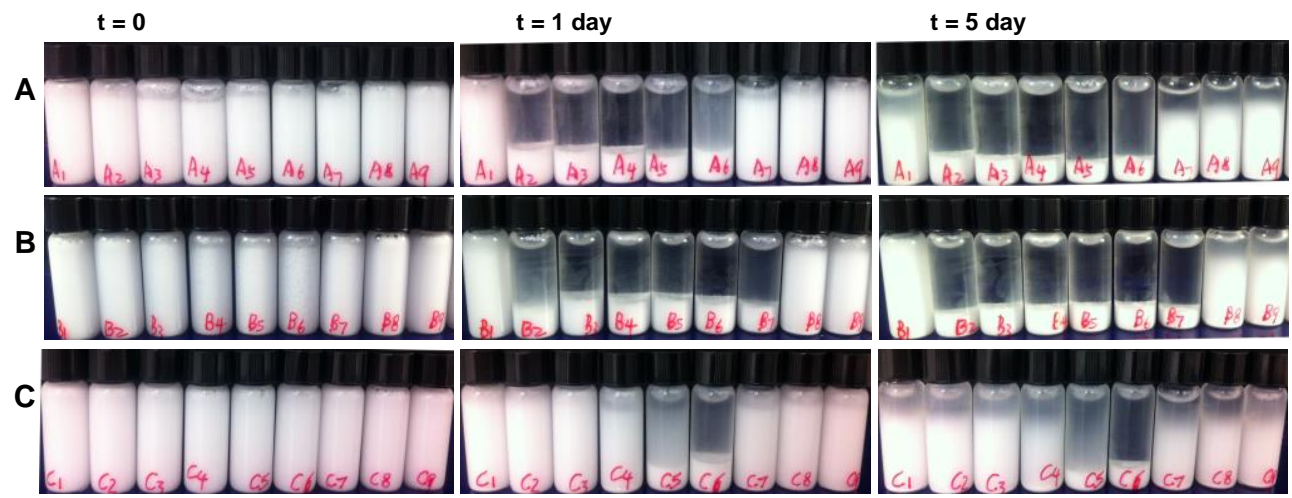

Figure 4 Images of 1 wt\% polystyrene latex dispersions with addition of mixture of WPI fibril and non-converted peptides (A), pure WPI fibrils (B) and pure non-converted peptides (C). The concentrations of $A, B$ and $C$ refer to the initial WPI concentration before centrifugation, which are: $0,0.001,0.0025,0.005,0.0075,0.01,0.025,0.05$, and $0.1 \mathrm{wt} \%$ corresponding to tubes from left to right. Images are taken at time 0,1 day and 5 days after sample preparation at $20^{\circ} \mathrm{C}$. 


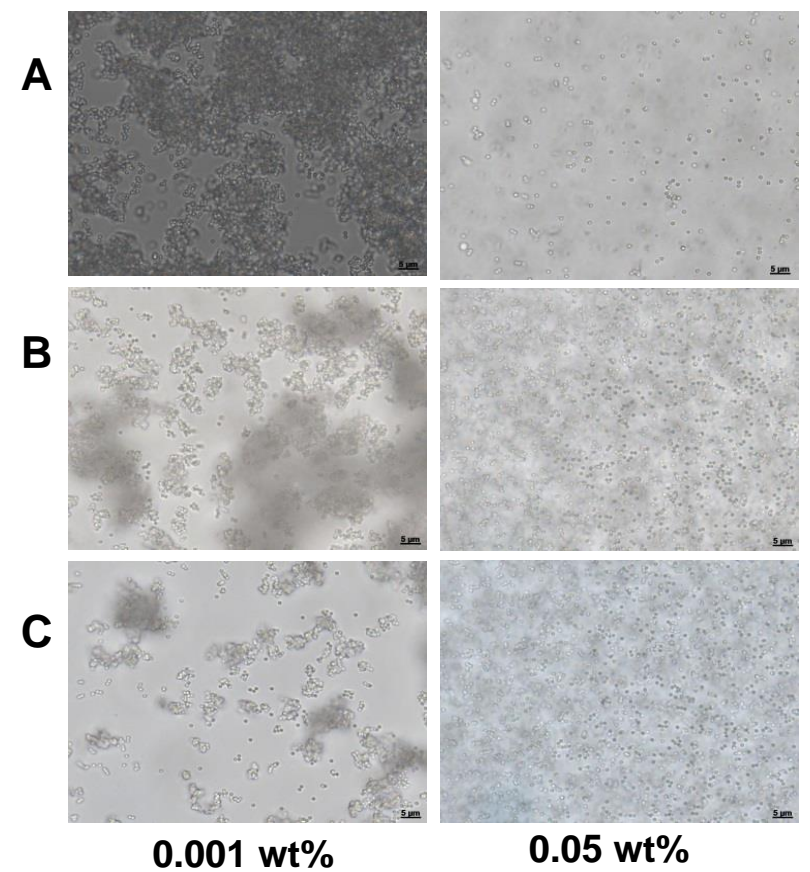

Figure 5 Microscopy images of 1 wt\% polystyrene latex dispersions with addition of mixture of WPI fibril and non-converted peptides (A), pure WPI fibrils (B) and pure non-converted peptides (C) at 7 days after sample preparation. The concentrations of $A, B$ and $C$ refer to the initial WPI concentration before centrifugation, which are: 0.001 and $0.05 \mathrm{wt} \%$. Scale bar is $1 \mu \mathrm{m}$.

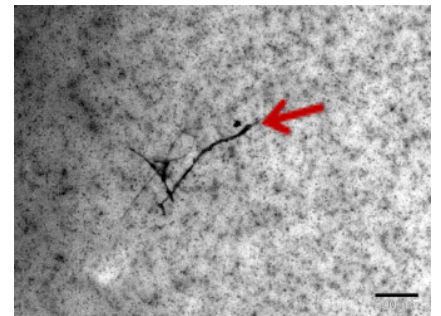

Filtrate 1

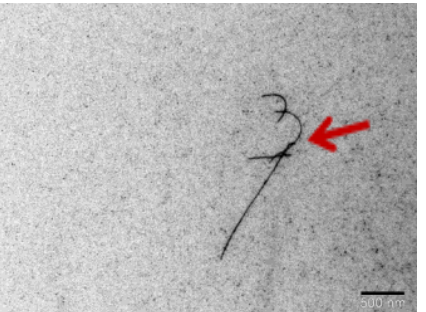

Filtrate 2

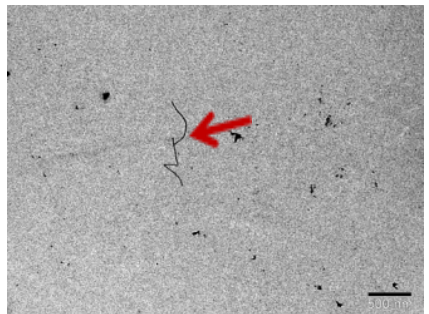

Filtrate 4

Figure 6 TEM images of the filtrate collected from the first, second and fourth centrifugation steps of separating the WPI fibrils from non-aggregated peptides using the centrifugal filters. Scale bar corresponds to $500 \mathrm{~nm}$. The red arrow indicates the fibrils. 
Above a certain WPI fibril concentration, stabilization in both emulsions and latex dispersions was observed. Microscopy images showed that both single particles/droplets and clusters of particles/droplets are present in the stabilized samples and the clusters of the particles do not grow beyond a certain size. No gelation of droplets/particles was observed and the viscosity of the both systems remained low, i.e. comparable to the viscosity of the pure WPI fibril solution. The stabilization is also found to be reversible, when diluted by the solvent and the WPI fibril dispersion at the same concentration (Chapter 6). This implies that the binding energy between the particles is in the order of thermal energy $k_{B} T$. The single particles and clusters of the particles are still relatively free to diffuse over a short length scale, but trapped on a larger length scale. The stabilization mechanism was attributed to the so-called depletion stabilization. ${ }^{110}$ In literature, the origin of depletion stabilization is still a subject of debate and the arguments exist between thermodynamic ${ }^{111-116}$ and kinetic effects. ${ }^{90,117-120}$ In the emulsions/dispersions stabilized by WPI fibrils, the stabilization points in the direction of depletion stabilization induced by a kinetic barrier. This kinetic barrier could be induced by the large amount of WPI fibrils that prevents the particle clusters from further approaching each other upon a certain size. Possible reasons could be the mesh size of WPI fibrils and, in addition, the fact that part of the WPI fibrils are in the nematic regime at the concentration that is able to stabilize the emulsions/ latex dispersions, may also play a role.

\subsubsection{Mesh size}

A cryo-SEM image from 4 wt\% WPI fibrils in Figure 7 shows a dense network composed of WPI fibrils. To calculate the mesh size $\xi$ of the WPI fibril network as a function of concentration, we assume that the WPI used in Chapter 6 and 7 contains 65 wt\% $\beta$ lactoglobulin and is the only protein involved in fibril formation under the conditions studied. ${ }^{15}$ In addition, the conversion rate of protein into fibrils was determined to be 58 $\%{ }^{14}$ As a result, the total amount of protein converted into fibrils is $38 \%$. The building blocks of the fibrils are peptides, ${ }^{16}$ thus, we assume that one protein provides one peptide for fibril formation. The volume of one peptide equals to $\frac{\pi}{4} D^{2} l$, where the diameter $D$ is 4 $\mathrm{nm}^{19}$ and the length $\mathrm{I}$ is $2.4 \mathrm{~nm} .{ }^{16}$ The mesh size $\xi$ of the fibril solutions for the various fibril concentrations was calculated (Table 1 ) based on: $:^{121-123}$ 


$$
\xi=\frac{1}{\sqrt{c_{L}}}
$$

Where $c_{L}$ is the length concentration of the fibrils.

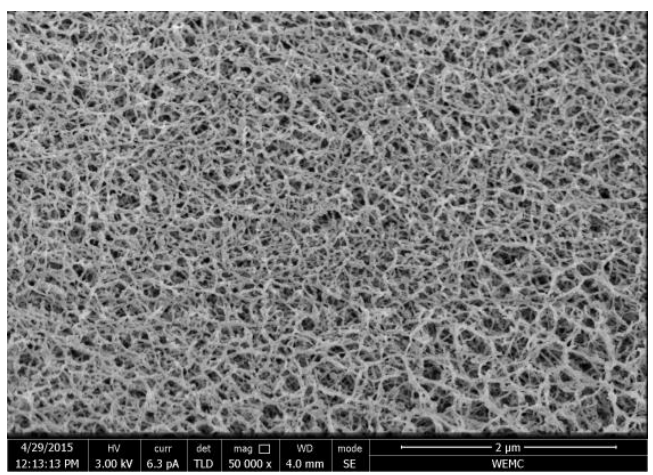

Figure 7 A cryo-SEM image of a 4 wt\% WPI fibril solution formed at $\mathrm{pH} 2$ upon heating at $80^{\circ} \mathrm{C}$ for 22 hours. Scale bar corresponds to $2 \mu \mathrm{m}$. 
Table 1 Mesh size $\xi$ calculated for various WPI concentrations or WPI fibril volume fractions $\Phi$.

\begin{tabular}{|c|c|c|}
\hline $\begin{array}{l}\text { WPI concentration } \\
\qquad(\mathbf{w t} \%)\end{array}$ & $\begin{array}{l}\text { Fibril volume fraction } \\
\qquad(\Phi)\end{array}$ & $\begin{array}{l}\text { Mesh size } \xi \\
\quad(\mathbf{n m})\end{array}$ \\
\hline 0.001 & $3.75 \times 10^{-6}$ & 1831 \\
\hline 0.0025 & $9.37 \times 10^{-6}$ & 1158 \\
\hline 0.005 & $1.87 \times 10^{-5}$ & 819 \\
\hline 0.0075 & $2.81 \times 10^{-5}$ & 668 \\
\hline 0.01 & $3.75 \times 10^{-5}$ & 579 \\
\hline 0.025 & $9.37 \times 10^{-5}$ & 366 \\
\hline 0.075 & $2.81 \times 10^{-4}$ & 211 \\
\hline 0.1 & $3.75 \times 10^{-4}$ & 183 \\
\hline 0.5 & $1.87 \times 10^{-3}$ & 82 \\
\hline 1 & $3.75 \times 10^{-3}$ & 58 \\
\hline 1.5 & $5.62 \times 10^{-3}$ & 47 \\
\hline 2 & $7.50 \times 10^{-3}$ & 41 \\
\hline 2.25 & $8.43 \times 10^{-3}$ & 39 \\
\hline 2.5 & $9.37 \times 10^{-3}$ & 37 \\
\hline 2.75 & $1.03 \times 10^{-2}$ & 35 \\
\hline 3 & $1.12 \times 10^{-2}$ & 33 \\
\hline 3.25 & $1.22 \times 10^{-2}$ & 32 \\
\hline 3.5 & $1.31 \times 10^{-2}$ & 31 \\
\hline 4 & $1.50 \times 10^{-2}$ & 29 \\
\hline
\end{tabular}

The mesh size of the WPI fibrils above $0.5 \mathrm{wt} \%$ is smaller than $100 \mathrm{~nm}$ (Table 1). This is smaller than the particle diameters we studied in emulsions $(\sim 2 \mu \mathrm{m})$ in Chapter 6 and latex dispersions (208 $\mathrm{nm}$ and $862 \mathrm{~nm}$ ) in Chapter 7. Therefore, it is possible that the small mesh size aids in preventing the particles/clusters of particles from further approaching, thus, kinetically stabilize the dispersions. 


\subsubsection{Nematic phase}

Another possible effect is that WPI fibrils show a transition from an isotropic phase (I) to a nematic phase $(\mathrm{N})$ above a certain fibril concentration. ${ }^{2,32}$ To predict the fibril concentration of this I-N transition, it is assumed that the WPI fibrils behave as rod-like particles. The prediction of this I-N transition in dispersions of rod-like particles was initially made by Onsager ${ }^{124}$ and later extended to charged and semi-flexible rods. ${ }^{125,126}$ The WPI fibrils are positively charged at $\mathrm{pH} 2$ and the critical volume fraction of fibrils at the I-N transition, $\phi^{*}$, is given by, ${ }^{33,127}$

$$
\phi^{*}=6 \frac{D^{2}}{D_{e f f} L_{p}}
$$

with $L_{p}$ the persistence length of fibrils $\left(L_{p}=1.6 \mu \mathrm{m}^{18}\right)$ and the effective diameter, $D_{\text {eff, }}$, is given by

$$
D_{\text {eff }}=D+\kappa^{-1}\left(\ln A+C+\ln 2-\frac{1}{2}\right)
$$

with $\mathrm{K}^{-1}$ the Debye electrostatic screening length, $C$ Eulers's constant $(C=0.577)$ and the constant $A$ is given by ${ }^{126}$

$$
A=2 \pi v_{e f f}^{2} \kappa^{-1} Q \exp (-\kappa D)
$$

where $v_{\text {eff }}$ denotes the effective linear charge density of the rod and $Q$ is the Bjerrum length.

The effective linear charge density $v_{\text {eff }}$ was calculated by using a charge per peptide of $+2 e$ $\left(\mathrm{e}=1.6 \times 10^{-19} \mathrm{C}\right)$. The Debye length $\mathrm{K}^{-1}$ is calculated from

$$
\kappa^{-1}=\sqrt{\frac{\varepsilon_{0} \varepsilon_{r} k_{B} T}{2 N_{A} e^{2} I}}
$$

with $\mathrm{N}_{\mathrm{A}}$ Avogadro's constant $\left(\mathrm{N}_{\mathrm{A}}=6.02 \times 10^{23}\right)$, e elementary charge $\left(\mathrm{e}=1.6 \times 10^{-19} \mathrm{C}\right), \quad \varepsilon_{0}$ dielectric permittivity in vacuum $\left(\varepsilon_{0}=8.85 \times 10^{-12} \mathrm{~F} / \mathrm{m}\right), \varepsilon_{\mathrm{r}}$ dielectric permittivity in water $\left(\varepsilon_{\mathrm{r}}\right.$ 
$=80.1), \mathrm{k}_{\mathrm{B}}$ Boltzmann's constant $\left(\mathrm{k}_{\mathrm{B}}=1.38 \times 10^{-23} \mathrm{~J} / \mathrm{K}\right), \mathrm{T}$ absolute temperature $(\mathrm{T}=293 \mathrm{~K})$, I the ionic strength in $M$.

The Bjerrum length $Q$ is calculated as follows:

$$
Q=\frac{e^{2}}{4 \pi \varepsilon_{0} \varepsilon_{r} k_{B} T}
$$

For our experimental condition of $\mathrm{I}=0.01 \mathrm{M}$, we find $\phi^{*}=6.52 \times 10^{-3}$. This value would correspond to a WPI concentration of $1.5 \mathrm{wt} \%$ (Table 1), implying a fibril concentration of $0.57 \mathrm{wt} \%$ (assuming a conversion of $38 \mathrm{wt} \%$ protein to fibrils). This fibril concentration of 0.57 wt\% is satisfactory in comparison to the theoretical value of $0.24 \%$, given by Mezzenga et al.. ${ }^{33}$ Experimentally, the I-N transition of the $\beta$-lg fibrils was found to occur between $0.4 \%-0.5 \%{ }^{18,32,33}$ In our studies, the depletion stabilization as induced by the WPI fibrils in the emulsions and latex dispersions were found to occur around $2.5 \mathrm{wt} \%$ (initial WPI concentration), i.e. $\phi \sim 0.01$ protein fibrils. This means that the depletion stabilization induced by WPI fibrils takes place above the I-N transition, $\phi=0.0065$. However, the nematic phase will exists in domains surrounded by an isotropic phase as long as the volume fraction of fibrils is below the single nematic phase transition at $\phi=0.027 .{ }^{18,128}$ Since for the depletion stabilisation concentration we have $\phi=0.01$, i.e. outside of the single nematic phase, we have nematic domains present in a isotropic phase. ${ }^{18,128}$ Therefore the isotropic phase will play a role in determining the stability, and it will not be due to the presence of a nematic phase only. Apart from this, the nematic phase will still provide a kinetic barrier similar to that of an isotropic phase. The presence of clusters of particles of a finite size in the depletion stabilized samples reveals the occurrence of aggregation between the particles. We attribute this aggregation to the depletion interaction. These clusters are reversible upon dilution (either in the fibril phase or the aqueous phase). The fact that the clusters have a maximum size indicates that there is a mechanism that acts against aggregation of larger clusters. We propose that this is due to a kinetic barrier as induced by the network formed by the fibrils. The fact that one observes clusters and single droplets is proposed to be caused by the fact that the single particles are in kinetic equilibrium with the clusters. 


\subsubsection{BC microfibrils and WPI-BC bi-fibrillar network in emulsions}

Above a certain concentration the BC microfibrils form a self-supporting network. ${ }^{82}$ This network is able to prevent the oil droplets from creaming thereby stabilizing the emulsion, as demonstrated in Chapter 8. WPI fibrils and BC microfibrils form stable and homogeneous mixtures, as presented in Chapter 3. When adding them together to emulsions, the two fibrillar structures affect the stabilisation of the emulsions. For both fibrillar structures, at low concentrations, the emulsions are destabilized. Above a certain WPI fibril concentration, depletion flocculation induced by WPI fibrils leads to enhanced creaming. However, this enhanced creaming can be prevented when the BC microfibril concentration is high enough. In this case a network is formed with a yield stress that is high enough to stop the creaming of oil droplets. At sufficiently high WPI fibril concentration, the emulsions can be stabilized by a mechanism referred to as depletion stabilization, where both single droplets and small droplet clusters are present. As a result, the competition between the destabilization and stabilization of emulsions induced by WPI fibrils and BC microfibrils leads to different microstructures. The results in Chapters 6 , 7 and 8 , on the stability of emulsions mixed with fibrillar structures are schematically summarized in Figure 8. The different stabilization and destabilization mechanisms of WPI fibrils and $B C$ microfibrils lead to different emulsions with different microstructures and rheological properties. Figure 8 only shows the influence of the concentration of the fibrillar structures on the emulsions. However, other parameters, such as, length distribution of the fibrils, size distribution and charge of the oil droplets, $\mathrm{pH}$, ionic strength and temperature also have an influence on the emulsion stability. 


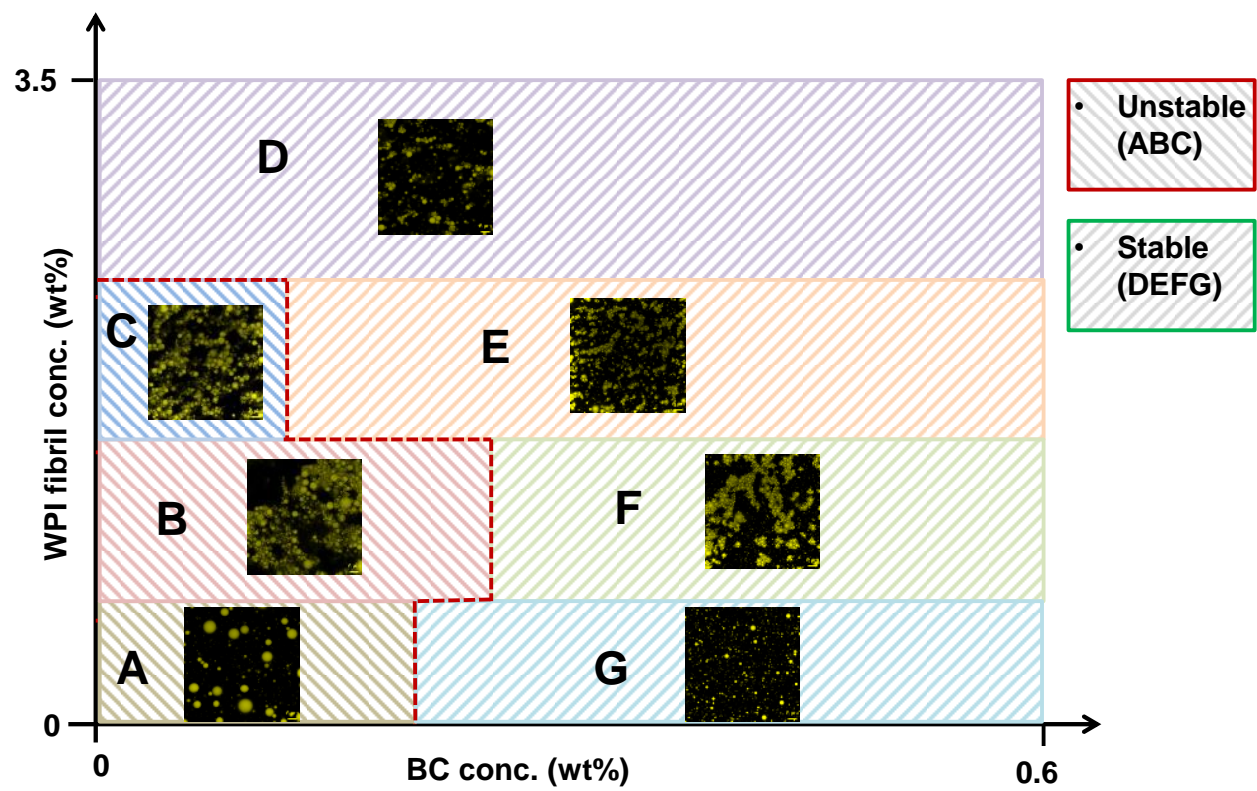

Figure 8 The stability diagram of emulsions containing bi-fibrillar networks consisting of WPI fibrils and $B C$ microfibrils at different concentrations. The $A, B$ and $C$ areas correspond to emulsions that are unstable. The areas of D, E, F, and G correspond to stable emulsions. The CLSM image in each area shows the typical microstructure of the emulsions.

\subsubsection{Properties of the WPI fibrils and BC microfibrils}

Apart from the concentration of WPI fibrils (Chapter 6), their average length also influences their efficiency to stabilize or to destabilize an emulsion. It is known that the

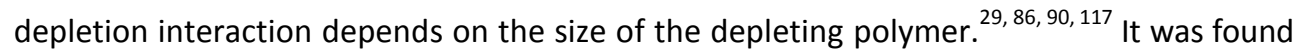
that longer fibrils are more efficient in emulsion stabilization. In the case of BC microfibrils, previous research has shown that the variation in the high energy de-agglomeration process (such as homogenization pressure and the number of passes through the homogenizer) influences the microstructure of the microfibrils (i.e. the size of the flocs, microfibril bundles and voids in the dispersions). ${ }^{82,129}$ Based on these results, it is clear that the pre-treatment used will have an influence on the effect of $B C$ microfibrils on emulsion stability. For instance, the stabilization of emulsions may occur at different BC microfibril concentrations depending on the pre-treatment used. ${ }^{75}$ 


\subsubsection{Properties of the dispersed spheres}

In Chapter 6, the effect of the volume fraction of the oil droplets on emulsion stability with addition of WPI fibrils was studied. It was found that the volume fraction only has a small influence. In Chapter 7, negatively charged latex dispersions in the presence of positively charged WPI fibrils were studied. This led to a different behaviour compared to the emulsions where the WPI fibrils and the oil droplets are both positively charged. Due to the adsorption of the positively charged WPI fibrils on the negatively charged latex spheres, we observed bridging flocculation. ${ }^{130}$ Further increasing the WPI fibril concentration led to steric and/or electrostatic stabilization, ${ }^{131}$ followed by first depletion flocculation and then depletion stabilization. When the diameter of the latex particles becomes smaller, a higher WPI fibril concentration is needed to stabilize the dispersion against bridging. This is because at the same weight concentration of particles, the specific area of the particles increases with decreasing particle diameter.

\subsubsection{External parameters: temperature, salt concentration and $\mathrm{pH}$}

\subsubsection{Temperature}

Parameters, such as temperature, ionic strength and $\mathrm{pH}$ play an important role in the stability of dispersions, and as a consequence, also in many food products. Both WPI fibrils and $\mathrm{BC}$ microfibrils were found to be able to stabilize the emulsions, however, it is also important to know how stable the emulsions are against changes in temperature, ionic strength and $\mathrm{pH}$.

In Chapters 6, 7 and 8, the effect of temperature on dispersions stabilized by fibrillar structures was investigated. The stabilization by fibrillar structures was found to be basically temperature independent. It was postulated that the stabilization by WPI fibrils has an entropic origin. The network of the BC microfibrils and the resulting yield stress are not influenced by temperature as found in Chapters 2 and 3. Emulsions mixed with BC microfibrils also remain stable against heating, which is in line with other research. ${ }^{72,78}$ Not surprisingly, the rheological properties of micro-fibrillated wood and plant cellulose were also found to be independent of temperature up to $80^{\circ} \mathrm{C} .^{132-134}$ 


\subsubsection{NaCl concentration}

In Chapter $8, \mathrm{NaCl}$ is added to the emulsions stabilized by only BC microfibrils and a WPIBC bi-fibrillar network. In case of emulsions stabilized only by BC microfibrils the macroscopic stability was also not influenced by $\mathrm{NaCl}$ up to concentrations of $150 \mathrm{mM}$ $\mathrm{NaCl}$. The emulsions stabilized by WPI fibrils and BC microfibrils showed enhanced macroscopic stability (i.e. delayed creaming), when $150 \mathrm{mM} \mathrm{NaCl}$ was added. The microstructure of $\mathrm{BC}$ microfibrils is found to be unaffected by salt addition. ${ }^{78}$ This explains why emulsions with $150 \mathrm{mM} \mathrm{NaCl}$ can be stabilized by BC microfibrils. Both WPI fibrils and oil droplets are positively charged and adding $\mathrm{NaCl}$ will screen their charges, facilitating the aggregation. Bolisetty et al. ${ }^{135}$ studied the phase behaviour of $\beta$-lg fibrils at different ionic strengths. They found that increasing the $\mathrm{NaCl}$ concentration in $\beta$-lg fibrils dispersions induces phase transitions from nematic phases to gelled phases. A transition to a translucent phase and a macroscopic phase separation can be induced by further increasing the $\mathrm{NaCl}$ concentration. As a result, the delayed creaming of emulsions containing both WPI fibrils and BC microfibrils at $150 \mathrm{mM}$ may be attributed to the increased viscosity of the WPI fibril network.

\subsubsection{3 $\mathrm{pH}$}

The acidity of food products is usually between $\mathrm{pH} 4$ and $\mathrm{pH} 7$. Our studies on emulsion stabilized by only WPI fibrils (Chapter 6 and 7), only BC microfibrils and WPI-BC bi-fibrillar mixtures (Chapter 8) are performed at $\mathrm{pH} 2$. For industrial applications, it is important to investigate how emulsion stabilization by only WPI fibrils (Chapter 6 and 7), only BC microfibrils and WPI-BC bifibrillar networks (Chapter 8 ) is influenced by a pH change.

When adjusting the $\mathrm{pH}$ of emulsions containing WPI fibrils at $\mathrm{pH} 2$ to $\mathrm{pH} 5$ or $\mathrm{pH} 8$, the stability of the emulsions was followed for 7 days as shown in Figure 9. A strain sweep was performed on an $10 \mathrm{wt} \% \mathrm{O} / \mathrm{W}$ emulsion containing $2.5 \mathrm{wt} \% \mathrm{WPI}$ fibrils at $\mathrm{pH} 2, \mathrm{pH} 5$ and $\mathrm{pH} 8$, as shown in Figure 10. The emulsion stability containing WPI fibrils at $\mathrm{pH} 2$ was discussed in Chapter 6. From the images (Figure 9), it is observed that at $\mathrm{pH} 5$, the emulsion without WPI fibrils is phase separated, with an layer of oil droplets on top and a serum layer at the bottom. At WPI fibril concentrations from 0.001 to $0.05 \mathrm{wt} \%$, the emulsions show a similar phase separation as the emulsion without WPI fibrils. By increasing the WPI fibrils concentration from 0.075 to $0.75 \mathrm{wt} \%$, the phase separation 
becomes less pronounced. At WPI fibril concentrations of $1 \mathrm{wt} \%$ and above, the emulsions remained stable against creaming. The storage modulus $G^{\prime}$ is higher than the loss modulus $\mathrm{G}^{\prime \prime}$, with values also higher than that for $\mathrm{pH} 2$ (Figure 10). This indicated that an emulsion gel was formed at a WPI fibril concentration of $2.5 \mathrm{wt} \%$. The emulsion at $\mathrm{pH} 8$ without fibrils creamed. At WPI fibril concentrations between 0.001 to 0.05 wt\%, a similar creaming behaviour was observed as the emulsion without WPI fibrils. Further increasing WPI fibril concentration from 0.075 to $0.1 \mathrm{wt} \%$, only limited creaming of emulsions were observed. At WPI fibril concentration at and above $0.5 \mathrm{wt} \%$, the emulsions remained stable for 7 days. The values of the storage modulus $G^{\prime}$ and loss modulus $G^{\prime \prime}$ in emulsion containing $2.5 \mathrm{wt} \% \mathrm{WPI}$ fibrils were comparable to the emulsion at $\mathrm{pH}$ 2. Both emulsions at $\mathrm{pH} 2$ and $\mathrm{pH} 8$ containing $2.5 \mathrm{wt} \%$ WPI fibrils have a liquid-like behaviour.

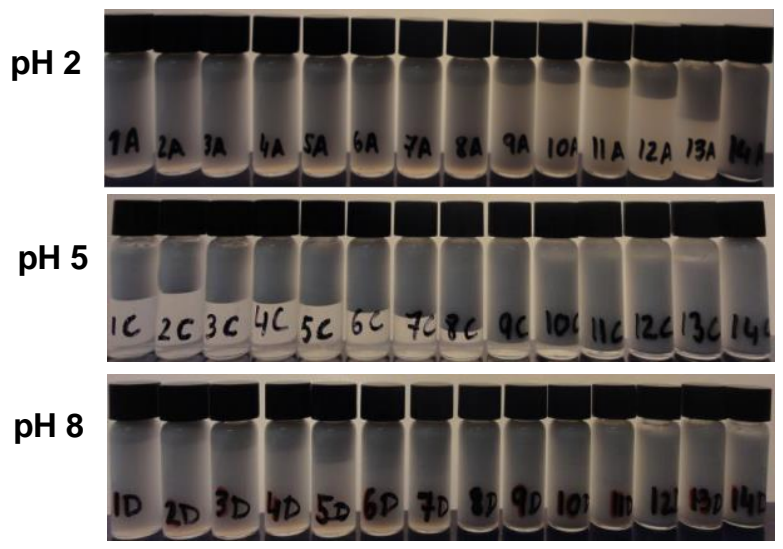

Figure 9 Images of 10 wt\% WPI-stabilized O/W emulsions with WPI fibrils. Samples were stored at $20^{\circ} \mathrm{C}$ for 7 days after preparation. The WPI fibril concentrations are: $0,0.005,0.01,0.025,0.05$, $0.075,0.1,0.25,0.5,0.75,1,1.5,2$ and $2.5 w t \%$, corresponding to the tubes from left to right. 


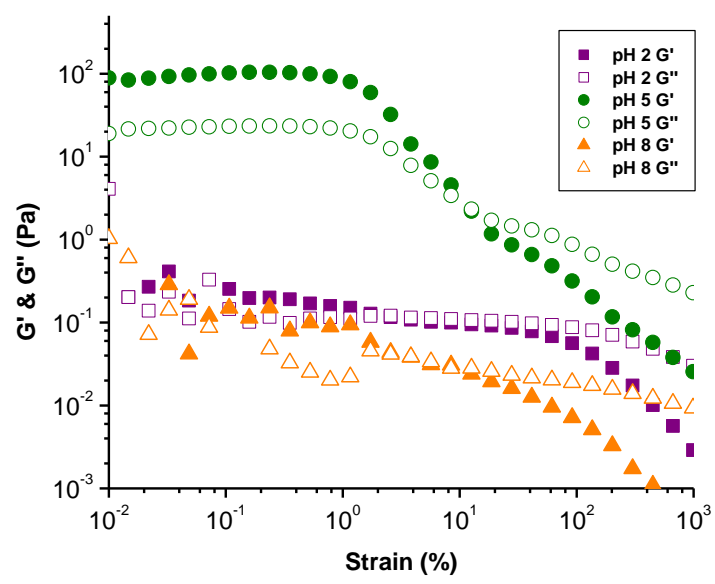

Figure 10 Storage modulus G' and loss modulus G" as a function of strain for $10 \mathrm{wt} \%$ emulsions containing 2.5 wt\% WPI fibrils at $\mathrm{pH} \mathrm{2,5}$ and 8.

When adjusting the $\mathrm{pH}$ of emulsions stabilized by 0.45 wt\% BC microfibrils from $\mathrm{pH} 2$ to $\mathrm{pH} 7$, the sample remained stable for 6 days (Figure 11). In emulsions containing both WPI fibrils and BC microfibrils at different concentrations, all emulsions remained stable for 6 days (Figure 11). Interestingly, the emulsions at pH 7 showed better stability, i.e. a slower creaming rate, compared to the emulsions at $\mathrm{pH} 2$ discussed in Chapter 8. This is in line with the results for the emulsions containing only WPI fibrils (Chapter 6).

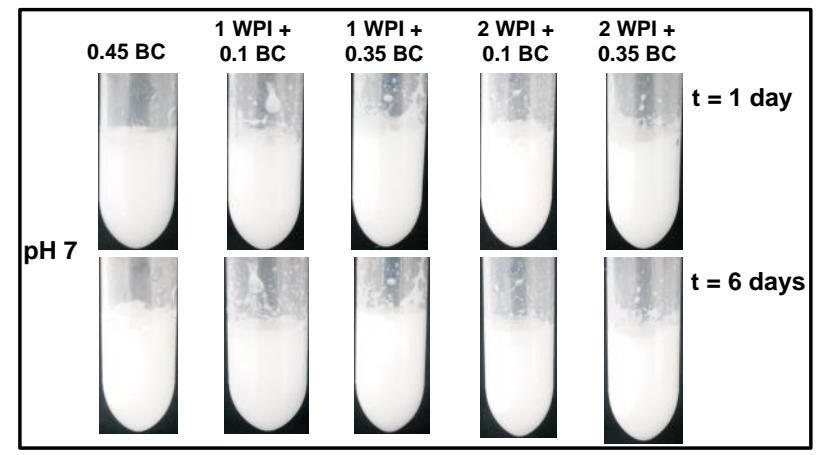

Figure 11 Images of a $10 \%(\mathrm{v} / \mathrm{v})$ emulsion containing BC microfibrils and/or WPI fibrils at pH 7 at 1 and 6 days after preparation. The concentration of WPI fibrils (wt\%) and BC microfibrils (wt\%) are indicated on the top of the image. 
The effect of $\mathrm{pH}$ on protein fibrils formed at $\mathrm{pH} 2$ has been studied by several authors. ${ }^{14}$ 107-109, 136-138 Bolder et al. $^{14}$ found that when WPI fibrils formed at $\mathrm{pH} 2$ were stored at $\mathrm{pH} 7$ overnight, the viscosity of the sample was increased. When stored at $\mathrm{pH} 10$ overnight, the samples were even gelled. They proposed that the viscosity increase is caused by the formation of disulphide bonds between non-assembled proteins, causing the formation of protein clusters. Jung et al. ${ }^{137}$ found that precipitation of a $\beta$-lg fibril solution (in the presence of non-aggregated peptides) occurred when the $\mathrm{pH}$ is adjusted to the isoelectric point of the protein. The WPI fibril solution contains both WPI fibrils and non-aggregated peptides. By using a centrifugal filter and separating the WPI fibrils from the nonaggregated peptides, Kroes et al. ${ }^{109}$ studied and compared the effect of $\mathrm{pH}$ on stability of pure WPI fibrils, pure non-aggregated peptides and mixture of WPI fibrils and nonaggregate peptides. They found that the mixtures of WPI fibrils and non-aggregated peptides at $\mathrm{pH} 2$ and 8 are transparent. At pH 5 the mixtures are turbid, which turned out to be mainly caused by the aggregation of the non-aggregated peptides. At $\mathrm{pH} 5$ the WPI fibrils tend to aggregate, since their net charge is close to zero. At $\mathrm{pH} 8$, the pure nonaggegrated peptides still form small aggregates, although much smaller than the aggregates formed at $\mathrm{pH} 5$.

In conclusion, it is obvious that the $\mathrm{pH}$ change influences both the rheological and microstructural properties of the WPI fibrils. Therefore, the changes in stability of emulsions as a function of $\mathrm{pH}$ may be explained by the change of WPI fibrils, in both emulsion containing only WPI fibrils and both WPI fibrils and BC microfibrils. The rheological properties and microstructure of BC microfibrils were not affected significantly by $\mathrm{pH}$, as shown in Chapter 2 and Chapter 3. In addition, emulsions stabilized by BC microfibrils were found to be stable against $\mathrm{pH}$ change. ${ }^{78}$

\subsection{Concluding remarks}

We find that the stabilization/destabilization mechanisms of WPI fibrils are different from those of BC microfibrils. We conclude that the WPI fibrils and BC microfibrils do not interfere with one another except via relatively weak excluded volume interactions. The BC microfibrils form a network that exhibits a yield stress. One is able to stabilise emulsions using WPI fibrils while retaining the emulsion fluidity. In addition one is able to add $\mathrm{BC}$ microfibrils to increase viscosity in an independent manner, i.e. without changing 
the microstructure of the emulsion. Therefore combining WPI fibrils and BC microfibrils makes it possible to tune emulsion properties in a more controlled manner when using both fibrillar structures compared to using only one of them.

One can think of emulsion properties like rheological properties and microstructural features. In addition, emulsions and latex dispersions stabilized by WPI fibrils through depletion stabilization are able to maintain the low viscosity of the fluid. The above findings suggest many applications in industry, ranging from fine-tuning thickening, gelling to stabilization. It also allows realizing multi-functionality in products, where texture and nutritional value are combined. The knowledge obtained can also be extended to other protein systems like plant proteins, that have received increased attention in literature in view of sustainability, availability and cost. ${ }^{139,140}$

Regarding the stabilizing effect in emulsions from both WPI fibrils and BC microfibrils, one can also extend to other colloidal systems. Furthermore, the depletion stabilization mechanism has unique advantages in comparison to other stabilization mechanisms such as steric stabilization and/or electrostatic stabilization. ${ }^{110}$ Indeed, by applying the depletion stabilization mechanism, colloidal stability in extremely high ionic strength, extreme $\mathrm{pH}$, organic solvents, heavy metals etc. was achieved, while still remaining surface accessibility. ${ }^{141}$ In addition, the viscosity of the system still remains low, as found in our studies.

The BC microfibrils prepared from high energy de-agglomeration have their advantage for use in foods as no chemical treatment is involved. However, the BC microfibrils also have disadvantages. One potential problem in applications namely is that the system still remains heterogeneous, ${ }^{82}$ which sometimes may require addition of dispersing agents. Therefore, research on this system still remains rather challenging and extra care should be taken for interpretation of data. 


\subsection{References}

1. E. Dickinson, Food Hydrocolloids, 2016, 52, 497-509.

2. A. Kroes-Nijboer, P. Venema and E. van der Linden, Food \& function, 2012, 3, 221-227.

3. J. De Wit, Journal of Dairy Science, 1998, 81, 597-608.

4. G. W. Smithers, International dairy journal, 2008, 18, 695-704.

5. C. V. Morr and E. Y. W. Ha, Critical Reviews in Food Science and Nutrition, 1993, 33, 431-476.

6. T. Nicolai, M. Britten and C. Schmitt, Food Hydrocolloids, 2011, 25, 1945-1962.

7. T. Nicolai and D. Durand, Current Opinion in Colloid \& Interface Science, 2013, 18, 249-256.

8. M. R. Krebs, G. L. Devlin and A. Donald, Biophysical Journal, 2007, 92, 1336-1342.

9. E. Bromley, M. Krebs and A. Donald, The European Physical Journal E, 2006, 21, 145-152.

10. S. Ikeda and V. J. Morris, Biomacromolecules, 2002, 3, 382-389.

11. A. Clark, G. Kavanagh and S. Ross-Murphy, Food Hydrocolloids, 2001, 15, 383-400.

12. E. A. Foegeding, E. L. Bowland and C. C. Hardin, Food Hydrocolloids, 1995, 9, 237-249.

13. E. A. Foegeding, J. P. Davis, D. Doucet and M. K. McGuffey, Trends in Food Science \& Technology, 2002, 13, 151-159.

14. S. G. Bolder, A. J. Vasbinder, L. M. C. Sagis and E. van der Linden, International dairy journal, 2007, 17, 846-853.

15. S. G. Bolder, H. Hendrickx, L. M. C. Sagis and E. van der Linden, Journal of Agricultural and Food Chemistry, 2006, 54, 4229-4234.

16. C. Akkermans, P. Venema, A. J. van der Goot, H. Gruppen, E. J. Bakx, R. M. Boom and E. van der Linden, Biomacromolecules, 2008, 9, 1474-1479.

17. E. H. C. Bromley, M. R. H. Krebs and A. M. Donald, Faraday Discussions, 2005, 128, 13-27.

18. L. M. C. Sagis, C. Veerman and E. van der Linden, Langmuir, 2004, 20, 924-927.

19. L. N. Arnaudov, R. de Vries, H. Ippel and C. P. M. van Mierlo, Biomacromolecules, 2003, 4, 16141622.

20. J. Adamcik, J.-M. Jung, J. Flakowski, P. De Los Rios, G. Dietler and R. Mezzenga, 2010, 5, 423428.

21. C. Akkermans, A. J. Van der Goot, P. Venema, E. Van der Linden and R. M. Boom, International dairy journal, 2008, 18, 1034-1042.

22. S. G. Bolder, H. Hendrickx, L. Sagis and E. Van der Linden, Applied rheology, 2006, 16, 258-264.

23. G. M. Kavanagh, A. H. Clark and S. B. Ross-Murphy, International Journal of Biological Macromolecules, 2000, 28, 41-50.

24. G. M. Kavanagh, A. H. Clark and S. B. Ross-Murphy, Langmuir, 2000, 16, 9584-9594.

25. W. S. Gosal, A. H. Clark and S. B. Ross-Murphy, Biomacromolecules, 2004, 5, 2420-2429.

26. D. Oboroceanu, L. Wang, E. Magner and M. A. Auty, Journal of Food Engineering, 2014, 121, 102-111. 
27. K. N. P. Humblet-Hua, G. Scheltens, E. van der Linden and L. M. C. Sagis, Food Hydrocolloids, 2011, 25, 569-576.

28. N. P. K. Humblet-Hua, E. van der Linden and L. M. C. Sagis, Journal of Agricultural and Food Chemistry, 2012, 60, 9502-9511.

29. T. B. J. Blijdenstein, C. Veerman and E. van der Linden, Langmuir, 2004, 20, 4881-4884.

30. J. Peng, A. Kroes-Nijboer, P. Venema and E. van der Linden, Soft Matter, 2016, 12, 3514-3526.

31. J. Peng, J. R. Simon, P. Venema and E. van der Linden, Langmuir, 2016, 32, 2164-2174.

32. J.-M. Jung and R. Mezzenga, Langmuir, 2009, 26, 504-514.

33. R. Mezzenga, J.-M. Jung and J. Adamcik, Langmuir, 2010, 26, 10401-10405.

34. F. Chiti and C. M. Dobson, Annu. Rev. Biochem., 2006, 75, 333-366.

35. C. M. Dobson, Nature, 2003, 426, 884-890.

36. C.-H. Tang and C.-S. Wang, Journal of Agricultural and Food Chemistry, 2010, 58, 11058-11066.

37. C. D. Munialo, A. H. Martin, E. van der Linden and H. H. de Jongh, Journal of Agricultural and Food Chemistry, 2014, 62, 2418-2427.

38. C. Akkermans, A. J. Van der Goot, P. Venema, H. Gruppen, J. M. Vereijken, E. Van der Linden and R. M. Boom, Journal of Agricultural and Food Chemistry, 2007, 55, 9877-9882.

39. C. Veerman, G. de Schiffart, L. M. C. Sagis and E. van der Linden, International Journal of Biological Macromolecules, 2003, 33, 121-127.

40. L. N. Arnaudov and R. de Vries, Biophysical Journal, 2005, 88, 515-526.

41. S. G. Bolder, L. M. C. Sagis, P. Venema and E. van der Linden, Journal of Agricultural and Food Chemistry, 2007, 55, 5661-5669.

42. S. Loveday, X. Wang, M. Rao, S. Anema, L. Creamer and H. Singh, International dairy journal, 2010, 20, 571-579.

43. S. Loveday, X. Wang, M. Rao, S. Anema and H. Singh, Journal of Agricultural and Food Chemistry, 2011, 59, 8467-8474.

44. C. Li and R. Mezzenga, Nanoscale, 2013, 5, 6207-6218.

45. C. Li and R. Mezzenga, Langmuir, 2012, 28, 10142-10146.

46. S. Bolisetty and R. Mezzenga, 2016, 11, 365-371.

47. E. Dickinson, Annual Review of Food Science and Technology, 2015, 6, 211-233.

48. E. van der Linden, Food Hydrocolloids, 2012, 26, 421-426.

49. I. Siró and D. Plackett, Cellulose, 2010, 17, 459-494.

50. R. J. Moon, A. Martini, J. Nairn, J. Simonsen and J. Youngblood, Chemical Society Reviews, 2011, 40, 3941-3994.

51. U. Geyer, T. Heinze, A. Stein, D. Klemm, S. Marsch, D. Schumann and H. P. Schmauder, International Journal of Biological Macromolecules, 1994, 16, 343-347.

52. R. Jonas and L. F. Farah, Polymer Degradation and Stability, 1998, 59, 101-106. 
53. J. M. Rajwade, K. M. Paknikar and J. V. Kumbhar, Applied Microbiology and Biotechnology, 2015, 99, 2491-2511.

54. L. Fu, J. Zhang and G. Yang, Carbohydrate Polymers, 2013, 92, 1432-1442.

55. Y. Huang, C. Zhu, J. Yang, Y. Nie, C. Chen and D. Sun, Cellulose, 2014, 21, 1-30.

56. N. Shah, M. Ul-Islam, W. A. Khattak and J. K. Park, Carbohydrate Polymers, 2013, 98, 1585-1598.

57. A. Nakagaito, S. Iwamoto and H. Yano, Applied Physics A, 2005, 80, 93-97.

58. N. Petersen and P. Gatenholm, Applied Microbiology and Biotechnology, 2011, 91, 1277-1286.

59. J. Shah and R. M. Brown Jr, Applied Microbiology and Biotechnology, 2005, 66, 352-355.

60. H. S. Barud, A. Tercjak, J. Gutierrez, W. R. Viali, E. S. Nunes, S. J. L. Ribeiro, M. Jafellici, M. Nalin and R. F. C. Marques, Journal of Applied Physics, 2015, 117, $17 \mathrm{~B} 734$.

61. N. Miyajima, K. Jinguji, T. Matsumura, T. Matsubara, H. Sakane, T. Akatsu and O. Tanaike, Journal of Physics and Chemistry of Solids, 2016, 91, 122-127.

62. M. Gama, P. Gatenholm and D. Klemm, Bacterial nanocellulose: a sophisticated multifunctional material, CRC Press, 2012.

63. A. M. A. Gallegos, S. H. Carrera, R. Parra, T. Keshavarz and H. M. Iqbal, BioResources, 2016, 11, 5641-5655.

64. A. F. Jozala, L. C. de Lencastre-Novaes, A. M. Lopes, V. de Carvalho Santos-Ebinuma, P. G. Mazzola, A. Pessoa-Jr, D. Grotto, M. Gerenutti and M. V. Chaud, Applied Microbiology and Biotechnology, 2016, 1-10.

65. S. M. Keshk, Journal of Bioprocessing \& Biotechniques, 2014, 4, 1.

66. E. Sukara and R. Meliawati, Jurnal Selulosa, 2014, 4, 7-16.

67. B. V. Mohite and S. V. Patil, Biotechnology and Applied Biochemistry, 2014, 61, 101-110.

68. M. Iguchi, S. Yamanaka and A. Budhiono, Journal of Materials Science, 35, 261-270.

69. F. Esa, S. M. Tasirin and N. A. Rahman, Agriculture and Agricultural Science Procedia, 2014, 2, 113-119.

70. Z. Shi, Y. Zhang, G. O. Phillips and G. Yang, Food Hydrocolloids, 2014, 35, 539-545.

71. A. Okiyama, M. Motoki and S. Yamanaka, Food Hydrocolloids, 1992, 6, 479-487.

72. A. Okiyama, M. Motoki and S. Yamanaka, Food Hydrocolloids, 1993, 6, 503-511.

73. A. Okiyama, M. Motoki and S. Yamanaka, Food Hydrocolloids, 1993, 6, 493-501.

74. S.-Y. Yu and K.-W. Lin, Journal of Food Science, 2014, 79, C1117-C1122.

75. P. Paximada, A. A. Koutinas, E. Scholten and I. G. Mandala, Food Hydrocolloids, 2016, 54, 245254.

76. K. W. Lin and H. Y. Lin, Journal of Food Science, 2004, 69, SNQ107-SNQ111.

77. S.-B. Lin, L.-C. Chen and H.-H. Chen, Journal of Food Process Engineering, 2011, 34, 1363-1379.

78. H. Ougiya, K. Watanabe, Y. Morinaga and F. Yoshinaga, Bioscience, Biotechnology, and Biochemistry, 1997, 61, 1541-1545. 
79. P. Paximada, E. Tsouko, N. Kopsahelis, A. A. Koutinas and I. Mandala, Food Hydrocolloids, 2016, 53, 225-232.

80. S. Lam, K. P. Velikov and O. D. Velev, Current Opinion in Colloid \& Interface Science, 2014, 19, 490-500.

81. M. Phisalaphong and N. Chiaoprakobkij, Bacterial Cellulose: A Sophisticated Multifunctional Material, 2012, 9, 143-156.

82. A. Kuijk, R. Koppert, P. Versluis, G. van Dalen, C. Remijn, J. Hazekamp, J. Nijsse and K. P. Velikov, Langmuir, 2013, 29, 14356-14360.

83. S. J. Veen, A. Kuijk, P. Versluis, H. Husken and K. P. Velikov, Langmuir, 2014, 30, 13362-13368.

84. S. J. Veen, P. Versluis, A. Kuijk and K. P. Velikov, Soft Matter, 2015, 11, 8907-8912.

85. D. de Kort, S. J. Veen, H. van As, D. Bonn, K. P. Velikov and J. van Duynhoven, Soft Matter, 2016, 12, 4739-4744.

86. H. N. W. Lekkerkerker and R. Tuinier, Colloids and the depletion interaction, Springer, Dordrecht ; New York, 2011.

87. G. A. Vliegenthart, A. van Blaaderen and H. N. W. Lekkerkerker, Faraday Discussions, 1999, 112, 173-181.

88. M. Adams, Z. Dogic, S. L. Keller and S. Fraden, Nature, 1998, 393, 349-352.

89. D. Guu, J. Dhont, G. Vliegenthart and M. Lettinga, Journal of Physics: Condensed Matter, 2012, 24, 464101.

90. Y. Mao, M. E. Cates and H. N. W. Lekkerkerker, Physical Review Letters, 1995, 75, 4548-4551.

91. K. H. Lin, J. C. Crocker, A. C. Zeri and A. G. Yodh, Physical Review Letters, 2001, 87.

92. G. A. Vliegenthart and H. N. W. Lekkerkerker, The Journal of Chemical Physics, 1999, 111, 41534157.

93. S. Asakura and F. Oosawa, Journal of Polymer Science, 1958, 33, 183-192.

94. S. Asakura and F. Oosawa, The Journal of Chemical Physics, 1954, 22, 1255-1256.

95. D. J. McClements, Food emulsions: principles, practices, and techniques, CRC press, 2015.

96. E. Dickinson, Food Hydrocolloids, 2003, 17, 25-39.

97. J.-L. Doublier, C. Garnier, D. Renard and C. Sanchez, Current Opinion in Colloid \& Interface Science, 2000, 5, 202-214.

98. V. Y. Grinberg and V. B. Tolstoguzov, Food Hydrocolloids, 1997, 11, 145-158.

99. V. Tolstoguzov, Food Hydrocolloids, 1991, 4, 429-468.

100. D. V. Zasypkin, E. E. Braudo and V. B. Tolstoguzov, Food Hydrocolloids, 1997, 11, 159-170.

101. L. Piculell, K. Bergfeldt and S. Nilsson, Biopolymer mixtures, 1995, 13-35.

102. I. T. Norton and W. J. Frith, Food Hydrocolloids, 2001, 15, 543-553.

103. E. Amici, A. H. Clark, V. Normand and N. B. Johnson, Biomacromolecules, 2000, 1, 721-729.

104. K.-Y. Lee, F. Quero, J. J. Blaker, C. A. S. Hill, S. J. Eichhorn and A. Bismarck, Cellulose, 2011, 18, 595-605. 
105. A. C. Dave, S. M. Loveday, S. G. Anema and H. Singh, International dairy journal, 2016, 57, 3943.

106. J.-M. Jung, D. Z. Gunes and R. Mezzenga, Langmuir, 2010, 26, 15366-15375.

107. P. A. Rühs, N. Scheuble, E. J. Windhab, R. Mezzenga and P. Fischer, Langmuir, 2012, 28, 1253612543.

108. P. A. Rühs, C. Affolter, E. J. Windhab and P. Fischer, Journal of Rheology (1978-present), 2013, 57, 1003-1022.

109. A. Kroes-Nijboer, H. Sawalha, P. Venema, A. Bot, E. Flöter, R. den Adel, W. G. Bouwman and E. van der Linden, Faraday Discussions, 2012, 158, 125-138.

110. R. I. Feigin and D. H. Napper, Journal of Colloid and Interface Science, 1980, 75, 525-541.

111. A. P. Gast and L. Leibler, The Journal of Physical Chemistry, 1985, 89, 3947-3949.

112. A. P. Gast and L. Leibler, Macromolecules, 1986, 19, 686-691.

113. G. Fleer and J. Scheutjens, Croatica chemica acta, 1987, 60, 477-494.

114. G. J. Fleer, J. H. M. H. Scheutjens and B. Vincent, in Polymer Adsorption and Dispersion Stability, American Chemical Society, 1984, vol. 240, pp. 245-263.

115. B. Vincent, J. Edwards, S. Emmett and A. Jones, Colloids and Surfaces, 1986, 18, $261-281$.

116. B. Vincent, P. F. Luckham and F. A. Waite, Journal of Colloid and Interface Science, 1980, 73, 508-521.

117. Y. Mao, M. E. Cates and H. N. W. Lekkerkerker, Physica A: Statistical Mechanics and its Applications, 1995, 222, 10-24.

118. S. Kim, K. Hyun, J. Y. Moon, C. Clasen and K. H. Ahn, Langmuir, 2015, 31, 1892-1900.

119. J. Y. Walz and A. Sharma, Journal of Colloid and Interface Science, 1994, 168, 485-496.

120. A. L. Ogden and J. A. Lewis, Langmuir, 1996, 12, 3413-3424.

121. P. G. De Gennes, Macromolecules, 1976, 9, 587-593.

122. F. C. MacKintosh, auml, J. s and P. A. Janmey, Physical Review Letters, 1995, 75, 4425.

123. C. F. Schmidt, M. Baermann, G. Isenberg and E. Sackmann, Macromolecules, 1989, 22, 36383649.

124. L. Onsager, Annals of the New York Academy of Sciences, 1949, 51, 627-659.

125. A. Stroobants, H. N. W. Lekkerkerker and T. Odijk, Macromolecules, 1986, 19, 2232-2238.

126. A. R. Khokhlov and A. N. Semenov, Physica A: Statistical Mechanics and its Applications, 1981, 108, 546-556.

127. G. J. Vroege and H. N. W. Lekkerkerker, Reports on Progress in Physics, 1992, 55, 1241-1309.

128. G. J. Vroege and H. N. Lekkerkerker, Reports on Progress in Physics, 1992, 55, 1241.

129. D. Lin, R. Li, P. Lopez-Sanchez and Z. Li, Food Hydrocolloids, 2015, 44, 435-442.

130. E. Dickinson and L. Eriksson, Advances in Colloid and Interface Science, 1991, 34, 1-29.

131. D. H. Napper, Journal of Colloid and Interface Science, 1977, 58, 390-407. 
132. G. Agoda-Tandjawa, S. Durand, S. Berot, C. Blassel, C. Gaillard, C. Garnier and J. L. Doublier, Carbohydrate Polymers, 2010, 80, 677-686.

133. M. P. Lowys, J. Desbrières and M. Rinaudo, Food Hydrocolloids, 2001, 15, 25-32.

134. M. Pääkkö, M. Ankerfors, H. Kosonen, A. Nykänen, S. Ahola, M. Österberg, J. Ruokolainen, J. Laine, P. T. Larsson, O. Ikkala and T. Lindström, Biomacromolecules, 2007, 8, 1934-1941.

135. S. Bolisetty, L. Harnau, J.-m. Jung and R. Mezzenga, Biomacromolecules, 2012, 13, 3241-3252.

136. C. Veerman, H. Baptist, L. M. C. Sagis and E. van der Linden, Journal of Agricultural and Food Chemistry, 2003, 51, 3880-3885.

137. J.-M. Jung, G. Savin, M. Pouzot, C. Schmitt and R. Mezzenga, Biomacromolecules, 2008, 9, 2477-2486.

138. S. Jordens, P. A. Rühs, C. Sieber, L. Isa, P. Fischer and R. Mezzenga, Langmuir, 2014, 30, 1009010097.

139. D. Pimentel and M. Pimentel, The American journal of clinical nutrition, 2003, 78, 660S-663S.

140. J. Sabaté and S. Soret, The American journal of clinical nutrition, 2014, 100, 476S-482S.

141. X. Zhang, M. R. Servos and J. Liu, Journal of the American Chemical Society, 2012, 134, 99109913. 
Chapter 9 


\section{Summary}


Fibrillar structures are important structuring elements for food products. Understanding the behaviour of fibrillar structures in complex food systems is essential for successful industrial applications. This thesis presents the behaviour of two different fibrillar structures, i.e. whey protein isolate (WPI) fibrils and bacterial cellulose (BC) microfibrils in mixtures under various conditions. The WPI fibrils are prepared from WPI by heating a WPI solution at $\mathrm{pH} 2$ at $80{ }^{\circ} \mathrm{C}$ for several hours. The $\mathrm{BC}$ microfibrils are extracted from commercial available 'Nata de Coco' by high-energy de-agglomeration. In Chapter 1, a general introduction is given, where we introduce two different fibrillar structures that were studied in this thesis. Also, the aim and the outline of the thesis are presented.

In Chapter 2, we studied the stability, rheology and microstructure of the mixed dispersions containing WPI and $\mathrm{BC}$ microfibrils at $\mathrm{pH} 7$ both before and after heating. We focused on WPI concentrations that are below the critical gelling concentration; thus, the mixtures studied are all in the liquid state. Results showed that the mixtures form stable dispersions at BC microfibril concentrations larger than $0.2 \mathrm{wt} \%$. The rheological properties of the mixtures are dominated by the presence of $B C$ microfibrils. The mixtures are thixotropic and flow curves show two shear thinning regions and a viscosity plateau in between these regions. Upon reversal of the shear rate, the viscosity decreased, i.e. the mixtures show hysteresis. After heating at $80^{\circ} \mathrm{C}$ for $30 \mathrm{~min}$, the pure $\mathrm{BC}$ dispersions did not show any difference in stability, rheological properties and microstructure. The mixtures had the same stability as before heating. BC is still the main contributor to the viscosity of the mixture. The viscosity plateau and hysteresis were still observed in the mixtures containing up to 6 wt\% WPI, but were much less pronounced compared to the mixtures containing 9 wt\% WPI. The presence of BC microfibrils in the WPI solutions did not influence the denaturation temperature of the WPI during heating. The WPI aggregates formed during heating were not influenced by the presence of $B C$ microfibrils and vice versa. The viscosity of the mixtures did not differ significantly when mixing $B C$ microfibrils into WPI before or after heating the WPI solution. We conclude that the WPI and $B C$ microfibrils did not show specific interactions when mixed at $\mathrm{pH} 7$. The denaturation and aggregation of WPI during heating was not influenced by the presence of $\mathrm{BC}$ microfibrils. In Chapter 3, we investigated the stability, rheology and microstructure of mixtures at $\mathrm{pH} 2$ containing WPI fibrils and $\mathrm{BC}$ microfibrils. At $\mathrm{pH} 2$, the WPI is positively charged and forms fibrils when heated for 22 hours at $80{ }^{\circ} \mathrm{C}$. The mixtures remained stable for 7 days at BC concentrations larger than $0.2 \mathrm{wt} \%$. The flow curves of 
the mixtures are comparable to those of the pure $B C$ dispersions at the same $B C$ microfibril concentration. Similar to the results in Chapter 2, two shear-thinning regions and a viscosity plateau in between them are observed. The mixtures are thixotropic and show hysteresis, i.e. lower viscosity is measured when shear rate is reversed. After prolonged heating at $80^{\circ} \mathrm{C}$, the macroscopic stability of the mixtures remained unchanged for 7 days. The pure BC dispersion did not show much difference on stability, viscosity and microstructure in comparison to the $\mathrm{BC}$ dispersion before heating. Interestingly, the viscosity plateau and the hysteresis upon the reversal of shear rate were absent in the flow curves of the mixtures after heating. No significant difference was observed in viscosity when BC microfibrils were mixed with the WPI solution either before or after heating the WPI solution. The microstructure of BC microfibrils was not influenced when heated in the presence of WPI. The structure of the mixtures consists of two independent networks. The presence of BC microfibrils leads to shorter WPI fibrils, as was qualitatively observed from microscopy images.

By increasing the WPI concentration above the critical gelling concentration in the WPI-BC mixtures at $\mathrm{pH} 7$, heat-induced WPI-BC gels at different $\mathrm{NaCl}$ concentrations were prepared. In Chapter 4, we characterized the rheology, mechanical properties, microstructure and water holding capacity of these gels. The presence of BC microfibrils did not affect the gelation dynamics of WPI, although the elastic modulus $\mathrm{G}^{\prime}$ of the gel is increased with increasing $B C$ concentration. This increase is not observable when the strength of the WPI gel is high enough. In this case the rheology of the WPI-BC gel is dominated by the WPI. The microstructure of the WPI-BC gel can be seen as a duplex gel that consists of two independent networks. With increasing $\mathrm{NaCl}$ concentration, the $\mathrm{BC}$ gel remains unchanged, while WPI gel changes from fine stranded to particulate structure. The WPI gel structure in the duplex gels is the same as the structure of the WPI gels that are formed in the absence of $\mathrm{BC}$ microfibrils, regardless of $\mathrm{BC}$ and $\mathrm{NaCl}$ concentration. The mechanical properties (i.e. large deformation rheology) and water holding capacity of the duplex gels were controlled mainly by the properties of the WPI gel. We conclude that the heat-induced gelation of WPI in the WPI-BC mixtures is the same as when heated in the absence of BC microfibrils.

In Chapter 5, gels of WPI-BC mixtures were prepared at pH 2 after heating at $80{ }^{\circ} \mathrm{C}$ for 10 hours. The presence of $B C$ microfibrils did not influence the formation of the WPI fibrillar gel. Increasing the BC concentration in the WPI-BC mixtures resulted in a more brittle gel. 
Interestingly, the storage modulus and loss modulus have the same dependency on BC concentration. From the microstructure we conclude that a bi-fibrillar gel is formed, consisting of two mutually independent networks, i.e. a WPI fibrillar and BC microfibrillar network. The WPI fibrils formed in these bi-fibrillar gels seemed to be more aligned in comparison to the fibrils formed in the absence of $B C$ microfibrils. The alignment of the fibrils increases, when they are closer to the $B C$ microfibrils or when the $B C$ concentration increases. The large deformation properties of the bi-fibrillar gels are dominated by the WPI fibrillar network and, in turn, the properties of fibrils building this network are controlled by the $\mathrm{NaCl}$ concentration.

The stability of a WPI-stabilized oil-in-water (o/w) emulsion in the presence of WPI fibrils is discussed in Chapter 6. With increasing fibril concentration, the emulsions were subject to creaming, enhanced creaming and stabilization, respectively. The enhanced creaming observed above a certain fibril concentration is caused by depletion flocculation, as induced by the fibrils. The microstructure of the stabilized emulsion at higher fibril concentration reveals the presence of both single droplets and aggregates of oil droplets. Interestingly, the aggregates reach a finite size that is independent of the fibril concentration. Upon dilution by either the solvent or the fibril solution at the same concentration, the aggregation was found to be reversible. The viscosity of the stabilized emulsions was dominated by the viscosity of fibrils. No gelation of fibrils and/or oil droplets was observed. Diffusing wave spectroscopy (DWS) shows that the oil droplets and their aggregates are still relatively free to diffuse over short length scales, while they are trapped at larger length scales. We speculated that the aggregation of oil droplets in the stabilized emulsions is still caused by depletion interaction. However, at high enough fibrils concentrations, the network formed by the fibrils has a small mesh size, which prevents that larger aggregates can easily approach each other. We speculate that the stabilization mechanism finds its origin in the presence of a kinetic barrier. Furthermore, we observed that the emulsions stabilized by fibrils remain their stability at higher temperature and the longer fibrils are more effective in stabilizing the emulsion.

In Chapter 7, we studied the behaviour of WPI fibrils in a colloidal dispersion containing monodispersed polystyrene latex particles at $\mathrm{pH}$ 2. At this $\mathrm{pH}$ the fibrils and latex particles are oppositely charged. Latex particles with two different sizes were used. As a function of fibril concentration, the system showed four different stability regimes: 1) bridging flocculation, 2) steric and/or electrostatic stabilization, 3) depletion flocculation and 4) 
depletion stabilization. The bridging flocculation was caused by the adsorption of fibrils on the oppositely charged latex particles. When the surface of the particles is fully covered by the fibrils, the system reaches steric and/or electrostatic stabilization. Further addition of fibrils cause depletion flocculation as induced by the non-adsorbed fibrils. At even higher fibril concentration, the emulsions showed stabilization with features similar to the stabilization in emulsions by fibrils presented in Chapter 6 . When smaller latex particles are present, the onset of stabilization and de-stabilization takes place at higher fibril concentrations than in case of the larger particles. When the surface of the latex particles is modified by the adsorption of lysozyme the bridging flocculation did not occur as the lysozyme-coated particles are now also positively charged like the fibrils. The depletion flocculation and depletion stabilization at higher fibril concentration still occurred. In all dispersions stabilized by the fibrils, we observed both single latex particles and small clusters of particles present together, where the small clusters do not grow beyond a certain size. We proposed a stabilization mechanism similar to the mechanism in emulsions stabilized by fibrils, i.e. depletion stabilization due to the presence of a kinetic barrier. Additionally, diffusing wave microscopy was found to be an effective technique to monitor the kinetics of particle aggregation.

In Chapter 8 the stability of emulsions at $\mathrm{pH} 2$ in the presence of only $\mathrm{BC}$ microfibrils and in the presence of both BC microfibrils and WPI fibrils was studied. When only BC microfibrils added at a sufficiently high concentration, the emulsions are stabilized by the presence of a yield stress as generated by the BC network. When both WPI fibrils and BC microfibrils are added to the emulsions, the networks they form behave in the same way, as when they are added to the emulsions separately. The WPI fibrils induced depletion flocculation and stabilization of the emulsions, despite the presence of the $\mathrm{BC}$ microfibrils. However, at high enough BC microfibril concentrations, the emulsions can be stabilized against depletion flocculation as induced by the WPI fibrils. The competition between stabilization and/or de-stabilization induced by the BC microfibrils and the WPI fibrils can lead to emulsions with different microstructures and rheological properties. In addition, the stability of emulsions induced by both BC microfibrils, and bi-fibrillar networks from mixtures of WPI fibrils and BC microfibrils, is temperature independent.

A general discussion on the results obtained in this thesis is presented in Chapter 9, which includes recommendations for further research and concluding remarks. 


\section{Acknowledgements}

It took me a while to finally sit down and write this part. It was an emotional moment when I wrote down "acknowledgements". I meant 'I feel like crying', for someone that doesn't cry easily. I was thinking how I am going to express my deep gratitude with several pages of words to many people who have helped me to reach this point. When I started my PhD, I thought 4 years is a long period; now I think it went so fast. I have been a very lucky person during my PhD journey, thanks to my great supervisors, colleagues and friends, who have made these 4 years definitely happy and memorable.

My most sincere gratitude goes to my supervisors. I would not have been able to achieve this without their significant contribution and support. Erik van der Linden has always been very supportive and positive during my PhD. He has inspired me from many different perspectives and motivated me all the time with his very positive feedback. He has been always very patient and generous to help me and to listen to me, not only to give me guidance for my work, but also many advices for my personal development. I have learnt to be very precise and critical in scientific research thanks to his constructive feedback and continuous encouragement. I have enjoyed our meetings and chats very much and I felt very comfortable to express myself freely, no matter the topic of discussion. I really appreciate his support during the last busy period of my PhD, especially to help me trust myself on achieving this goal. It has been a real pleasure and fortunate to have him as my supervisor. I also owe many thanks to Paul Venema, who has given me many insightful ideas and suggestions for my research. He has given me a lot of freedom and opportunities to grow and explore many ideas in the lab. His curiosity and interest in different things has also inspired me in many aspects. We had plenty coffee breaks to discuss science and other interesting matters with a lot of fun and laughter. Whenever I needed help, he was always there, no matter how I did with my research, he was always there to back me up. Thanks to his support and trust, I felt confident about my work and free to talk anything with him, even if it was a silly question. Apart from work, we have also shared many nice stories and funny jokes, which resulted from a combination of science, research and fun during our meetings with Erik van der Linden. It was impossible 
for me to feel bored at these meetings. I consider him as a wise person, and as my supervisor, it was a great pleasure to work with him for both science and fun. I am also thankful to Krassimir P. Velikov, who has provided me with insightful discussions and ideas. He is critical yet also supportive. Special thanks to him for providing the cellulose for my experiments and lab support at Unilever, which helped my research run smoothly.

My great gratitude also goes to my thesis committee members, Prof. Dr J.P.M. van Duynhoven, Prof. Dr J. van der Gucht, Prof. Dr A.J. van der Goot and Dr R.H. Tromp. I am indebted to them for reading and evaluating my thesis as well as coming to my defence.

My project is part of a NanoNextNL project. Thus, my sincere gratitude also goes to the NanoNextNL program office: Freya Senf, Sylvia van Mildert, Titia Plantinga, Sharda Parmanand, Rens Vandeberg, Melvin Kasanrokijat and many others. Thanks to them all for organizing many great courses, meetings and conferences, at which I met a lot of people, had many fruitful discussions and learnt a lot.

I would also like to thank Sandra J. Veen and Anke Kuijk for their lab support in cellulose prepparation, rheology measurements and TEM analysis, as well as the insightful discussions we had. I am also grateful to Peter Versluis for his help on the rheology measurements. In addition, my sincere gratitude goes to Daan de Kort for helping me with the Rheo-NMR measurements; and to John van Duynhoven and Henk van As for their input on the discussions of Rheo-NMR. I am also thankful to Tiny Franssen-Verheijen for SEM analysis and Norbert de Ruijter for CLSM instructions.

I have been very happy and fortunate to do my PhD in the Food Physics group, where I have had absolutely a wonderful time during the last 4 years. Although the Dutch weather was often terrible, working in the Food Physics group gave me enough sunshine, thanks to the great atmosphere created by my lovely colleagues. I am very indebted to Els Jansen, who has helped me not only with all the administrative work to make my PhD life much easier, but also with all kinds of things, like finding a perfect bike for me. She is truly an amazing and caring person, as I always said; she is like a mom, cared about everyone and everything. Elke Sholten is another person that I am very grateful to. I have received many great advices and help from her during my PhD. Every time I went to her office, she was always very patient to listen to me and willing to give me a hand. She is critical yet very easy-going and an open-minded person; that made me feel free to share what I think with her. Our drinks, parties and dinners brought a lot of fun to my life after work. I would 
always miss the moment that she came to my office and called me "pingping" with a cheerful voice and a big smile on her face to ask how I was doing and to say goodbye after a long day. This always made me happy.

My special thanks also go to Harry Baptist and Miranda de Beus for their effort to make sure everything runs smoothly in the lab. Without their help, my experiments would not have finished always on time. They always had a solution to all my problems. I am also very grateful to their patience for helping me with the TEM analysis (Harry) and CLSM analysis (Miranda), which sometimes took very long time. I also want to thank Leonard Sagis for the discussions we had on all kinds of physics questions, I have been impressed by his great way of explaining science. I am also indebted to my dear friend Anika Oppermann, who has initiated and organized many great activities for us during my PhD. We had a lot of great time and beautiful memories together. I am grateful to her patience and kindness to help me and listen to me. She has cared about me a lot, cheered me up and motivated me all the time, especially in the last busy period of my PhD. She helped me keeping motivated, either by bringing nice food or writing me a lovely card. I have learnt a lot from her and benefited a lot from her kindness and great ideas. I am also very grateful to have her as a paranymph by my side. My special gratitude goes to Kun Liu, my very close friend during my PhD, with whom I shared and learnt all kinds of things. She is special and unique. She was always there and helped me whenever I need it. She was also great at cheering me up and encouraging me, especially to be confident with myself. She pays attention to what I like and what I need and would always surprise me in different ways. I cannot list the plenty times she had helped me, but I thankful for all the wonderful time together. She is like my old sister that I can always trust. There is also my special friend Vaida Urbonaite, being my colleague during my PhD and also my current job. I am very happy to have her being close to me, so that we can hang out when we have time. She is a great person to talk to, always understand what I think and what I say. I have received many great ideas and advices from her, both for work and for personal life. She has helped me in many different things, and I could not tell clearly how much I have benefited from her help. I am also grateful to Carsten Ersch, who has been very generous to share his knowledge with me and to greatly support me in the lab and to advise me during my PhD. He has been always willing to help as much as he can. I also want to give my thanks to Auke de Vries for all his great jokes, from which I laughed a lot and had so much fun. Anja Oechsle, I am very happy and grateful that you came to food physics 
group in my last year. I have enjoyed so much chatting with you and hanging out with you. You are such a cheerful and caring person, I have learnt a lot from you. Thanks to you and Anika, I have also learnt to ski. I also want to thank my other colleagues: Guido, Pauline, Alev, Jacob, Nam-Phuong, Jissy, Zhili, Carol, Claire, Tijs, Philipp, May, Arianne, Belinda, Claudine, Marco, Monica, Leen, Lenka, Laura, Maria, Dilek, Elisabeth for all their help, support, fun and laughter both at work and outside work. All of them have helped me in many different ways and different things which absolutely meant a lot to me.

During my PhD, I have supervised 7 students for their thesis: Koen, William, Joana, Julia, Nina, Ruben and Vincenzo. I want to specially thank them all for their hard-working, curiosity, great ideas and initiatives. Apart from their contribution to the thesis, I have also learnt a lot from them and enjoyed very much working with them. I am very happy and proud for the work we have done together. It was absolutely a great experience.

I am also very grateful to my friends outside work, with whom I had a lot of great time and memories together. Thanks to them, my spare time has always been filled with a lot of nice activities and I never felt alone. My special and closest friend since my Master study, Angelica Tamayo, also one of my paranymphs, I cannot tell how lucky and grateful I felt to have her around all the time. Almost every week, we have managed to meet at least once for lunch or dinner (I am very proud of that). It has always been a joyful and relaxing moment, and it was something that I have always looked forward to. We have spent a lot of great time and shared many things together, our ups and downs, good and bad news, complaints and problems etc. Whenever there is something, she is one the first ones I would go and talk to. I have learnt and benefited a lot from her patience in listening to me and her countless great advices. I was super happy that she came together with her husband Daniel to visit my family in China during the New Year in 2015, where we had a wonderful time together.

Special thanks to Fahui Liu for helping me design the thesis cover, although he had a busy schedule. I am also very thankful to my friends: Xiaoxiang, Junyou, Junli, Wenjuan, Xuezhen, Liya, Fahui, Lina, Chunyue, Ya, Fangjie, Yuxi, Lingmin, Red, Lu, Qi, Jun, Zhaojun, Yongfu, Juan, Jue, Feifei, Tian, Na, Jingyan, Siew Ling, LooWee, Geraldine, Marine, Sara, Filipos, Sami, Grace, Paola, Fred, Pierre, Akemi. I enjoyed very much all the nice parties, dinners, chats and all kinds of other activities we had together. 
I would not forget to give my deepest gratitude to my dearest parents and my brother. I owe many thanks to them, for their unconditional love, support and tolerance to my bad temper all the time. My dearest parents, they have always been doing their best to give me the best. They give me the freedom to do whatever I like. My dearest brother, although I am 2 years older than him, in fact, he has behaved more like an older brother, caring about me and bearing my bad temper. Thanks to them, I have never felt alone whenever and wherever I go. Thanks to them, I can feel free to pursue my dreams and to do the things I like. 感谢我最亲爱的爸爸妈妈还有我的弟弟, 感谢你们无私的爱和支 持. 你们教会我善良与真诚, 包容和理解, 懂得感恩和有责任心，太多太多. 感谢你 们一直包容我的坏脾气和任性。你们总是把最好的给我, 总是努力给我创造一个自由 和美好的环境, 让我安心学习和工作,不要担心任何事情。也感谢我的弟弟, 虽然我 是姐姐, 但你总是比我更懂事, 包容我的坏脾气, 体贴我的难处, 照顾我, 关心我, 帮助我. 因为有你们, 我才可以那么无所畏惧的去追求自己的梦想.

There is another important person I cannot forget to thank; it is my boyfriend, Gongbao. Without him I probably could not have finished my thesis on time. The thanks I owe him are more than words can say. He has done a lot for me, from unconditionally loving and supporting me to tolerating all kinds of temper, which often changed as frequent as the Dutch weather. Whenever and wherever I need help, he is always there, full of patience to listen to me, understand me and find a solution or cheer me up. In the last year of my PhD, he has travelled every weekend from Groningen to do grocery shopping, cleaning and cooking for me. Thanks to his support, I can fully concentrate on finishing my thesis. I feel really lucky, proud and happy to be with him.

Jinfeng 金峰

Pingping 萍萍 


\section{About the author}

Jinfeng Peng was born on October 21, 1988 in Ganzhou, Jiangxi Province, China. After completing her secondary education in 2006, Jinfeng started her Bachelor study in Food Science and Engineering at Hainan University, Haikou, China. In 2010 she got her Bachelor degree and obtained the scholarship to study her Master in Wageningen University, the Netherlands. She studied Food Technology with the specialization of European Master in Food Studies. During the first year of her MSc, she studied in 4 different countries, traveling from the Netherlands, Ireland, and France to Sweden. In September 2011, Jinfeng got the opportunity to do her Master thesis and internship

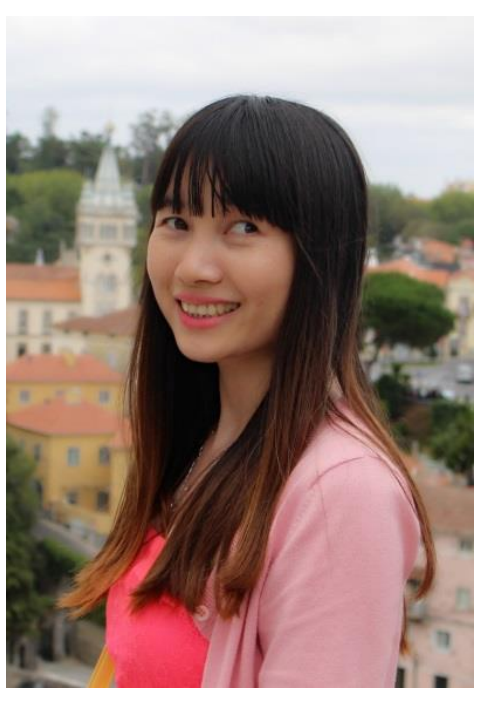
together in Unilever R\&D Vlaardingen, the Netherlands. During the MSc study, Jinfeng has also worked with a team of 11 international students on a two-year big project, aiming to develop meat patties from insects. In September 2012, Jinfeng started as a PhD researcher in the Laboratory of Physics and Physical Chemistry of Foods, Wageningen University. Her research was part of the project "Molecular structure of Food" sponsored by NanoNextNL. The results of her PhD research are presented in this thesis. Currently Jinfeng is working as a scientist in the Technology Department of Advanced Medical Nutrition at Danone Nutricia Research, Utrecht, the Netherlands.

She can be reached by email: pengif1988@gmail.com 


\section{List of publications}

\section{Peer reviewed publications}

Jinfeng Peng, Joana Ralfas Simon, Paul Venema, Erik van der Linden. Protein fibrils induce emulsion stabilization. Langmuir, 2016, 32, 2164-2174.

Jinfeng Peng, Ardy Kroes-Nijboer, Paul Venema, Erik van der Linden. Stability of colloidal dispersions in the presence of protein fibrils. Soft Matter, 2016, 12, 3514-3526.

Jinfeng Peng, Vincenzo Calabrese, Sandra J. Veen, Peter Versluis, Krassimir P. Velikov, Paul Venema, Erik van der Linden. Rheology and microstructure of dispersions of whey proteins and cellulose microfibrils. (to be submitted)

Jinfeng Peng, Vincenzo Calabrese, Sandra J. Veen, Peter Versluis, Krassimir P. Velikov, Paul Venema, Erik van der Linden. Rheology and microstructure of dispersions of protein fibrils and cellulose microfibrils. (to be submitted)

Jinfeng Peng, Vincenzo Calabrese, William Nicholas Ainis, Ruben Scager, Krassimir P. Velikov, Paul Venema, Erik van der Linden. Duplex gels from whey protein isolate and cellulose microfibrils. (to be submitted)

Jinfeng Peng, Vincenzo Calabrese, Julia Geurtz, Krassimir P. Velikov, Paul Venema, Erik van der Linden. Directed growth of protein fibrils in the presence of cellulose microfibrils. (to be submitted)

Jinfeng Peng, Nina van der Hulst, Krassimir P. Velikov, Paul Venema and Erik van der Linden. Tuning emulsion stability using protein-cellulose bi-fibrillar networks. (to be submitted)

Jinfeng Peng, Vincenzo Calabrese, Krassimir P. Velikov, Paul Venema, Erik van der Linden. Effect of high pressure on dispersions of whey proteins and cellulose microfibrils. (to be submitted)

Jinfeng Peng, Krassimir P. Velikov, Paul Venema, Erik van der Linden. Behaviour of Fibrillar structures in complex systems, a review. (to be submitted) 


\section{Patents}

Arjen Bot, Jinfeng Peng, Qingguo $\mathrm{Xu}$, and Shiping Zhu. AERATED OIL CONTAINING SUCROSE FATTY ACID ESTER AND HYDROPHOBIN." U.S. Patent No. 20,150,086,701. 26 Mar. 2015.

Deborah Lynne Aldred, Arjen Bot, Penelope Eileen Knight, Jinfeng Peng, Jan Alders Wieringa, Qingguo $\mathrm{Xu}$, and Shiping Zhu. METHOD FOR PRODUCTION OF AERATED WATERIN-OIL EMULSIONS AND AERATED EMULSIONS. U.S. Patent No. 20,150,327,565. 19 Nov. 2015. 


\section{Overview of completed training activities}

\section{Discipline specific activities}

2012, Emulsions and Foams Conference, Lunteren, NL

2013, $14^{\text {th }}$ European School on Rheology, Leuven, BE

2013, $14^{\text {th }}$ European Student Colloid conference, Potsdam-Golm, DE

2013, $3^{\text {rd }}$ EPNOE International Polysaccharide Conference, Nice, FR

2013, EPNOE pre-conference course, Nice, FR

2013, Biopolymers 2013, Nantes, FR

2013, MicroNano Conference '13, Ede, NL

2014, $15^{\text {th }}$ Food Colloids Conference, Karlsruhe, DE ${ }^{\text {a }}$

2014, UK Colloids 2014, London, UK ${ }^{\text {b }}$

2014, Nanocity 2014, Utrecht, NL ${ }^{b}$

2015, Cellulose Technology Course, Turku, FI

$2015,7^{\text {th }}$ International Symposium on Food Rheology \& Structure, Zurich, SZ ${ }^{\text {b }}$

$2015,6^{\text {th }}$ International Symposium on Delivery of Functionality, Paris, FR ${ }^{\text {b }}$

2015, Nanocity 2015, Amersfoort, NL ${ }^{\text {b }}$

2015, EPNOE pre-conference course, Warsaw, $\mathrm{PL}^{\mathrm{b}}$

2015, $4^{\text {th }}$ EPNOE International Polysaccharide Conference, Warsaw, PL ${ }^{a}$

2015, NanoNextNL Food Theme meeting, Wageningen, NL ${ }^{b}$

2016, $16^{\text {th }}$ Food Colloids Conference, Wageningen, $\mathrm{NL}^{\mathrm{a}}$

2016, Nanocity 2016, Amsterdam, NL ${ }^{a+b}$

\footnotetext{
${ }^{*}$ a talk, ${ }^{b}$ poster
} 


\section{General courses}

2012, VLAG PhD week, Baarlo, NL

2013, PhD competence assessment, Wageningen, NL

2013, Project and Time Management, Wageningen, NL

2013, Techniques for Writing and Presenting a Scientific Paper, Wageningen, NL

2013, NanoNextNL Risk Analysis and Technology Assessment (RATA) course, Arnhem, NL

2013, NanoNextNL IP Awareness and Valorisation course, Amersfoort, NL

2014, Data management, Wageningen, NL

2014, NanoNextNL Entrepreneurship course, Soesterborg, NL

2014, Information Literacy for PhD including EndNote Introduction, Wageningen, NL

2014, Efficient writing strategies, Wageningen, NL

2014, Scientific publishing workshop, Wageningen, NL

2014, Presentation skills, Wageningen, NL

2014, NanoNextNL Analytic storytelling course, Utrecht, NL

2015, Mobilising your scientific network, Wageningen, NL

2015, Brain training, Wageningen, NL

2015, Communication with the media and the general public, Wageningen, NL

2015, Interpersonal communication for PhD candidates, Wageningen, NL

2015, Career perspectives, Wageningen, NL

\section{Optional activities}

2012, Preparation of research proposal, Wageningen, NL

2012, Feedback course, Wageningen, NL

2014, Participation and organization of PhD study tour to USA and Canada

2012-2016, Weekly group meetings, FPH, Wageningen, NL

2012-2016, NanoNextNL Project meetings, NanoNextNL, Utrecht, NL 


\section{Colophon}

This thesis is part of NanoNextNL, a micro and nanotechnology innovation consortium of the Government of the Netherlands and 130 partners from academia and industry.

Financial support from Wageningen University for printing this thesis is gratefully acknowledged.

Cover Design: Fahui Liu

Layout: Jinfeng Peng

Printed by GVO drukkers \& vormgevers B.V. 